\title{
Modelling Railway Bridge Asset Management
}

\author{
Bryant Linh Hai Le
}

A Doctoral Thesis

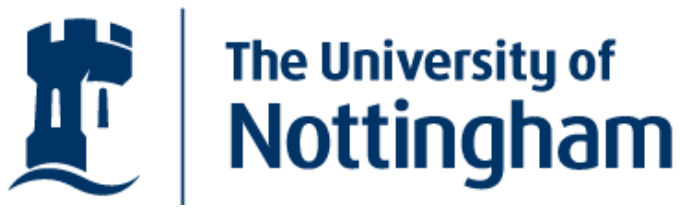

Thesis submitted to the University of Nottingham for the degree of Doctor of Philosophy

January 2014 




\section{Abstract}

The UK has a long history in the railway industry with a large number of railway assets. Railway bridges form one of the major asset groups with more than 35,000 bridges. The majority of the bridge population are old being constructed over 100 years ago. Many of the bridges were not designed to meet the current network demand. With an expected increasing rate of deterioration due to the increasing traffic loads and intensities, the management authorities are faced with the difficult task of keeping the bridge in an acceptable condition with the constraint budget and minimum service disruptions. Modelling tools with higher complexity are required to model the degradation of assets and the effects of different maintenance strategies, in order to support the management decision making process.

This research aims to address the deficiencies of the current bridge condition systems and bridge models reported in the literature and to demonstrate a complete modelling approach to bridge asset management. The degradation process of a bridge element is studied using the historical maintenance data where previous maintenance actions were triggered by a certain type of defects. Two bridge models are then developed accounting for the degradation distributions, service and inspection frequency, repair delay time and different repair strategies. The models provide a mean of predicting the asset future condition as well as investigating the effects of different maintenance strategies will have on a particular asset. The first model is a continuous-time Markov bridge model and is considered more complex than other models in the literature, the model demonstrates the advantages of the Markov modelling technique as well as highlighting its limitations. The second bridge model presented a novel Petri-Net modelling approach to bridge asset modelling. This stochastic modelling technique allows much more detail modelling of bridge components, considering: non-constant deterioration rates; protective coating modelling; limits of the number of repairs can be carried out; and the flexibility of the model allows easily extension to the model or the number of components modelled. By applying the two models on the same asset, a comparison can be made and the results further confirm the validations and 
improvements of the presented Petri-Net approach. Finally, optimisation technique (Genetic Algorithm) is applied to the bridge models to find the optimum maintenance strategies in which the objectives are to minimise the whole life cycle cost whist maximising the asset average condition. A hybrid optimisation that takes advantage of both bridge models, resulting in a significant time saving, is also presented. 


\section{Acknowledgements}

First and foremost, I would like to express my sincere appreciation to Professor John Andrews, for the continuous support throughout my PhD research, for his patience, motivation and immense knowledge. His guidance has helped me tremendously in all the time of researching and writing this thesis. I am extremely grateful to have him as my supervisor and believe that he is the best supervisor one could have. Special thanks to Dr Rasa Remenyte-Prescott and Dr Daren Prescott for their help and advice over the last few years.

I would also like to thank Andy Kirwan for the sponsorship, and giving me the opportunity to start on this project. His willingness to support this project is very much appreciated. I wish to thank Sam Chew and Julian Williams and other members of Network Rail for their valuable inputs and knowledge throughout the development of this research.

I would like to extend my thanks to other member of Infrastructure Asset Management group and the people of the Nottingham Transportation Engineering Centre for their assistance, encouragement and friendship. I am very fortunate to have met all these lovely people. Thanks to Matthew Audley who has always made my time in the office more fun. Thanks to all other people in the group who have played cards with me at lunch time and have made my time more enjoyable.

Finally, I wish to thank my mom, my dad, my brother and his family, and all my friends for their constant support and understanding throughout my research. Without them this thesis would not have been possible. 


\section{Publications}

\section{Journal articles}

LE, B. \& ANDREWS, J. 2013. Modelling railway bridge asset management. Proceedings of the Institution of Mechanical Engineers, Part F: Journal of Rail and Rapid Transit, 227, 644656.

\section{Conference papers}

LE, B. \& ANDREWS, J. 2012. Railway bridge asset management modelling. In Proceedings of Rail Research UK Association Annual (RRUKA) Conference 2012, $7^{\text {th }}$ November, London 2012.

LE, B. \& ANDREWS, J. 2013. A Markov modelling approach to railway bridge asset management. In Proceedings of AR2TS seminar: advances in risk and reliability technology symposium, Loughborough, United Kingdom, 2013. 


\section{Abbreviations}

$\begin{array}{ll}\text { ABT } & \text { Abutment } \\ \text { BGL } & \text { Bearing } \\ \text { CAF } & \text { Cost Analysis Framework } \\ \text { CARRS } & \text { Civil Asset Register and Electronic Reporting System } \\ \text { CECASE } & \text { Civil Engineering Cost and Strategy Evaluation } \\ \text { CP } & \text { Control period } \\ \text { CPN } & \text { Coloured Petri-Net } \\ \text { DCK } & \text { Deck } \\ \text { G } & \text { Good condition } \\ \text { GA } & \text { Genetic Algorithm } \\ \text { GSPN } & \text { Generalised Stochastic Petri-Nets } \\ \text { LCC } & \text { Life cycle costs } \\ \text { MGE } & \text { External Main Girder } \\ \text { MGI } & \text { Internal Main Girder } \\ \text { MK } & \text { Markov } \\ \text { MOGA } & \text { Multi-objective Genetic Algorithm } \\ \text { MONITOR } & \text { Monitor dataset } \\ \text { MTTF } & \text { Mean time to failure } \\ \text { MTTR } & \text { Mean time to repair } \\ \text { N } & \text { New condition } \\ \text { ORR } & \text { Office of Rail Regulator } \\ \text { P } & \text { Poor condition } \\ \text { PN } & \text { Petri-Net } \\ \text { SCMI } & \text { Structure Condition Monitoring Index } \\ \text { VERA } & \text { Structure Assessment Database } \\ \text { VP } & \text { Very poor condition } \\ \text { WLCC } & \text { Whole life cycle costs } \\ & \end{array}$




\section{Table of contents}

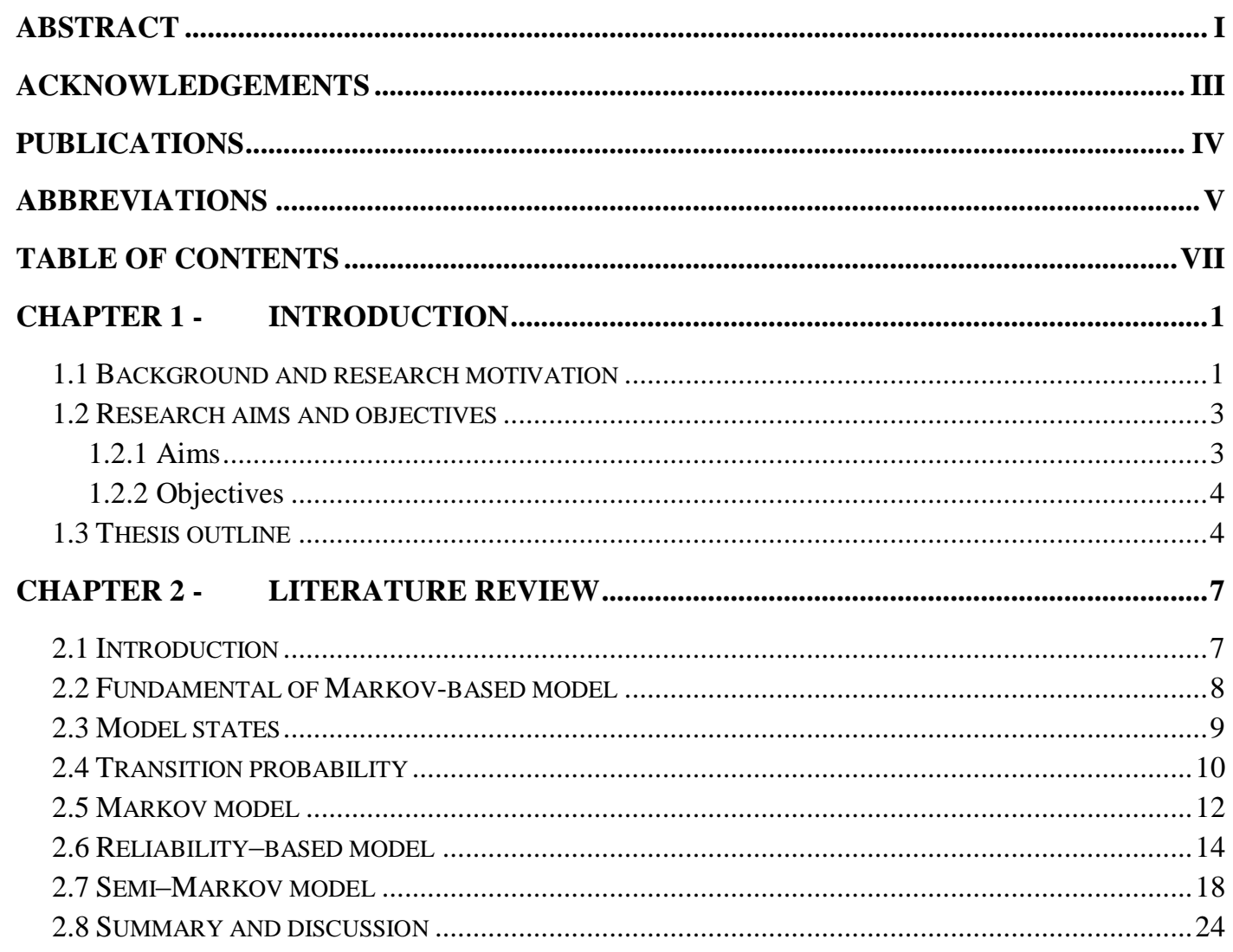

CHAPTER 3 - DATA ANALYSIS AND DETERIORATION MODELLING ....................27

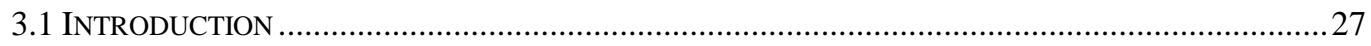

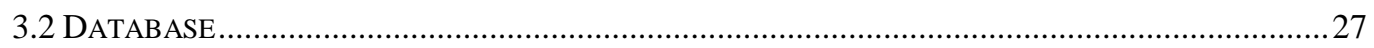

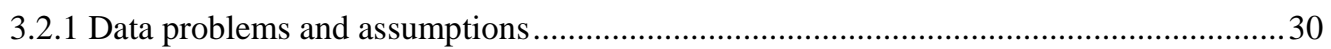

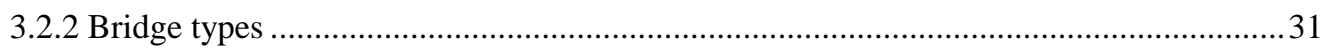

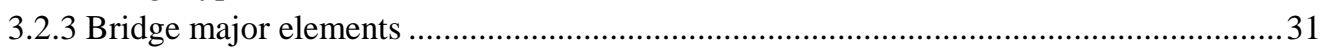

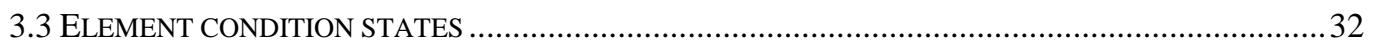

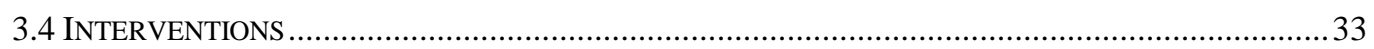

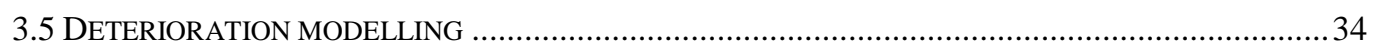

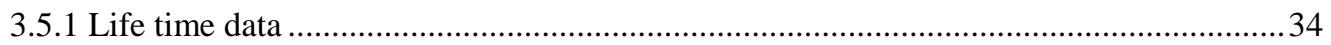

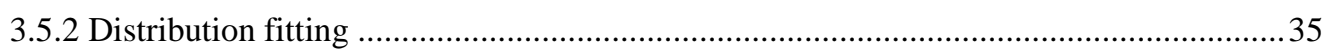

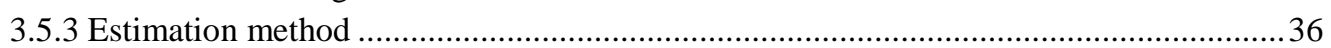

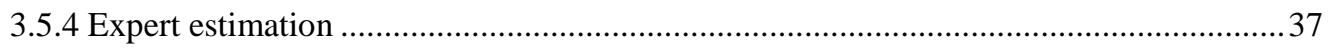

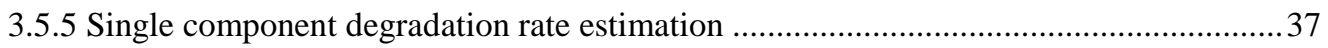

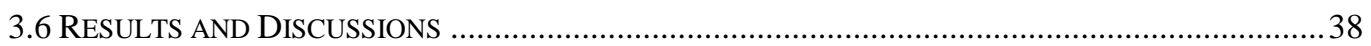

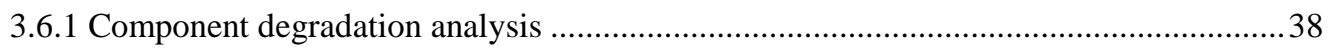

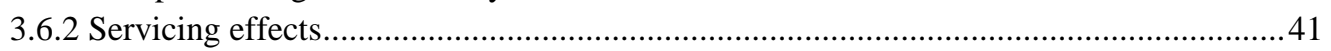

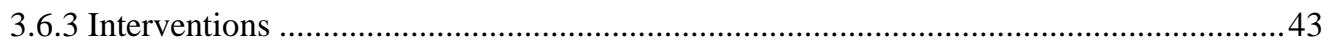

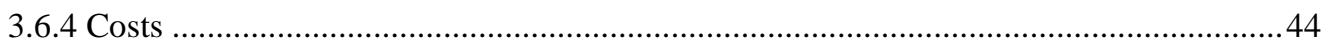

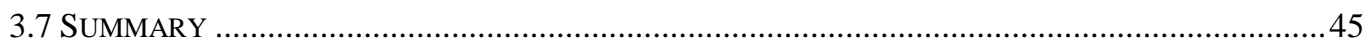

CHAPTER 4 - MARKOV BRIDGE MODEL _.......................................................................47

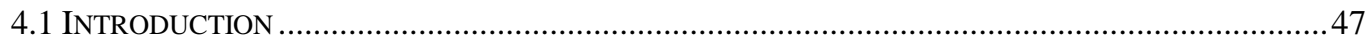

4.2 DEVELOPMENT OF THE CONTINUOUS-TIME MARKOV BRIDGE MODEL.......................................47 


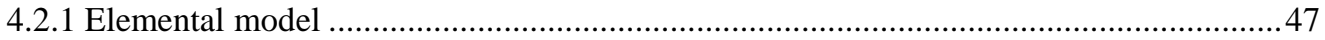

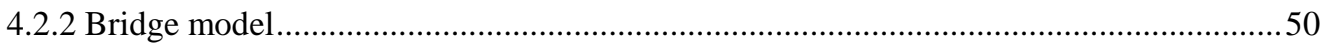

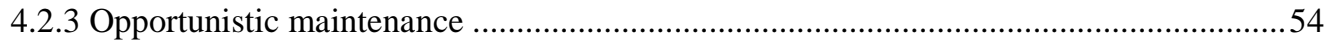

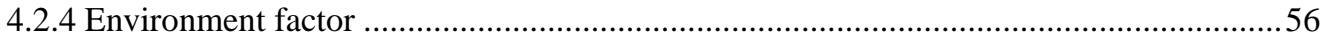

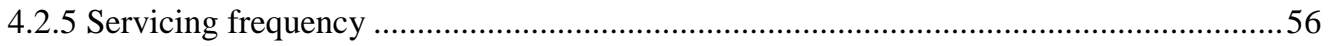

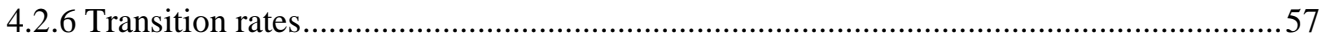

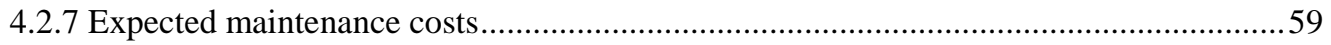

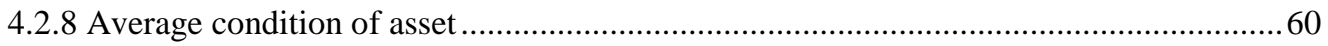

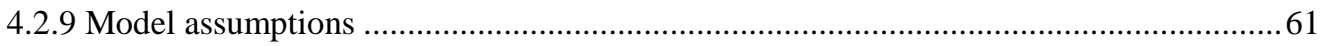

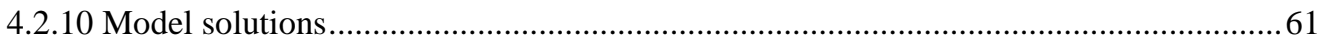

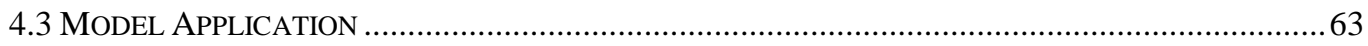

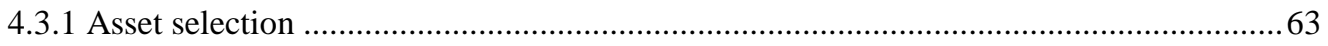

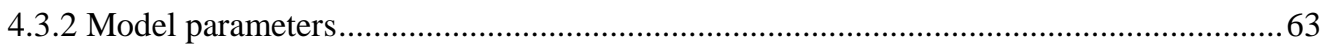

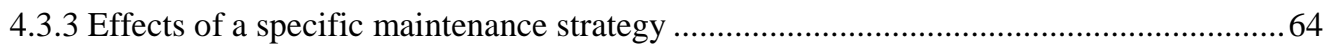

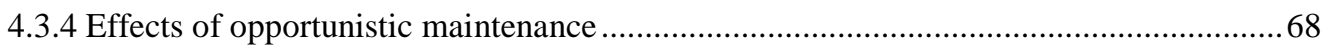

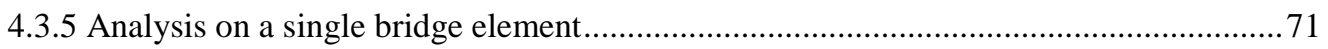

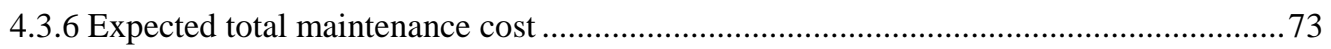

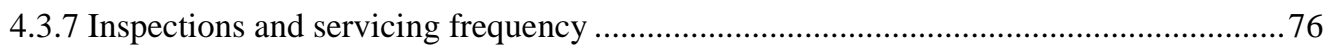

4.3.8 Scheduling of work - repair delay time ..................................................................... 77

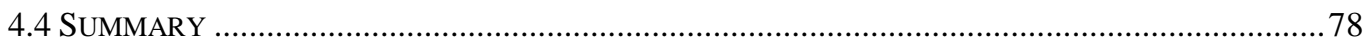

CHAPTER 5 - ～PETRI NET BRIDGE MODEL $\quad$ P............................................................81

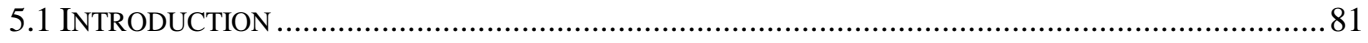

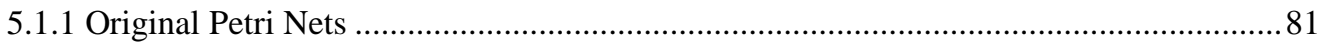

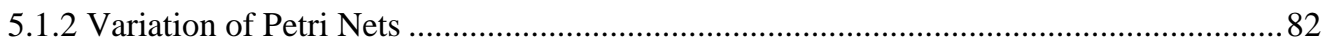

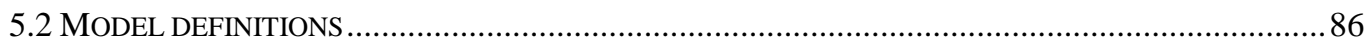

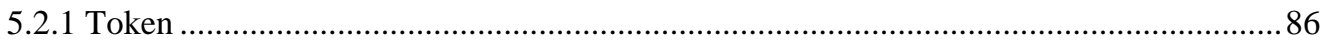

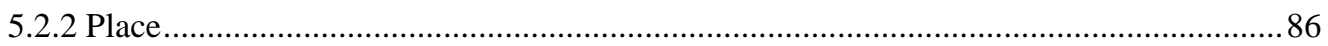

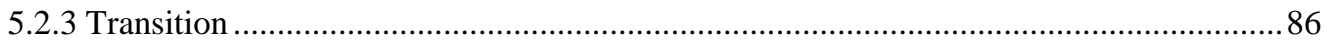

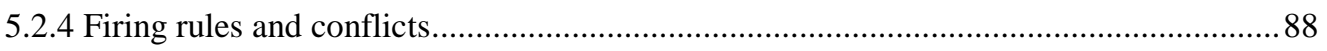

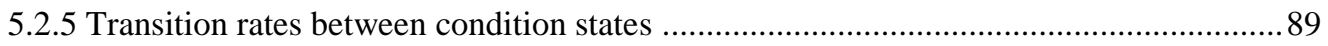

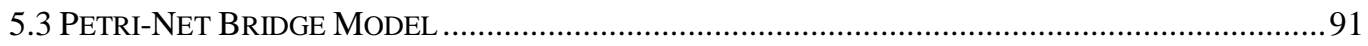

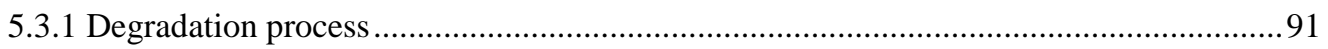

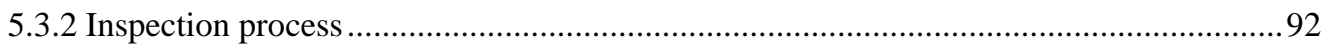

5.3.3 Maintenance schedule and delay repair process .........................................................93

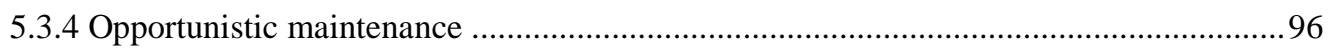

5.3.5 Modelling of the protective layer of metallic elements ..............................................97

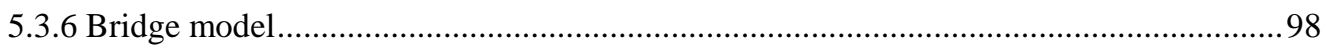

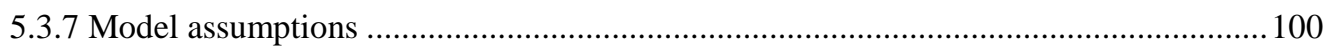

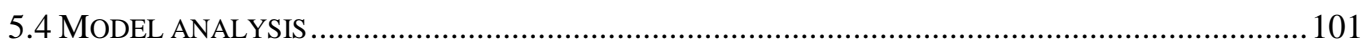

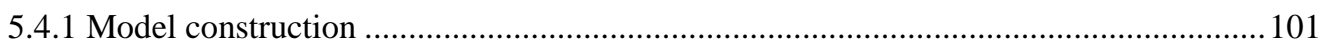

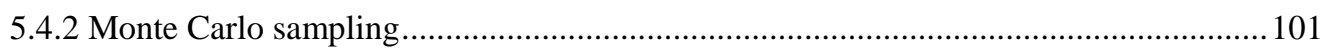

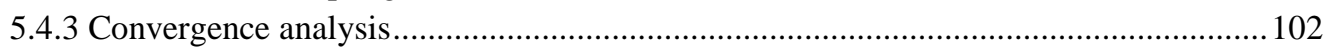

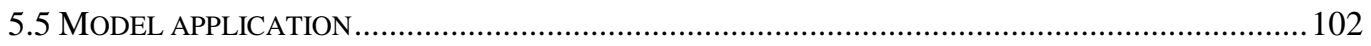

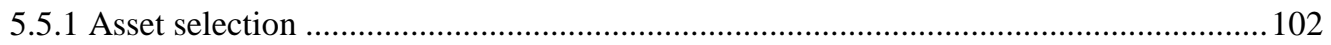

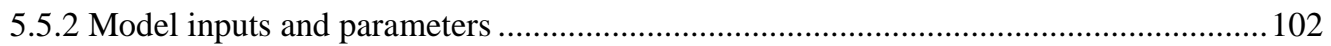

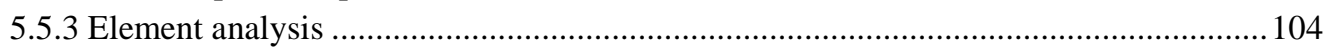

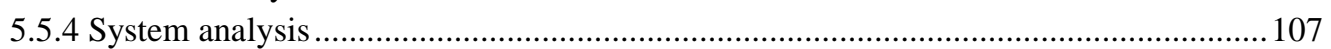

5.5.5 Effects of varying intervention strategies............................................................... 109

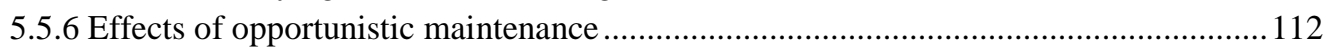

5.5.7 Effects of varying inspection and servicing interval .................................................. 114

5.5.8 Expected maintenance costs.............................................................................. 115

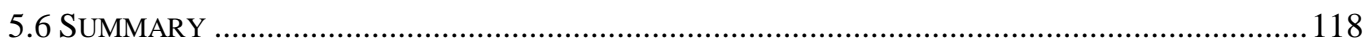


CHAPTER 6 - MARKOV AND PETRI NET MODEL COMPARISON............................119

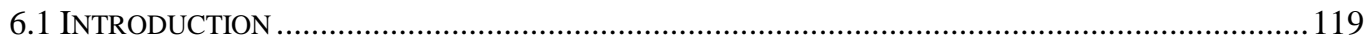

6.2 PREDICTING COMPONENT FUTURE AVERAGE CONDITION ...................................................119

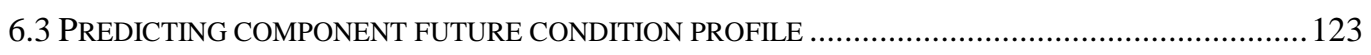

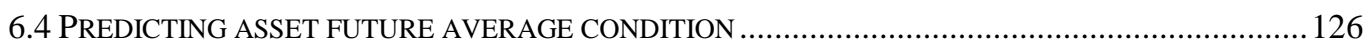

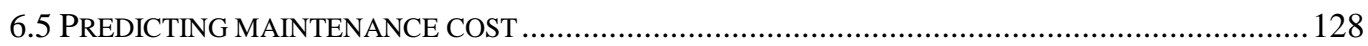

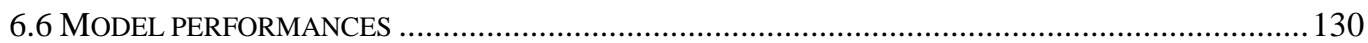

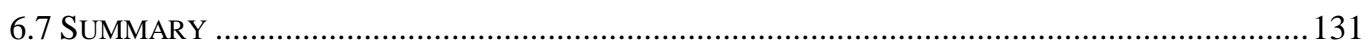

CHAPTER 7 - BRIDGE MAINTENANCE OPTIMISATION ..............................................133

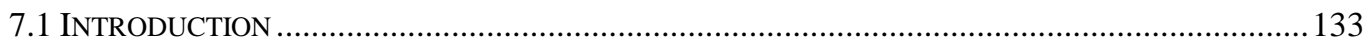

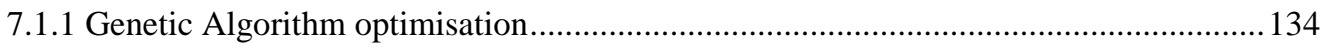

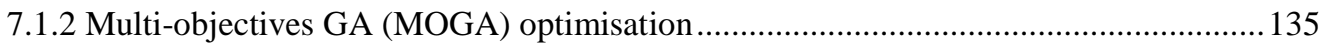

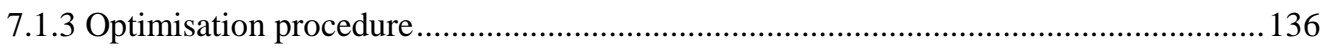

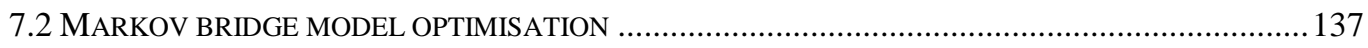

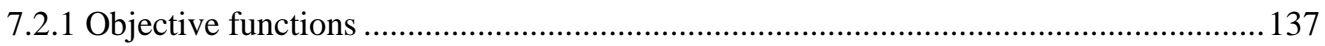

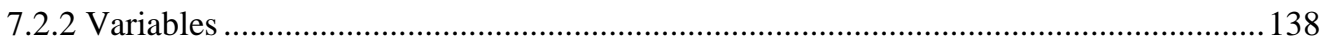

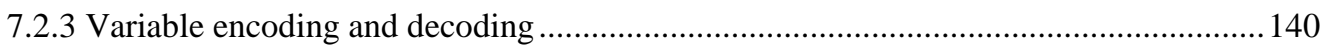

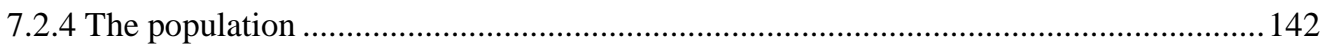

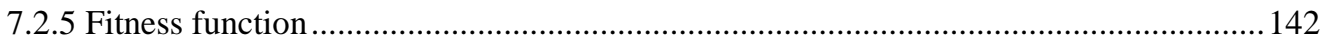

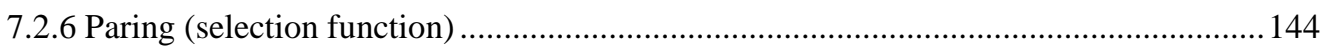

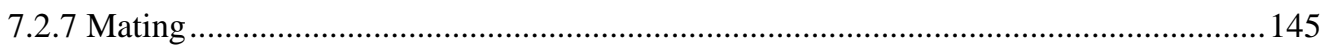

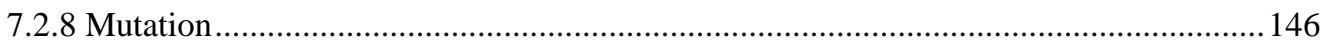

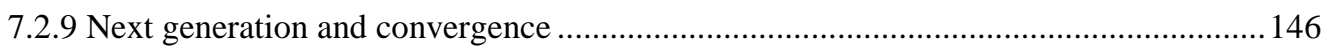

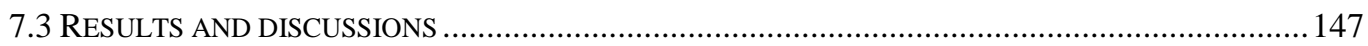

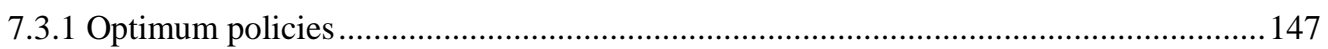

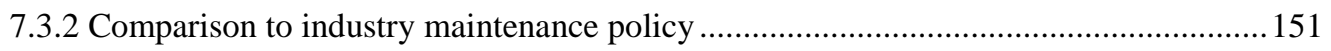

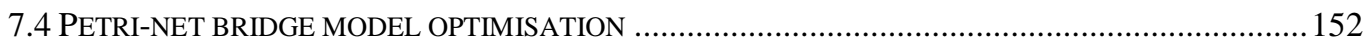

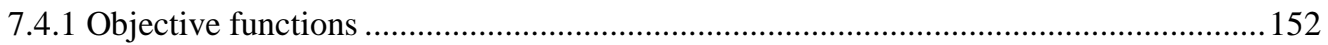

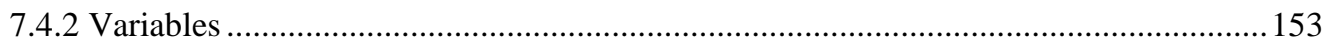

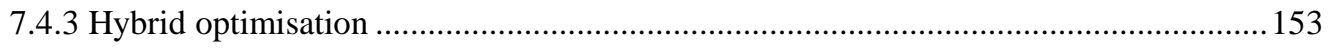

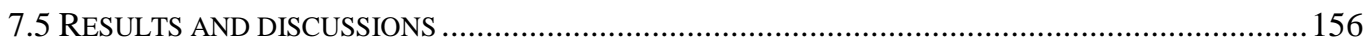

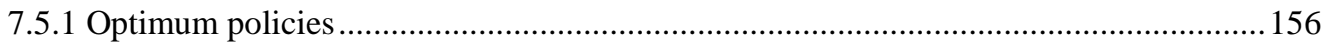

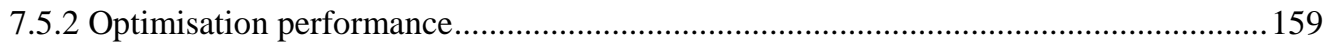

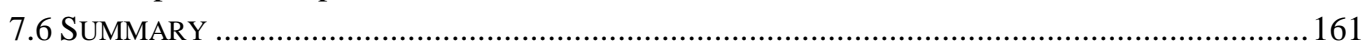

CHAPTER 8 - CONCLUSIONS ............................................................................................162

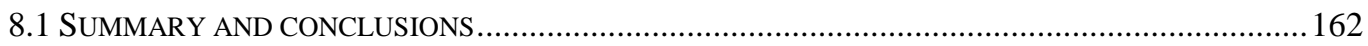

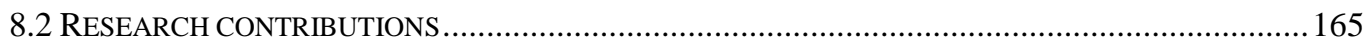

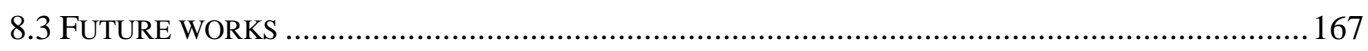

REFERENCES ..........................................................................................................................168

APPENDIX A DATA ANALYSIS AND DEGRADATION STUDY .................................174

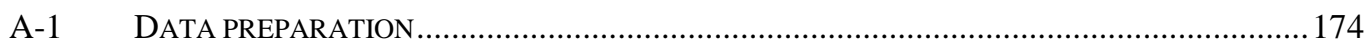

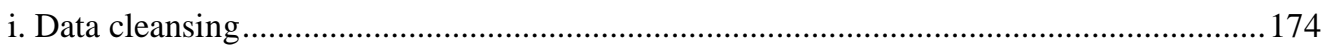

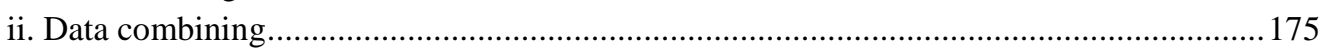

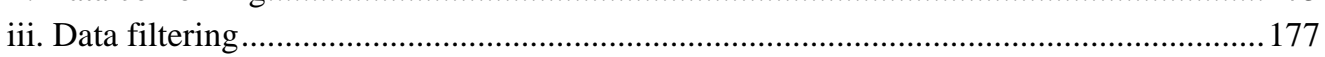

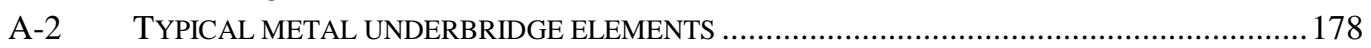

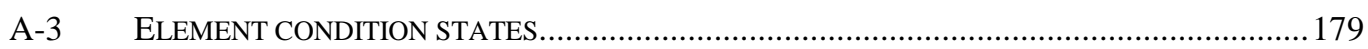

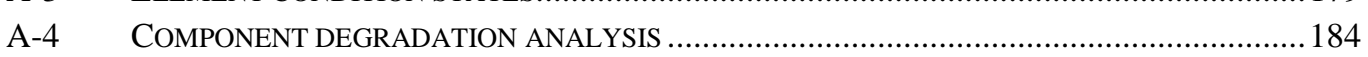

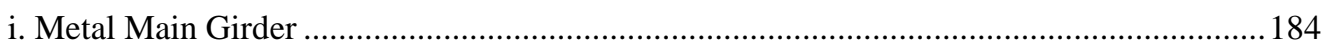

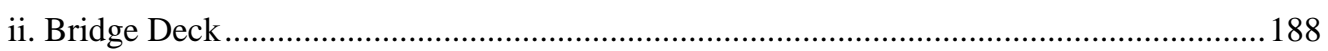


iii. Metal Deck

iv. Concrete Deck

v. Timber Deck

vi. Bearing.

vii. Abutment

APPENDIX B ENVIRONMENT ADJUSTMENT FACTOR ...................................................200

APPENDIX C MARKOV BRIDGE MODEL ...........................................................................201

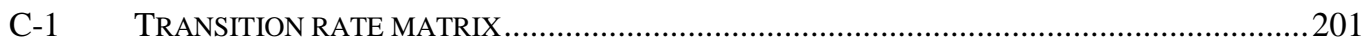

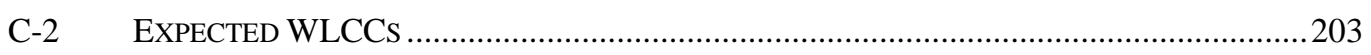

APPENDIX D PETRI-NET BRIDGE MODEL ............................................................204

D-1 DEGRADATION OF PROTECTIVE COATING AND ITS EFFECTS ON METAL ELEMENT ...........204

D-2 MODEL RESULTS - ELEMENT ANALYSIS FOR ALL BRIDGE COMPONENTS .........................2205

D-3 MODEL RESULTS - SYSTEM ANALYSIS FOR VARYING MAINTENANCE POLICIES ..............214 


\section{Chapter 1 - Introduction}

\subsection{Background and research motivation}

The function of a railway bridge is to provide a stable support to the track at an appropriate gradient and alignment along the line. Railway bridges carry the track through, over or under obstacles along the routes. Network Rail owns and maintains most of the railway bridges in the UK railway network. Bridges are classified into two main types (Network Rail, 2007b):

- Under-bridges: carry rail traffic across a geographic feature or obstruction such as a road, river, valley, estuary, railway etc.

- Over-bridges: carry another service (roadway, footway, bridleway, public utility, etc.) over the railway. This asset group includes public highways as well as accommodation and occupation bridges.

Each type of the bridge is further categorised by the main material used in their construction. Table 1.1 shows the population of all bridges under Network Rail management. Initial observation of the population is that, there is a large number of masonry bridges in the UK, almost half of the under-bridge population and a third of the over-bridge population are masonry. This is because the British railway is the oldest railway system in the world, civil structures were primarily built using bricks and masonry and the majority of the structures built many years ago, which have been strengthened and upgraded, are still in operation. Metal bridges are the second most frequent in the population as a result of a period where iron was the preferred 
choice of material for structures due to the ease, and consequently lesser time, of construction. Concrete bridges are the most popular in the over-bridge category, this is because many of the over-bridges take a highway over the railway line and concrete bridges are often the preferred choice of design for such structures. The location of most of these assets on the rail network does not allow ready access. Thus it can be difficult and costly to inspect, maintain and renew, particularly where the operation of the railway network cannot be disrupted.

\begin{tabular}{|l|c|c|}
\hline \multicolumn{1}{|c|}{ Types } & Underbridges & Overbridges \\
\hline Masonry & 11,580 & 3,970 \\
\hline Concrete & 3,021 & 4,083 \\
\hline Metal & 8,711 & 2,498 \\
\hline Other (Timber, Composite etc.) & 669 & 595 \\
\hline Total & $\mathbf{2 3 , 9 8 1}$ & $\mathbf{1 1 , 1 4 6}$ \\
\hline
\end{tabular}

Table 1.1: Population of bridges

The task of managing the bridge part of the railway infrastructure has become increasingly critical in the recent decades because of the large percentage of these assets which are deteriorating due to age, harsh environmental conditions, and increasing traffic volume. Table 1.1 shows that a large population of more than 35,000 railway bridges are currently in operation on the UK railway network. Additionally, Figure 1.1 shows that the population of bridge structures is aging with $50 \%$ of the population being more than 100 years old. Due to the unique nature of each bridge and their varied means of construction, the decision on when to maintain and what type of maintenance actions should be carried out is a very complex problem.

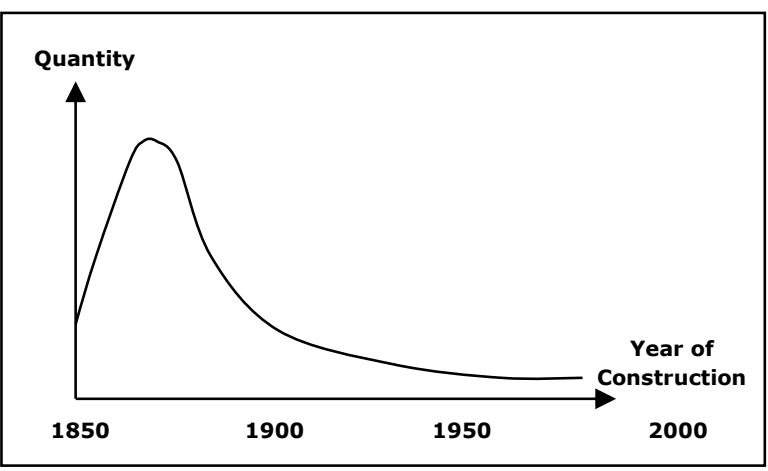

Bridge Age

Figure 1.1: Bridge age distribution (Network Rail, 2011)

The cost of maintaining these structures in an acceptable condition is significant. The annual maintenance cost for Network Rail' bridges has been estimated to be around $£ 120 \mathrm{~m}$ and is approximately a quarter of their annual maintenance expenditure 
for civil structures (Network Rail, 2013). The cost of maintenance has to be agreed with the Office of Rail Regulator (ORR) over fiveyear control periods (CP). Network Rail is required to estimate the expected maintenance, renewal and improvement costs and provide a strong justification for those figures before submission to the ORR. In the submissions for the previous control period (CP4, from 2009/10 to 20013/14), Network Rail utilised the Civil Engineering Cost and Strategy Evaluation (CECASE) tool which was developed for this purpose. The tool calculates the whole life cost, for a single asset type, for a range of possible renewal, maintenance and utilisation options. However, following critical review by the ORR, a more robust and flexible tool is required.

There is therefore a desire is to formulate a bridge model and a decision making process which will enable a strategy to be established which will enable assets to be maintained and renewed to minimise the whole life costs. The quality of the decisions made with such an approach is dependent upon how well the deterioration processes of the assets over time are understood. Historical data can be used to study the degradation process of bridge elements. Models can be formulated to predict the future condition of bridge asset along with the effect that interventions such as servicing, repair, and element replacement will produce. Intervention costs can then be integrated into the model and an optimisation can be performed to determine the optimum maintenance strategy indicating what actions need to be taken at what time and where in order to minimise the whole life spend whilst providing an acceptable service and safety performance.

\subsection{Research aims and objectives}

\subsubsection{Aims}

The goal of the research presented in this thesis is to develop complete bridge asset models. The focus is on accurate prediction of the future asset condition at both the whole asset and component levels. Maintenance is then incorporated into the model to demonstrate the effect of different intervention strategies. An optimisation is then performed to support the decision making process to establish the optimum maintenance policy.

The principle goals of the research are: 
- Examine the historical data records for the maintenance actions that have been carried out on Network Rail bridges of similar material and construction.

- Establish a bridge model to estimate how much assets deteriorate over time.

- Estimate the future work volume for the whole asset at elemental level.

- Predict an optimal strategy for maintenance (servicing, repair, replacement) in order to minimise the whole life costs.

\subsubsection{Objectives}

The principle objectives of this research project are:

- Develop a novel deterioration model that does not use the current condition rating system, the model would use information provided from the historical maintenance records to understand what interventions have been carried out at different stages of an asset's life.

- Develop a bridge model based on the widely accepted Markov modelling approach. The model will take into account different factors that affect the deterioration and maintenance planning process. Thus the model is considered in much more detail than other bridge models available in the literature.

- Develop a bridge model based on an approach which is novel for bridge condition prediction - the Petri-Net modelling approach. The improvements and advantages along with the disadvantages of the method over the traditional modelling approach will be identified and discussed.

- Optimise the bridge maintenance strategy to minimise the whole life costs whilst providing an acceptable condition state using the Genetic Algorithm technique.

\subsection{Thesis outline}

The thesis is organized as follows:

Chapter 2 provides a detailed review of the literature reporting the previous research conducted on modelling the degradation process of bridges and bridge elements. Different modelling approaches are reviewed and their advantages and disadvantages are critically appraised. 
Chapter 3 presents a novel method of modelling the asset deterioration process, this involves constructing a timeline of all historical work done of a bridge element and analysing the time of the component reaching different intervention actions in order to establish a component lifetime distribution. The analysis methodology is presented in detail following a discussion of the available datasets. The results obtained from the analysis are also presented.

Chapter 4 demonstrates a Markov modelling approach to predict the condition of individual bridge elements along with the effects that interventions will produce. The development of the bridge model is also discussed and simulation results are presented to demonstrate the capability of the model as well as the type of information the model generates that can be used to support the asset management strategy selection.

Chapter 5 describes the development of a bridge model using the Petri-Net (PN) modelling technique. This chapter gives an overview of the PN method before developing a PN bridge model. It also discusses, in detail, the modifications to the original PN modelling technique to suit the problem of modelling bridge asset condition. The model is then applied to a selected example asset and simulation results are presented and discussed.

Chapter 6 compares the two bridge models developed in term of model results and performance.

Chapter 7 presents an optimisation framework based on the Genetic Algorithm technique as a decision making approach to select the best maintenance strategy. A high performance hybridoptimisation was applied using both the Markov and the Petri-Net bridge models. The optimisation is a multi-objective optimisation that looks for the maintenance policies that will produce the lowest expected maintenance cost whilst maximising the average condition of the asset.

Chapter 8 summarises the research work, highlights its contributions, and gives recommendations for future research. 


\section{Chapter 2 - Literature Review}

\subsection{Introduction}

Over the last few decades, numerous papers have appeared in the literature, which deal with the modelling of bridge asset management. These studies focused on developing deterioration models that predict the degradation rates and the future states of a bridge. These models, reported in this section, use a variety of techniques. The simplest form of bridge deterioration modelling is the deterministic model. Deterministic models predict the future asset conditions deterministically by fitting a straight-line or a curve (Jiang and Sinha, 1989, Sanders and Zhang, 1994) to establish a relationship between the bridge condition and age. Due to the nature of deterministic models that they do not capture the uncertainty in the data, many studies develop stochastic models which are considered to provide improved prediction accuracy (Bu et al., 2013). In stochastic models, the deterioration process is described by one or more random variables, therefore this method takes into account the randomness and uncertainties that arise in the processes that are being analysed. Amongst the deterministic models, regression analysis is a methodology widely used, whereas, the Markovbased model is considered as one of the most common stochastic techniques adopted. This section focuses on all the studies using the stochastic approach, starting with the fundamentals of Markov models before exploring Markov-based and other probabilistic bridge models available in the literature.

It is important to note that even though some of the reported models were applied to railway bridges, some were used to 
predict the deterioration rate and future state of highway bridges. From the asset management point of view, the two situations differ from each other since they could be managed by different authorities e.g. Network Rail and Highway Agency in the UK. However, in terms of methods and techniques to predict the future state of a bridge, there are no differences as the deterioration models for railway bridges can be applied for highway bridges and vice-versa. This is because the two models are essentially based on the same mathematical or statistical techniques. The purpose of this section is to study all the methods and techniques, reported in the literature to model the deterioration of bridges. By considering models for highway bridges and other infrastructure assets, a broader range of techniques can be studied.

\subsection{Fundamental of Markov-based model}

The Markov approach can be used for systems that vary discretely or continuously with respect to time and space (Andrews and Moss, 2002). For the Markov approach to be applicable, the system must satisfy the Markov 'memory-less' property, that is that the probability of a future state in the process depends only on the present state and not on the past states (Ibe, 2009). This property can be expressed for a discrete state parameter $\left(X_{t}\right)$ in a stochastic process as:

$$
\begin{gathered}
P\left(X_{t+1}=i_{t+1} \mid X_{t}=i_{t}, X_{t-1}=i_{t-1}, \ldots, X_{1}=i_{1}, X_{0}=i_{0}\right) \\
=P\left(X_{t+1}=i_{t+1} \mid X_{t}=i_{t}\right)
\end{gathered}
$$

where

$\mathrm{i}_{\mathrm{t}}=$ state of the process at time $t$;

$\mathrm{P}=$ conditional probability of any future event given the present and past event.

Markov chains are then used as performance prediction models for bridge assets or bridge components by defining discrete condition states and accumulating the probability of transition from one condition state to another over multiple discrete time intervals. Transition probabilities are represented by a matrix of order $(n \times n)$ called the transition probability matrix $(P)$, where $n$ is the number of possible condition states. Each element $\left(p_{i j}\right)$ in this matrix represents the probability that the condition of a bridge component will change from state (i) to state $(j)$ during a unit time interval called the transition period. If the Markov model 
is continuous in time, a different equation formulation is required with the transitions being represented by rates. Almost all of Markov-based bridge models in the literature are discrete and use transition probabilities. If the initial condition vector $P(0)$ that describes the present condition of a bridge component is known, the future condition vector $P(t)$ at any number of transition periods $(t)$ can be obtained as follows:

$$
P(t)=P(0) \times P^{t}
$$

where

$$
P(t)=\left[\begin{array}{cccc}
p_{1,1} & p_{1,2} & \ldots & p_{1, n} \\
p_{2,1} & p_{2,2} & \ldots & p_{2, n} \\
\ldots & \ldots & \ldots & \ldots \\
p_{n, 1} & p_{n, 2} & \ldots & p_{n, n}
\end{array}\right]
$$

Following the formulation of a Markov model, the analysis will yield the probability of being in any of the model states. In the problem of modelling the bridge degradation process, the system 'failure' probability is determined by summing the probabilities of residing in the states which represent an asset 'failure' condition. Note that the term 'failure', used here and also throughout this research, does not mean the physical failure of a component but indicates an event when an intervention is required or when the component has reached a specified threshold condition.

\subsection{Model states}

States of bridges or bridge elements are usually allocated discrete numbers that are associated with a specific condition. Thus, the model state is usually defined corresponding to these defined condition rating systems (e.g. good, fail, poor, etc.). There are, however, some models which reduce the number of model states by choosing a threshold condition that is considered worst in the model but not necessarily the worst condition recorded in the condition rating system. For example, a condition rating 7 is considered worst state in the deterioration model, however there are 9 condition states recorded (Scherer and Glagola, 1994). Table 2.1 shows the typical model states that have been used by studies in the literature. It can be seen that there are no more than 10 model states used. Robelin and Madanat (2007) argued that, this is to keep the model computationally efficient. This is especially true when using Markov approach, since the statespace of the model would explode exponentially with the increasing number of modelled components. 


\begin{tabular}{|c|l|l|l|}
\hline $\begin{array}{c}\text { Number } \\
\text { of states }\end{array}$ & $\begin{array}{c}\text { Rating } \\
\text { system }\end{array}$ & \multicolumn{1}{|c|}{ State description } & \multicolumn{1}{|c|}{ References } \\
\hline 10 & $0,1, \ldots, 8,9$ & $\begin{array}{l}\text { Failed, imminent failure... } \\
\text { very good, excellent } \\
\text { condition. }\end{array}$ & $\begin{array}{l}\text { (Jiang and Sinha, 1989, } \\
\text { Ng and Moses, 1996, } \\
\text { Cesare et al., 1992, } \\
\text { Mishalani and Madanat, } \\
\text { 2002) }\end{array}$ \\
\hline 7 & $3,4, \ldots, 8,9$ & $\begin{array}{l}\text { Poor, marginal... good, } \\
\text { new }\end{array}$ & $\begin{array}{l}\text { (Scherer and Glagola, } \\
1994)\end{array}$ \\
\hline 6 & $1,2, \ldots, 5,6$ & $\begin{array}{l}\text { Critical, urgent... good, } \\
\text { very good }\end{array}$ & (Morcous, 2006) \\
\hline 7 & $1,2, \ldots, 6,7$ & $\begin{array}{l}\text { Potentially hazardous, } \\
\text { poor... good, new }\end{array}$ & $\begin{array}{l}\text { (DeStefano and Grivas, } \\
\text { 1998) }\end{array}$ \\
\hline 4 & $6,7,8,9$ & $\begin{array}{l}\text { Satisfactory, good, very } \\
\text { good, excellent condition }\end{array}$ & (Sobanjo, 2011) \\
\hline 7 & $1,2, \ldots, 6,7$ & $\begin{array}{l}\text { Totally deteriorated, } \\
\text { serious deterioration... } \\
\text { minor deterioration, new } \\
\text { condition }\end{array}$ & $\begin{array}{l}\text { (Agrawal and } \\
\text { Kawaguchi, 2009) }\end{array}$ \\
\hline 5 & $1,2,3,4,5$ & Good as new... failure & (Kleiner, 2001) \\
\hline 5 & $1,2,3,4,5$ & $\begin{array}{l}\text { Do nothing, preventative, } \\
\text { corrective maintenance, } \\
\text { minor, major } \\
\text { rehabilitation }\end{array}$ & (Yang et al., 2009) \\
\hline
\end{tabular}

Table 2.1: Example of model states employed in literature

The bridge model that was developed and used by Network Rail to manage railway bridge assets is based on the Markov approach. The model states are defined based on the Structure Condition Marking Index (SCMI) which was developed (Network Rail, $2004 \mathrm{~b}$ ) to rate the condition of an asset taking values ranging from 0 to 100 . Depending on a particular asset, the bridge model used by Network Rail has either 10 or 20 states, these states corresponds to 10 or 20 condition bands, each representing 10 or 5 SCMI scores.

\subsection{Transition probability}

The transition probability is the probability of moving between different condition states of a bridge or a bridge component. The transition probability reflects the degradation process and directly affects the accuracy in the model prediction. Therefore, for Markov models, generating the transition probability is a key component (Mishalani and Madanat, 2002). There are two methods commonly used to generate the transition probability matrix from the bridge condition ratings data: regression-based optimisation (expected value) method, and percentage prediction (frequency) method. 
The regression (expected value) method (Morcous, 2006, Butt et al., 1994) estimates transition probabilities by solving the nonlinear optimisation problem that minimises the sum of absolute differences between the regression curve that best fits the condition data and the conditions predicted using the Markov chain model. The objective function and constraints of this optimisation problem can be formulated as follows:

$$
\min \sum_{n=1}^{N}|C(t)-E(t)|
$$

where:

$N$ is the total number of transition period;

$C(t)$ is the condition at transition period number $\mathrm{t}$ based on the regression curve;

$E(t)$ is the expected condition at transition period

number $t$ based on Markov chain;

$E(t)=P(t) \cdot S$

$P(t)$ is the transition probability matrix

$S$ is the vector of condition state

The percentage (frequency) method is quite commonly used (Jiang and Sinha, 1989, Ortiz-García et al., 2006). In this method, the probability of transitioning between states is estimated using:

where:

$$
p_{i j}=\frac{n_{i j}}{n_{i}}
$$

$\mathrm{n}_{\mathrm{ij}}$ is the number of bridges originally in state $\mathrm{i}$ which have moved to state $\mathrm{j}$ in one step;

$n_{i}$ is the total number of bridges in state i before the transition.

In between these two techniques, the frequency approach requires at least two sets of inspection data without any maintenance interventions, for a large number of bridge components at different condition states. In the regression approach, only one set of data is needed, condition ratings are plotted against age. And the transition probabilities are then estimated by associating the regression function with the transition matrix. This involves seeking an optimal solution to minimise the difference between the expected condition rating (from regression function) and that derived from the transition 
matrix. Therefore the frequency approach was usually used when the data is available.

It is realised that the bridge condition databases used in all the studied in the literature do not accommodate these methods. These databases would normally, at best, contain records that only go back as far as the last two decades, the condition ratings usually do not change significantly during short-term periods. Moreover, the database would usually be filtered to remove data indicating a rise in condition rating (due to the effect of improvement or maintenance), this further reduces the available data for the analysis. All these factors exhibit the inaccuracy in the determination of the transition probability which directly affects the prediction of future asset condition.

\subsection{Markov model}

The Markov approach is the most common stochastic techniques that has been used over 20 years in modelling the deterioration of bridges and bridge elements.

Jiang and Sinha (1989) were one of the first to demonstrate the use of a Markov model in predicting the deterioration rate of bridges. Their paper focused on discussing the methodology in estimating the transition probability based on the condition score data of bridges at different ages. The method used in this paper is the expected value method. Although the paper has demonstrated the method, there was no real application on actual bridge condition data.

Cesare et al. (1991) describe methods for utilising Markov chains in the evaluation of highway bridge deterioration. A study was carried out based on the empirical data of 850 bridges in New York State. The data contains bridge element condition ratings on the scale of 1 to 7 with 7 being new and 1 being the worst condition. Assuming the deterioration of each element is independent of all other bridge elements, the Markov model was then applied to predict the evolution of the average condition rating of a set of bridges and the expected value of condition rating for a single bridge. The paper discussed that, the effects of the lack of supporting data would require the results produced in this paper to be further validated and suggested that the Markov model employed would need more data in order to produce accurate results. 
Scherer and Glagola (1994) explored the applicability of the Markov approach on the modelling of the bridge deterioration process. The authors developed a Markov bridge model for a single bridge asset with the intention of using a single model to manage the entire population of 13,000 bridges in the state of Virginia, USA. An individual bridge is considered to have 7 states. It was pointed out that the Markov model size would be computationally intractable even for a population of 10 bridges since the model size would be $7^{10}$ (approximately $300,000,000$ states). To tackle this problem, the author developed a classification system which group bridges according to: route type (interstate, primary, secondary); climate; traffic loading; bridge type (concrete, timber deck); bridge spans (single, multiple); bridge age (0-20, 21-40, 41 years and older). A 7-state Markov model was then developed that was representative of a bridge in each bridge class. In summary, the paper addressed the issues in state-space combinatorial explosion associated with Markov models, and verified that the Markov assumption is acceptable in bridge asset management modelling.

Morcous (2006) also adopted Markov chain models for predicting the future condition of bridge components. A study was carried out to predict the condition of a bridge deck using the data from 9,678 structures of 57 different types of highway structure in Quebec, Canada. The data includes 500,924 inspection records from 1997 to 2000 that recorded the structure condition index from 0 to 100 with the greater the condition the better. The paper highlighted several assumptions used in the model such as: bridge inspections are performed at pre-determined fixed time intervals (constant inspection period); future bridge condition depends only on present condition and not the past condition. The transition probabilities were determined using the frequency method. Data with increased condition ratings were removed to eliminate the effects of maintenance on the data. The paper investigated that the inspection period which was not constant, and follows a normal distribution. The effects were found by adjusting the developed transition probabilities for the variation in the inspection periods and the predicted performance before and after adjustments. The adjusted rates used Bayes' rules, and the paper shows that the variation in the inspection period may result in a $22 \%$ error in predicting the life of component. 
All of the Markov models presented are simple, they predict the future condition for a single bridge component or a whole bridge. Complete bridge asset model, that describes a complete bridge structure including bridge elements, was not developed. In additional, Markov-based model cannot efficiently consider the interactive effects of the deterioration rates between different bridge elements (Sianipar and Adams, 1997).

\subsection{Reliability-based model}

The degradation process of a bridge element can be modelled using the 'life data' analysis technique. This technique is popular in system reliability studies. DeStefano and Grivas (1998) demonstrated that the 'life data' analysis method can be applied for the development of probabilistic bridge deterioration models on the basis of the available information. The data required using this technique is the times to a specified transition event. This event is found by looking at the change in the condition rating, i.e. a drop in condition score, for a bridge element recorded in the database. This technique considers both complete and censored lifetime data. Complete data indicates the transition time associated with state transition events that have been observed. Censored data indicates the state transition event that has not been observed within the analysis period, this might be because the component was replaced while in its initial condition state or the analysis period is not long enough for the transition to occur.

In DeStefano and Grivas (1998) paper, the Kaplan and Meier method is applied to these data to calculate non-parametric estimates of cumulative transition probability corresponding to transition times and specific transition events. The degradation process is described by the transition probability which is defined as this cumulative probability. A study was carried out using the inspection data of 123 bridges in New York, USA. The paper suggested that the reliability-based method recognises the 'censored' nature of bridge inspection data and incorporates these data into the deterioration modelling process. The paper also pointed out the subjective nature of inspection data and the inherent error this adds to the model. The paper demonstrated the approach by analysing the bridge component lifetime data, although it is suggested that better fitting and more robust distributions could be used to fit to the data. 
Frangopol et al. (2001) used a reliability index ( $\beta$ ) that measures the bridge safety instead of condition. The reliability index was previously developed by Thoft-Christensen (1999) and is defined in Table 2.2. The deterioration rate of a bridge is now the deterioration rate of the reliability index.

\begin{tabular}{|l|c|c|}
\hline State & State no & $\boldsymbol{\beta}$ \\
\hline Extremely good & 5 & $>10$ \\
\hline Very good & 4 & {$[8,10]$} \\
\hline Good & 3 & {$[6,8]$} \\
\hline Acceptable & 2 & {$[4.6,6]$} \\
\hline Non-acceptable & 1 & $<4.6$ \\
\hline
\end{tabular}

Table 2.2: Definition of the bridge reliability index (Thoft-Christensen, 1999)

The deterioration process is modelled in different phases and the authors used different distributions to model the times of these phases. In Figure 2.1, taken from this paper, the authors demonstrate a typical degradation process of a bridge. The reliability index starts at a certain level, this is modelled using a normal distribution (denoted as process a). The reliability index stays the same for a certain time before the index starts dropping, this time is modelled using a Weibull distribution (process b). The rate of deterioration of the index is modelled with normal distribution (process $\mathrm{c}$ ). Then a repair is carried out which increases the index and the deterioration process starts again. These distributions are defined according to expert opinion and a Monte Carlo simulation is used to generate random sample from these pre-defined distributions.

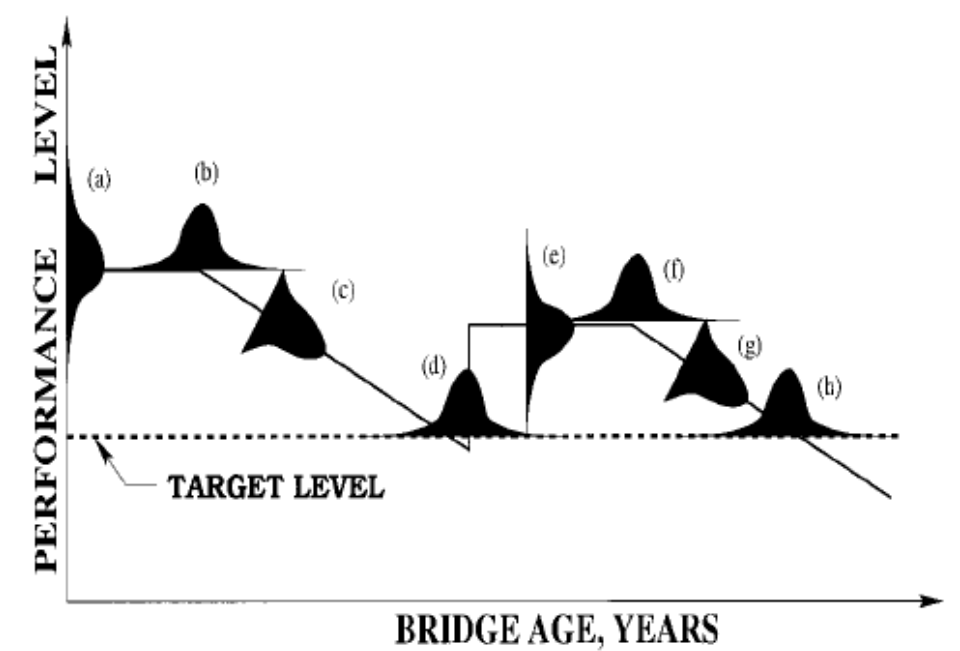

Figure 2.1: Typical deterioration process phases and distributions used for each phase (Frangopol et al., 2001) 
The authors have developed a reliability index and believed that it has the added benefit to measure the deterioration process, however the benefit cannot be seen. Moreover, the index is an indicator for a bridge or a group of bridges and thus will have no indication of the condition of the bridge elements. The distribution parameters used in the paper are defined based on expert judgement, this leads to inaccuracy in the degradation model. It is realised that, with the flexibility of the demonstrated approach, there are many scenarios which can be modelled. However, a different degradation model would be required for each scenario. The model also would require a large computation time.

Noortwijk and Klatter (2004) performed a statistical analysis on the life time of a bridge by fitting Weibull distribution to the lifetimes of demolished bridges (complete lifetime) and current ages of existing bridges (censored/incomplete lifetime). A distribution of the times between when the bridge was built and when the bridge is renewed was obtained from this study. There are, however, several issues with the study. Firstly, the data type does not reflect the demolishing and replacement of bridges due to deterioration failure but due to the change in requirement. Secondly, only one 'failure' mode is considered which is the complete failure of the bridge.

Sobanjo et al. (2010) presented a study in understanding the natural deterioration process (without significant improvement) of bridges in Florida, USA. The authors studied the failure times (time to reach a specified condition threshold) and sojourn times (time of staying in one condition state) at various condition states. The time (age) of the bridge at transition was assumed to be the average of two estimates: the age at departure and age at arrival at a given condition state. Weibull distribution was reported as a best fit to model the uncertainties in the failure times obtained. The study was on bridge decks and superstructures using the NBI (National Bridge Inspection) database with the bridge condition reports from 1992 to 2005. Note that this database is used in the USA and there are 9 condition ratings for a bridge component. The assumption used in this paper is that no major improvement is done to the bridge, thus data which indicates improvement, i.e. increase ratings between consequent inspections, are subsequently removed. The Weibull shape parameters reported from this study are generally 
quite high (larger than 2), this means that the results produced by the paper should be further verified. Despite few limitations, the paper demonstrated that, with the shape parameters larger than 1 , the deterioration rate of bridge element is increasing over time.

Agrawal and Kawaguchi (2009) carried out an extensive empirical study using this method on major bridge components. The historical data used was collected from 17,000 highway bridges in New York State, between 1981 and 2008. The data contains bridge component condition ratings from 1 to 7 with 7 being new and 1 being in failed condition. Data where ratings improved were removed from the study. The paper describes an approach to fit a Weibull distribution to the durations (in number of years) that an element stays in a particulate condition rating. The mean time of staying in a condition rating is then statistically derived from the distribution obtained. These means are then plot on a graph of condition ratings vs. age (years) and a $3^{\text {rd }}$ degree polynomial curve is fitted to show the deterioration rate. The deterioration curves and equations are shown in Figure 2.2 and Table 2.3.

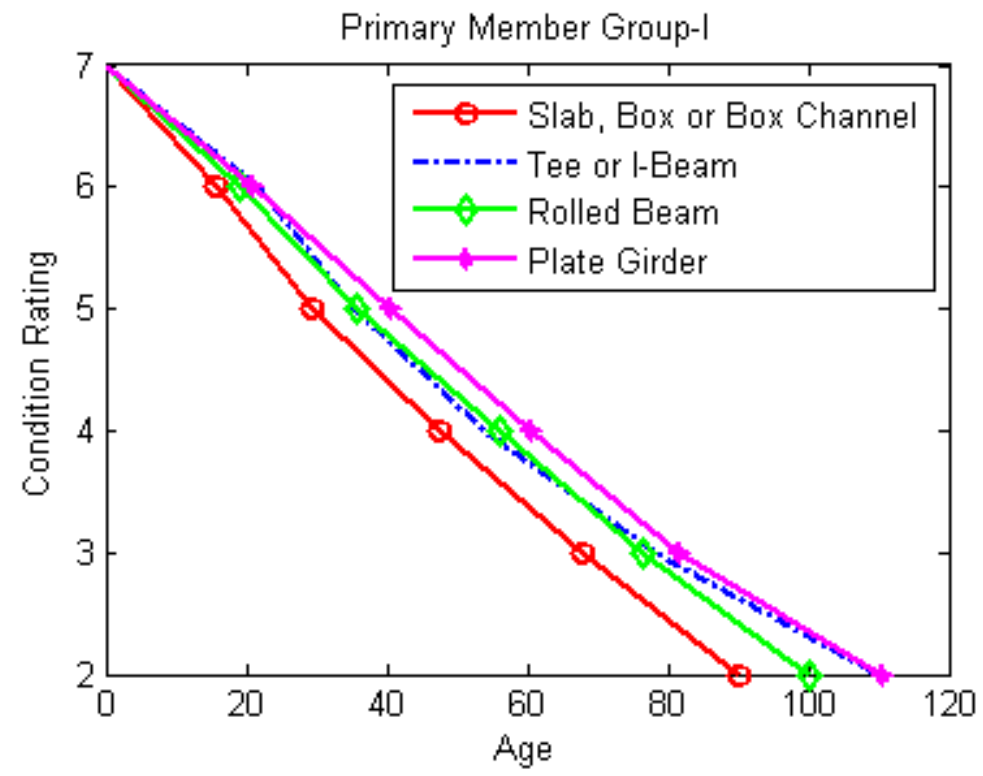

Figure 2.2: Condition rating against age (Agrawal et al., 2010)

\begin{tabular}{lcc}
\hline Element & Design types & Deterioration equation \\
\hline Superstructure recommendation & Group-I & Slab \\
& Stringer/multibeam or girder & CR $=7-0.0550966 T-0.00000107 T^{2}+0.0000005 T^{3}$ \\
& Girder and floor beam system & $\mathrm{CR}=7-0.0608104 T+0.0001228 T^{2}-0.0000002 T^{3}$ \\
& Tee beam & $\mathrm{CR}=7-0.0375553 T-0.0003374 T^{2}+0.0000019 T^{3}$ \\
& Box beam or box girder & $\mathrm{CR}=7-0.0334694 T-0.0005675 T^{2}+0.0000041 T^{3}$ \\
& & $\mathrm{CR}=7-0.0671339 T+0.0000287 T^{2}+0.0000015 T^{3}$
\end{tabular}

Table 2.3: Deterioration equation as a function of condition rating and age (Agrawal et al., 2010) 
In a later study (Agrawal et al., 2010), the authors compared the deterioration curves produced by this method and those produced by applying the Markov approach. The comparisons were on the bridge primary members such as: girder, deck, pier cap, abutment or pier bearing, abutment. The paper reports that the deterioration rates produced by the Weibull approach are generally higher than those resulting from the Markov approach. The values of shape parameter were greater than one which clearly shows the bridge component deterioration rate is durational dependent and the consideration of censored data illustrated that the Weibull approach provides a better fit to the observed bridge element conditions. The authors have demonstrated the reliability approach on the modelling of the bridge component degradation process. The mean time to different condition states is obtained from the fitted Weibull distribution. However the fitting of a polynomial curve to these means to describe the degradation process eradicates the advantage that the reliability-based method presents. This is because the mean time will not fully explain the degradation process described by a Weibull distribution.

It was found that, for all the studies which employed the reliability-based approach, the data used in these studies are the condition ratings. The data required for the analysis is the time to an event where the element condition changes, it was then assumed that the transition events occur at the midpoint between inspection dates. This reason is that the actual date when a transition event happens is not observed. This assumption is invalid when the inspection intervals are wide relatively to the assumed distribution width. This introduces bias in the duration times that lead to errors in the accuracy of the modelled degradation process.

\subsection{Semi-Markov model}

The Markov model is based on the assumption of exponential distribution for duration for the sojourn/holding times at each specific bridge condition. This property, when used in modelling bridge deterioration, suggests that the probability for a bridge to move from its current state to another more deteriorated state is constant in the discrete period of time considered and does not depends on how long it has been in the current state. SemiMarkov models use different distributions (most of the papers 
discuss in this section used Weibull distribution) to model these duration times of a bridge staying in a specific condition. The derivation of the duration times and the fitting of the Weibull distribution to these times is very similar to the method discussed previously for reliability-based models.

The semi-Markov process could be conceived as a stochastic process governed by two different and independent randomgenerating mechanisms. Let $T_{1}, T_{2}, \ldots, T_{n-1}$ be random variables denoting the duration times in states $1,2, \ldots, n-1$. Their corresponding probability density functions ( $p d f s$ ), cumulative density functions (cdfs) and survival functions ( $s f s$ ) are thus denoted as $f_{i}(t), F_{i}(t), S_{i}(t) . T_{i \rightarrow k}$ is a random variable denoting the sum of the times residing in states $i, i+1, \ldots, k-1$. Thus, $T_{i \rightarrow k}$ is the time it will take the process to go from state $i$ to state $k$. In addition, $f_{i \rightarrow k}(t), F_{i \rightarrow k}(t), S_{i \rightarrow k}(t)$ are the $p d f, c d f$ and $s f$ of $T_{i \rightarrow k}$, respectively.

If the asset state is in state 1 at time t, the conditional probability that it will transit to the next state in the next time step $\Delta t$ is given by:

$$
P\left(X_{t+1}=2 \mid X_{t}=1\right)=p_{1,2}(t)=\frac{f_{1}(t) \cdot \Delta t}{S_{1}(t)}
$$

when $t=0$, the process entered into state 1 , i.e. new asset. If the time step is assumed to be small enough to exclude a two-state deterioration, $\Delta \mathrm{t}$ can be omitted.

If the process is in state 2 at time $t$, the conditional probability that it will transit the next state in the next time step $\Delta t$ is given by:

$$
P\left(X_{t+1}=3 \mid X_{t}=2\right)=p_{2,3}(t)=\frac{f_{1 \rightarrow 2}(t)}{S_{1 \rightarrow 2}(t)-S_{1}(t)}
$$

Note that the pdf $f_{1 \rightarrow 2}(t)$ pertains to $\mathrm{T}_{\mathrm{i} \rightarrow \mathrm{k}}$, which is the random variable denoting the sum of duration times in states 1 and 2.

The survival functions $S_{1 \rightarrow 2}(t)$ and $S_{1}(t)$ express the simultaneous condition that $\mathrm{T}_{1 \rightarrow 2}<\mathrm{t}$ and $\mathrm{T}_{1}<\mathrm{t}$

The equation above can then be generalised for subsequent states by: 


$$
\begin{gathered}
P\left(X_{t+1}=i+1 \mid X_{t}=i\right)=p_{i, i+1}(t)=\frac{f_{1 \rightarrow i}(t)}{S_{1 \rightarrow i}(t)-S_{1 \rightarrow(i-1)}(t)} \\
i=[1,2, \ldots, n-1]
\end{gathered}
$$

The conditional probabilities in Equation (2.7) provides all the transition probabilities $\mathrm{p}_{\mathrm{i}, \mathrm{i}+1}(\mathrm{t})$ to populate the transition probability matrix for the semi-Markov process.

$$
P^{t, t+1}=\left[\begin{array}{ccccc}
P_{11}^{t, t+1} & P_{12}^{t, t+1} & 0 & \ldots & 0 \\
0 & P_{22}^{t, t+1} & P_{23}^{t, t+1} & \ldots & 0 \\
\ldots & \ldots & \ldots & \ldots & \ldots \\
0 & 0 & \ldots & P_{n-1, n-1}^{t, t+1} & P_{n-1, n}^{t, t+1} \\
0 & 0 & \ldots & 0 & P_{n, n}^{t, t+1}
\end{array}\right]
$$

These transition probabilities are then time-dependent. Once the transition probability matrix is established, the deterioration process is modelled using Equation (2.9), to obtain the future probability of the process being in any state at any time $t+k$.

$$
P(t+k)=P(t) \times P^{t, t+1} \times P^{t+1, t+2} \cdots P^{t+k-1, t+k}
$$

$\mathrm{Ng}$ and Moses (1998) proposed that the Markov assumptions can be relaxed by the use of the semi-Markov process where the distribution of the holding time is not necessary exponential. They also discussed the use of the condition rating and concluded that this is not adequate as a performance indicator. It does not reflect the structure integrity of a bridge nor the improvement needed. The paper then demonstrated a method of determining the distribution for the holding times. The probability distribution function of the holding time can be calculated by an integral convolution between two age distributions for respective states. The paper also demonstrated the method on bridge condition data recorded in Indiana between 1988 and 1991 and compare with the prediction of the normal Markov model produced by Jiang and Sinha (1990). It showed a better deterioration model compared with traditional Markov model. Though, the study was based on real bridge condition data, the condition data is for the whole bridge, not bridge elements.

Kleiner (2001) also presented an asset deterioration model based on the semi-Markov approach. The waiting time of the process in any state was modelled as a random variable with a two- 
parameter Weibull probability distribution. The application of the model is then demonstrated based on hypothetical data, which was obtained from expert opinion and perception. Having the Weibull distribution parameters defined by the experts, the transition probability matrix was then obtained and the future condition of the assets was predicted. The paper demonstrated a model framework based on semi-Markov process, however the study was based on expert judgement, not the real data.

Mishalani and Madanate (2002) presented a study using the semiMarkov approach based on empirical data. The data, in this case, being condition ratings taken from the Indiana Bridge Inventory (IBI). There are 10 states in the model with 9 being the best and 0 being the worst state. The data set contained 1,460 records from 1974 to 1984 with two year inspection periods (which means there are about 5 sets of inspection data per structure). Following the analysis, data which indicate maintenance were removed. The following assumptions were also used: the time when at which an event occurred (condition deterioration) is exactly in the middle of two recorded inspection i.e. the time when the change in the condition rating occurs is exactly halfway between the two inspections; when condition drops by two states, the time in the intermediate state is assumed to be 1 year. Due to the unavailability of the data, the prediction study was carried out to model the degradation process between only three states (state 8,7 , and 6 ). This means that the empirical study was incomplete and did not contribute to the model framework established previously by other authors.

Yang et al. (2009) discussed the limitations imposed by the nature of condition data. All the previous models do not consider the impacts from non-periodical inspection, they also do not consider the maintenance requirements for specific deteriorated states in the model for different types of bridge elements. The paper then proposed a state definition system based on different types of maintenance actions required as described in Table 2.4.

\begin{tabular}{|c|c|}
\hline Discrete state & Different types of maintenance actions \\
\hline State 1 & Do nothing \\
\hline State 2 & Preventative maintenance \\
\hline State 3 & Corrective maintenance \\
\hline State 4 & Minor rehabilitation \\
\hline State 5 & Major rehabilitation \\
\hline
\end{tabular}

Table 2.4: Proposed rating systems for elements undergoing non-periodical inspection (Yang et al., 2009) 
The proposed transition probability matrix for this system which integrates both deterioration rates and improvement rates of a bridge element undergoing non-periodical inspection would then be:

$$
P=\left[\begin{array}{ccccc}
P_{11} & P_{12} & P_{13} & P_{14} & P_{15} \\
P_{21} & P_{22} & 0 & 0 & 0 \\
P_{31} & 0 & P_{33} & 0 & 0 \\
1 & 0 & 0 & 0 & 0 \\
1 & 0 & 0 & 0 & 0
\end{array}\right]
$$

The transition probability matrix reflects the outcome of different types of maintenances. For example, the outcome of preventative maintenance is to remain in its present state if residing in state 1 or improve from its present state to state 1 if it resides in any other state. The paper also presented a numerical method to estimate the transition probabilities in the transition probability matrix. The proposed model was developed based on the limitation of the specific condition data available. Thus the paper only presented the model formulation, the application as well as verification of the model on real data were not reported.

Thomas and Sobanjo (2013) developed a semi-Markov approach which uses the deterioration models from the authors' previous paper (Sobanjo et al., 2010). The authors stated that previous models only consider simple degradation processes which allow only a single state degradation in one transition period. In the newly developed model, the model states can have a maximum drop of two condition states. The interval transition matrix is then given as:

$$
P(t)=\left[\begin{array}{ccccc}
p_{i j-1} H_{i j-1}(t) & p_{i j} H_{i j}(t) & p_{i j+1} H_{i j+1}(t) & \ldots & \ldots \\
0 & \ldots & \ldots & \ldots & \ldots \\
0 & 0 & \ldots & \ldots & \ldots \\
0 & 0 & 0 & \ldots & \ldots \\
0 & 0 & 0 & 0 & 1
\end{array}\right]
$$

where

$p_{i j}$ is the probability for the embedded Markov chain of the semi-Markov process.

$H_{i j}(t)$ is the cumulative distribution of the sojourn time between condition state $i$ to state $j$.

The distribution of the time remaining in a condition state was modelled using a Weibull distribution. The author suggested that, 
the transition probabilities $\left(H_{i j-1}, H_{i j}, H_{i j+1}\right)$ between states can be computed using three-step computations as:

where:

$$
\begin{aligned}
& H_{11}=S_{1}(t) \\
& H_{13}=\sum_{x=1}^{t} f_{12}(x)\left[\frac{F_{23}(t-x)-F_{32}(0)}{1-F_{23}(0)}\right] \\
& H_{12}=1-\left(P_{11}(t)+P_{13}(t)\right)
\end{aligned}
$$

$S_{i}(t)=e^{\left(\frac{-t}{\eta}\right)^{\beta}}$ is the survivor function for the sojourn time in state $\mathrm{i}$

$F_{i j}(t)=1-e^{\left(\frac{-t}{\eta}\right)^{\beta}}$ is the cumulative distribution of the sojourn time between condition state $i$ and condition state $j$ at time t. $\beta$ and $\eta$ are Weibull parameters.

$f_{i j}(x)$ is the probability density function describing the sojourn time before the transition at a time $x$, from state $i$ to state $j$.

$P_{i j}$ is the probability of the bridge element moving from state $i$ to state $j$.

The deterioration curves generated by the semi-Markov model are then compared with those produced by the Markov model and actual degradation data. The semi-Markov approach produced a closer match with the actual degradation profile. Even though the paper contained a comprehensive study, the method suggested in this paper suffers from several limitations such as:

- The three-step computations illustrated were only able to predict the bridge condition up to 20 years. The future condition converged before reaching the lowest possible condition (i.e. a component never fails and is replaced). This can be avoided using more step equations although this will add significantly to the computation time. (Sobanjo, 2011)

- It was assumed that $5 \%$ of transitions cause a drop of two ratings so $\mathrm{p}_{\mathrm{ij}-1}=1 ; \mathrm{p}_{\mathrm{ij}}=1-0.05=0.95 ; \mathrm{p}_{\mathrm{ij}+1}=0.05$ (Equation (2.11)). This assumption is not justified, and will lead to inaccuracy.

- The bridge condition data is not ideally suitable to calculate the sojourn times (the times staying in a condition). 


\subsection{Summary and discussion}

It was shown that a reasonable amount of research has been carried out to establish reliable bridge deterioration models over the last three decades. Markov, semi-Markov and reliability-based approaches have previously developed for this purpose. The majority of deterioration models have adapted Markov chain process in predicting the deterioration process and future condition of a bridge or a bridge element. There are also a number of studies based on semi-Markov and reliability-based approaches, however these studies often lack application and verification with real data. All of these approaches are able to capture the stochastic nature of the deterioration process. Thus, these models predict the future asset condition in terms of the probability of being in each of the potential states.

Overall, Markov deterioration models have proved to be the most popular in modelling the bridge asset deterioration process. This is because the Markov approach is relatively simple to allow a fast and adequate study using bridge condition data. The model accounts for the present condition in predicting the future condition. However, the reviewed models are simple models which were developed for either one component or for an individual bridge, not for a bridge system that consist of many different components. Moreover, the Markov approach suffers from some limitations such as:

- Constant deterioration rates,

- The model size increases exponentially with the increasing number of states (or number of modelled components),

- The estimation of the transition probability using regression method is seriously affected by any prior maintenance actions (i.e. a rise in condition score) (Ortiz-García et al., 2006),

- The estimation of transition probabilities using the frequency approach requires: at least two consecutive condition records without any interventions for a large number of bridge components at different condition states, in order to generate reliable transition probabilities (Agrawal and Kawaguchi, 2009),

- The effects of maintenance is not captured i.e. the degradation process is treated the same before and after intervention. 
In contrast to the Markov model, a semi-Markov model often uses a Weibull distribution to model the time residing in the different states. Models based on semi-Markov approach are then capable of using non-constant deterioration rates which overcomes a major limitation. Though, the approach is based on the Markovchain process and still suffers from some similar disadvantages as in traditional Markov models:

- The model size increases exponentially with the increasing number of states (or number of modelled components),

- The estimation of transition probabilities requires complicated numerical solutions with associated computation time,

- The effects of maintenance is not captured i.e. the degradation process is treated the same before and after intervention.

In reliability-based models, the degradation process of bridges or bridge elements is modelled based on the life time analysis technique. An appropriate distribution is selected to model the times of a bridge component reaching a specified condition state. This approach considers both complete and incomplete lifetime data. It was demonstrated in all the review studies that the Weibull distribution is a good fit to these life time data. Also the obtained distribution parameters obtained indicate a non-constant i.e. increasing deterioration rates of bridge elements. Although the method is robust to model the degradation process between different states, a complete deterioration model comprising of all component states have not been developed.

All approaches discussed are based on statistical analysis of condition ratings, however, it is believed that the condition data used is inadequate for any detailed study of the degradation characteristic. There are serious limitations associated which affect the prediction results such as:

- Condition data is based on the subjective evaluation by bridge inspectors with the reliability of the ratings dependent on the experience of the inspectors (Office of Rail Regulation, 2007). Moreover, condition rating does not reflect the structural integrity of a bridge,

- Constant periods between inspections means that the data is collected at fixed intervals, thus the time at which a 
change in state of the bridge component is experienced is unknown and is often assumed to occur half way between inspection interval, this introduces bias variables in the times to these events thus affects the distribution fitted to these times,

- Condition data does not reflect the effects of maintenance. Data indicates rises in the score are usually removed. This means that the effect of maintenance is often ignored. 


\section{Chapter 3 - Data Analysis and Deterioration Modelling}

\subsection{Introduction}

The research aim is to study the real data to understand the structure characteristics and the deterioration process. This real data used in the study was provided by Network Rail, who owns and operates most of the UK railway infrastructures. It contains historical maintenance records of the bridge elements, including the inspection dates and the component condition scores. As discussed in Chapter 2, the bridge condition score system is believed to be inadequate to provide a sound study of the bridge element deterioration process. This chapter presents a novel method of modelling the asset deterioration process, this involves constructing a timeline of all historical work done of a bridge element and analysing the life time of the component reaching these intervention actions. The analysis methodology will be presented in detail after the discussion of the available datasets. Finally, the chapter presents the results obtained from the analysis.

\subsection{Database}

There are the five datasets that are used in this research. Table 3.1 shows the size of these datasets and their description as well as the information that was extracted for the study.

The CARRS dataset contains asset information on the structure and the work done reports. The dataset was developed in 2007 to replace multiple local systems currently in operation throughout 
the railway network (i.e. local databases and spreadsheets) and to have a single asset management system containing information of the whole structures asset portfolio. The oldest record in the CARRS dataset dates back to 1994 until current date.

The VERA dataset contains structure assessment reports from 1950 up to 2010. These reports contain structural assessments in term of loading capability, maximum stress, and suggestions whether if strengthening is required or if the bridge is capable for running a certain train speed. However only about $37 \%(12,628$ records) of the dataset contains some information, the rest are blank records.

The SCMI dataset is the biggest database on the bridge structures and contains very useful information about the minor and major elements of individual bridge asset. This system was designed by Network Rail as a high level asset management tool to measure and demonstrate the change in condition of bridge stock with time. Bridge components were inspected and rated with a score between $0-100$. There are two key issues that were reported with this system, they are:

- First is the low rate of structures examination. The company' standards require a detailed condition survey of each bridge at a normal interval of 6 years, with the system starting in 2000, there is only $60 \%$ of the bridges were inspected by 2006-2007. The company is well behind the inspection programme and this is reflected in this dataset as most of the structures only contain one set of scores over the course of 10 years period starting from 2000 up to current date (Network Rail, 2007a).

- Second is the concern expressed, by the Office of Rail Regulation (ORR), about the accuracy of the score recorded (Office of Rail Regulation, 2007).

Both of the CAF and MONITOR datasets contains intervention records with associated costs (expected cost and actual cost) on bridge asset. The CAF dataset collects information for major interventions, typically those with an expenditure of over $£ 50,000$. The MONITOR dataset collects information for smaller interventions with the associated cost of less than $£ 50,000$. 


\begin{tabular}{|c|c|c|c|}
\hline Dataset & $\begin{array}{l}\text { Number } \\
\text { of } \\
\text { records }\end{array}$ & Description & Extracted information \\
\hline $\begin{array}{l}\text { CARRS } \\
\text { (Civil Asset } \\
\text { Register and } \\
\text { Electronic } \\
\text { Reporting } \\
\text { System) }\end{array}$ & 20,312 & $\begin{array}{l}\text { Contains structure asset } \\
\text { information and work } \\
\text { done reports. CARRS was } \\
\text { developed as a structure } \\
\text { asset management } \\
\text { system to operate at } \\
\text { national level. }\end{array}$ & $\begin{array}{l}\text {-Brief scope of repair work } \\
\text {-Type of work done } \\
\text {-Implementation date }\end{array}$ \\
\hline $\begin{array}{l}\text { VERA } \\
\text { (Structures } \\
\text { Assessment } \\
\text { Database) } \\
\end{array}$ & 33,588 & $\begin{array}{l}\text { Contains information of } \\
\text { structure assessment } \\
\text { including reports on any } \\
\text { critical part of a structure. }\end{array}$ & $\begin{array}{l}\text {-Date when the structure } \\
\text { were inspected or assessed } \\
\text {-General notes about } \\
\text { inspected bridge elements }\end{array}$ \\
\hline $\begin{array}{l}\text { SCMI } \\
\text { (Structure } \\
\text { Condition } \\
\text { Monitoring } \\
\text { Index) }\end{array}$ & 871,211 & $\begin{array}{l}\text { Contains } 30,000 \text { bridge } \\
\text { assets and is used as part } \\
\text { of a risk assessment to } \\
\text { set detailed examination } \\
\text { frequencies and the } \\
\text { component scores } \\
\text { highlight areas of concern } \\
\text { that can be addressed. } \\
\text { The SCMI database has } \\
\text { also been extensively } \\
\text { used to identify structures } \\
\text { with particular generic } \\
\text { features, this enable the } \\
\text { managing risk on the } \\
\text { network-wide basis. }\end{array}$ & $\begin{array}{l}\text {-Bridge type } \\
\text {-List of bridge elements and } \\
\text { its materials } \\
\text {-Date when the structure } \\
\text { were inspected }\end{array}$ \\
\hline $\begin{array}{l}\text { CAF } \\
\text { (Cost Analysis } \\
\text { Framework) }\end{array}$ & 1,048 & $\begin{array}{l}\text { Contains information } \\
\text { about major repeatable } \\
\text { work activities for which } \\
\text { the meaningful volumes } \\
\text { can be defined. This is to } \\
\text { help NR to study the cost } \\
\text { and maintenance } \\
\text { expenditure. }\end{array}$ & $\begin{array}{l}\text {-Brief scope of repair work } \\
\text {-Type of work done } \\
\text {-Implementation date }\end{array}$ \\
\hline MONITOR & 32,359 & 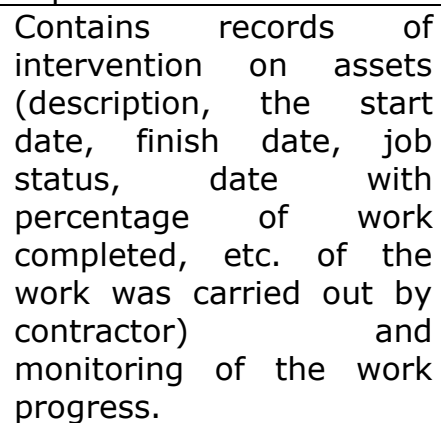 & $\begin{array}{l}\text {-Work location on structure } \\
\text {-Brief scope of repair work } \\
\text {-Actual work done } \\
\text {-Contractor work done notes } \\
\text {-Start date of repair works } \\
\text {-Finish date of repair works }\end{array}$ \\
\hline
\end{tabular}

Table 3.1: Datasets overview

Prior to the data analysis, all these datasets must be merged together to form a single working database that contains necessary information for the study. The first two steps were to combine and to cleanse all these different datasets. The third step was to filter and query only relevant data for each bridge substructures. Details of these steps are explained in Appendix A-1. Even though, the datasets are sparse and poorly structured, effort was made to ensure the data are merged and extracted sensibly. 
The final working dataset then contains information about each individual asset. It contains not only the structure information, but also the details of the works that have been done, associated costs, previous inspection, and any other work related records. All of these information fields in the working database are illustrated in Figure 3.1. It is worth mentioning that with the issues associated with the SCMI score, this data was not studied in this research. The research focuses mainly on historical maintenance data to study the degradation process of an asset.

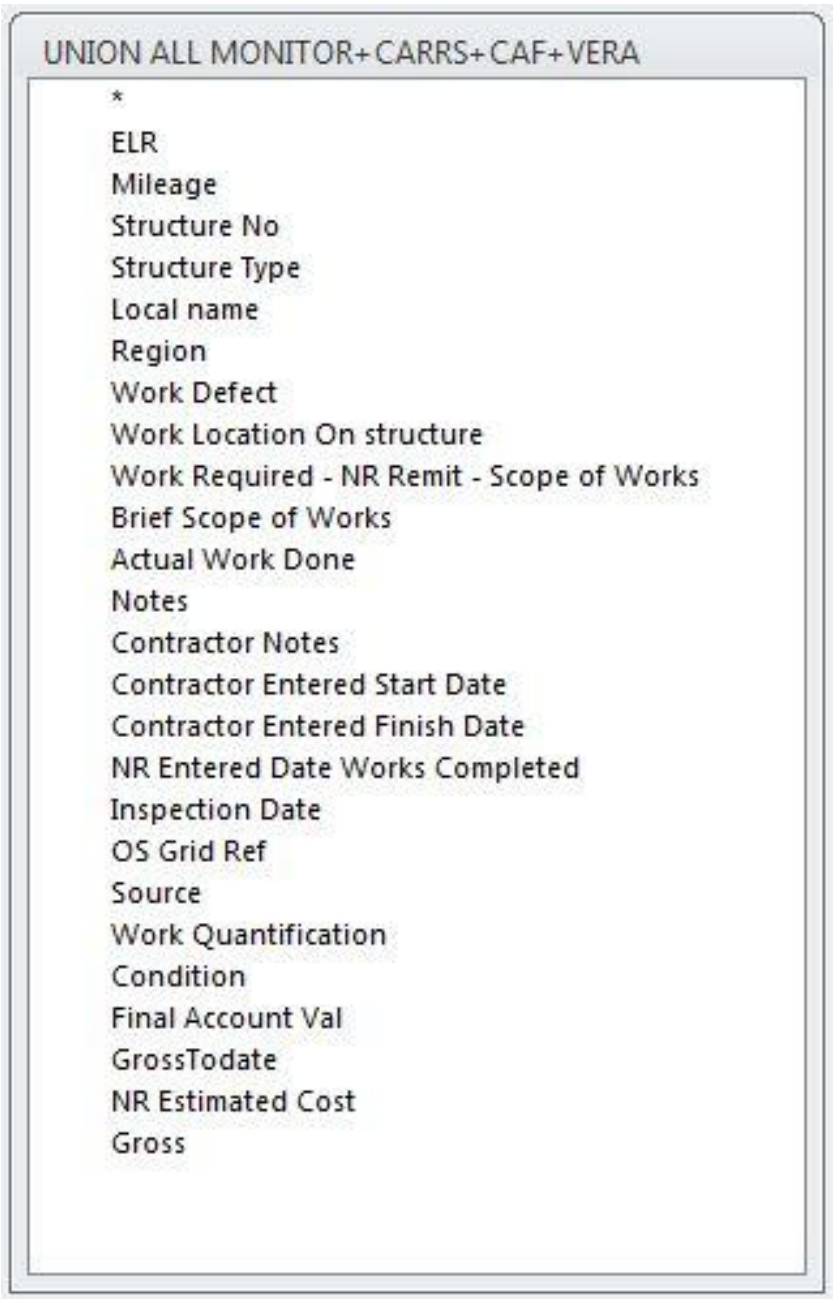

Figure 3.1: Information fields in a single working database after the merging and cleansing of all different datasets

\subsubsection{Data problems and assumptions}

- Large amount of data was not recorded in descriptive words but rather sentences or paragraphs, information was extracted from these data by reading through each record manually. 
- Data with missing or misleading information were not studied (blank date, work description missing, date records as database defaults date $01 / 01 / 1900$, repair work is marked as 'cancelled work').

- Data with different format and minor typos was corrected and used in the study. Bridge location stored in chain unit, instead of mileages, were converted to ensure the compatibility across all the databases. Records are believe to be minor typos were fixed e.g. year recorded as 3008 was changed to 2008.

- Most of the recorded works on metal bridge girders indicate the work was done on a set of girders. It is difficult then to analyse the work done on a single component. Assuming the girders behave in the same way, an estimation method was derived to allow a study on a single component to be carried out. This is discussed in more detail later on, in section 3.5.3.

- A single record in the database sometimes contains history of several work, the cost associated with this record therefore is likely to be a total cost of all the works mentioned. These cost figures are ignored when calculating the average costs for one specific work type.

\subsubsection{Bridge types}

Bridges under Network Rail management are classified into underbridges and overbridge. Each type of the bridge is further categorised into their main material: masonry, concrete, metal and other (timber, composite, etc.). It is worth noting that, the focus of this research is on the studying of the metal underbridges asset group. The reason for this is, upon the examination of the database, the data available to support the study for this asset group is more than other types of bridges. Moreover, metallic bridges deteriorate faster when comparing with concrete and masonry bridges making them one of the most critical asset groups.

\subsubsection{Bridge major elements}

The bridge is a complex structure, typical bridge elements for metal underbridges are illustrated in Appendix A-2. However, the data is only available for four main bridge components to be studied which are bridge deck, girder, bearing and abutment 
(Figure 3.2). These components are also studied according to different material types (metal, concrete, masonry, timber).

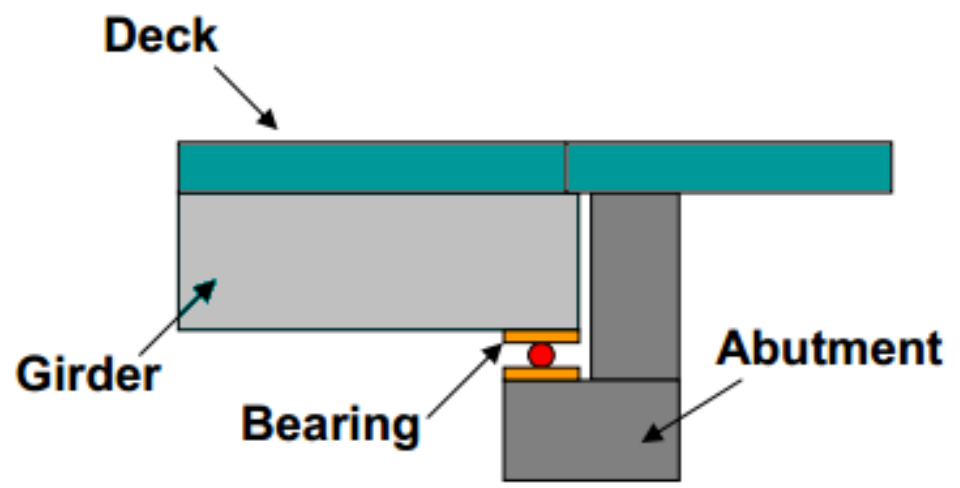

Figure 3.2: Bridge components studied

\subsection{Element condition states}

In order to study the degradation process of a bridge element, the states in which the component resides in throughout its entire life should be defined. In this research, the condition of a component is defined based on the level of defects. It was adopted that there are four condition states that a bridge component can be in: 'as new', good, poor and very poor state. These states are explained in Table 3.2 below for each type of component material. The states were deduced by studying both severity and extent (as defined by Network Rail's standard (Network Rail, 2004b)) of a defect on a particular bridge element. Details of the study as well as the definitions of all levels of degradations are explained in Appendix A-3.

\begin{tabular}{|c|c|c|c|c|}
\hline & \multicolumn{4}{|c|}{ Level of degradations } \\
\hline & Metal & Concrete & Timber & Masonry \\
\hline New & $\begin{array}{l}\text { Minor or no } \\
\text { defects }\end{array}$ & Minor or no defects & $\begin{array}{l}\text { Minor or no } \\
\text { defects }\end{array}$ & $\begin{array}{l}\text { Minor or no } \\
\text { defects }\end{array}$ \\
\hline $\begin{array}{l}\text { Good } \\
\text { State }\end{array}$ & Minor corrosion & $\begin{array}{l}\text { Spalling, small } \\
\text { cracks, exposed of } \\
\text { secondary } \\
\text { reinforcement }\end{array}$ & $\begin{array}{l}\text { Surface softening, } \\
\text { splits }\end{array}$ & $\begin{array}{l}\text { Spalling, } \\
\text { pointing } \\
\text { degradation, } \\
\text { water ingress }\end{array}$ \\
\hline $\begin{array}{l}\text { Poor } \\
\text { State }\end{array}$ & $\begin{array}{l}\text { Major corrosion, } \\
\text { loss of section, } \\
\text { fracture, crack } \\
\text { welds }\end{array}$ & $\begin{array}{l}\text { Exposed of primary } \\
\text { reinforcement }\end{array}$ & $\begin{array}{l}\text { Surface and } \\
\text { internal softening, } \\
\text { crushing, loss of } \\
\text { timber section }\end{array}$ & $\begin{array}{l}\text { Spalling, } \\
\text { hollowness, } \\
\text { drumming }\end{array}$ \\
\hline $\begin{array}{l}\text { Very } \\
\text { Poor } \\
\text { State }\end{array}$ & $\begin{array}{l}\text { Major loss of } \\
\text { section, } \\
\text { buckling, } \\
\text { permanent } \\
\text { distortion }\end{array}$ & $\begin{array}{l}\text { Permanent } \\
\text { structural damage }\end{array}$ & $\begin{array}{l}\text { Permanent } \\
\text { structural damage }\end{array}$ & $\begin{array}{l}\text { Missing } \\
\text { masonry, } \\
\text { permanent } \\
\text { distortion }\end{array}$ \\
\hline
\end{tabular}

Table 3.2: Condition states of a bridge element based on levels of degradations 


\subsection{Interventions}

It can be seen in the literature that, generally, maintenance actions are often categorised into two (Hearn et al., 2010, Yang et al., 2006, Frangopol et al., 2006) or four (Yang et al., 2009) maintenance categories. Some systems divided the maintenance type according to the nature of the work, others classified according to the frequency of the work carried out. The database studied in this research uses the following work categories: preventative (protection, painting, water-proofing); minor works; major works; strengthening; replacement. Based on element state condition as previously defined and the work description as given in the database, four maintenance categories were adopted and their definitions are given in the Table 3.3.

\begin{tabular}{|c|c|c|c|c|}
\hline Maintenance & \multicolumn{4}{|c|}{ Definition } \\
\hline \multirow{3}{*}{ Minor repair } & \multicolumn{4}{|c|}{$\begin{array}{l}\text { Minor repair implies the restoration of the structure element from the } \\
\text { good condition to the as new condition. Components in the good } \\
\text { condition can experience the following defects }\end{array}$} \\
\hline & Metal & Concrete & Timber & Masonry \\
\hline & $\begin{array}{l}\text { Minor } \\
\text { corrosion }\end{array}$ & $\begin{array}{l}\text { Spalling, small } \\
\text { cracks, exposed } \\
\text { of secondary } \\
\text { reinforcement }\end{array}$ & $\begin{array}{l}\text { Surface } \\
\text { softening, splits }\end{array}$ & $\begin{array}{l}\text { Spalling, } \\
\text { pointing } \\
\text { degradation } \\
\text { water ingress }\end{array}$ \\
\hline \multirow[b]{3}{*}{ Major repair } & \multicolumn{4}{|c|}{$\begin{array}{l}\text { Major repair implies the restoration of the structure element from the } \\
\text { poor condition to the as new condition. Components in the poor } \\
\text { condition can experience the following defects }\end{array}$} \\
\hline & Metal & Concrete & Timber & Masonry \\
\hline & $\begin{array}{l}\text { Major } \\
\text { corrosion, loss } \\
\text { of section, } \\
\text { fracture, } \\
\text { cracked welds }\end{array}$ & $\begin{array}{l}\text { Exposed of } \\
\text { primary } \\
\text { reinforcement }\end{array}$ & $\begin{array}{l}\text { Surface and } \\
\text { internal } \\
\text { softening, } \\
\text { crushing, loss of } \\
\text { timber section } \\
\end{array}$ & $\begin{array}{l}\text { Spalling, } \\
\text { hollowness, } \\
\text { drumming }\end{array}$ \\
\hline \multirow[b]{3}{*}{ Replacement } & \multicolumn{4}{|c|}{$\begin{array}{l}\text { Complete replacement of a component or the whole bridge. } \\
\text { Components in the very poor condition can experience the following } \\
\text { defects }\end{array}$} \\
\hline & Metal & Concrete & Timber & Masonry \\
\hline & $\begin{array}{l}\text { Major loss of } \\
\text { section, } \\
\text { buckling, } \\
\text { permanent } \\
\text { distortion }\end{array}$ & $\begin{array}{l}\text { Permanent } \\
\text { structural } \\
\text { damage }\end{array}$ & $\begin{array}{l}\text { Permanent } \\
\text { structural } \\
\text { damage }\end{array}$ & $\begin{array}{l}\text { Missing } \\
\text { masonry, } \\
\text { permanent } \\
\text { Distortion }\end{array}$ \\
\hline Servicing & \multicolumn{4}{|c|}{$\begin{array}{l}\text { Activities that protect the structure from the source that drives the } \\
\text { degradation process. }\end{array}$} \\
\hline
\end{tabular}

Table 3.3: Maintenance types definitions

Servicing is the only type of maintenance which does not change the state of the component, servicing will slow down the degradation rate. Strengthening work is considered as a major repair. Minor repair, major repair and replacement are assumed to restore the component to the 'as good as new' condition. These three interventions can be carried out when the component 
reaches the good, poor or very poor state from the 'as new' condition.

\subsection{Deterioration modelling}

It is important to understand the asset and its component characteristics in order to develop an accurate asset model. Different components would experience different level of degradation and it is desirable to study components in a group which they share common factors that would cause similar degradation process. In other words, the study should be carried out on a group of components that have the same structure, material, environment, route criticality, traffic tonnage. Unfortunately, the information available were not enough for such detailed study, hence the components are grouped in term of structure and material for the degradation analysis.

\subsubsection{Life time data}

The degradation of a bridge element is analysed by studying the historical maintenance records throughout its lifetime. Typical deterioration pattern can be seen in Figure 3.3. The time to reach state $\mathrm{j}$ and $\mathrm{k}$ from new (state $\mathrm{i}$ ) are given as $T_{i, j}^{L}$ and $T_{i, k}^{L}$. These times are often called the time to failure and the term 'failure' used here does not mean the physical failure of a component but indicates the time to the point when a certain type of repair is necessary. It is important when analysing the lifetime data of a component to account for both complete data, $T_{i, k}^{L}$ and censored data, $T_{i, k}^{C}$. Complete data indicates the time of reaching state $\mathrm{k}$ from the new condition. Censored data is incomplete data where it has not been possible to measure the full lifetime. This may be because the component was repaired or replaced, for some reason, prior to reaching the condition $k$ and so the full life has not been observed. The components life is however known to be at least $T_{i, k}^{C}$. Figure 3.3 shows how the complete and censored time were being analysed. The time between major repair and minor repair is a complete time indicates the full life time of the component reaching the state where minor repair is required from the 'as new' state. This time is also the censored time indicates the component's condition was restored to new condition before it reaches the state where major repair is necessary hence the full time between two major repairs cannot be measured. In the case 
where bridge strike happened, the time between the last repair and the time when the accident happened is censored time since the repair responded to the accident rather than the degradation of the component.

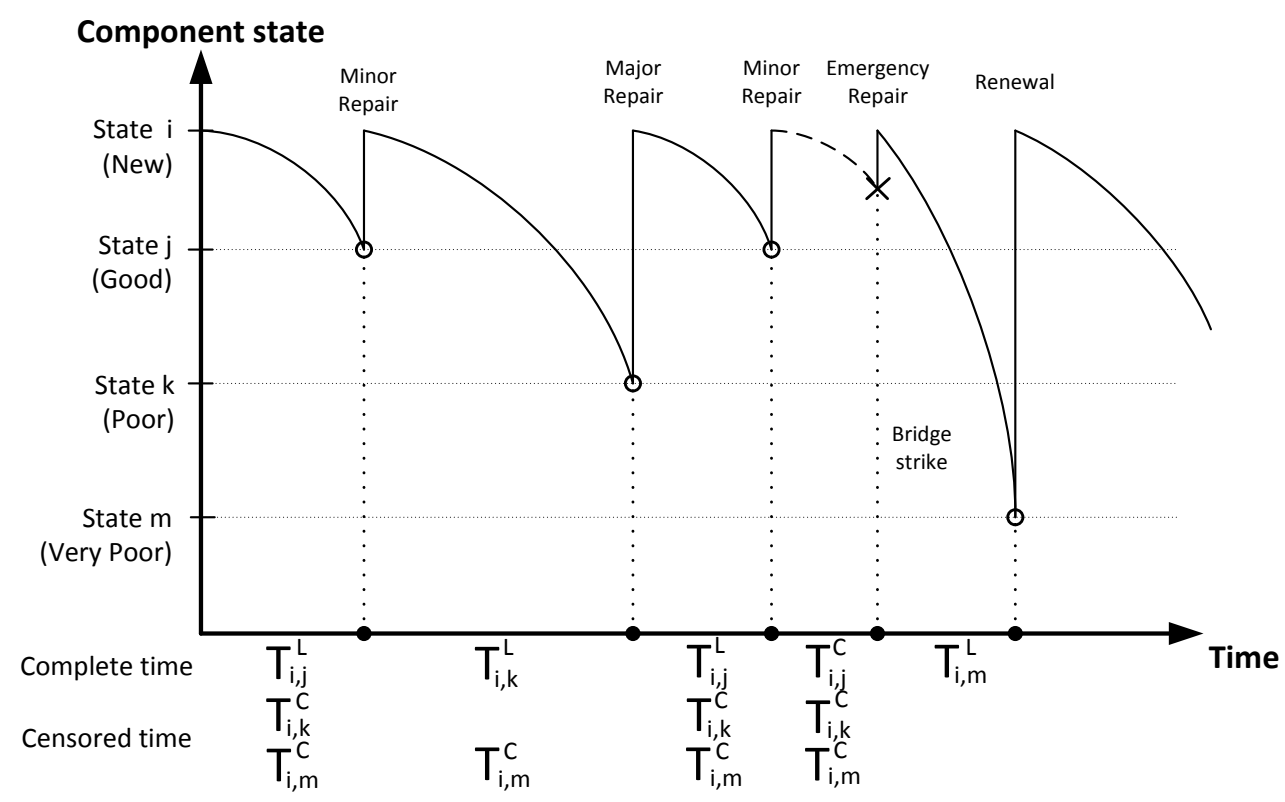

Figure 3.3: Typical deterioration pattern and historical work done on a bridge component

\subsubsection{Distribution fitting}

Having obtaining the lifetime data for bridge components, components of the same type and materials can be grouped together where the data is fitted with a distribution. A range of distributions are used (Weibull, Lognormal, Exponential, Normal). The goodness-of-fit test is used to compare the fitness of these distributions. The test involves visual observation of the probability plot and the conduction of a statistic hypothesis test (Anderson-Darling test (Stephens, 2012)). The two-parameter Weibull distributions were found to be the best fitted distribution in most of the cases, this agrees with the fact that Weibull is well known for its versatility to fit the life time data, and is a commonly used distribution in life data reliability analysis. For the two-parameter Weibull distribution, the general expression for the probability density function is:

$$
f(t)=\frac{\beta}{\eta}\left(\frac{t}{\eta}\right)^{\beta-1} e^{-\left(\frac{t}{\eta}\right)^{\beta}}
$$

$\beta$ is the shape parameter

$\eta$ is the scale parameter 
The Weibull distribution's parameters are determined using the rank regression method. With the shape and scale parameter of the Weibull distribution derived, we now have a distribution that statistically models the degradation process of a bridge element in terms of the times it takes to degrade from the 'as new' state to different condition states.

\subsubsection{Estimation method}

The disadvantage when studying lifetime data is that it requires a significant amount of data to allow a distribution to be fitted for accurate modelling. The nature of a bridge structure operating for long period of time sometimes results in a very few or no repair data. In the cases where the data were neither available nor enough to allow a distribution to be fitted, a simple estimation can be used to estimate the failure rates of a bridge component. The rate of reaching an intervention type is the total numbers of repairs on that group of components divided by the total time those components operate in (as given in Equation (3.2)). Assuming the failure rate is constant, the mean time to failure (MTTF) can be calculated simply as the inverse of the failure rate.

$$
\lambda_{i}=\frac{\sum_{1}^{n} N_{i}}{\sum_{1}^{n}\left[T_{i, j}^{L}+T_{i, j}^{C}\right]}
$$

where

$\mathrm{N}$ is the number of repairs on a single component

$\mathrm{n}$ is the number of components of type $i$ studied

$T_{i, j}^{L}$ is complete life-time of the component reaching

state $j$ from new (state $i$ )

$T_{i, j}^{C}$ is censored life-time of the component reaching state $j$ from new (state $i$ )

The nature of sparse data means that there are cases where there is a record indicating a repair has happened but there were no inspection before or after a repair. In this case, the time when the bridge was built was used to calculate the censored lifetime data i.e. the time between the repair and when the bridge was built is the operation time of the component and the failure rate is calculated by the quotient of one failure and this operation time. 


\subsubsection{Expert estimation}

In the case where no data is available at all, the degradation rates were estimated by consulting with a group of bridge engineering experts. It is worth noting that not only the degradation study requires experts' estimations but also other part of the project would require expert inputs such as when it comes to model assumptions or non-quantified effects of servicing and environment. This will be discussed in more detailed throughout the thesis when the expert estimation is required. The summaries of all the inputs from experts are:

- The replacement rates of bridge bearings and abutments,

- How much the environment affects the deterioration rate of bridge component,

- The degradation rates of metal coatings and the effects on the deterioration process of metal element,

- The repair scheduling times,

- The servicing cost of bridge decking and the renewal costs of bearings and abutments.

\subsubsection{Single component degradation rate estimation}

As mentioned earlier, the available historical data used in the study did not provide enough information to identify a particular element that maintenance action was performed on. This is the case of a bridge comprises of many girders, a historical record indicated an action was performed on one of these girders but it is not possible to know which one. When applying the method described above to the data, the degradation rates obtained would be for the group of girders. It is therefore required to estimate the degradation rate for a single girder.

Assuming each of the girders behaving in the same way i.e. they have the same degradation characteristic, and the times that girder 1 and 2 degrade to the degraded states are governed by Weibull distribution $\left(\beta_{2}, \eta_{2}\right)$. It is required to estimate the values of $\left(\beta_{2}, \eta_{2}\right)$ given that the values of $\left(\beta_{1}, \eta_{1}\right)$ are known from the study in the previous section. 


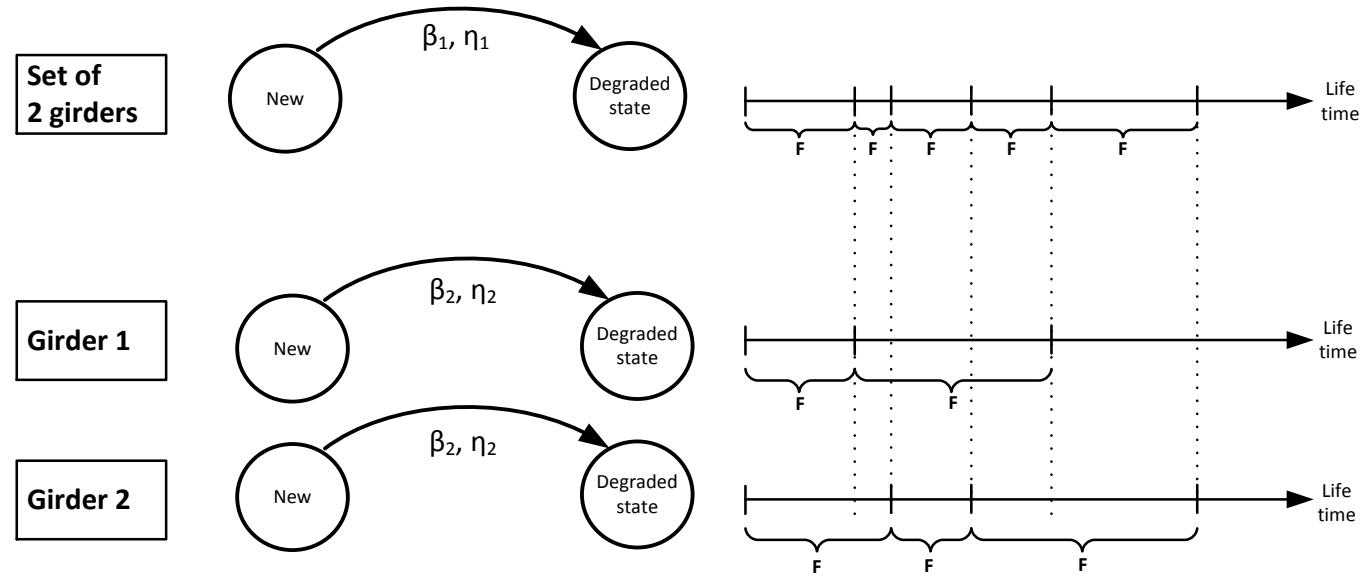

Figure 3.4: Single component degradation rate

Distributions of times for girder 1 and girder 2 to reach the degraded state from the new state can be generated as demonstrated in the time line shown in Figure 3.4. By combining these times and fitting a distribution, it is expected to obtain a distribution with the parameters very close to $\left(\beta_{1}, \eta_{1}\right)$. Thus an exhaustive search can be carried out to find the appropriate Weibull distribution $\left(\beta_{2}, \eta_{2}\right)$. The sequence of the search is described below:

1. For a range of $\left(\beta_{2}, \eta_{2}\right)$ values, complete life times for girder 1 and girder 2 are sampled. The life time is sampled until a certain simulation time is reached and the process is repeated for a number of generations.

2. The life times for girder 1 and girder 2 are combined together and then a Weibull distribution is fitted to the data where the parameters $\left(\beta_{1}^{\prime}, \eta_{1}^{\prime}\right)$ are obtained.

3. The most appropriate $\left(\beta_{2}, \eta_{2}\right)$ values is selected to produce $\left(\beta_{1}^{\prime}, \eta_{1}^{\prime}\right)$ so that $\left(\beta_{1}^{\prime}-\beta_{1}\right)$ AND $\left(\eta_{1}-\eta_{1}^{\prime}\right)$ are minimised.

\subsection{Results and Discussions}

\subsubsection{Component degradation analysis}

Table 3.4 shows the distribution parameters obtained after fitting a Weibull distribution to the life time data. The table also shows the cases where the data was not available for a distribution to be fitted statistically. In these cases, the estimation method was used to estimate the mean time to failure (MTTF) first before estimating the Weibull parameters. Since this method assumed a 
constant failure rate, the beta value of the Weibull distribution is set to one and the eta value is the same as the mean. This is statistically correct as exponential distribution is a special case of Weibull distribution when beta value equals to one and the eta value is the mean. The table also includes the distribution parameters estimated for a single main girder. This is because the data was only available for a set of main girders to be studied hence the distribution of the failure times for a single girder needs to be estimated.

The detail component degradation analysis for all of the bridge elements listed in the table can be found in Appendix A-4. Also presented in the appendix are the distribution of the component current condition; and the distribution of the specific repair in each work categories (minor, major repair, replacement, servicing).

\begin{tabular}{|c|c|c|c|c|c|c|c|c|}
\hline \multicolumn{7}{|c|}{ Weibull Fitting (Weibull 2-parameter) } & \multicolumn{2}{|c|}{ Number of data } \\
\hline $\begin{array}{l}\text { Bridge } \\
\text { component }\end{array}$ & Material & Condition & Intervention & Beta & $\begin{array}{c}\text { Eta } \\
\text { (year) }\end{array}$ & $\begin{array}{l}\text { Mean } \\
\text { (year) }\end{array}$ & Complete & Censored \\
\hline \multirow{3}{*}{$\begin{array}{l}\text { GIRDER (set } \\
\text { of two) }\end{array}$} & \multirow{3}{*}{ Metal } & Good & Minor Repair & 1.257 & 12.50 & 11.63 & 37 & 72 \\
\hline & & Poor & Major Repair & 0.801 & 27.91 & 31.58 & 12 & 35 \\
\hline & & Very Poor* & Replacement* & 1.000 & 116.84 & 116.84 & 3 & 1 \\
\hline \multirow{3}{*}{$\begin{array}{l}\text { GIRDER } \\
\text { (single) }\end{array}$} & \multirow{3}{*}{ Metal } & Good & Minor Repair & 1.71 & 23.39 & 20.86 & - & - \\
\hline & & Poor & Major Repair & 0.87 & 44.27 & 47.49 & - & - \\
\hline & & Very Poor & Replacement & 1.14 & 149.63 & 142.77 & - & - \\
\hline \multirow{9}{*}{ DECK } & \multirow{3}{*}{ Metal } & Good & Minor Repair & 1.265 & 10.28 & 9.54 & 16 & 67 \\
\hline & & Poor & Major Repair & 1.038 & 20.00 & 19.71 & 10 & 58 \\
\hline & & Very Poor & Replacement & 1.009 & 28.47 & 28.36 & 14 & 72 \\
\hline & \multirow{3}{*}{ Concrete } & Good & Minor Repair & 1.082 & 19.09 & 18.52 & 3 & 7 \\
\hline & & Poor* & Major Repair* & 1.000 & 26.67 & 26.67 & 0 & 4 \\
\hline & & Very Poor & Replacement & 0.976 & 34.26 & 34.63 & 2 & 10 \\
\hline & \multirow{3}{*}{ Timber } & Good & Minor Repair & 1.312 & 3.99 & 3.68 & 12 & 5 \\
\hline & & Poor & Major Repair & 1.371 & 7.13 & 6.52 & 5 & 6 \\
\hline & & Very Poor & Replacement & 1.501 & 6.12 & 5.52 & 27 & 40 \\
\hline \multirow{3}{*}{ BEARING } & \multirow{3}{*}{ Metal } & Good & Minor Repair & 0.838 & 14.94 & 16.41 & 12 & 39 \\
\hline & & Poor & Major Repair & 2.129 & 14.43 & 12.78 & 5 & 10 \\
\hline & & Very Poor* & Replacement* & 1.000 & 21.92 & 21.92 & 1 & 2 \\
\hline \multirow{3}{*}{ ABUTMENT } & \multirow{3}{*}{ Masonry } & Good* & Minor Repair* & 1.000 & 51.94 & 51.94 & 1 & 9 \\
\hline & & Poor* & Major Repair* & 1.000 & 100.87 & 100.87 & 1 & 2 \\
\hline & & Very Poor* & Replacement* & 1.000 & 150.00 & 150.00 & 0 & 0 \\
\hline
\end{tabular}

Table 3.4: Distribution parameters obtained from the life time study ('estimation method) 
There are a total of more than 37,000 bridge main girders component in the whole bridge population. The number of data contains historical work done are quite low, with only 604 sets of girders that actually contain useful records. This means that only $1.6 \%$ of the population were analysed. The Weibull shape parameter obtained illustrates that the rate of deteriorating from a new to a good condition for a main girder is increasing with time (wear-out characteristics). The failure rate is double after 20 years residing in new state. However the rate of reaching the poor condition shows unexpected behaviour, it is suspected that the lack of data has resulted in the decreasing rate of failure with time. Although there are a significant number of records on minor and major intervention, there are only 4 entries were recorded for the renewal of bridge main girders. Moreover, these records were missing inspection information which prevents the derivation of the lifetime data. Thus, a distribution could not be fitted to model the rate of main girders replacement. The estimation method was employed to estimate the rate of girder replacement.

For metal decks, the shape parameters obtains for a component reaching the poor and the very poor state are very close to one, this means that the rate of a component requires major repairs and replacement is almost constant over time and is about 0.05 and 0.03 metal deck per year. In contrast, the rate of metal decks moving from new condition to good condition is increasing from 0.06 metal decks per year to about 0.18 after 60 years. Thus it is three times more likely to require a minor repair for a metal deck in 60 years old comparing with the new metal deck.

Almost the entire population of concrete decks are in new and good conditions with only about $1 \%$ of the population is in the very poor condition that would need replacement. Also due to the young age of the concrete structure, not many historical data are available for the study, there were only 10 minor repairs, 4 major repairs and 12 deck replacements recorded.

Timber deck result demonstrated a very short live comparing with deckings of other materials. Also the failure rates for reaching different conditions increase significantly with time. The results show that the characteristic time for a timber deck to be replaced is actually faster than the time for it to undergo major repairs. This suggests that the deck would actually need to be replaced before it needs major repairs. The reason for this is because 
timber materials have a short life span, also timber is much harder to repair. Once the material reaches a point of severe defects, the timber deck is usually replaced. This preferable option of repairs is demonstrated by looking at the number of replacements recorded in the database. The number of replacements recorded in the database, more than 100 timber deck replacements, which is much greater than the number of times major repair were carried out (20 timber deck major repairs).

The rate at which the bearing would require a minor repair is almost constant at about 0.1 every year. Unexpectedly, it can be seen that the characteristic life of the bearing reaching a poor condition is actually shorter than that of reaching a good condition. The data that indicates a bearing major repair is often extracted from an entry that carries information about other repair works. Even though this entry is categorised in the database as major work, it might be that other works were major and the bearing repair might be opportunistic work. About $70 \%$ of bearing major repair data were extracted this way and since it is not possible to validate these entries, it is accepted that the data has influence these unexpected results.

The results obtained indicate that the abutment requires much less maintenance than other bridge elements with the mean time of an abutment to require minor repair is about 50 years. There were not enough data to allow the rate of abutment replacement to be calculated, which again agrees with the fact that abutment almost never require complete replacement, unless it is a complete demolition of the entire bridge due to upgrade or natural disaster.

In general, the distributions of times reaching different states for all the components suggested that, in most cases, we are expecting a slightly increasing deterioration rates over time. This is demonstrated by the beta value of the Weibull distribution is slightly larger than one.

\subsubsection{Servicing effects}

When there is no servicing, the deterioration rate is high, the rate decreases as the servicing interval decreases i.e. servicing more frequently, though the rate can only be reduced up to a certain level. To quantify the effects of the servicing on the deterioration 
rate of a particular component, a study can be carried out to look at the correlation between the degradation rates of similar component under different servicing intervals. For similar components with the same servicing time, they are grouped together and the average deterioration rate is determined (using Equation (3.2) in Section 3.5.3). If we do this again for other groups of components with different servicing times, the deterioration rates of these groups of components can be compared and the effect of servicing interval can then be investigated. Table 3.5 shows the estimated deterioration rates at different servicing interval for metal decking.

\begin{tabular}{|c|c|c|c|c|c|}
\hline \multicolumn{5}{|c|}{ Metal Decking } \\
\hline $\begin{array}{c}\text { Deterioration rate } \\
\text { from the 'as new' to a } \\
\text { good condition, } \boldsymbol{\lambda}_{\mathbf{1}}\end{array}$ & \multicolumn{2}{c|}{$\begin{array}{c}\text { Deterioration rate } \\
\text { from the 'as new' to a } \\
\text { poor condition, } \boldsymbol{\lambda}_{\mathbf{2}}\end{array}$} & $\begin{array}{c}\text { Deterioration rate } \\
\text { from the 'as new' to a } \\
\text { very poor condition, } \boldsymbol{\lambda}_{\mathbf{3}}\end{array}$ \\
\hline $\begin{array}{c}\text { Servicing } \\
\text { interval } \\
\text { (year) }\end{array}$ & $\begin{array}{c}\text { Deterioration } \\
\text { rate } \\
\text { (year }^{-1} \text { ) }\end{array}$ & $\begin{array}{c}\text { Servicing } \\
\text { interval } \\
\text { (year) }\end{array}$ & $\begin{array}{c}\text { Deterioration } \\
\text { rate } \\
\text { (year }^{-1} \text { ) }\end{array}$ & $\begin{array}{c}\text { Servicing } \\
\text { interval } \\
\text { (year) }\end{array}$ & $\begin{array}{c}\text { Deterioration } \\
\text { rate } \\
\left.\text { (year }{ }^{-1}\right)\end{array}$ \\
\hline 0.9 & 0.00848 & 0.9 & 0.00549 & 1.45 & 0.00462 \\
\hline 3.5 & 0.01591 & 3 & 0.01136 & 2.5 & 0.00734 \\
\hline 6 & 0.01697 & 10 & 0.01209 & 8 & 0.00768 \\
\hline 12.5 & 0.01756 & 14 & 0.01249 & 15 & 0.00787 \\
\hline
\end{tabular}

Table 3.5: Deterioration rates at different servicing frequency - Metal Decking

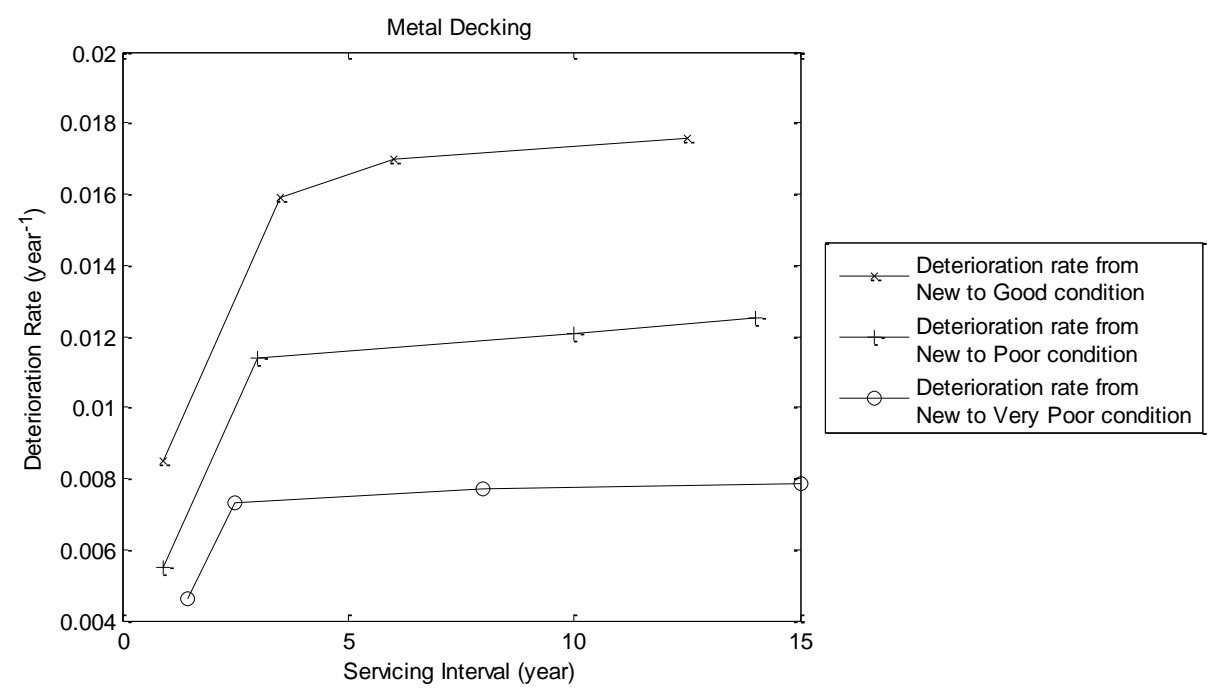

Figure 3.5: Effects of servicing intervals on deterioration rates - Metal Deck

In general, it follows that the deterioration rate increases as the servicing frequency decreases. For a metal deck that being serviced every 20 years instead of every year, the rate of deteriorating from the 'as new' to a good condition increases by a factor of two. Under these circumstances, the rate of an element 
moving to a poor condition and very poor condition increase by 2 and 1.53 times respectively.

Assuming a linear relationship between the servicing interval and the deterioration rate, an adjustment factor can be derived to reflect the effect of servicing on the deterioration rate of an element. Table 3.6 shows the adjustment factors for metal bridge decks at different servicing intervals. Note that the minimum and maximum interval considered in this research is 1 and 20 years respectively, this is also considered in practice (Network Rail, 2009). Similar investigations are studied on other components, however, except for the main girder, the data was insufficient to conduct the full study. These adjustment factors shown in Table 3.6 are then assumed to apply for other bridge components.

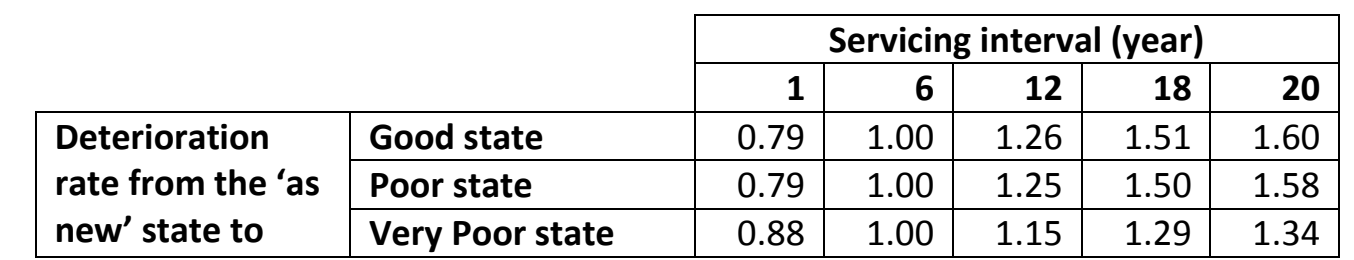

Table 3.6: Adjustment factors to the deterioration rate at different servicing interval for metal bridge deck

\subsubsection{Interventions}

Distribution of the intervention times is found by fitting a distribution to the duration of work for a certain type of intervention for a certain component. The duration of the work was taken as the duration between 'Contractor entered start date' and 'Contractor entered finished date' as recorded in the database. It is important to know that as the database does not always have a full record of all the start date and end date of a work, therefore when there is no available record for 'Contractor entered finished date', the 'NR (Network Rail) Entered Date Works Completed' was used instead. The results are presented in Table 3.7. 


\begin{tabular}{|c|c|c|c|c|c|}
\hline \multicolumn{6}{|c|}{ Weibull Fitting (2-parameter Weibull) } \\
\hline & & & Beta & Eta (day) & Mean duration (day) \\
\hline \multirow{4}{*}{ Girder } & \multirow{4}{*}{ Metal } & Servicing & 0.60 & 10.30 & 15.67 \\
\hline & & Minor Repair & 0.60 & 14.06 & 21.06 \\
\hline & & Major Repair & 0.89 & 34.98 & 37.11 \\
\hline & & Replacement* & 1.00 & 40.00 & 40.00 \\
\hline \multirow{12}{*}{ Deck } & \multirow{4}{*}{ Metal } & Servicing & 0.53 & 5.48 & 9.92 \\
\hline & & Minor Repair & 0.69 & 7.35 & 9.47 \\
\hline & & Major Repair & 0.57 & 31.60 & 50.47 \\
\hline & & Replacement & 0.66 & 43.35 & 57.81 \\
\hline & \multirow{4}{*}{ Concrete } & Servicing & 1.29 & 2.19 & 2.03 \\
\hline & & Minor Repair & 1.05 & 4.21 & 4.12 \\
\hline & & Major Repair & 0.53 & 17.64 & 31.50 \\
\hline & & Replacement & 0.67 & 28.74 & 38.15 \\
\hline & \multirow{4}{*}{ Timber } & Servicing & 0.53 & 6.28 & 11.37 \\
\hline & & Minor Repair & 0.66 & 6.98 & 9.35 \\
\hline & & Major Repair & 0.83 & 12.33 & 13.64 \\
\hline & & Replacement & 0.63 & 36.12 & 50.91 \\
\hline \multirow{4}{*}{ Bearing } & \multirow{4}{*}{ Metal } & Servicing & 0.70 & 10.49 & 13.35 \\
\hline & & Minor Repair & 0.53 & 12.56 & 22.52 \\
\hline & & Major Repair & 0.85 & 36.62 & 39.98 \\
\hline & & Replacement* & 1.00 & 50.00 & 50.00 \\
\hline \multirow{4}{*}{ Abutment } & \multirow{4}{*}{ Masonry } & Servicing & 0.65 & 5.54 & 7.60 \\
\hline & & Minor Repair & 0.75 & 8.27 & 9.86 \\
\hline & & Major Repair & 0.65 & 86.64 & 118.64 \\
\hline & & Replacement* & 1.00 & 150.00 & 150.00 \\
\hline
\end{tabular}

Table 3.7: Distribution of the repair times ( ${ }^{*}$ estimated rates using experts' opinions)

\subsubsection{Costs}

\section{Intervention costs}

Intervention costs were estimated from the database by calculating the average costs for each type of work and are shown in Table 3.8. Note that, one record in the database sometimes includes history of several works, this means that the cost would be the total cost of all the works, these figures were excluded from the study as the exact cost for one type of work cannot be determined. Also, the unit costs can sometimes be reported in $\mathrm{E} / \mathrm{m}^{2}$ unit, however information such as the area of work done and the area of a bridge component are not recorded in the database. Therefore, in this project, the average cost calculated is assumed as the cost per one intervention action. The cost is rounded to the nearest $£ 100$. 


\begin{tabular}{|c|c|c|c|c|c|c|}
\hline & & Decking & & Girder & Bearing & Abutment \\
\hline$(\mathbf{f})$ & Metal & Concrete & Timber & Metal & Metal & Timber \\
\hline Servicing & $1400^{*}$ & $1400^{*}$ & $1400^{*}$ & 6600 & 3600 & 3200 \\
\hline Minor Repair & 2700 & 2800 & 2800 & 6500 & 4400 & 5600 \\
\hline Major Repair & 6700 & 8100 & 7400 & 23900 & 22700 & 21300 \\
\hline Replacement & 22000 & 21000 & 21300 & 41600 & $40000^{*}$ & $80000^{*}$ \\
\hline
\end{tabular}

Table 3.8: Average intervention costs in $£$ (*expert estimation)

\section{Inspection costs}

The cost of inspection is a function of the asset criticality and the number of minor components that are going to be inspected and is shown in Table 3.9 (Network Rail, 2010b).

\begin{tabular}{|c|c|c|}
\hline \multirow{2}{*}{$\begin{array}{c}\text { Route } \\
\text { criticality } \\
\text { band }\end{array}$} & \multicolumn{2}{|c|}{ Inspection cost } \\
\cline { 2 - 3 } & Set up cost (£) & Cost per component (£) \\
\hline 1 & $£ 2,000$ & $£ 64$ \\
\hline 2 & $£ 500$ & $£ 64$ \\
\hline 3 & $£ 250$ & $£ 64$ \\
\hline 4 & $£ 250$ & $£ 64$ \\
\hline 5 & $£ 250$ & $£ 64$ \\
\hline
\end{tabular}

Table 3.9: Inspection costs (Network Rail, 2010b)

\subsection{Summary}

This chapter first discusses different datasets that are available for the bridge deterioration analysis. A data preparation process was carried out to merge and cleanse those datasets into a single working database where each asset is uniquely defined. This database contains information, up to elemental level, on the repair has been carried out, previous inspection, servicing, associated cost, etc. With the available data, the bridge components were studied are: metal girder, metal deck, concrete deck, timber deck, metal bearing, and masonry abutment. They are also considered as the major components that make up a metal underbridge. Based on the levels of defects for different bridge element materials, there are four states that the component can be in, they are: 'as new', good, poor, and very poor condition. From the 'as new' state, when to component reaches the good state, a minor intervention is necessary. And when the component is in the poor and very poor state, major repair and renewal is required respectively. The interventions also consider servicing, which is a type of work that does not change 
the state of the component but slows down the deterioration rates.

The chapter also presented a method of modelling the degradation of a bridge element by analysing its historical maintenance records. A life time of the component is calculated by the time the component takes to deteriorate from the 'as new' state to the state where an intervention was carried out. By gathering the lifetime for the component of the same type, a Weibull distribution is fitted to these data to statistically model the deterioration process. Where there is a little data available, the estimation method is used, and where there is no data available, estimation uses the experts' opinions. In the case where the degradation process was determined for a group of main girders, the simulation method of obtaining the distribution of lifetimes for a single girder was also described. The presented method demonstrates that 'life data' analysis method can be applied to model the deterioration process of bridge elements. This method recognises the 'censored' nature of bridge lifetime data and incorporates these data into the deterioration modelling process.

The distributions of the lifetimes for all bridge major components were obtained (Table 3.4). The results suggested that, for most of the cases, the deterioration rates of the components increase slightly over time. The study also confirmed the effect of the servicing on the slowing down of the deterioration rate, component is expect to deteriorate two times as fast when it is being serviced every 20 years comparing with one is serviced every year. The distributions of the repair times and the associated costs were also determined and the results were reported. All of these results would then be used later on in this research as the inputs for the bridge model.

Throughout the degradation study, several key problems and assumptions with the datasets were discussed. The availability as well as validity of the data has imposed a lot of constraints on the accurate modelling of the degradation rate with the proposed method. The degradation study also assumed 'perfect maintenance' i.e. an intervention restores the bridge component's condition back to an 'as good as new' condition. However with the demonstrated method, it is expected for more accurate results with the increasing number and better recorded data. 


\section{Chapter 4 - Markov Bridge Model}

\subsection{Introduction}

The modelling of the bridge degradation using the Markov modelling technique has been widely adopted over the last 20 years. There are many Markov bridge models in the literature as discussed in chapter 2. However, most of these models are simple. In this chapter, a Markov bridge model is developed that uses the degradation rates determined from the previous chapter to model the future asset condition. The model is considered in much more detail than other bridge models available in the literature by accounting for the inspection, servicing interval, repair delay time and opportunistic maintenance. The model can also be used to investigate the effects of different maintenance strategies. The whole life cycle costs can also be estimated using the model.

\subsection{Development of the continuous-time Markov bridge model}

\subsubsection{Elemental model}

\subsubsection{Degradation process}

As discussed in the previous chapter, there are four states a bridge component can be considered to reside in. Figure 4.1 shows a four-state Markov diagram that models the degradation process of a single bridge element. In order to satisfy the Markovian property, the distribution of time transitioning between 
states is assumed to be the exponential distribution. This means that the deterioration rates i.e. transition rates between states are constant and are represented by $\lambda_{1}, \lambda_{2}, \lambda_{3}$.

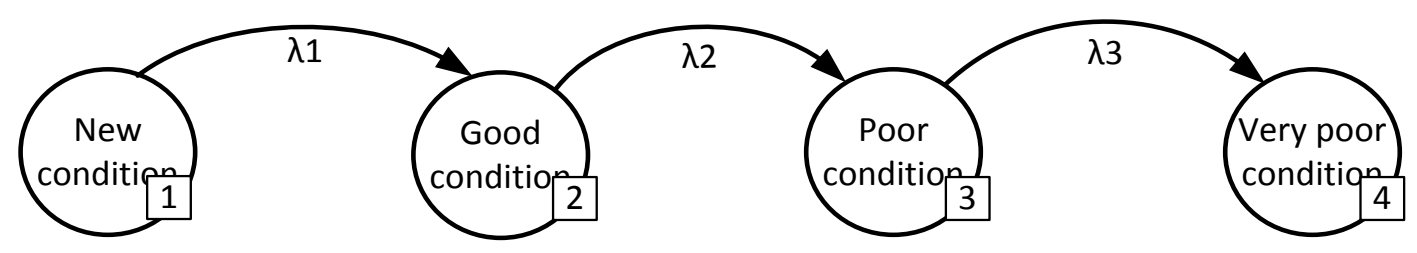

Figure 4.1: Markov state diagram - degradation process

\subsubsection{Inspection process}

All bridges and their components are normally inspected after a certain period of time. At the point of inspection, the current state of the bridge component is identified. If a change in the state of the element (i.e. the moving of the state from poor to very poor) happens in between two inspections, the failure is unrevealed until the second inspection. Four more states (State 5 to State 8) are added to represent the states where the actual condition of an element is revealed following an inspection, these states are shown in Figure 4.2.

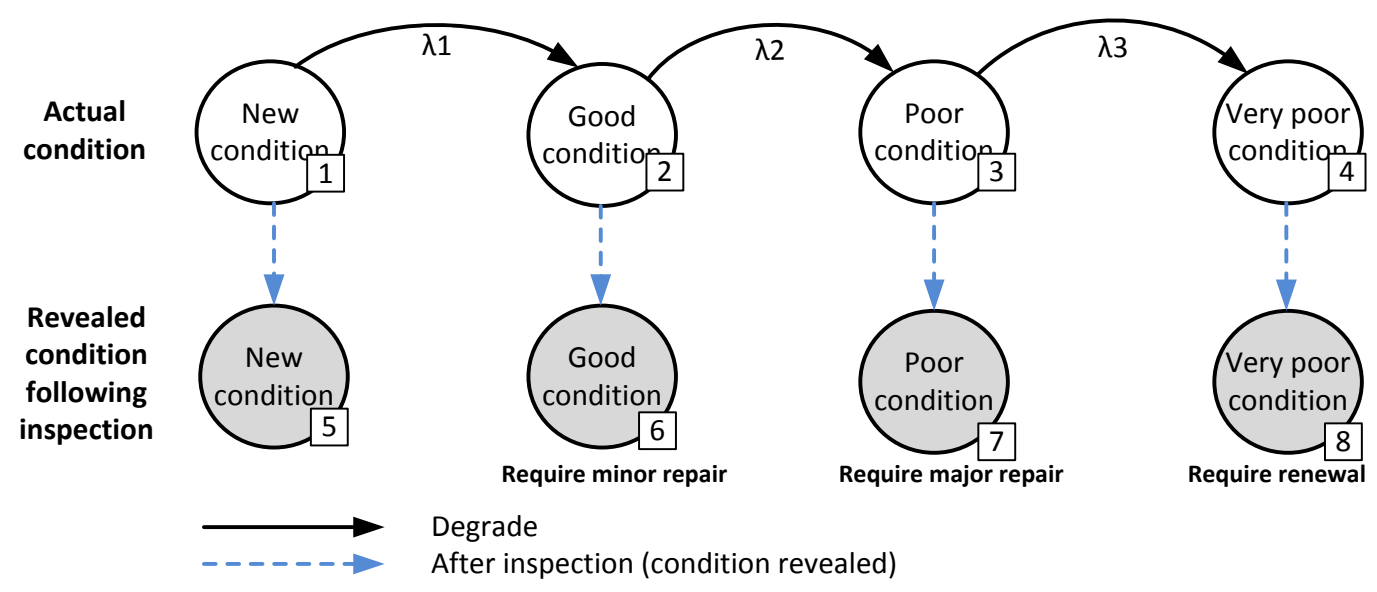

Figure 4.2: Markov state diagram - inspection process

\subsubsection{Repair process}

After an inspection, a maintenance decision can be made to repair the component or it can be left to deteriorate to a poorer state. For example, following an inspection, if it is revealed that the component is in State 2, the element can either be scheduled for repair (State 6 ) or left to deteriorate to a poorer state. The option to carry out repair is achieved by enabling the repair process represented by the arrow with a repair rate $v_{1}$ connecting State 6 
and State 1 in Figure 4.3. In contrast, if the fore-mentioned arrow is removed, the repair process is disabled. This means that even if the component is discovered to be in the state where minor repair is possible, no action is being taken and the component continues to deteriorate. A similar process applies when the component deteriorates to a state where a major repair is necessary to return it to the as new condition (State 7). The options for repair or no repair is again set by the repair process represented by an arrow connecting States 7 and 1 . Note that State 8 is when the component is revealed to be in a very poor condition and further deterioration is not acceptable. The component should be repaired as soon as it reaches this level of condition and the repair process between State 8 and State 1 should always be enabled.

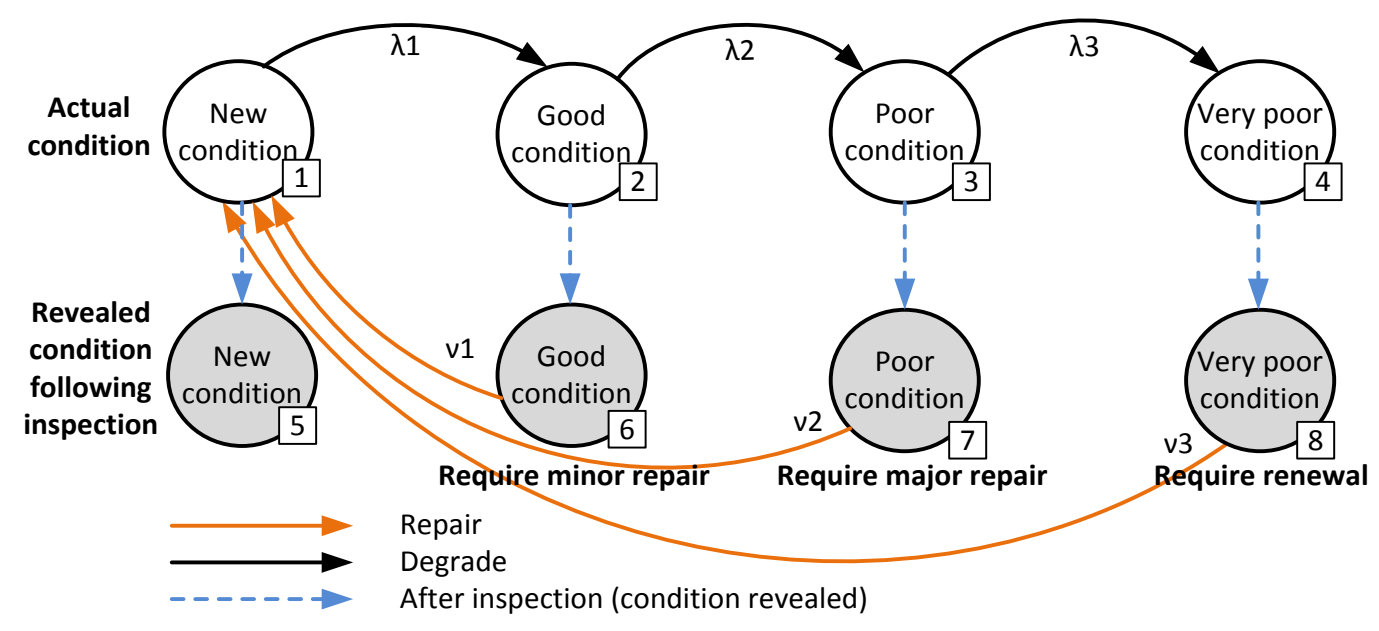

Figure 4.3: Markov state diagram - a single bridge element

\begin{tabular}{|c|l|l|}
\hline Strategy & \multicolumn{1}{|c|}{ Action } & \multicolumn{1}{|c|}{$\begin{array}{c}\text { Model } \\
\text { representation }\end{array}$} \\
\hline $\begin{array}{c}\text { Strategy } \\
1\end{array}$ & $\begin{array}{l}\text { Repair as soon as the component is } \\
\text { identified to be in a state where minor } \\
\text { repair is necessary, then it is carried out. }\end{array}$ & $\begin{array}{l}\text { Repair arrows } v_{1}, v_{2} \\
v_{3} \text { are kept as shown } \\
\text { in the figure }\end{array}$ \\
\hline $\begin{array}{c}\text { Strategy } \\
2\end{array}$ & $\begin{array}{l}\text { Repair when the component is identified to } \\
\text { be in the state where major repair is } \\
\text { required i.e. repair when the component } \\
\text { reaches the poor condition. }\end{array}$ & $\begin{array}{l}\text { Repair arrow } v_{1} \text { is } \\
\text { removed }\end{array}$ \\
\hline $\begin{array}{c}\text { Strategy } \\
3\end{array}$ & $\begin{array}{l}\text { Repair when the component is identified as } \\
\text { being in a state where renewal is needed } \\
\text { i.e. repair when the component reaches the } \\
\text { very poor condition. }\end{array}$ & $\begin{array}{l}\text { Repair arrows } \mathrm{v}_{1} \text { and } \\
\mathrm{v}_{2} \text { are removed }\end{array}$ \\
\hline $\begin{array}{c}\text { Strategy } \\
4\end{array}$ & $\begin{array}{l}\text { No repair, component is allowed to } \\
\text { deteriorate without any interventions }\end{array}$ & $\begin{array}{l}\text { Repair arrows } \mathrm{v}_{1}, \mathrm{v}_{2} \\
\text { and } \mathrm{v}_{3} \text { are removed }\end{array}$ \\
\hline
\end{tabular}

Table 4.1: Maintenance strategies possible in the elemental bridge model 
There are four maintenance strategies possible in this model and these are described in Table 4.1. The model shown in Figure 4.3 is a complete elemental Markov model that, effectively, models two phases in the component's life: the first phase is the continuous phase, modelling the degradation and repair processes between any two inspections, and the second is a discrete phase at the point where the condition of a bridge element is revealed by inspection and the decision of whether to repair or not is made.

\subsubsection{Bridge model}

Based on the same concept as the elemental model, the bridge model can then be built. It is worth noting that the number of states in a Markov model increases exponentially as the number of components in the model increases. In particular, there are 4 condition states for one bridge element, if there are $n$ bridge elements being modelled then the system of all components requires $4^{n}$ states. Each state is a unique system state representing a unique combination of component states as demonstrated below, for example, State 1 is when all the components are in the 'as new' condition, State 2 is when one of the components is in a good condition and all others are in the as new condition, etc.

$\begin{array}{ccccc}\text { State } & \text { Component 1 } & \text { Component 2 } & \cdots & \text { Component n } \\ 1 & \text { As new } & \text { As new } & \cdots & \text { As new } \\ 2 & \text { Good } & \text { As new } & \ldots & \text { As new } \\ \vdots & \vdots & \vdots & \vdots & \vdots \\ 4^{n} & \text { Poor } & \text { Poor } & \cdots & \text { Poor }\end{array}$

It is not possible to illustrate the complete bridge model graphically due to its size and complexity. Therefore, a system of two components (two main girders system) is used to illustrate the Markov states, this is shown in Figure 4.4. Each bridge main girder can be in four possible conditions, thus there are $4^{2}=16$ possible Markov states. At the point of inspection, the conditions of the components are revealed, therefore an extra 16 states are added to the model representing the states where the component conditions are actually known. In Figure 4.4, the degradation and repair transitions between the states are represented by solid arrows, and inspection transitions are denoted by dashed arrows. The shaded states are the states for which the bridge element conditions are revealed from inspection. For example, in state 24, 
it is revealed that after the inspection, the main girder $1(\mathrm{G} 1)$ is in a good (G) condition while the main girder $2(G 2)$ is in a very poor (VP) condition.

If the maintenance strategy is to repair the components as soon as they reach a state where repair is possible, then the repair process will restore the girders' conditions to 'as good as new'. This is represented by the repair process from state 24 to state 1. It is worth noting that for different maintenance strategies, the Markov model states are the same however the repair transitions are different. Figure 4.5 and Figure 4.6 illustrate the model for the same two girder system that is managed under maintenance strategies 2 and 3 respectively. Note that the degradation transitions are the same as illustrated in Figure 4.4 and are not therefore shown in those figures for the sake of clarity.

A computer program was written in Matlab to aid the process of generating the larger and more detailed Markov bridge model to include more components. The software first generates all the possible model states then generates all the transitions possible governed by a specified maintenance strategy. The software allows the model of any size to be generated, thus the size of the model is only restricted by the memory size available on a particular machine. However, the solution time increases significantly as the size of the model increases. 


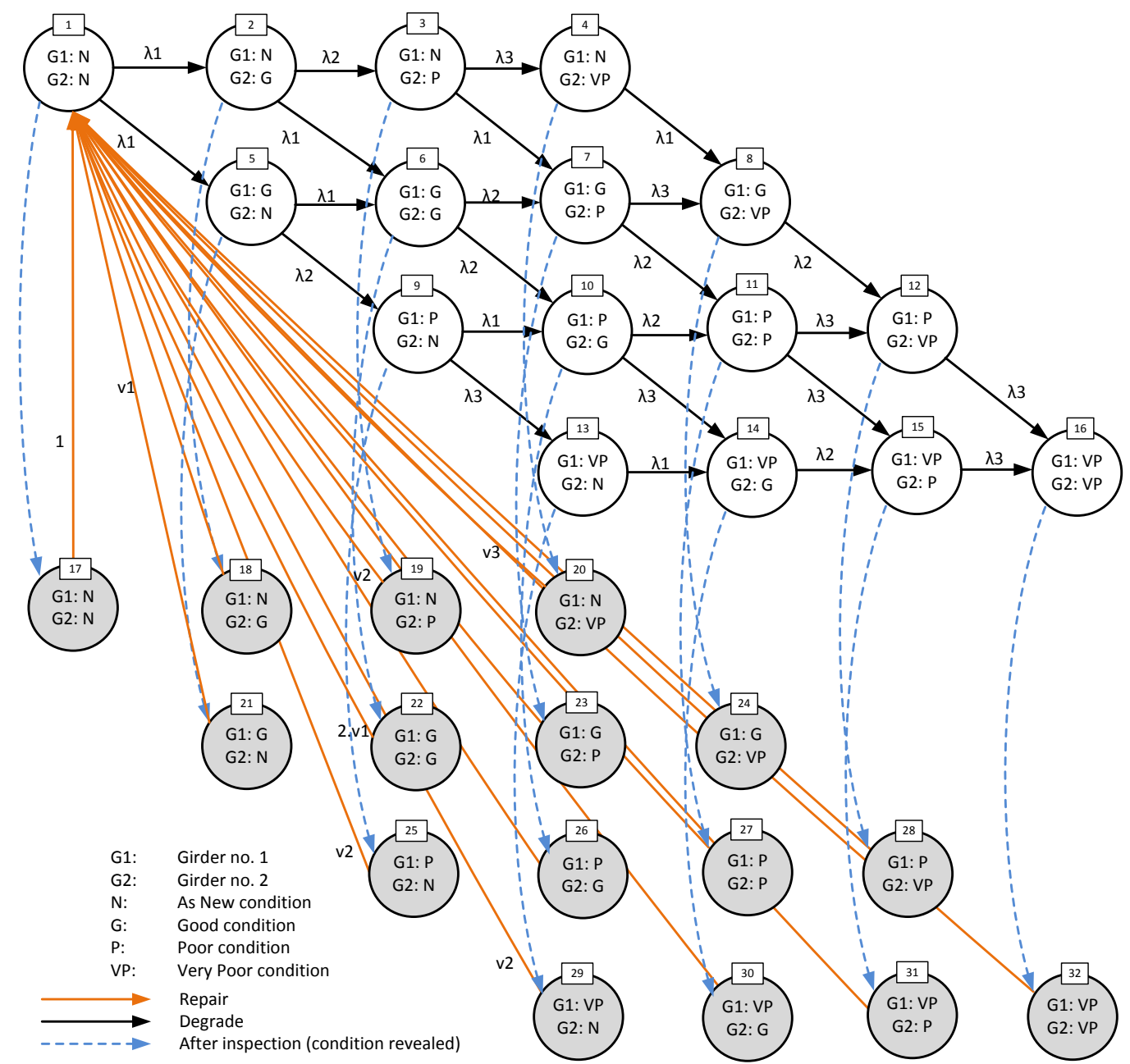

Figure 4.4: Markov bridge model for two main girders assuming strategy 1: repair as soon as any repairs are necessary 


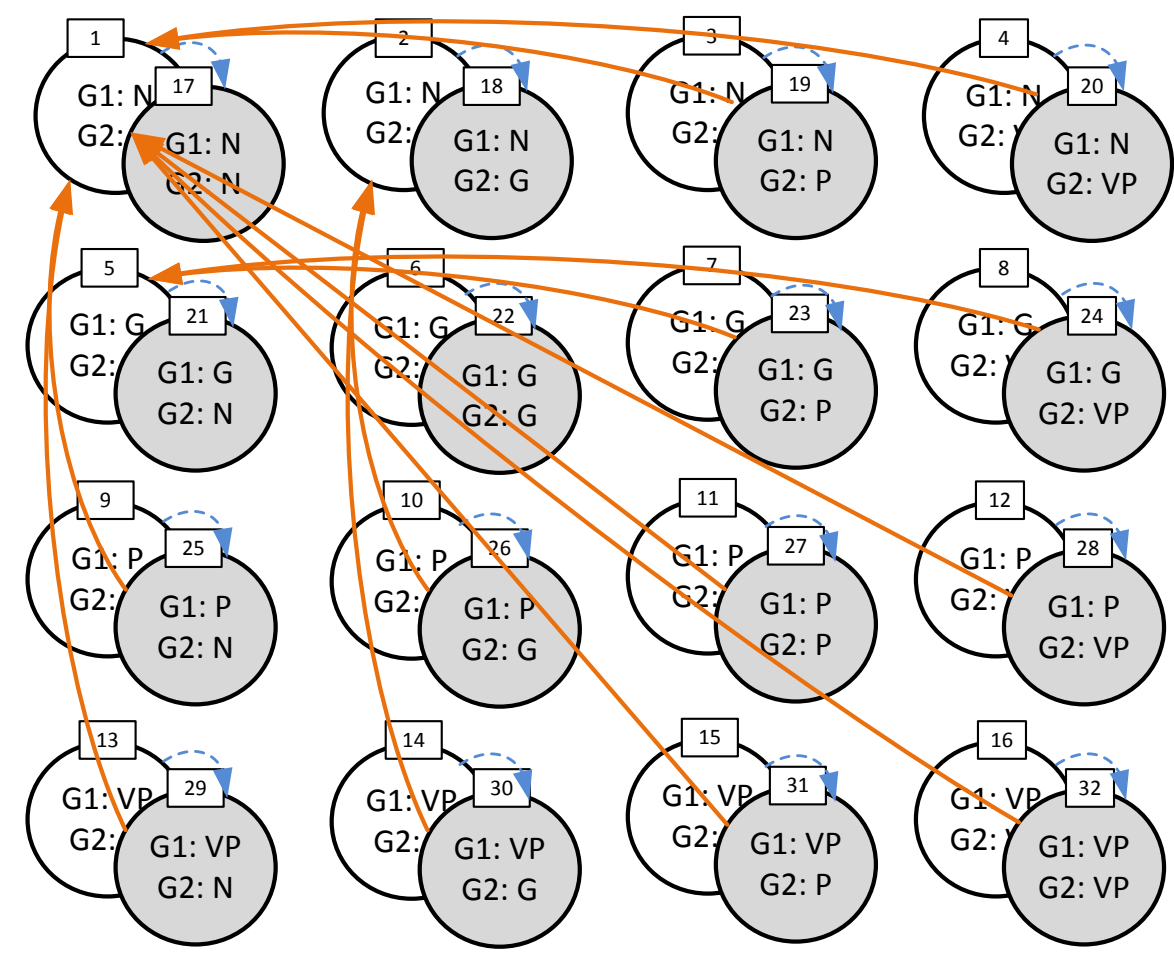

Figure 4.5: Markov bridge model for two main girders assuming strategy 2: repair when the component reaches the condition where major repair is needed

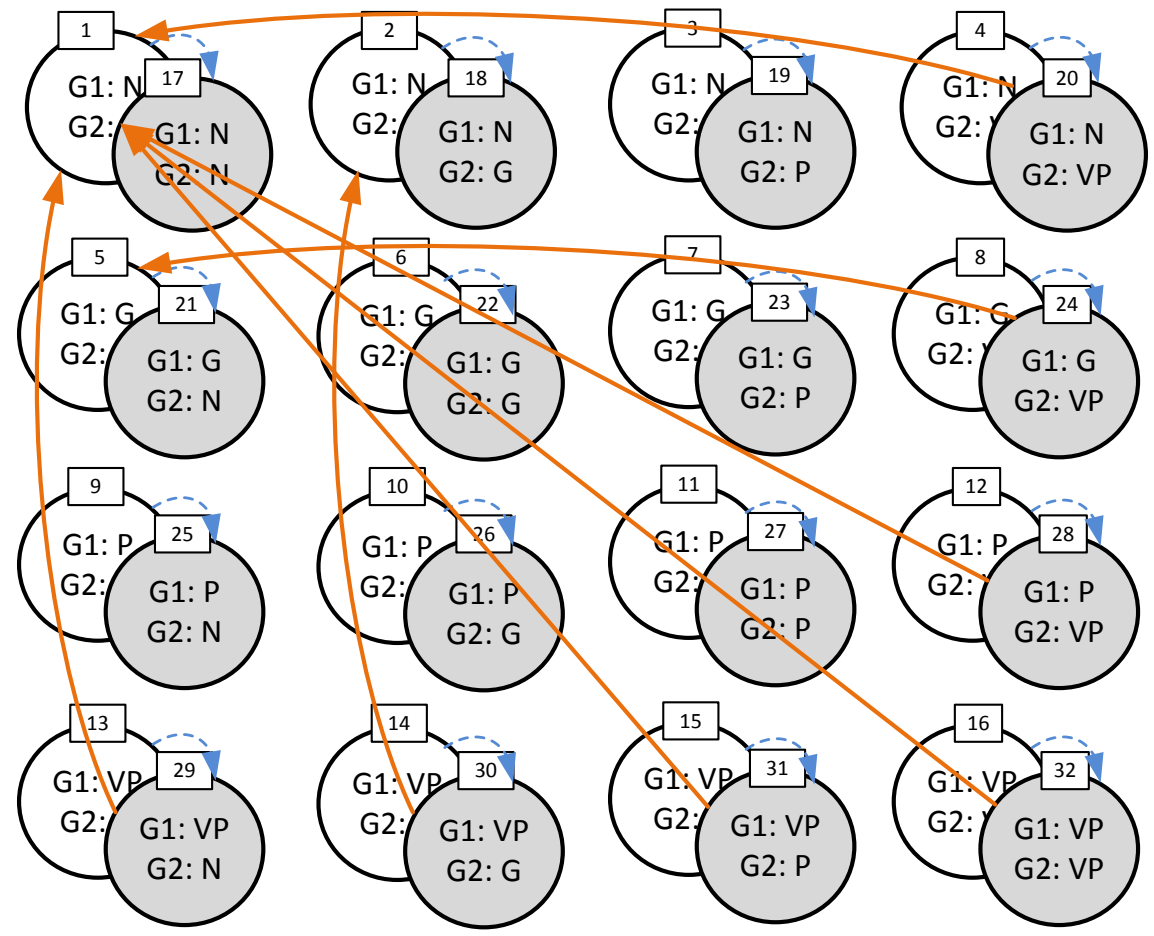

Figure 4.6: Markov bridge model for two main girders assuming strategy 3: repair when the component reaches the condition where renewal is needed 


\subsubsection{Opportunistic maintenance}

Generally, there are a couple of advantages (Samhouri, 2009) for applying opportunistic maintenance: 1 ) to extend equipment lifetime or at least the mean time to the next failure whose repair may be costly. It is expected that this maintenance policy can reduce the frequency of service interruption and the many undesirable consequences of such interruption, and 2) to take advantage of the resources, efforts and time already dedicated to the maintenance of other parts in the system in order to cut cost.

Since the bridge model consists of many different elements, the conditions and deterioration rates of these elements are different hence the times when interventions are required are also different. If a component is being repaired, opportunistic maintenance considers carrying out repair on other components which have a deteriorated condition but would not normally instigate maintenance. This takes advantage of any possession time or preparation required by the major maintenance task to minimise longer term service disruption. Figure 4.7 shows again the model for two main girders with maintenance strategy 2 and identifies the states where opportunistic repair are possible. In particular, state 23 in the model represents the case that after an inspection the main girder 1 is discovered to be in a good condition and the main girder 2 is in a poor condition. Under maintenance strategy 2 , only the main girder 2 will be repaired, the repair process will bring the system to state 5 where the main girder 2 is now in the as new condition whilst the main girder 1 remains in the same condition. It is possible in this case to carry out opportunistic maintenance on main girder 1 , this will bring the system back to state 1 where both component conditions are restored to the 'as good as new' condition. The repair process represented by an arrow connecting State 23 and State 5 will be replaced by an arrow from State 23 to State 1 . Similarly, Figure 4.8 shows the Markov state diagram for opportunistic repair with maintenance strategy 3 . Again the process of generating the repair transitions for opportunistic maintenance is carried out automatically by the software. 


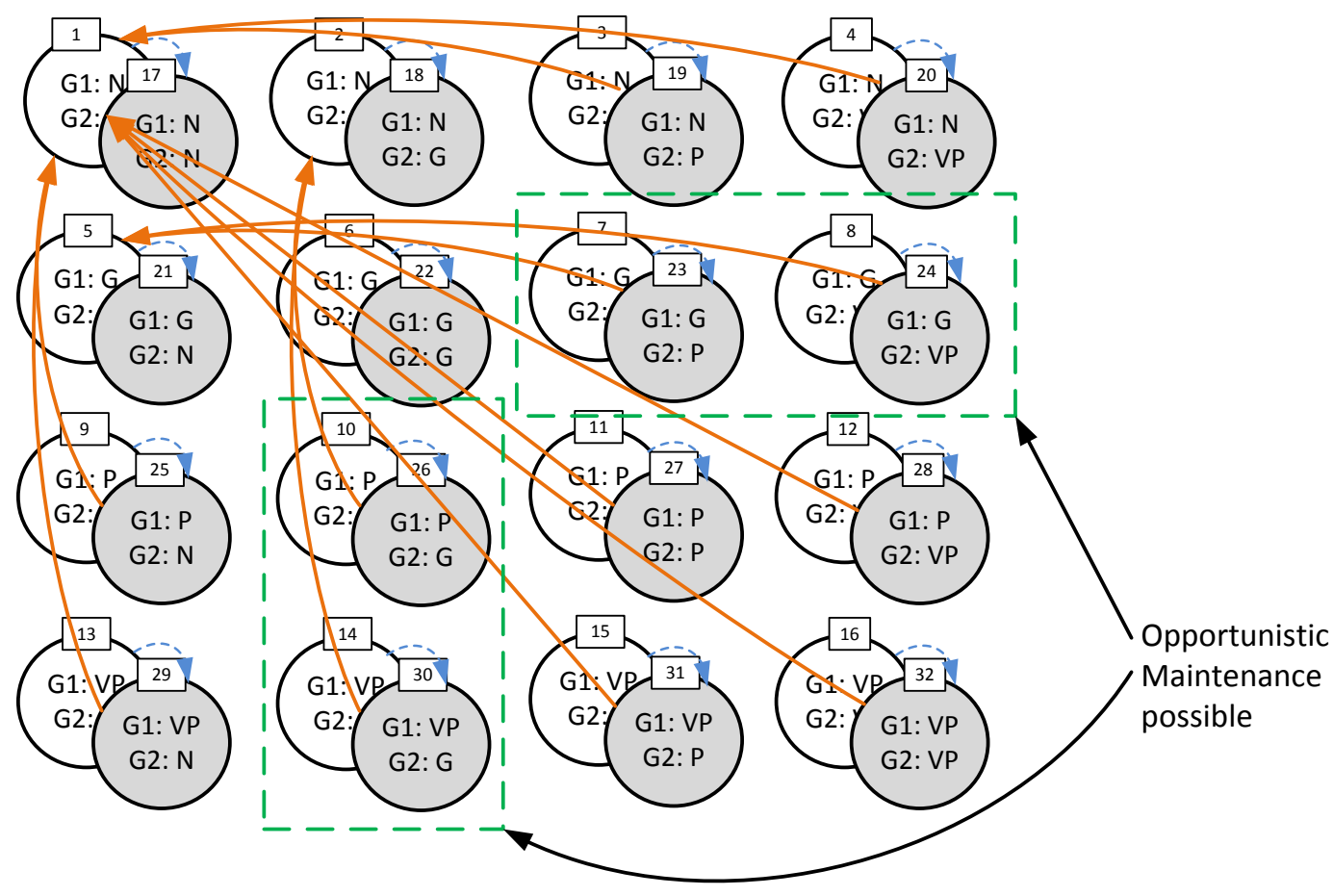

Figure 4.7: States where opportunistic maintenance are possible in a system consisting of two main girders operating under maintenance strategy 2

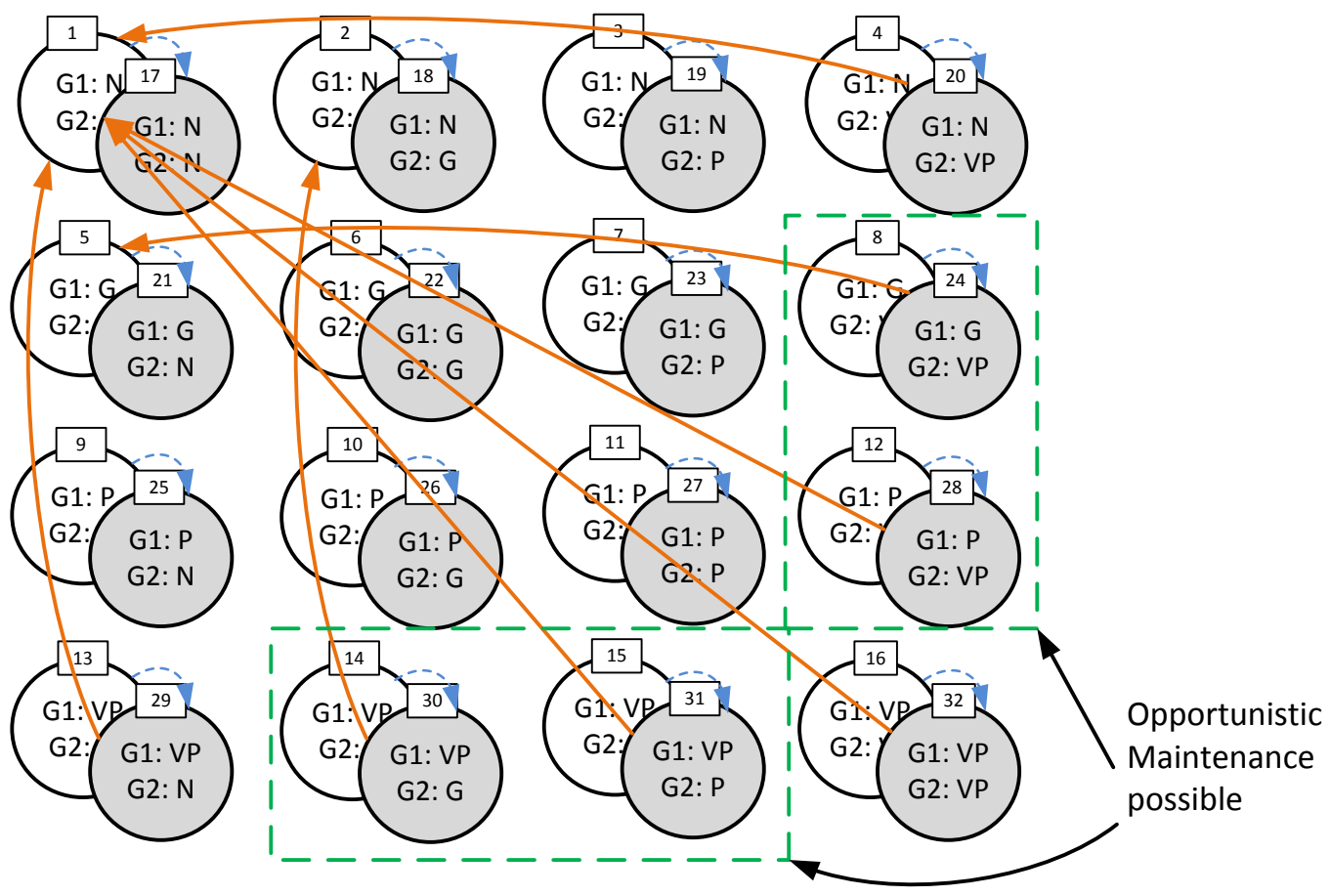

Figure 4.8: States where opportunistic maintenance are possible in a system consisting of two main girders operating under maintenance strategy 3 


\subsubsection{Environment factor}

Depending on the environment where the bridge components operate, the degradation rate will be different. There are three types of environment generally considered by the industry (Network Rail, 2010a), and their definitions are as followed:

- Aggressive: exposed to marine environments or harsh environments with repeat cycles of freezing and thawing, wetting and drying.

- Moderate: all bridges which do not fall into either of the two other categories.

- Benign: situated in a windy, non-marine, non-road environment which dries bridge without salt deposition.

Each type of structure in those environments would have different deterioration rates, therefore the deterioration rates should be adjusted accordingly with an adjustment factor. There was no study in the literature that quantifies the effects however, the experts opinion (Network Rail, 2010a) is that, for all types of structure, the component deteriorates three times faster in an aggressive environment compared with the benign environment; and the degradation rate is $30 \%$ less in a moderate environment compared with an aggressive environment. The estimated effects of environment on the deterioration rates of different materials are presented in a table given in Appendix B. Once the environment is specified in the model, the deterioration rate is adjusted by multiplying by the appropriate adjustment factor, this allows the model to effectively consider the effect of different deterioration rates.

\subsubsection{Servicing frequency}

Another factor that changes the degradation rate is the servicing frequency. Servicing work does not change the state of the component but helps slow down the deterioration rate and increases the time until the next intervention is needed. Servicing work includes: painting, pigeon proofing, cleaning debris. A simple study was carried out in the previous chapter, to determine the effects of servicing frequency on the deterioration rates by comparing the degradation rates of the same group of components that have a different servicing frequency recorded. A linear relationship was established to model this effect. Therefore, at a given servicing interval, the deterioration rate of a particular 
element is adjusted accordingly. The adjustment factors were discussed and presented in Chapter 3 - 3.6.2. The model is then capable of capturing and modelling the effects that a different servicing interval will have on the degradation process of bridge elements.

\subsubsection{Transition rates}

\subsubsection{Degradation rates}

The deterioration rates are attained by examining the database and maintenance records and analysing the times that each element takes to deteriorate to the point where a certain type of maintenance is required. These rates are obtained in Chapter 3 3.6.1, however they govern the process from the 'as new' state. The Markov model needs the transition rates between two adjacent states (good to poor, poor to very poor). The rate from state $i$ to state $j$ can be calculated as the inverse of the mean time reaching state $j$ from state $i, M T T F_{i, j}$ this can be estimated from:

$$
M T T F_{i, j}=M T T F_{1, j}-M T T F_{1, i}
$$

Giving:

$$
\lambda_{i j}=\frac{\lambda_{i} \lambda_{j}}{\lambda_{i}-\lambda_{j}}
$$

$\lambda_{i j}$ is the deterioration rate from state $i$ to state $j$ and is calculated as the inverse of $M T T F_{i, j}$. $\lambda_{i}$ is the deterioration rate from the new state to state $i$. $M T T F_{1, i}$ and $M T T F_{1, j}$ are taken as the mean values of the distributions obtained in Chapter 3 - 3.6.1. It is important to know that those distributions are Weibull distributions however the Markov model only allows constant transition rates i.e. exponential distribution to be modelled, therefore the mean values of the Weibull distributions obtained were used to estimate the transition rates of Markov bridge model. This is assumed good estimations since the $\beta$ values obtained for those Weibull distributions are very close to 1 (Chapter 3 - 3.6.1).

As mentioned previously, the degradation rate is affected by the environment and servicing frequency, thus the final step of deducing the degradation rate is by adjustment according to Equation (4.3). 


$$
\lambda_{i j}=\lambda_{i j} \times \mathrm{A}_{E} \times \mathrm{A}_{S}
$$

where

$\mathrm{A}_{E}$ is the adjustment factor according to different environment as given in Appendix $B$

$\mathrm{A}_{s}$ is the adjustment factor according to different servicing frequency as given in Chapter 3 -3.6.2

\subsubsection{Repair rates}

The repair rates, $v_{1}, v_{2}, v_{3}$ are included in the model as illustrated in Figure 4.3, representing the rates that bring the component from the good, poor, and very poor state back to the 'as new' state. The time to repair consists of two main components:

- the time to schedule the repair $\left(T_{S}\right)$

- the time of the actual repair work being carried out $\left(T_{R}\right)$

The time of repair is calculated as the duration of the repair carried out. The distribution of these repair times were obtained in Chapter 3 - 3.6.3. Again, the mean values of these distributions were used as the values of $T_{R}$.

The time to schedule the work is defined as the duration between when the work was identified as being necessary and when the work actually starts. $T_{S}$ is essentially a parameter in the model that dictates the delay of any repair works.

Thus the repair rate can be calculated as:

$$
v=\frac{1}{M T T R}=\frac{1}{T_{S}+T_{R}}
$$

\subsubsection{Transition rate matrix}

There are two phases in the model:

The first phase is the continuous phase between any two inspections, the system equation is governed by equation (4.5) where $Q$ is the matrix representing the probabilities of being in each state; $A$ is the transition rate matrix based on the deterioration rates and repair rates as given in Equation (4.5); and $\dot{Q}$ is the rate of change of probabilities at each state in the model. Note that the transition rate matrix given in Equation (4.6) is for a single bridge element. The transition rate matrix for a 
system containing two elements as illustrated in Figure 4.4 is given in Appendix C-1.

$$
[A]=\left[\begin{array}{cccccccc}
-\lambda_{1} & \lambda_{1} & 0 & 0 & 0 & 0 & 0 & 0 \\
0 & -\lambda_{2} & \lambda_{2} & 0 & 0 & 0 & 0 & 0 \\
0 & 0 & -\lambda_{3} & \lambda_{3} & 0 & 0 & 0 & 0 \\
0 & 0 & 0 & 0 & 0 & 0 & 0 & 0 \\
0 & 0 & 0 & 0 & 0 & 0 & 0 & 0 \\
v_{1} & 0 & 0 & 0 & 0 & -v_{1} & 0 & 0 \\
v_{2} & 0 & 0 & 0 & 0 & 0 & -v_{2} & 0 \\
v_{3} & 0 & 0 & 0 & 0 & 0 & 0 & -v_{3}
\end{array}\right]
$$

The second phase, corresponding to the point of inspection, is a discrete phase. At this point probabilities in the model are transferred between unrevealed condition states and known condition states according to Equation (4.7). $Q_{k}(t)$ and $Q_{k}^{\prime}(t)$ are the state probabilities immediately prior to following inspection respectively and $k$ represents the states where the component state is scheduled for a certain type of repair and $i$ represents the state of the corresponding unrevealed condition.

$$
\begin{aligned}
Q_{\mathrm{k}}^{\prime}(t) & =Q_{\mathrm{k}}(t)+Q_{\mathrm{i}}(t) \\
Q_{\mathrm{i}}^{\prime}(t) & =0
\end{aligned}
$$

\subsubsection{Expected maintenance costs}

Average repair costs for each type of maintenance work on each of the bridge elements of different materials were estimated from the database of previous work carried out (Chapter 3 - 3.6.4). The average cost of the maintenance is combined with the cost of any requirement for possession (note that this cost is different depending on asset route criticality). The total repair cost over the structure life period is then calculated by taking the product of the number of bridge element repairs of each severity and the average costs of such repairs. The number of bridge element repairs can be calculated by integrating the rate of transitions from each corresponding degraded state to the as new state over the specified life time, $\mathrm{T}$. The expected repair costs are given in Equation (4.8). The servicing and inspection cost are also considered, depending on the frequency of the inspections and services, these costs can easily be added to the total costs. In total, the total expected maintenance costs for a component is: 
Total expected maintenance cost $=$ Minor repair cost + Major repair cost + Replacement cost + Servicing cost + Inspection cost

$$
\begin{gathered}
=\int_{0}^{T} \mathrm{Q}_{k}^{i}(t) \cdot v_{1}^{i} d t \times C_{1}^{i}+\int_{0}^{T} \mathrm{Q}_{l}^{i}(t) \cdot v_{2}^{i} d t \times C_{2}^{i}+\int_{0}^{T} \mathrm{Q}_{m}^{i}(t) \cdot v_{3}^{i} d t \times \\
C_{3}^{i}+N_{S} C_{S}+N_{I} C_{I}
\end{gathered}
$$

where

$\mathrm{T}=$ Length of the prediction period (year)

$\mathrm{Q}_{k}^{i}(t)=$ Probability of the component $i$ requires minor repair at time $t$ and has been scheduled to repair (State $k$ )

$\mathrm{Q}_{l}^{i}(t)=$ Probability of the component $i$ requires major repair at time $t$ and has been scheduled to repair (State $l$ )

$\mathrm{Q}_{m}^{i}(t)=$ Probability of the component $i$ requires

replacement at time $t$ and has been scheduled to be

replaced (State $m$ )

$v_{1}^{i}=$ Minor repair rates of the component $i$

$v_{2}^{i}=$ Major repair rates of the component $i$

$v_{3}^{i}=$ Replacement rates of the component $i$

$C_{1}^{i}=$ Average Minor Repair Costs of the component $i$

$C_{2}^{i}=$ Average Major Repair Costs of the component $i$

$C_{3}^{i}=$ Average Replacement Costs of the component $i$

$\mathrm{C}_{\mathrm{s}}=$ Cost of servicing

$\mathrm{C}_{\mathrm{I}}=$ Cost of inspection

$\mathrm{N}_{\mathrm{S}}=$ Number of servicing over the whole prediction period

$N_{I}=$ Number of inspection over the whole prediction period

\subsubsection{Average condition of asset}

With four condition states used in the model, the average condition can be translated into a value by assigning the 'as new' state to have a value equals 1 ; the good, poor and very poor state have values 2,3 and 4 respectively. Since the model predicts the probability of bridge components being in different condition states, the average condition is calculated by multiplying the vector of these probabilities with a vector which contains scalar values from 1 to 4 . For example, if the probabilities of a bridge component being in an 'as new' and a good condition are 0.8 and 0.2 respectively, the average condition of the component is 1.2. This value is useful in the sensitivity analysis (investigating effects of different inspection and servicing intervals) and in the optimisation exercise, the minimisation of this value is the same as maximising the average asset condition ( 1 being the best and 4 being the worst condition). The average condition of an asset was assumed to be the average condition of all major elements modelled, and is calculate using Equation (4.9). 
where

$$
\sum_{i=1}^{N} \frac{\left[\begin{array}{llll}
\mathrm{Q}_{1}^{i}(T) & \mathrm{Q}_{2}^{i}(T) & \mathrm{Q}_{3}^{i}(T) & \mathrm{Q}_{4}^{i}(T)
\end{array}\right]}{N} \times\left[\begin{array}{l}
1 \\
2 \\
3 \\
4
\end{array}\right]
$$

$N=$ Number of bridge components in a bridge

$T=$ Length of the prediction period

$\mathrm{Q}_{j}^{i}(T)=$ Probability of the component $i$ in state $j$ at time $\mathrm{T}$

\subsubsection{Model assumptions}

Most of the model assumptions have been discussed throughout the development of the model and a summary of a list of the assumptions is given as follow:

- A repair will restore the element condition to the 'as new' condition,

- The model assumes a constant deterioration rate (transition rate),

- Constant inspection interval and servicing interval,

- Opportunistic maintenance is only carried out on the components of the same type (structure, material).

Other assumptions that are associated with a specific asset are:

- Scheduling time is assumed to be 12 months for a minor repair, 24 months for a major repair and 36 months for a renewal work.

- The servicing interval and deterioration rate are assumed to have a linear relationship.

\subsubsection{Model solutions}

A computer program was written in Matlab to construct and analyse the Markov bridge model. Simulations can be run on the complete bridge model to investigate the effects of different maintenance strategies. The lifetime duration, over which the predictions were made, was 60 years. A lifetime of 60 years is considered long enough to ensure that the maintenance strategy adopted takes actions which are in the longer-term interests of preserving the asset state. However, since in the modern era the frequency, weight and length of traffic as well as the maintenance policies applied to a bridge are commonly being reviewed and changed, it was not thought necessary to model a longer period. 
The transitions in the model are represented by rates. The inspection transitions were modelled as instantaneous shifts in probability at discrete times. These rates form a system of differential equations that was solved by a $4^{\text {th }}$ order Runge-Kutta method with variable time step to speed up the process. The step size decides the accuracy of the model solutions. The step size must be small enough that a two-state-jump does not occur within the given time step. Given the slow degradation rate of the bridge assets, it is believed that a 0.01 year ( 7 days) time step is reasonably small enough. Therefore the initial time step is assumed to be 0.01 year and the average step size over the whole solution routine was 0.03 years. The integration procedure gives the probability of the bridge component being in the different states and the probability of being in a particular condition can be found by adding the probabilities of the being all in all the states that represent that condition. A 2,048 Markov state model was generated in this study and the computation time was about 1 minute. 


\subsection{Model Application}

\subsubsection{Asset selection}

This section presents the results obtained for a selected typical metal underbridge structure and demonstrates the capabilities of the Markov bridge model developed. The bridge's main components include: concrete deck, metal bearing, metal main girders, masonry abutments. Their initial conditions are illustrated in Figure 4.9. Bridge components such as external main girders (MGE), bearings (BGL) and abutments (ABT) can be grouped together to reduce the number of model states. A Markov bridge model of $2 \times 4^{5}=2048$ states was generated for the analysis.

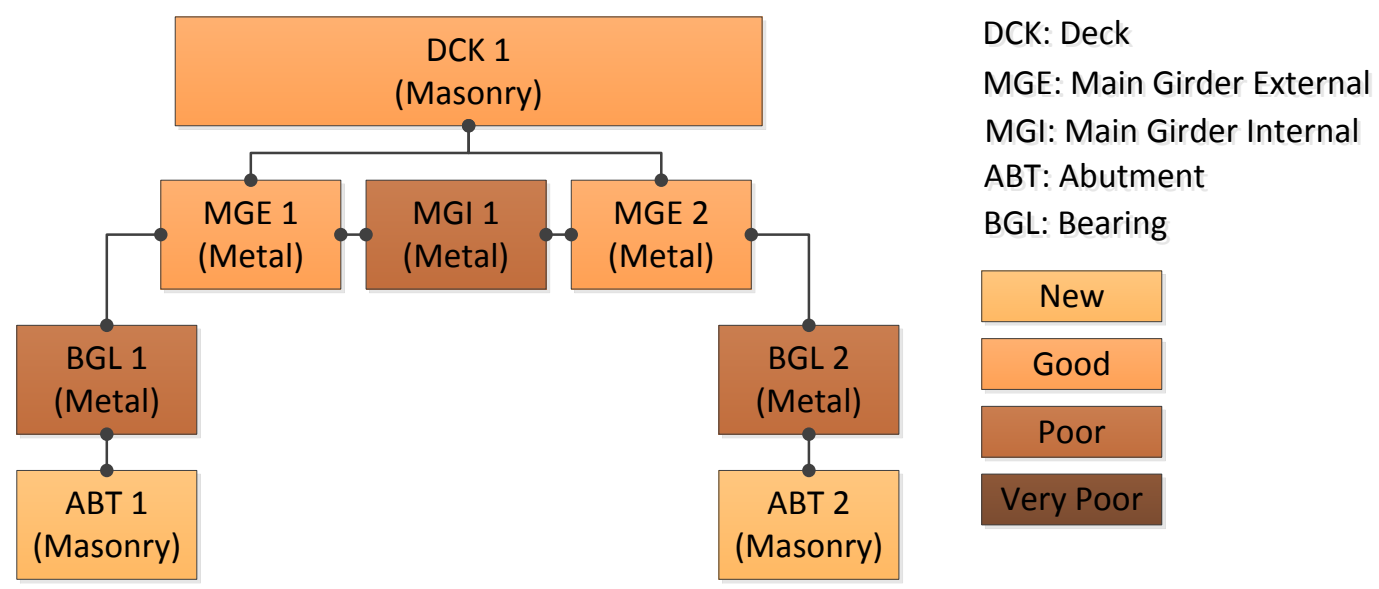

Figure 4.9: Structural arrangement of a typical metal underbridge

\subsubsection{Model parameters}

\begin{tabular}{|l|l|}
\hline \multicolumn{1}{|c|}{ Model parameters } & \multicolumn{1}{c|}{ Values } \\
\hline \multirow{4}{*}{ Maintenance strategy } & Strategy 1 \\
\cline { 2 - 2 } & Strategy 2 \\
\cline { 2 - 2 } & Strategy 2 (with opportunistic maintenance) \\
\cline { 2 - 2 } & Strategy 3 \\
\cline { 2 - 2 } & Strategy 3 (with opportunistic maintenance) \\
\hline Inspection interval & 6 years \\
\hline Servicing interval & 6 years \\
\hline Minor repair delay time & 1 year \\
\hline Major repair delay time & 2 years \\
\hline Renewal delay time & 3 years \\
\hline Operating environment & Aggressive \\
\hline
\end{tabular}

Table 4.2: Model parameters

Table 4.2 shows the model parameters. The analysis example presented in this section considers 5 repair strategies. Note that the model is capable of modelling the fourth strategy (as shown in Table 4.1) which is letting the asset to deteriorate without any interventions, however, this is considered impractical and this 
strategy is excluded from the analysis. Other model coreparameters such as the deterioration rates and the repair times are obtained from the results presented in the previous chapter.

\subsubsection{Effects of a specific maintenance strategy}

Strategy 1 (Repair as soon as the component is identified to be in a state where minor repair is necessary).

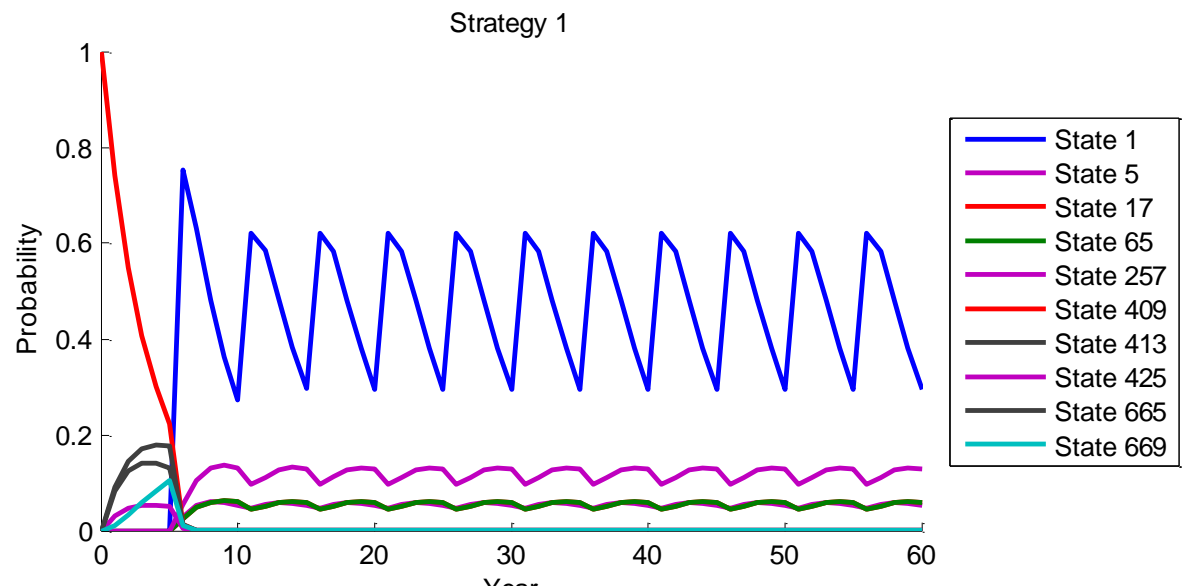

\begin{tabular}{|c|c|c|c|c|c|c|c|c|}
\hline State & DCK & MGE1 & MGI1 & MGE2 & BGL1 & BGL2 & ABT1 & ABT2 \\
\hline 1 & As new & As new & As new & As new & As new & As new & As new & As new \\
\hline 5 & As new & As new & As new & As new & Good & Good & As new & As new \\
\hline 17 & As new & Good & As new & Good & As new & As new & As new & As new \\
\hline 65 & As new & As new & Good & As new & As new & As new & As new & As new \\
\hline 257 & Good & As new & As new & As new & As new & As new & As new & As new \\
\hline 409 & Good & Good & Poor & Good & Poor & Poor & As new & As new \\
\hline 413 & Good & Good & Poor & Good & V.Poor & V.Poor & As new & As new \\
\hline 425 & Good & Poor & Poor & Poor & Poor & Poor & As new & As new \\
\hline 665 & Poor & Good & Poor & Good & Poor & Poor & As new & As new \\
\hline 669 & Poor & Good & Poor & Good & V.Poor & V.Poor & As new & As new \\
\hline
\end{tabular}

Figure 4.10: Probabilities of being in different model states for the bridge model under maintenance strategy 1.

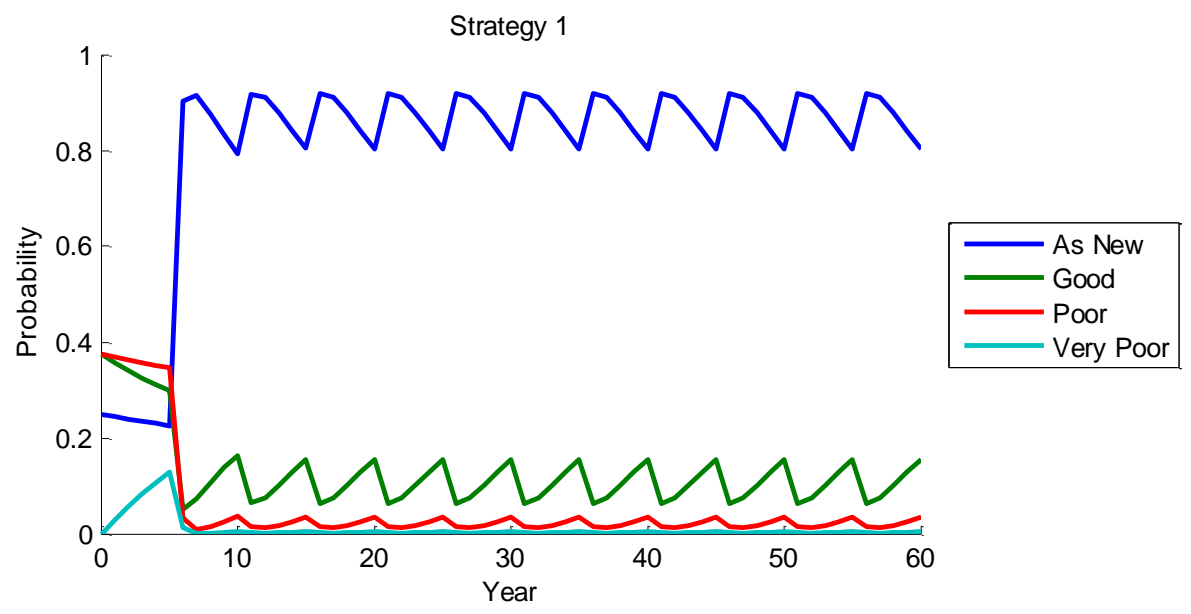

Figure 4.11: Average probabilities of being in different conditions states for the whole bridge under maintenance strategy 1 
Strategy 2 (Repair when the component is identified to be in the state where major repair is required i.e. repair when the component reaches the poor condition)

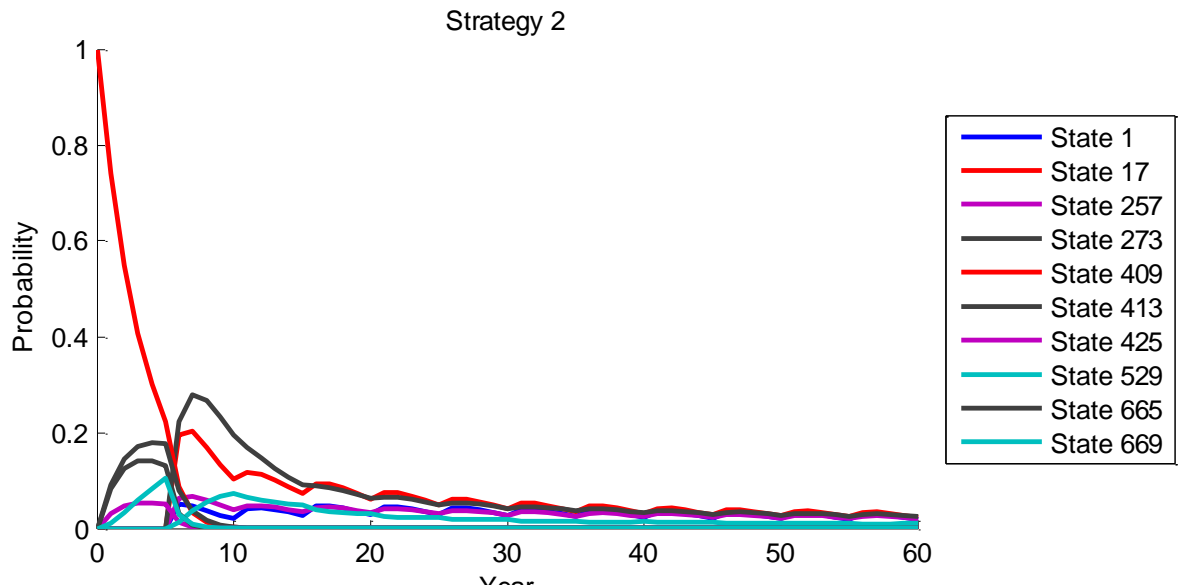

\begin{tabular}{|c|c|c|c|c|c|c|c|c|}
\hline State & DCK & MGE1 & MGI1 & MGE2 & BGL1 & BGL2 & ABT1 & ABT2 \\
\hline 1 & As new & As new & As new & As new & As new & As new & As new & As new \\
\hline 17 & As new & Good & As new & Good & As new & As new & As new & As new \\
\hline 257 & Good & As new & As new & As new & As new & As new & As new & As new \\
\hline 273 & Good & Good & As new & Good & As new & As new & As new & As new \\
\hline 409 & Good & Good & Poor & Good & Poor & Poor & As new & As new \\
\hline 413 & Good & Good & Poor & Good & V.Poor & V.Poor & As new & As new \\
\hline 425 & Good & Poor & Poor & Poor & Poor & Poor & As new & As new \\
\hline 529 & Poor & Good & As new & Good & As new & As new & As new & As new \\
\hline 665 & Poor & Good & Poor & Good & Poor & Poor & As new & As new \\
\hline 669 & Poor & Good & Poor & Good & V.Poor & V.Poor & As new & As new \\
\hline
\end{tabular}

Figure 4.12: Probabilities of being in different model states for the bridge model under maintenance strategy 2

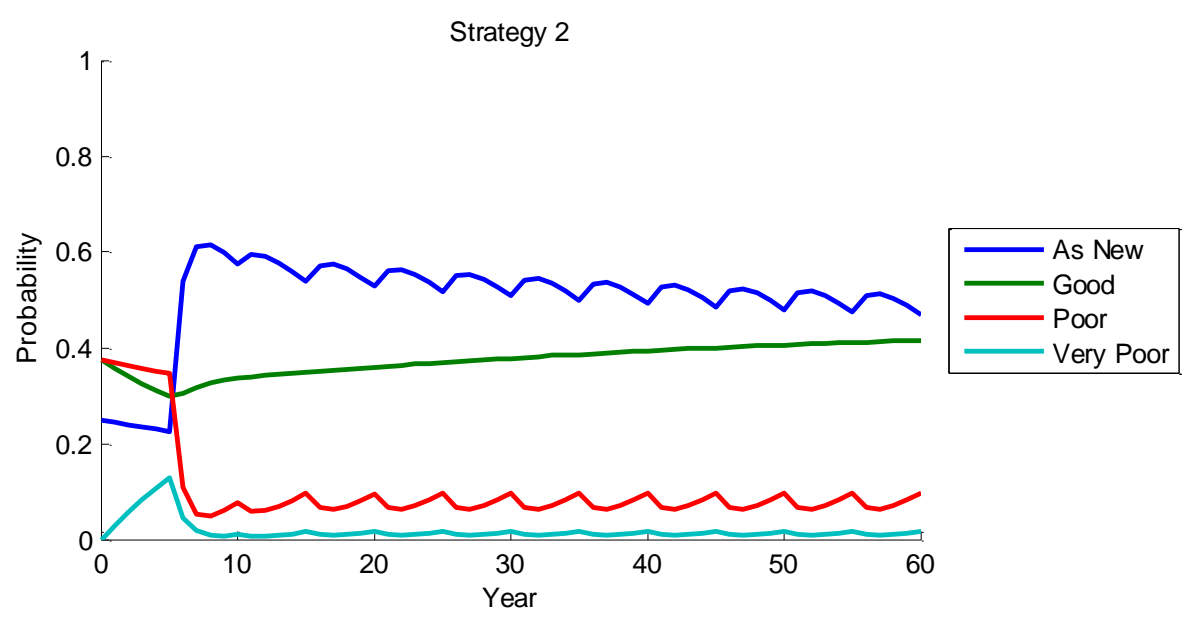

Figure 4.13: Average probabilities of being in different conditions states for the whole bridge under maintenance strategy 2 
Strategy 3 (Repair when the component is identified as being in a state where renewal is needed i.e. repair when the component reaches the very poor condition)

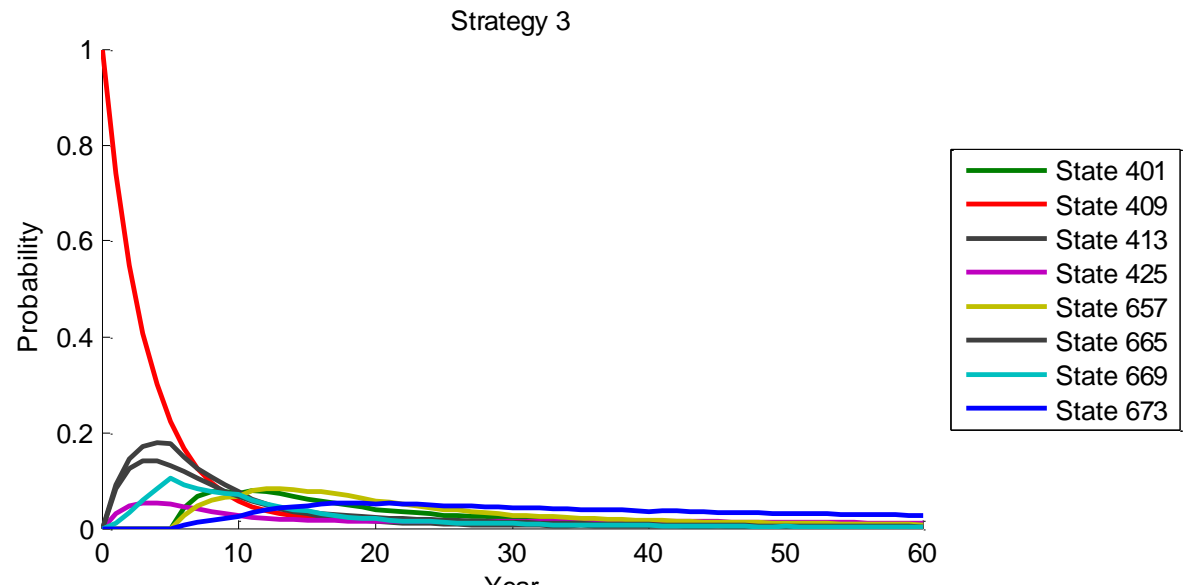

\begin{tabular}{|c|c|c|c|c|c|c|c|c|}
\hline State & DCK & MGE1 & MGI1 & MGE2 & BGL1 & BGL2 & ABT1 & ABT2 \\
\hline 401 & Good & Good & Poor & Good & As new & As new & As new & As new \\
\hline 409 & Good & Good & Poor & Good & Poor & Poor & As new & As new \\
\hline 413 & Good & Good & Poor & Good & V.Poor & V.Poor & As new & As new \\
\hline 425 & Good & Poor & Poor & Poor & Poor & Poor & As new & As new \\
\hline 657 & Poor & Good & Poor & Good & As new & As new & As new & As new \\
\hline 665 & Poor & Good & Poor & Good & Poor & Poor & As new & As new \\
\hline 669 & Poor & Good & Poor & Good & V.Poor & V.Poor & As new & As new \\
\hline 673 & Poor & Poor & Poor & Poor & As new & As new & As new & As new \\
\hline
\end{tabular}

Figure 4.14: Probabilities of being in different model states for the bridge model under maintenance strategy 3

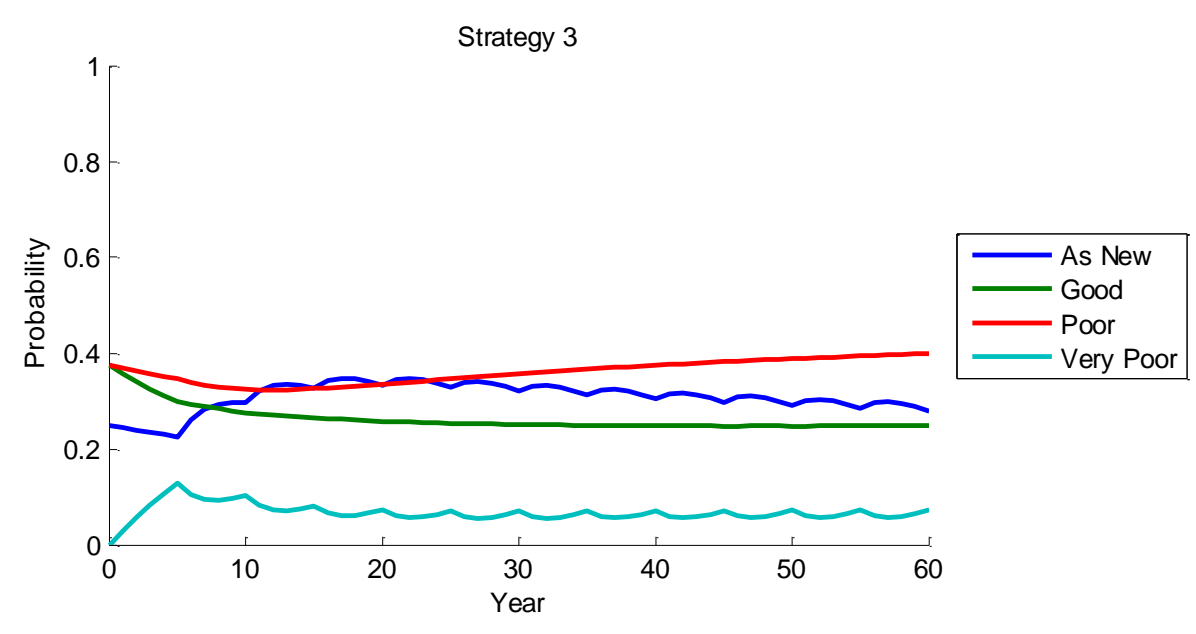

Figure 4.15: Average probabilities of being in different conditions states for the whole bridge under maintenance strategy 3

The result from the application of maintenance strategy 1 is shown in Figure 4.10, for strategies 2 and 3, the plots can be seen in Figure 4.12 and Figure 4.14 respectively. These plots show the probability of the bridge model being in different model states over the 60 year period. Each state in the model is a unique combination of all the bridge element conditions, the 
mapping of some of the model states and the element conditions can be seen in the tables below the graphs. It can be seen that, the model starts with the probability of 1 being in state 409, this is the initial condition of the bridge elements.

As the inspection period was set to every 6 years. The figure shows that during the first 6 years, the probability of the bridge model being in state 409 decreases and the probabilities of being in states 413, 425, 665 and 669 increases, these states represent the deterioration of the bearings, the girders and the deck. By the end of the first 6 years, the probability of all the components remaining in the same conditions as the initial conditions is only about $25 \%$. The probability that the bearings (BGL) deteriorate to the very poor state (state 413 ) is almost $20 \%$ whilst the likelihood of the deck or any main girder deteriorate to worse states is about $15 \%$ and $5 \%$ respectively. Note that the probability of being in any other state is less than $5 \%$ was not included in the plot.

Whilst Figure 4.10 shows the probability of the bridge model being in different models states under strategy 1, Figure 4.11 shows the probability of the entire bridge being in each of the four condition states. This probability is the average probability of all the bridge elements being in each four conditions, and is obtained by grouping the model states together. The repair process can be clearly seen after the 6th year when the probability of the components being in the as new condition increases and the probabilities of being any worse conditions decreases. It is expected with this maintenance strategy, that there is an average probability of $85 \%$ that the bridge asset will be operating in the 'as new' condition (state 1 ). This is because the strategy schedules repairs as soon as any degradation is revealed, resulting in a high probability of the component being in the 'as new' condition. Similarly, the probabilities of the bridge model being in each of the four condition states for strategies 2 and 3 are illustrated in Figure 4.13 and Figure 4.15.

The effects of maintenance can be seen in the 'wave' nature of the plots. The peak of the 'wave' is when the inspection happens and the condition of the component is revealed. Following this point, any revealed failures are scheduled for repair thus the probability of being in the 'as new' condition increases. A certain time after the repair, as the component continues to deteriorate, 
the probabilities of being in poorer conditions again increases. This process is what creates the 'wave' shape in the plot. After the next inspection when the component condition is revealed, the process is repeated again.

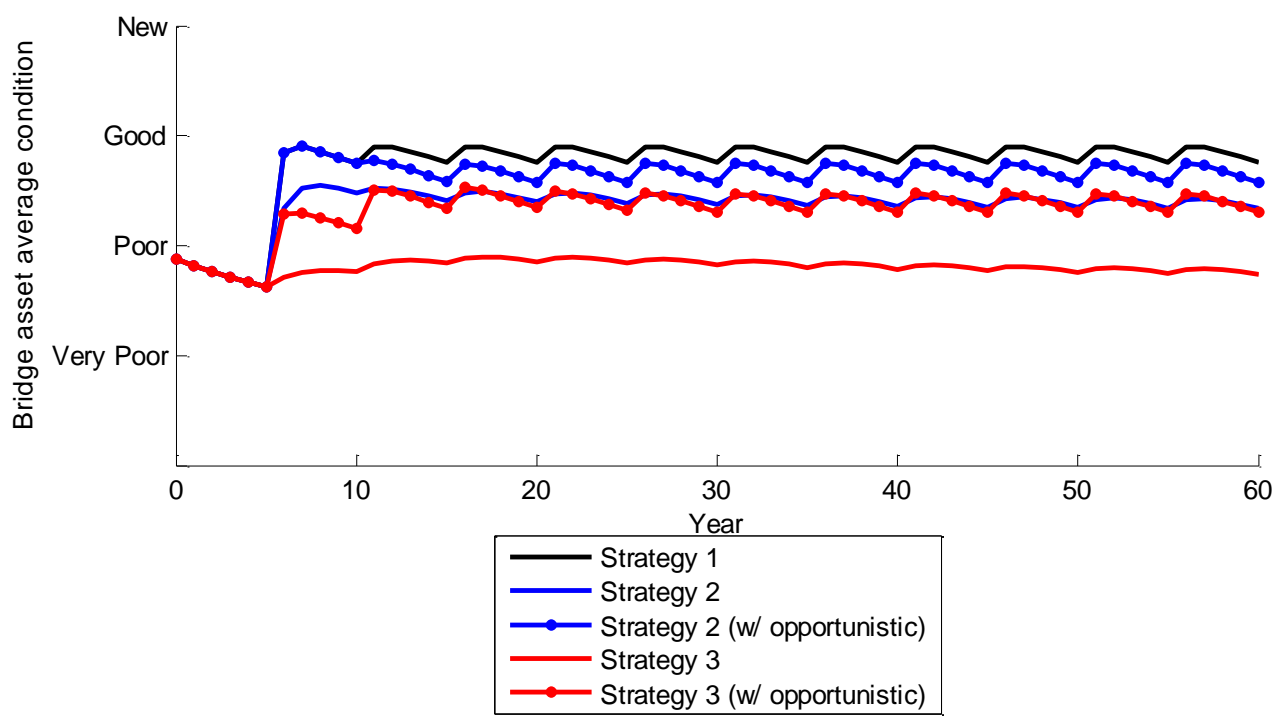

Figure 4.16: The effects of all intervention strategies

The effects of all 5 maintenance strategies on the average asset condition can be seen in Figure 4.16. For strategies 2 and 3, since the condition that triggers maintenance is lower, the probabilities of being in the as new state decrease and the probabilities of components being in poorer states are higher. This means that the average condition of assets would be lower for these two strategies. It can also be seen that opportunistic maintenance improves the average condition of the asset. The effects of opportunistic maintenance are explained in more details in the next section.

\subsubsection{Effects of opportunistic maintenance}

Figure 4.17 shows the effects of opportunistic maintenance on repair strategy 2 , which is to repair when the component reaches the poor condition. It can be seen in the top graph of Figure 4.17, after the first inspection, maintenance strategy carries out repair on the internal main girder (MGI) and the bearings (BGL) as these components are in the condition where major repair is necessary. This process brings the system to state 273 where the condition of MGI and BGL are now restored to 'as good as new'. Opportunistic maintenance also considers carrying out repairs on the deck (DCK) and external main girders (MGE) as these 
components have not yet reached the point where the repair is triggered for this maintenance strategy however they are in the state where repair is possible. This means that in this case all the components are being scheduled for repair at the same time, and all the components are expected to be in the 'as good as new' condition after the first repair, this is reflected in the bottom graph of Figure 4.17 as the probability of the model of being in state 1 increases to almost $80 \%$ after the first inspection.

As the result of applying opportunistic maintenance, it is more likely that the components will be operating in better conditions when compared with the case where opportunistic maintenance is not employed, the expected number of repairs will also increase resulting in higher maintenance costs at the start of the bridge modelling period.

Even though it is clear to see the components are more reliable when employing opportunistic maintenance, it is not clear how the whole life-cycle cost is affected. It is expected a high maintenance cost at the beginning of the lifetime as more repair needs to be done but since the component condition is improved early in the lifetime, the expected maintenance cost in the future is expected to reduce. This effect will be investigated in later section 4.3.6 when analysing the expected maintenance costs.

The effect of opportunistic maintenance, when applying maintenance strategy 3 , is demonstrated in Figure 4.18. Note that, for strategy 1 , opportunistic maintenance is unnecessary, this is because any component that reaches the good condition would be maintained immediately, thus, applying opportunistic maintenance for this strategy would produce the same results as illustrated in Figure 4.10. 

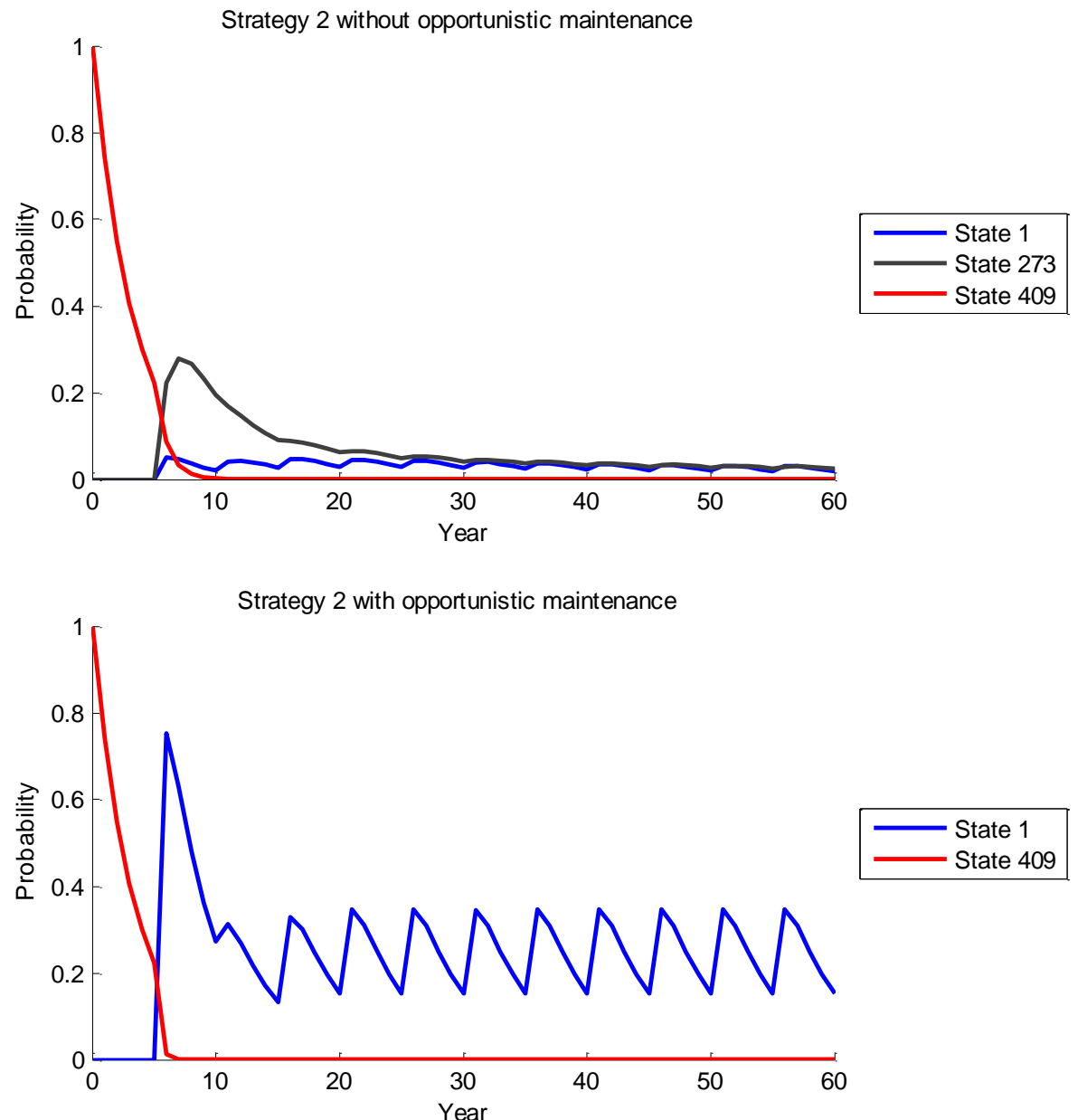

\begin{tabular}{|c|c|c|c|c|c|c|c|c|}
\hline States & DCK & MGE1 & MGI1 & MGE2 & BGL1 & BGL2 & ABT1 & ABT2 \\
\hline 1 & As New & As New & As New & As New & As New & As New & As New & As New \\
\hline 273 & Good & Good & As New & Good & As New & As New & As New & As New \\
\hline 409 & Good & Good & Poor & Good & Poor & Poor & As New & As New \\
\hline
\end{tabular}

Figure 4.17: Probabilities of being in different states of the bridge model under maintenance strategy 2 with and without opportunistic maintenance 

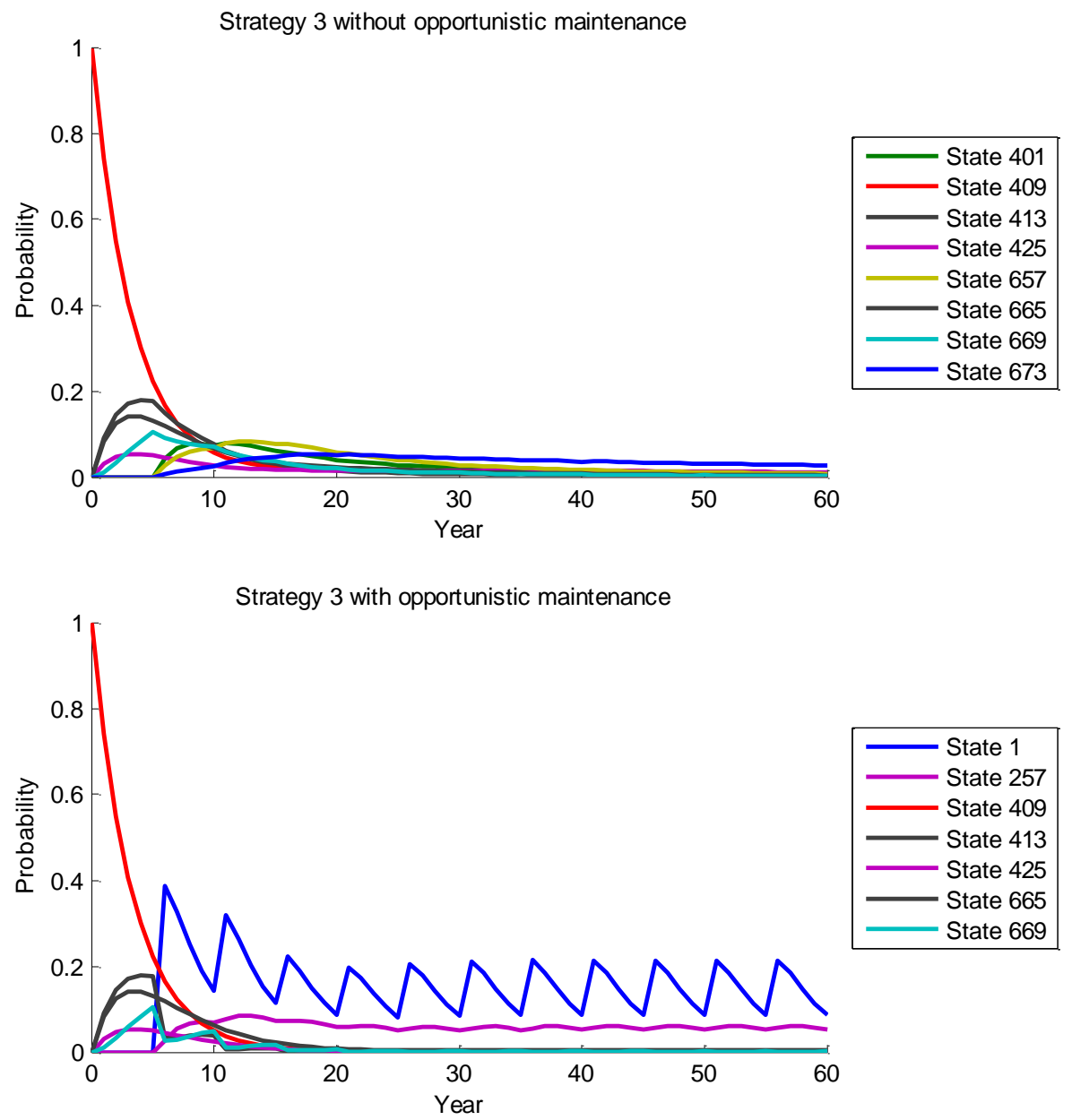

\begin{tabular}{|c|c|c|c|c|c|c|c|c|}
\hline State & DCK & MGE1 & MGI1 & MGE2 & BGL1 & BGL2 & ABT1 & ABT2 \\
\hline 1 & As new & As new & As new & As new & As new & As new & As new & As new \\
\hline 257 & Good & As new & As new & As new & As new & As new & As new & As new \\
\hline 401 & Good & Good & Poor & Good & As new & As new & As new & As new \\
\hline 409 & Good & Good & Poor & Good & Poor & Poor & As new & As new \\
\hline 413 & Good & Good & Poor & Good & V.Poor & V.Poor & As new & As new \\
\hline 425 & Good & Poor & Poor & Poor & Poor & Poor & As new & As new \\
\hline 657 & Poor & Good & Poor & Good & As new & As new & As new & As new \\
\hline 665 & Poor & Good & Poor & Good & Poor & Poor & As new & As new \\
\hline 669 & Poor & Good & Poor & Good & V.Poor & V.Poor & As new & As new \\
\hline 673 & Poor & Poor & Poor & Poor & As new & As new & As new & As new \\
\hline
\end{tabular}

Figure 4.18: Probabilities of being in different states of the bridge model under maintenance strategy 3 with and without opportunistic maintenance

\subsubsection{Analysis on a single bridge element}

As well as predicting the probability of the bridge model being in different states, analysis can be done on a single component to investigate the effect that a certain maintenance strategy will have on a particular component. This information is useful to identify critical components in the structure as well as supporting the maintenance decision making process. Figure 4.19 and Figure 4.20 plot the probability distribution for the single elements: abutment and metal bearing respectively. The plots show that 
under maintenance strategy 2 , the probability of the bearing being in either a poor or a very poor condition is about $15 \%$ whilst the probability of the abutment being in this condition is almost zero. This means that the bearing, with a faster rate of deterioration, has a higher chance of being in a poorer state, hence this component is associated with the higher risk of failure.

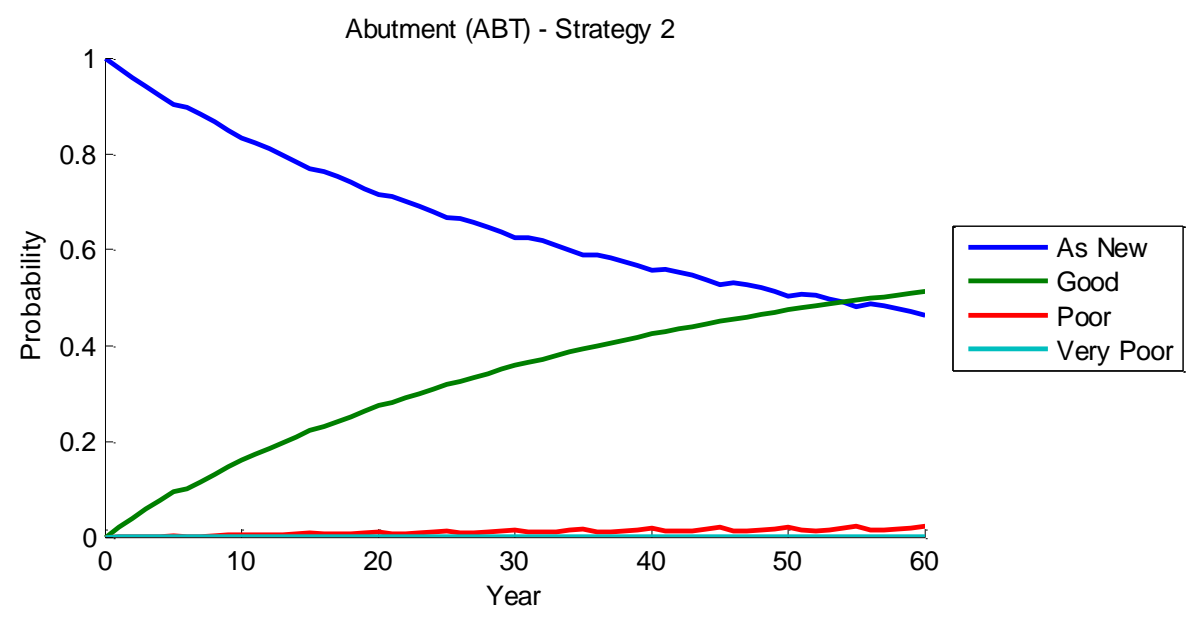

Figure 4.19: Probabilities of being in different states of the bridge abutment under maintenance strategy 2

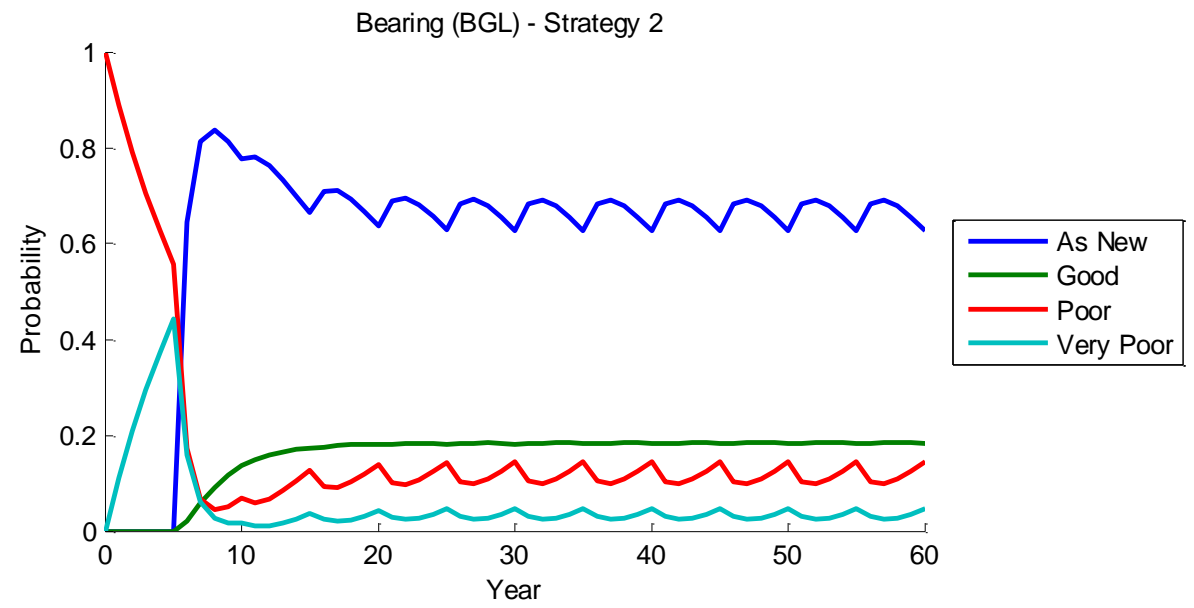

Figure 4.20: Probabilities of being in different states of the bridge bearing under maintenance strategy 2

Single element analysis reveals that the strategy applied to a bridge might not always be the best strategy for bridge elements. To look at this in more detail, assuming a scenario when the bridge is allowed up $15 \%$ of the probability of being in poor or very poor condition, single element analysis reveals that strategy 2 is appropriate when applying for the bearing as the average probability of the bearing being in poor and very poor condition is 
just less than $15 \%$. However for the abutment, applying this strategy resulting in almost zero per cent, this means that it might be unnecessary to employ strategy 2 for the abutment. Looking at Figure 4.21, where the maintenance strategy 3 is applied to the abutment, the probability of being in poor or very poor condition is within the reasonable limit $(<15 \%)$. Lowering an intervention criterion for the abutment is better in this case. The current model does not allow different strategies to be set for different elements. It is possible to integrate this option into the model by generating a different model, this will be carried out in the next chapter where a new bridge model is developed.

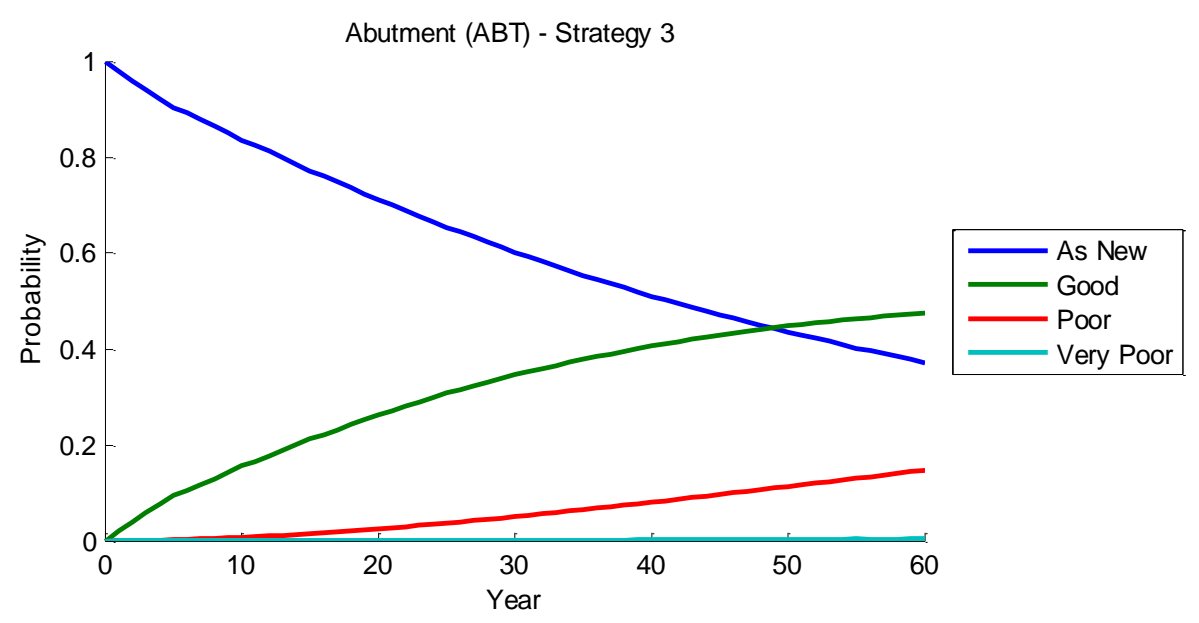

Figure 4.21: Probabilities of being in different states of the bridge abutment under maintenance strategy 3

\subsubsection{Expected total maintenance cost}

\section{Strategy 1}

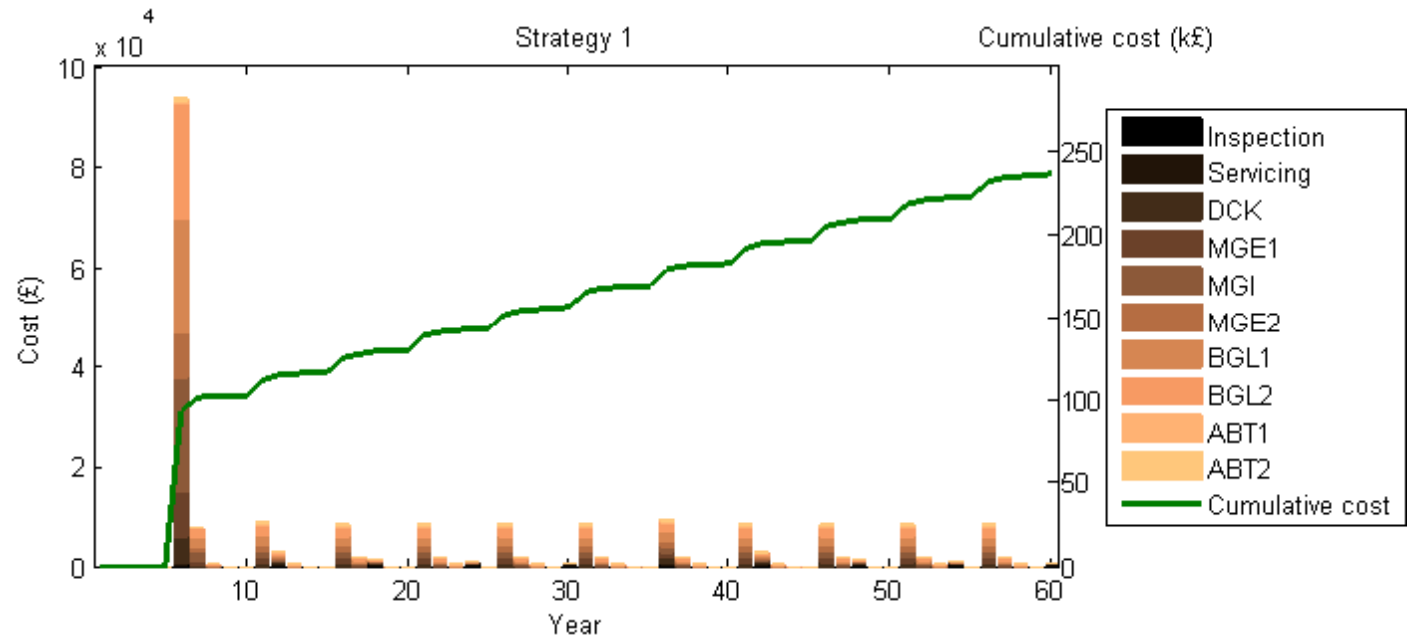

Figure 4.22: Expected maintenance cost of maintenance strategy 1 


\section{Strategy 2}

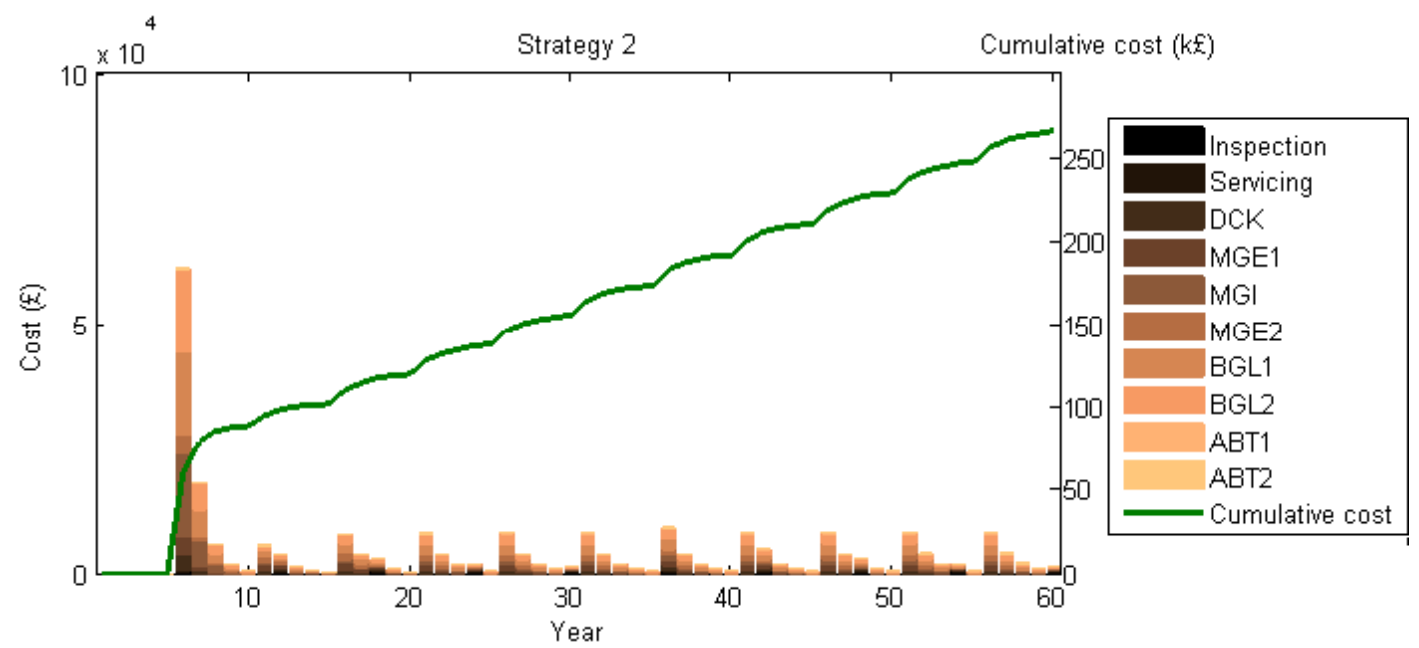

Figure 4.23: Expected maintenance cost of maintenance strategy 2

\section{Strategy 3}

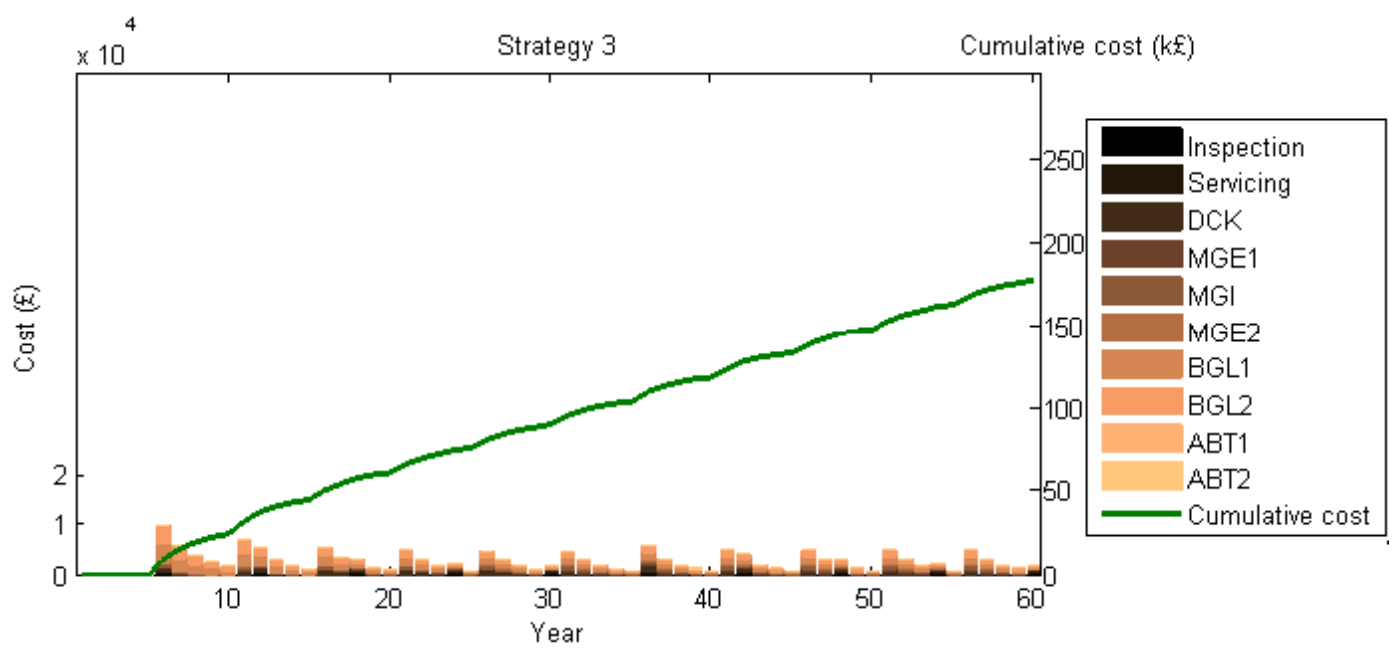

Figure 4.24: Expected maintenance cost of maintenance strategy 3

Figures 4.22-4.24 show the expected maintenance cost for maintenance strategies 1-3. These costs are estimated based on the average intervention costs as given in Chapter $3-3.6 .4$. The plots show the expected maintenance cost every year. Each column in the plots shows a stack of the maintenance cost estimated after every one year. This includes the maintenance cost for each bridge component and the recurring fixed-cost such as the inspection and servicing costs. On all the plots, the green lines show the cumulative total maintenance costs after each year until the end of the simulation time.

Figure 4.24 shows the expected maintenance costs of strategy 3 for all bridge elements and the cumulative maintenance cost over the prediction period. With this strategy, the components are left 
to deteriorate until they need replacement and since the internal main girder (MGI) and bearings (BGL) are initially in a poor condition, the probability of these components requiring repair is higher than other components resulting in a large proportion of the maintenance cost being influenced by the work done on these components.

In contrast, Strategy 1 (Figure 4.22) shows a very high peak at the start of the simulation time. This is because the strategy carries out repairs as soon as possible. Thus the components (DCK, MGs, BGLs) that are in the state where repair is possible, therefore are scheduled for repairs immediately after the first inspection. The conditions of these components are restored, resulting in lower probabilities of them being in poor conditions in the following years. This means the expected maintenance cost are also low. Very similar characteristics can be seen in Figure 4.23 for strategy 2 .

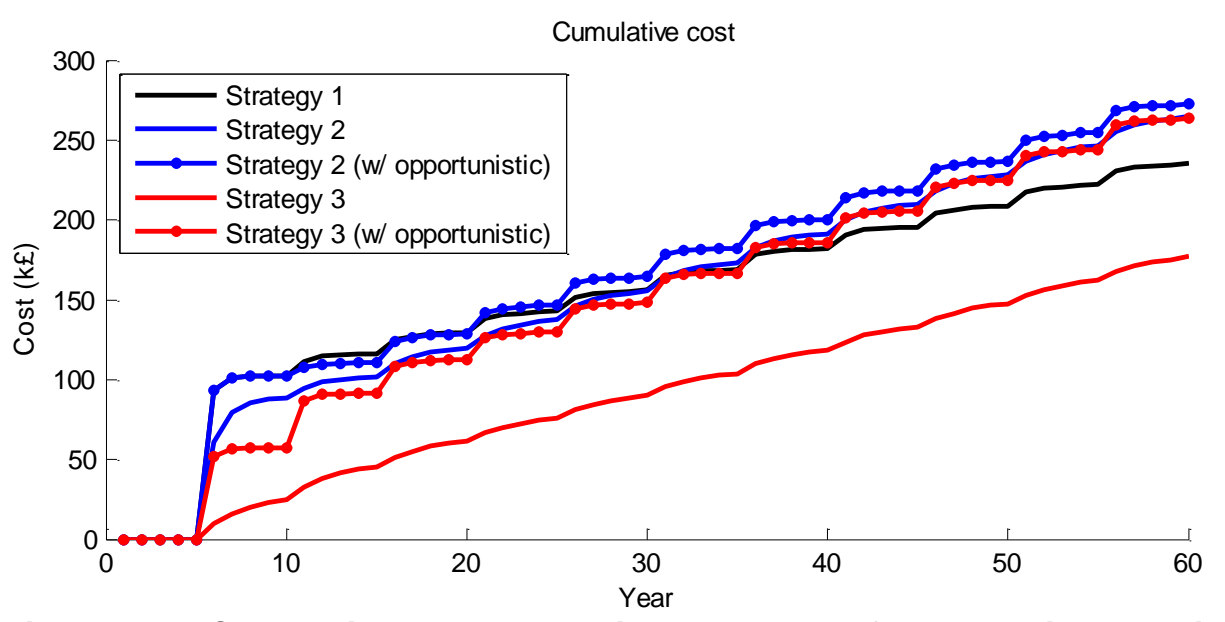

Figure 4.25: Cumulative expected maintenance cost for all repair strategies

Figure 4.25 shows the expected cumulative maintenance cost for all maintenance strategies. This is obtained by plotting the green lines in figures above together on the same graph. Note that this is the expected total cost for the whole bridge structure, the expected WLCC for each bridge component is given in Appendix $\mathrm{C}-2$. It is clear to see that following strategy 1 , because the components such as deck, girders and bearings are all in the states where the repair is necessary hence they are scheduled to be repaired immediately at the beginning of the prediction period. This results in a very high initial maintenance cost. In contrast, strategy 3 does 'minimum' work by allowing the component to deteriorate to a very poor state before an intervention, the total 
expected maintenance cost for this strategy after 60 years is around $E 177 \mathrm{k}$, which is just two thirds of what is expected from maintenance strategy 1 . It is worth noting that strategy 2 with opportunistic repair results in a similar initial cost to strategy 1 since all the bridge elements are scheduled for repair after the first inspection however, it appears that strategy 1 not only keeps the asset in better condition but also has a lower life cycle cost when comparing with strategy 2 (both with and without opportunistic maintenance).

In general, opportunistic maintenance results in a higher maintenance cost however the probability of an asset being in a better condition is higher. Depending on a particular asset, these strategies can then be applied where the trade-off between the total expected maintenance costs and condition profiles can be explored, allowing the most appropriate maintenance strategy to be selected.

\subsubsection{Inspections and servicing frequency}

In previous simulations, the inspection and servicing interval was assumed to be a 6 years interval i.e. the bridge elements are inspected and serviced every 6 years. By keeping either the inspection or servicing intervals constant and vary the other, the effects of them on the asset condition can be investigated. Figure 4.26 shows the average bridge condition at the end of 60 years lifetime against different inspection intervals ranging from 3 to 18 years. As expected, the longer time between inspection time, the higher probability of the asset being in worse condition, this is reflected in the plot as the asset condition decreases almost linearly as the inspection interval increases.

The effect of different servicing intervals on the asset condition is minimal, this is illustrated in Figure 4.27. However, it has a greater effect on the life cycle costs as this can be seen in Figure 4.28. Servicing too little or too much frequently both result in a rise in the cost. The optimum servicing frequency should be about between 4 to 8 years, if the servicing is carried out too often, the added extra cost is up to $£ 10,000$, this cost arises mainly from the cost of the servicing work itself. If the servicing is carried out not often enough, although the cost of servicing work reduces, this increases the deterioration rate of the asset resulting higher maintenance cost and contributing to a higher LCC overall. 


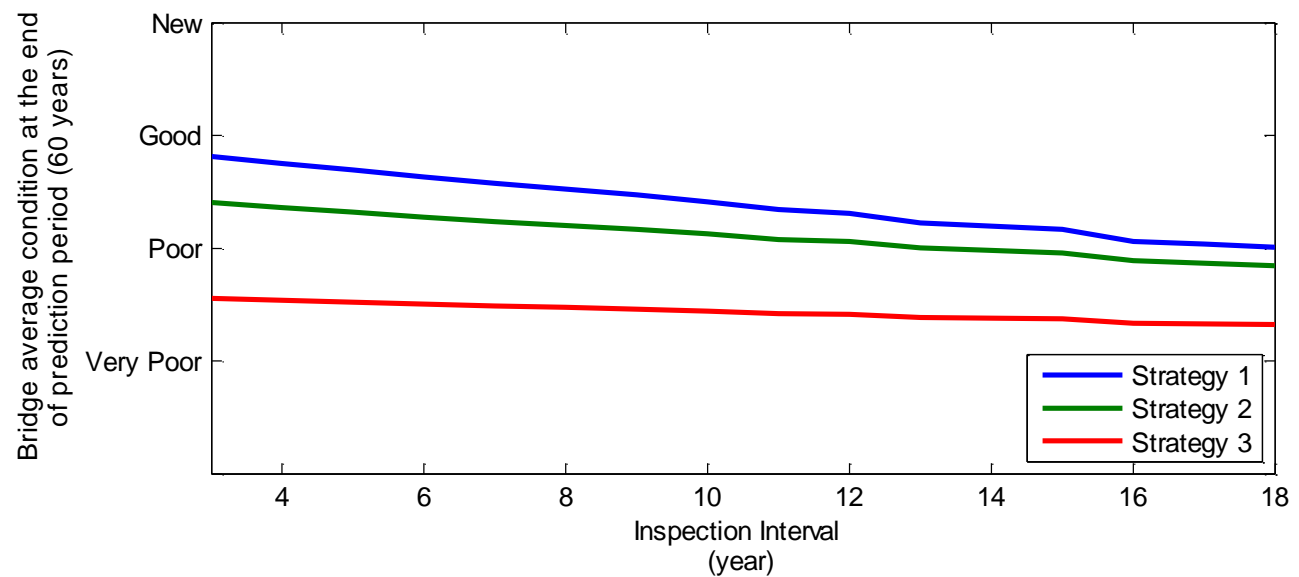

Figure 4.26: Effect of different inspection intervals on the average bridge condition

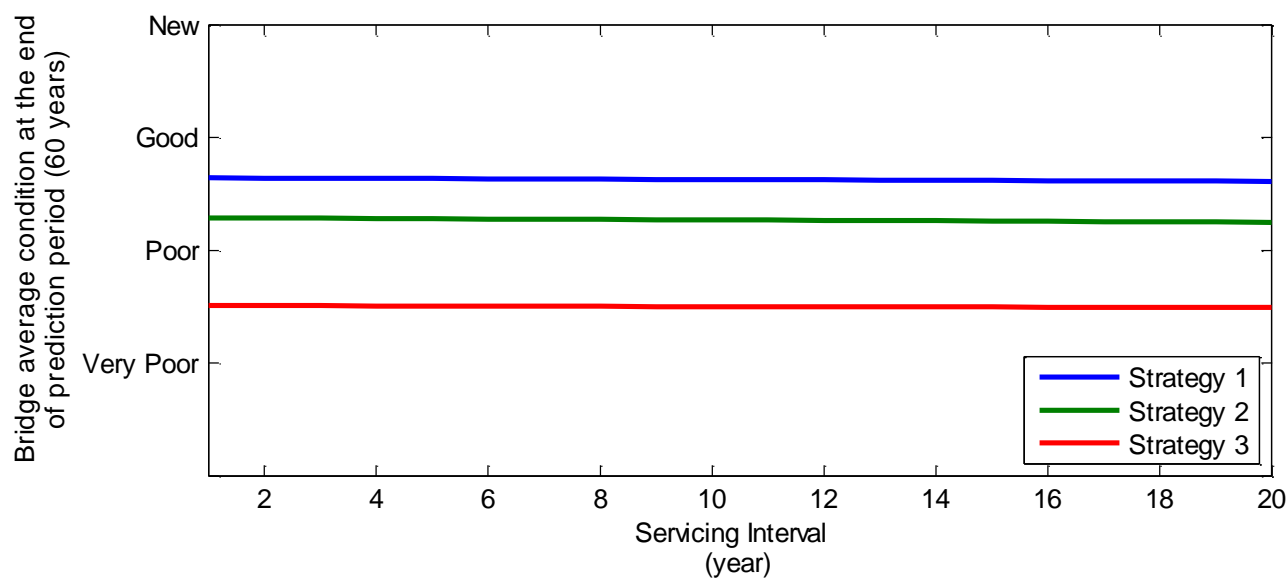

Figure 4.27: Effect of different servicing intervals on the average bridge condition

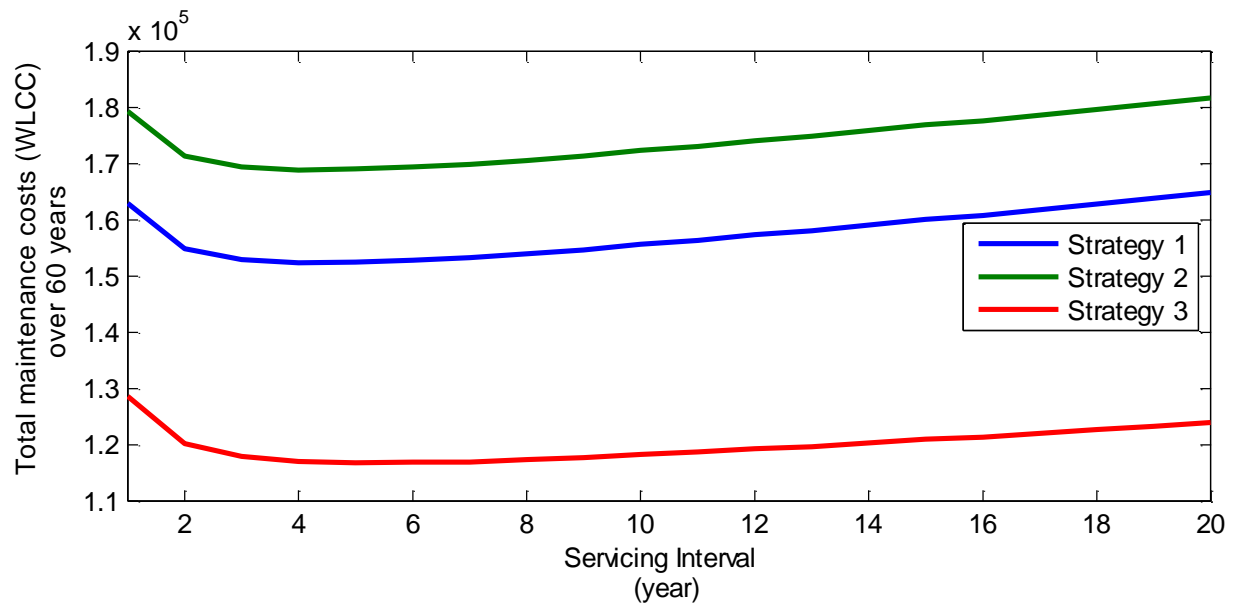

Figure 4.28: The effects of different servicing intervals on the life cycle cost of different maintenance strategies

\subsubsection{Scheduling of work - repair delay time}

By changing the scheduling time for one type of repair, the effects of delaying work can be investigated. Figure 4.29 shows the 
probability of the all bridge components being in the 'as new' state (State 1 in the Markov model) under maintenance strategy 1 with different minor repair scheduling time. As the delay time increases, the repair rate for any minor repair work in the model decreases. Slow rate of minor repairs resulting in lesser probability of all components being in 'as new' state. However, it can be seen that the effect is almost insignificant when the repair is delayed for few months and the effect is seen more clearly only when the delay time is more than 24 months.

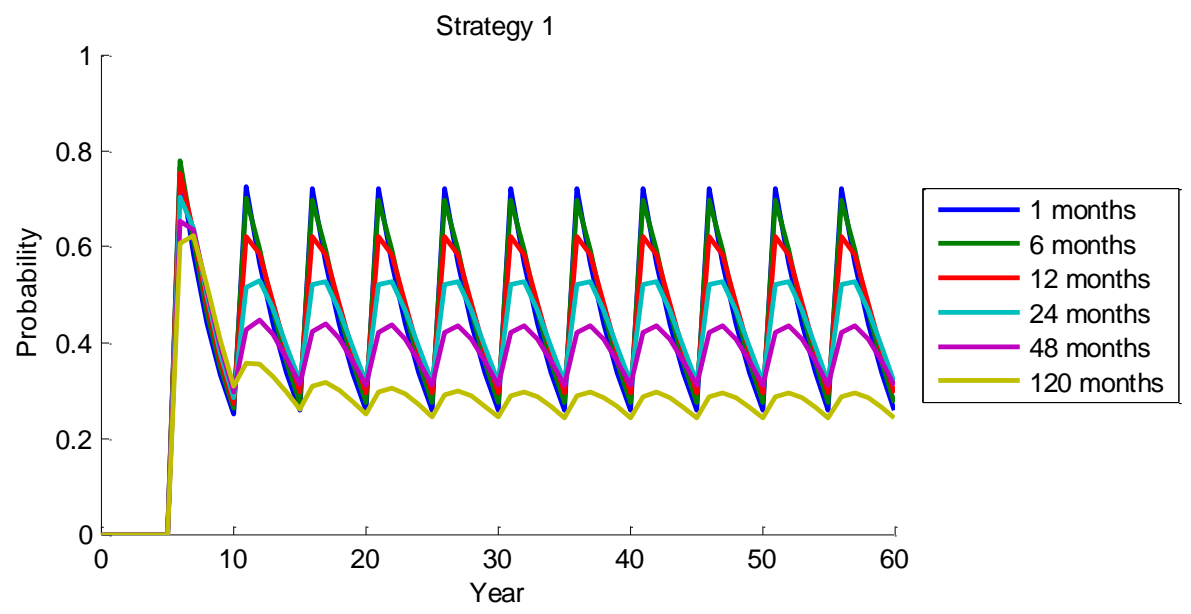

Figure 4.29: Probability of all components being in 'as new' state under maintenance strategy 1 with different delay repair time of minor repair work.

\subsection{Summary}

This chapter demonstrated a Markov modelling approach to predict the condition of individual bridge elements along with the effects that interventions will produce. The development of the bridge model was also discussed and simulation results were presented to demonstrate the capability of the model as well as the type of information the model generates that can be used to support the asset management strategy selection.

The model is capable of modelling the individual structural elements accounting for: the current (initial) condition, material type, structure type, environment, inspection intervals, servicing intervals, repair strategy and the repair scheduling (delay) times. All these parameters can be varied to allow a wide range of maintenance scenarios to be investigated. The model outputs are the probabilities of the bridge states as well as a bridge element being in different states at any given time in the future; the 
expected maintenance cost for each type of intervention for each bridge component; and the total expected maintenance expenditure - WLCC over the entire prediction period.

The advantages of the model are: the model is easy to construct and fast to run the analysis, making it easy to investigate the effect of different strategies; the model uses the degradation rates based on real historical work done data that was studied in the previous chapter; and the model is considered more detailed than previous bridge models in the literature when accounting for many factors that influence the degradation and maintenance processes.

However, it was found that in order to satisfy the requirement of the Markov modelling technique, the deterioration rates of bridge components were assumed to be constant. Furthermore, the model size increases exponentially with the number of the components making the generation, especially when generating model for repair strategy, and analysing of the model a time consuming task for model consists of more components. In the next chapter, a new bridge model is developed using a novel modelling technique that offers improvements to the Markov model as presented in this chapter. 


\section{Chapter 5 - Petri Net Bridge Model}

\subsection{Introduction}

The Petri Net (PN) was developed in 1962 by C. A. Petri (Petri, 1963). This method is increasingly being used to model dynamic systems in many engineering, science and business fields due to its flexibility (Girault and Valk, 2002). This technique has not been applied to bridge modelling before and in this chapter, the method will be explained, the development of the model and its key features will also be discussed. The advantages it can offer over the Markov approach will be illustrated. The chapter first discusses the original PN method and its development over recent years as it has been used in a wide range of reliability modelling problems. The chapter will then describe the components of the PN used in this project. A number of refinements to the traditional PN concept, to accommodate the requirement for modelling railway bridge asset management, are also explained in detail. Finally, the simulation results are presented.

\subsubsection{Original Petri Nets}

The original concept of a PN (Schneeweiss, 1999) is a directed graph with two types of nodes, called places and transitions, they are linked by arcs. A place represents a particular state or condition of a system. A token resides in a place to indicate the state of the system. A transition enables the token to move from one place to another, this models the changing state of the system. The dynamic behaviour of the system is modelled in the PN by the process of the token moving between states. 


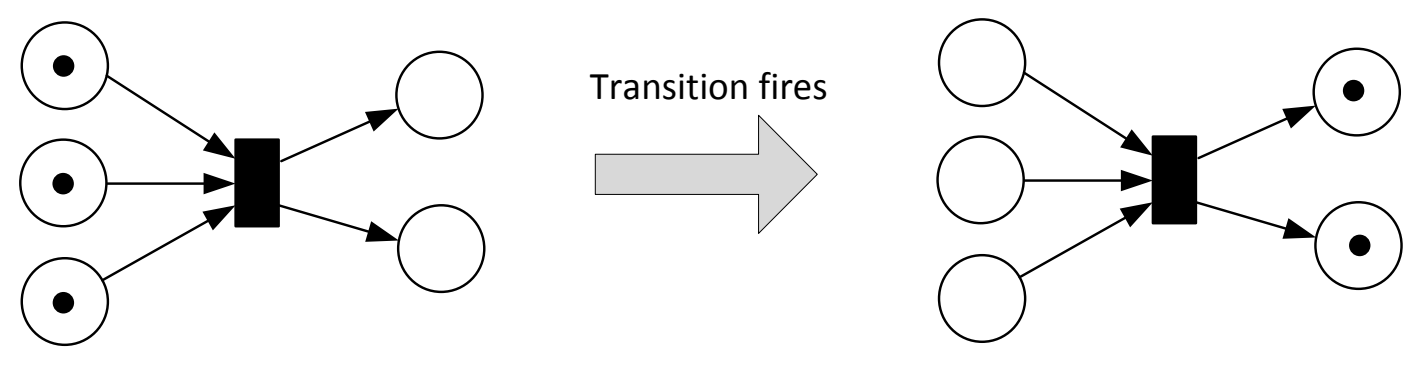

Figure 5.1: Transition fires when it is enabled

Figure 5.1 shows an example of a PN before and after a transition fires. The net contains five places (represented by 5 circles), one transition (represented by the black rectangular block), tokens are represented by black dots within the places. Places and transition are connected by arrows which indicate the direction the tokens will move. The transition is enabled when each of the places which input to the transition contain one or more tokens. After the transition fires, one token is removed from each of the input places and one token is deposited to each of the output places, the resulting marking of the PN can be seen in the figure.

\subsubsection{Variation of Petri Nets}

The increasing use of PNs has resulted in a number of variations from the original PN method. The flexibility of the PN means that special enhancements to the net can be made to adapt efficiently to a specific problem. This section discusses the variations that are available and have become an accepted feature of the PN method.

\subsubsection{Timed Petri-Nets}

In the original $\mathrm{PN}$, each transition fires as soon as they are enabled, this limits the applicability of PN on modelling timedependent systems. A number of studies have proposed a time delay associated with each transition (Holliday and Vernon, 1987, Ciardo et al., 1989, Molloy, 1982, Marsan et al., 1998). Chiola et al. (1993) developed Generalised Stochastic Petri Nets (GSPN) in which the time delay can either be deterministic i.e. a constant time delay when modelling a discrete process; or stochastic i.e. a random time sampled from statistical distributions when modelling a random process. 


\subsubsection{Extensions to Petri-Nets basic components}

1. Arc multiplicity:

Arc multiplicity (Bause and Kritzinger, 2002) is denoted by a positive integer associated to an arc (Figure 5.2). If the arc is an input arc from a place to a transition then arc multiplicity dictates the number of tokens needed for the transition to be enabled. If the arc is an output arc from a transition to a place, the arc multiplicity indicates the number of tokens that will be deposited in the output places.
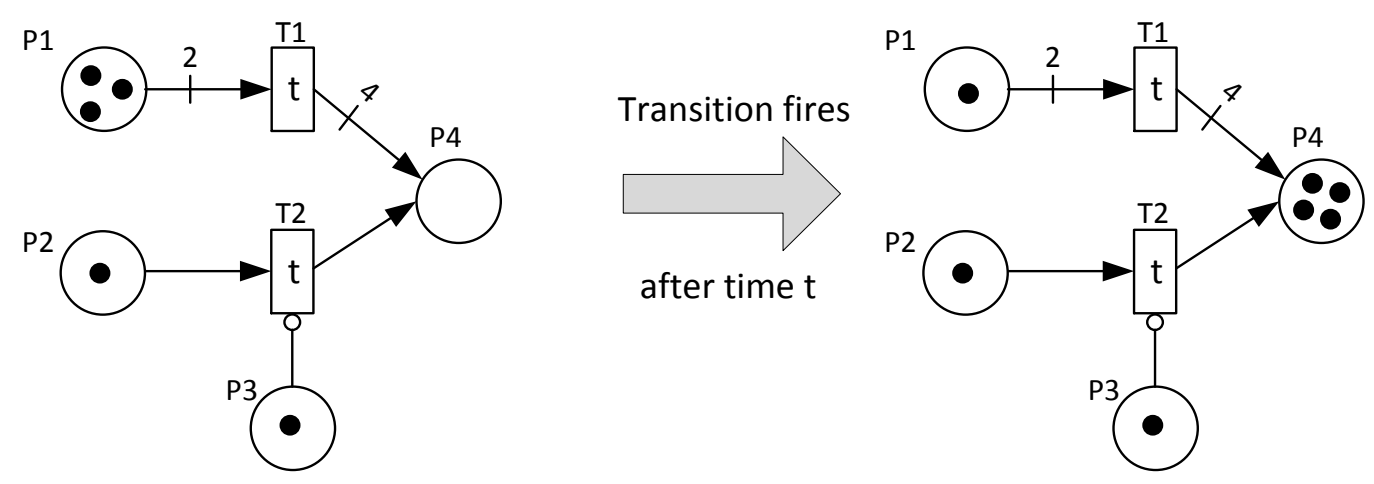

Figure 5.2: PN with arc multiplication and inhibitor arc

2. Inhibitor arc:

An inhibitor arc (Reisig and Rozenberg, 1998) can only go from a place to a transition not vice versa and is denoted as an arc with a round end (Figure 5.2). When the input place (P3 in Figure 5.2) is marked with a token, the transition (T2) is inhibited and will not fire as long as the token in place (P3) remains. This is the reason why after time $t$, the token in place $\mathrm{P} 2$ remains as the transition $\mathrm{T} 2$ is inhibited from firing by a token in place P3. The inhibitor arc may also have a multiplicity, in this case, the place must contain at least the number of tokens as indicated by the arc multiplicity for the transition to be inhibited.

\section{Reset transition}

The reset transition (Andrews, 2013), when it fires, resets the marking of specified places in the PN to some desired state. This transition has an associated list of places and number of tokens that they will contain after reset. A reset action on a network can be carried out using conventional PN features but would require a large number of transitions and places to be added which would increase the size and complexity of the model. 
4. Conditional transition

A conditional transition (Andrews, 2013) is a transition in which the delay time is sampled from different distributions depending on the number of tokens residing in a specific place in the network to which they are linked by a dashed line. Figure 5.3 shows an example of a conditional transition when it fires. The transition delay time depends on the number of tokens in place P2 that connects to transition T1. Therefore, the transition time changes each time the transition $\mathrm{T} 1$ fires since the number of tokens in place $\mathrm{P} 2$ changes after each firing.
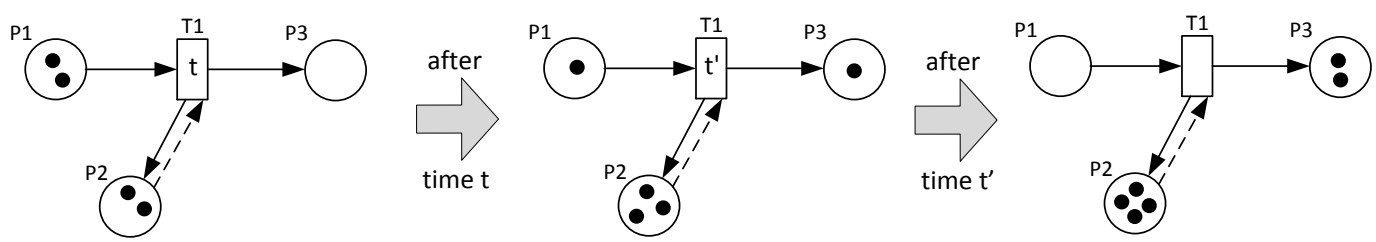

Figure 5.3: Example of a conditional transition

5. Decision making transition

When the decision making transition (Prescott DR, 2012) fires, it first takes into account the marking of certain places in the PN and uses a pre-defined rule to generate the marking of the places in the network after firing. This transition allows the decision governing which places that will be marked to be based on complex rules.

6. Substitution transition:

The substitution transition (Jensen, 1996) is effectively a sub-net of a main PN. This transition replaces a section of the net and acts as a module that can be used many times in the main net. Even though the same substitution transition can be used many times in the main net, the inputs and outputs i.e. the marking of the token inside each if these sub-nets are independent of each other.

\subsubsection{Coloured Petri-Nets}

The Coloured Petri-Net (CPN) was developed by Kurt Jensen (Jensen and Kristensen, 2009) and has been applied to several modelling problems in the field of reliability (Volovoi, 2004), software, control of systems, modelling of parallel systems (Malhotra and Trivedi, 1995). The term 'coloured' means that the tokens used in the CPN have their own colours (characteristics or labels). The colour of a token is a way of distinguishing between different tokens and carrying information through the PN. 
The basic idea of the CPN is explained in Figure 5.4. Places P1 and P2 contain a token, these tokens are of different types. The token in place $\mathrm{P} 1$ has an associated integer which has a value equal to 2 , while the token in the place P2 has a characteristic which is expressed as both an integer (value $=1$ ) and a string 'abc'. The transition $\mathrm{T} 1$ is basically a function that when enabled, fires after a delay time $\theta$. The input edge of the transition provides the inputs for the function and the output edge of the transition produces output from that function. The inputs and output are shown by the arcs that connect to the transition. When the transition fires, the tokens in place P1 and P2 are consumed and a token in place $\mathrm{P} 3$ is produce that has the properties of an integer (value $=2$ ) and a string ' $a b c$ '. The transition $\mathrm{T} 1$ also has a condition on the firing, that only when the token in place P1 has the integer value of greater than 1 then the transition may be enabled.
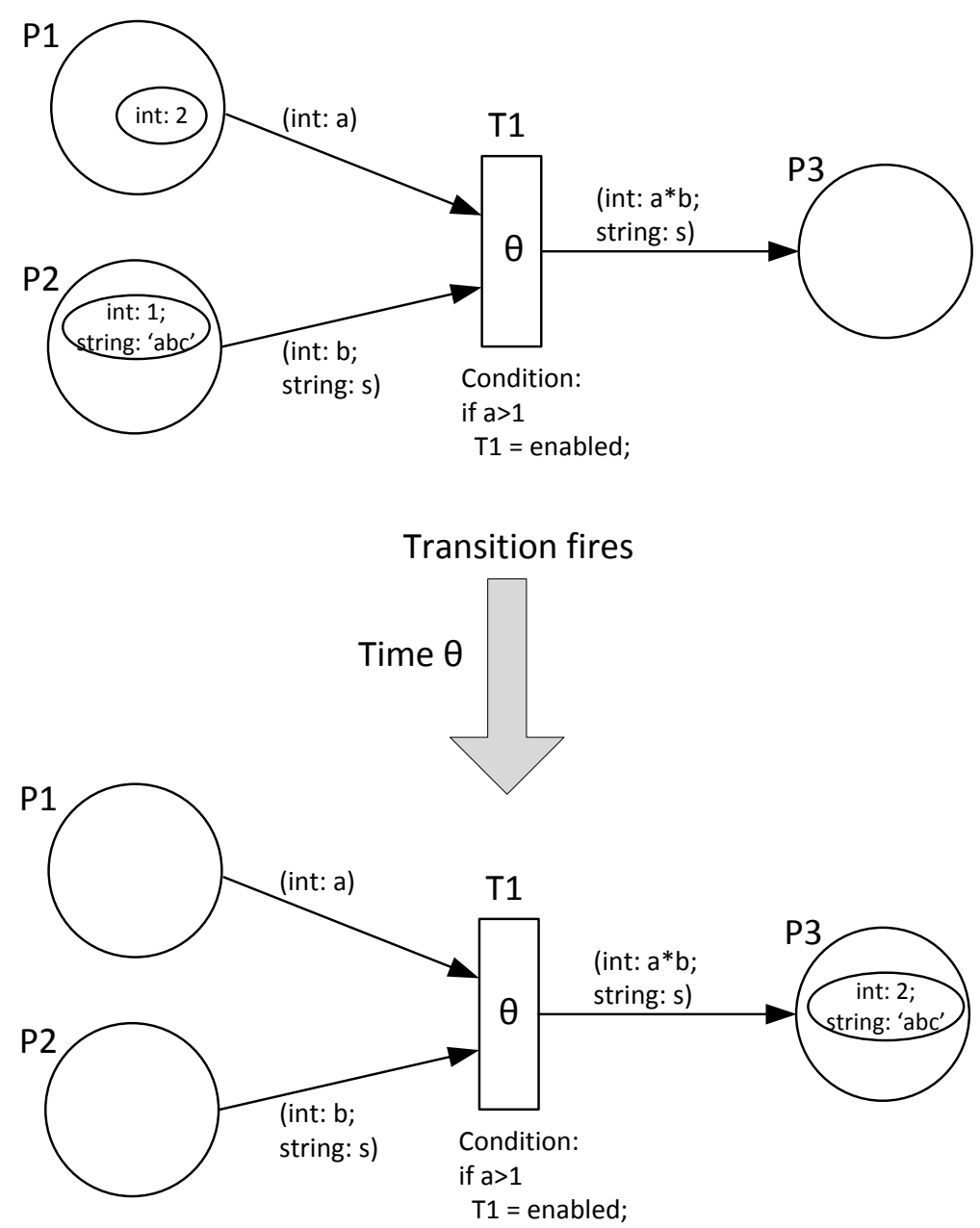

Figure 5.4: Coloured Petri Nets 
In the CPN, the basic concept of the original PN is preserved. The difference in the CPN is that, since the transition is now a function, it is possible for the characteristics of the token to change once it passes through a transition. Whereas, in the traditional PN, the tokens are simply consumed at the input and reproduced at the output of a transition. The flexibility and advantage of the CPN comes from the ability to transform part of the PN into a function. Thus, the flexibility of the PN can be further enhanced by modifying part of the net (such as the arc) to contain a function that effects the token in the desired way.

\subsection{Model definitions}

The PN used in this project can be regarded as an extension to the traditional PN. The most important characteristic in the PN used in this project is that each token is treated separately and they each contain a set of properties that is unique to each bridge component being modelled. Part of the net is modified to suit the problem in bridge asset management. These improvisations are discussed below.

\subsubsection{Token}

Each token used in the PN represents a particular bridge element. A token carries a set of properties with it. Each token is unique with an associated ID. The properties stored inside a token are as follows:
a. Token ID
b. Component type/name
c. Component material
d. Coating condition
e. Environmental factor
f. Repair history (all repairs have happened and the time at which they were carried out)

\subsubsection{Place}

A place denoted by a circle represents the state of the bridge component.

\subsubsection{Transition}

a) Conditional transition 
A conditional transition is a stochastic time delay transition, the time is sampled from a specific distribution. Depending on the token that is going to be fired through a transition, an appropriate distribution is selected. Figure 5.5 shows an example a PN with two places $\mathrm{P} 1$ and $\mathrm{P} 2$, a conditional transition $\mathrm{T} 1$ and two unique tokens (black dot and white circle). After time $a$, the white token is fired and after time $b$, the dark token is fired. These times are sampled from different distributions as the tokens represents different items. This is different to conventional PN, as the transition time would be sampled from the same distribution. The conditional transition represented by a white rectangular block.

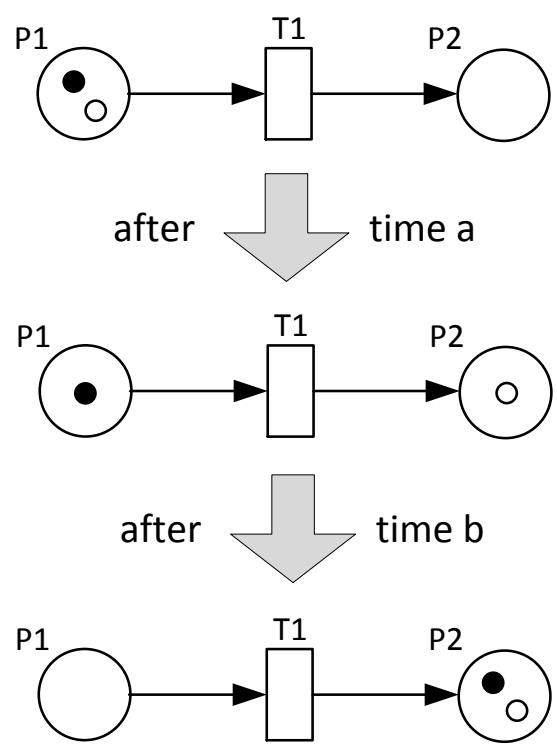

Figure 5.5: Conditional transition

b) Periodical transition

The periodical transition is a type of transition that only fires when the system is at a specified time, i.e. the transition delay time depends on the periodical time when the transition is allowed to fire and is given in the equation below:

Transition delay time $=$

Time at which the transition is allowed to fire - System time

The periodical transition is represented by a solid dark rectangular block. In this project, this transition is used to model the inspection process where the condition of the bridge element is revealed after a specified time interval. It is also used to model the maintenance schedule for a track so the repair can only happen at the time the possession is taken. 


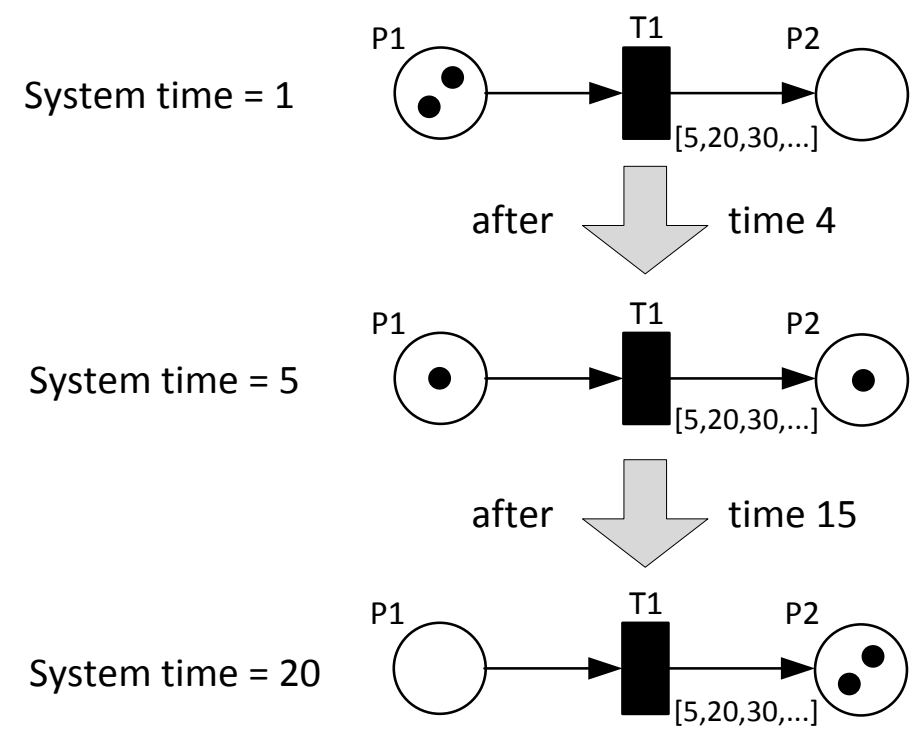

Figure 5.6: Periodical transition

Figure 5.6 shows the firing sequence of a periodical transition, T1. The time at which the transition can fire is when the system time is set at $[5,10,30 \ldots]$. The transition is enabled when the system time is 1 , however since the transition is only allowed to fire when the system time is 5 , the firing of the token is delayed by $5-1=$ 4 time units. After the first firing, a token is transferred from place $\mathrm{P} 1$ to $\mathrm{P} 2$. Now the system time is at 5 , the next firing can only happen when the system time is at 20 . Thus the transition time is delayed by 15 time unit.

\subsubsection{Firing rules and conflicts}

The firing and associated time delay are used as in the original PN method. However with the use of a unique token and a conditional transition, there are certain circumstances in which the firing logic must be defined. This section discusses all the rules employed in the PN and the firing conflicts.

\section{Rules}

- Each transition and place has got a unique ID they are used for identification when constructing a net.

- Token has a unique ID and carries with it a list of properties.

- For the transition to be enabled, it requires the same type of token to be presented at every input place. Figure 5.7 shows the marking of the net that contains three different tokens (black, shade and white token) before and after the transition fires. The marking of the black token in the place 
P3 inhibits the firing of black tokens in place P1 and P2 however this does not inhibit the firing of the shaded and white tokens. After the transition fires, only the shaded tokens are removed from place $\mathrm{P} 1$ and $\mathrm{P} 2$ and deposited in place P4. The white token stays in the place P1 since it is required for another white token to be in the place $\mathrm{P} 2$ for the transition of white token to be enabled.
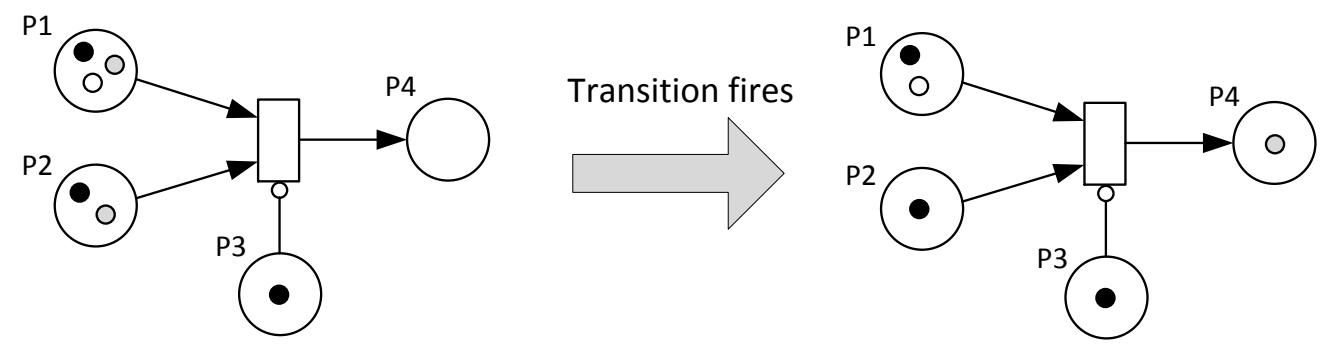

Figure 5.7: Firing rule with different tokens

\section{Conflicts}

If two or more transitions are enabled from a common place, the transition with the lowest time delay will fire first. However, since the delay times are sampled from distributions, there might be a case that the delay times are exactly the same. In this case, though unlikely, the transitions will both try to take the token from the common place as demonstrated in Figure 5.8. By introducing the transition priority, the transition with higher priority will fire first. In the $\mathrm{PN}$ in this project, the transition priority is introduced simply by the ID numbers of the transitions. The transition with the lower ID number will have higher priority e.g. transition T1 would be prioritised over transition T2 if they both have the same firing time.
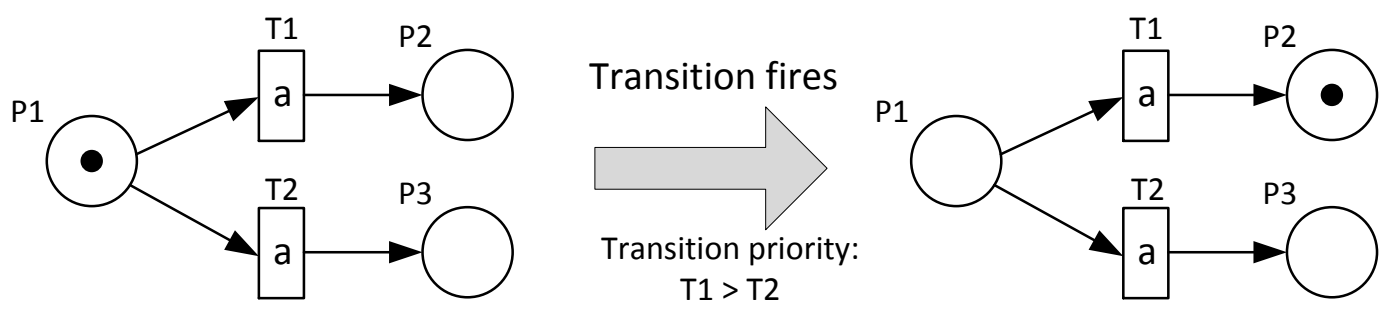

Figure 5.8: Transition priority

\subsubsection{Transition rates between condition states}

Chapter 3 - section 3.6.1 presented the distributions of the lifetime of the components i.e. the time when the component reaches a certain condition from the 'as good as new' condition 
(illustrated in Figure 5.9). However the PN model requires the transition rates between each of the four states used i.e. distribution of times moving from the 'as good as new' state to a good state, from a good state to a poor state and from a poor state to a very poor state. This is demonstrated in Figure 5.10.

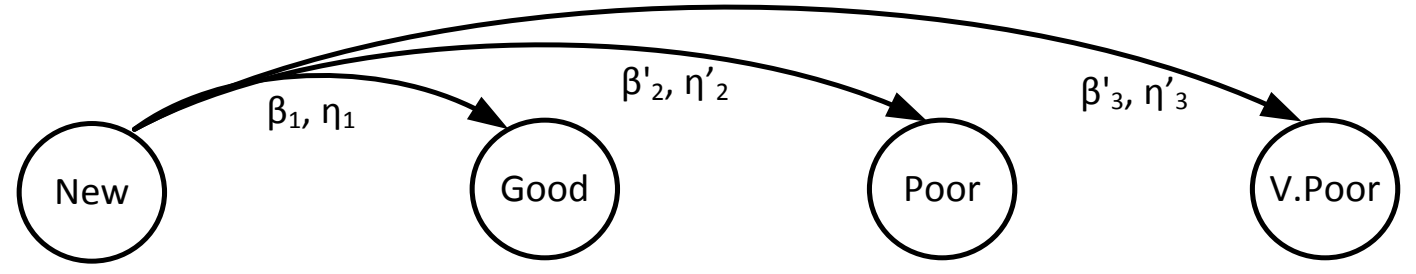

Figure 5.9: Deterioration process from the 'as new' state

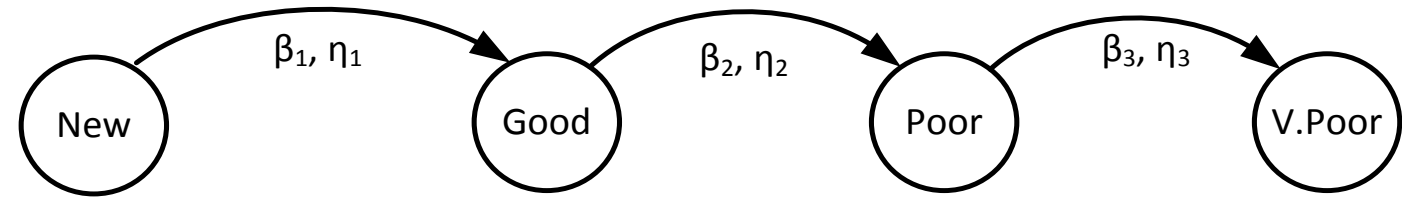

Figure 5.10: Deterioration process between each condition states

This is called convolution transitions, as discussed in Andrews (2013). The convolution transition can be estimated by the mathematical process, however in this project, the estimation of this convolution transition is by simulation. This is done prior to the running of the model simulation.

Figure 5.11 below shows the Petri-Net model to model the degradation process from a new state (P1) to a poor state (P3). The two processes are the same however in the second process an extra state is added representing the good state (P2) that the component must pass through in order to reach the poor state. We have a Weibull distribution for the time it takes an element to degrade from the new state to the poor state (T1) and from the new state to the good state (T2) with parameters $\beta_{1}, \eta_{1}$ and $\beta_{2}, \eta_{2}$ respectively. The distribution of times from the good to the poor state (T3) has parameters $\beta_{3}, \eta_{3}$. These can be estimated by simulating both processes for a given time period and the distribution that would generate similar outcomes for both processes found. Given a range for Weibull parameters, a geneticalgorithm optimisation technique was used to search for the best values of $\beta_{3}, \eta_{3}$ that minimises the number of tokens resulting in 
the poor state after a certain time, after a certain number of simulations.
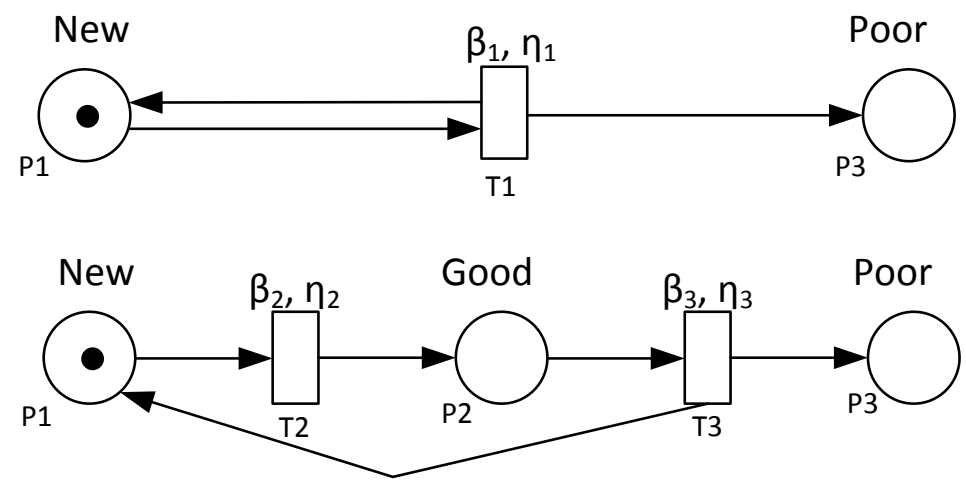

Figure 5.11: Convolution transition between condition states

The optimisation search is explained in detail as follows:

1. For a range of values of $\beta_{3}, \eta_{3}$, the Petri-net model given in Figure 5.11 is simulated for a given time period.

2. At the end of the simulation time, the model stops and the difference in the number of tokens residing in place P3 of both processes is calculated.

3. This simulation is repeated for a number of times and the mean difference in the number of tokens for the two processes is calculated.

4. The Genetic-algorithm optimisation technique is applied to find the optimum values of $\beta_{3}, \eta_{3}$ so that the mean difference calculated at the step above is minimised.

It is recognised that this is a practical approximation as given $\mathrm{T} 1$ and T2 are Weibull distributions, T3 will only be approximated by a Weibull distribution.

\subsection{Petri-Net Bridge Model}

This section describes the construction of the Petri-Net bridge model.

\subsubsection{Degradation process}

It is assumed that any bridge component can be in one of four conditions as discussed in Chapter 3 . The component starts in the new condition and degrades to a good condition which would require a minor repair. Further deterioration leads to the poor and 
very poor conditions where the component requires major repair and complete renewal to return it to 'as new' respectively. The degradation process can be modelled using the net as demonstrated in Figure 5.12. Places $\mathrm{P} 1$ to $\mathrm{P} 4$ represent the four condition states and the transitions between these states are represented by transition T1 to T3. The transition times are governed by the Weibull distributions obtained as described in Chapter 3 - 3.6.1.

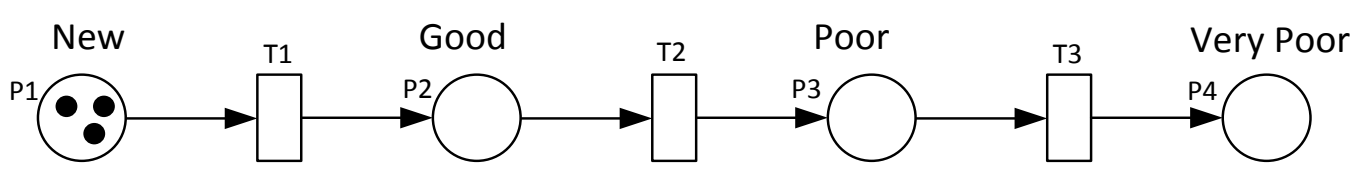

Figure 5.12: Petri-Net models the degradation process

\subsubsection{Inspection process}

All bridges and their components are inspected after a certain period of time when the true condition of the component is revealed. When the bridge component condition is inspected then the appropriate type of maintenance work can be applied. The inspection process is represented by the periodical transitions T4, T5 and T6 as illustrated in Figure 5.13. The inspection time is set using these transitions. Assuming the inspection time is set to inspect at every $\theta$ time units and the component has reached good condition (P2), there are two possible transitions from this state which are represented by transitions $\mathrm{T} 2$ and $\mathrm{T} 4$. If the transition T4 is fired first, the token is transferred to place P5 which means the component is now been inspected and the condition is revealed to be in the good condition. However if the transition T5 is fired first, the token is transferred to place P3 which means the component has degraded to the poor condition (P3) before it was inspected. The transition time T2 is sampled from the distribution of times as described in Chapter 3 - 3.6.1. The transition time $\mathrm{T} 4$ is the time to inspection i.e. the time between which the token arrives at $\mathrm{P} 2$ and the time when the component is next inspected $\theta, 2 \theta, 3 \theta \ldots n \theta$. Similarly, the inspection transition T5 and T6 are used to inspect when the component reaches the poor and very poor states. 


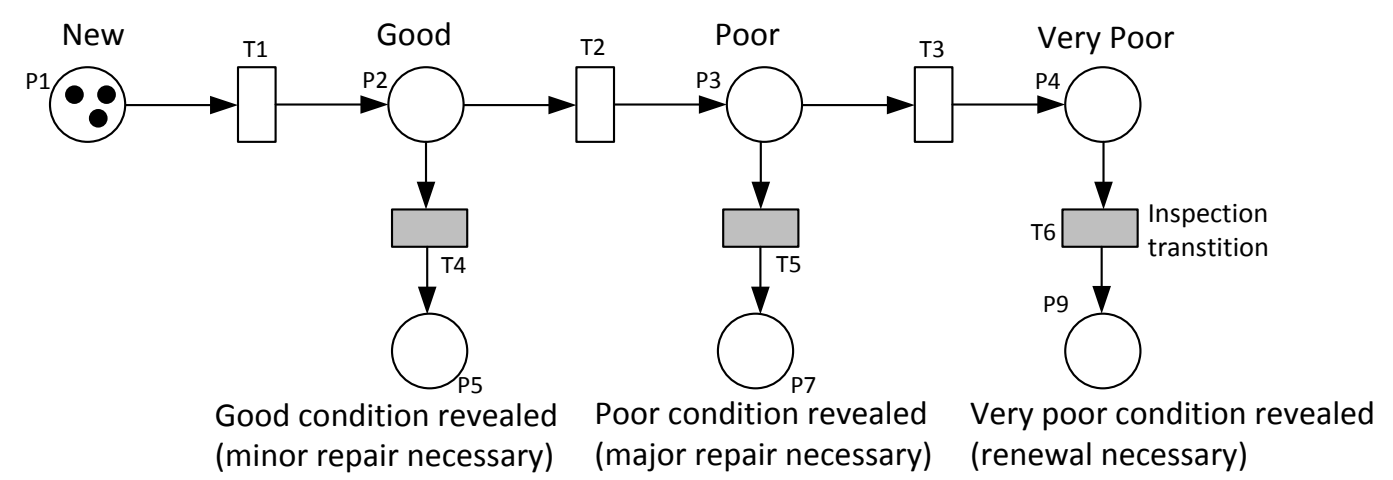

Figure 5.13: Inspection process

\subsubsection{Maintenance schedule and delay repair process}

When the condition of the bridge element is identified, a certain type of maintenance action can be triggered to restore the element condition to as good as new. The maintenance process does not usually happen immediately and often has an associated delay time, the repair delay time depends not only on the bridge management authority but also (if necessary) on the maintenance schedule of the line as some interventions can only be carried out when there are no trains running, therefore maintenance actions are usually planned. The maintenance schedule is often set by the authority considering the maintenance of different parts on the railway network (e.g. route criticality, the condition of railway line, etc.). Figure 5.14 demonstrates an example when the bridge deck needs local patching whilst the main girder needs major plating. There is no delay on girder plating hence it is repaired at the first maintenance block available. Deck patching, however, was delayed and the repair happens at the first available block of maintenance schedule after the delaying period.

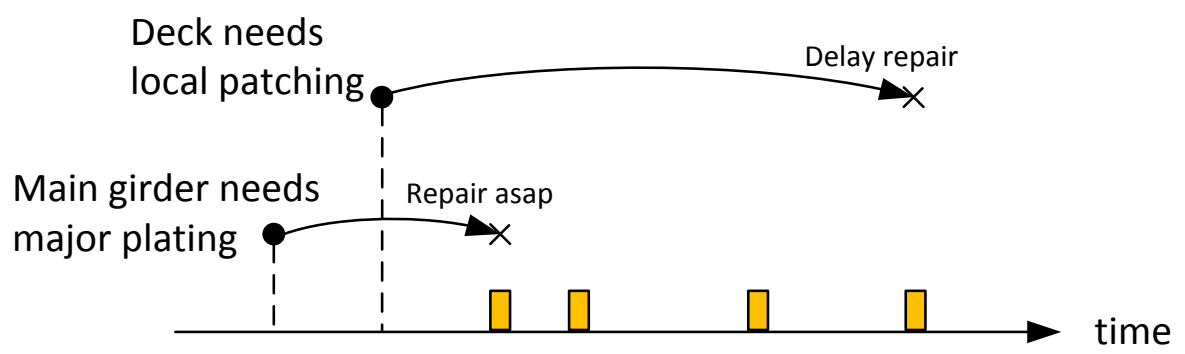

- The point when the component requires intervention

$x$ The component is being repaired

Maintenance schedule block at which repair can only happen

Figure 5.14: Delay repair and maintenance schedule 
The equivalent Petri-Net to model this process is shown in Figure 5.15. Places $P 1$ and $P 2$ are marked to show that the deck requires concrete patching, and the girder requires major plating. Place P3 represents the place where the interventions are queued up and ready to be implemented at the next available block of maintenance schedule. The transition from P1 to P3 is governed by the transition $T 1$, a delay time, $t 1$, is set on this transition to represent the delay of concrete patching. In contrast, the transition from P2 to P3 represented by the transition T2 has the delay time of 0 . This means that when the place P2 is marked, the token enables the transition $\mathrm{T} 2$ and this transition immediately fires which then removes the token in place P2 and mark the token in place P3. This also means that the intervention on the main girder would be queued up in the job list instantly and the repair will happen at the next available maintenance slot. Transition T3 is a periodical transition, as described earlier, and contains the maintenance schedule which governs the time at which the token in place P3 is fired.

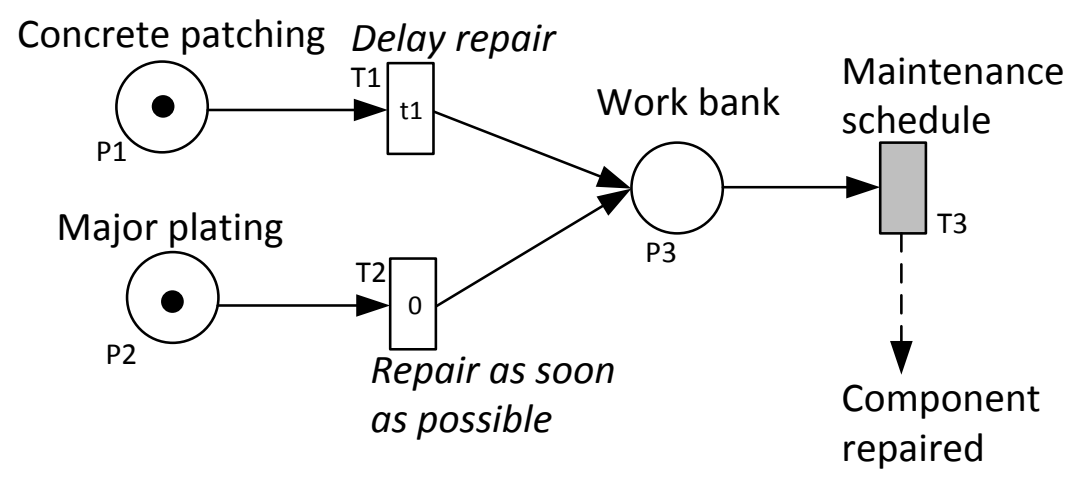

Figure 5.15: Petri-Net models the delay repair and maintenance schedule

So far, it has been explained how the maintenance delay process can be modelled in the Petri-Net, applying this to the states where the bridge component condition is revealed after inspection and the maintenance is necessary (Place P5, P7, P9 in Figure 5.13), the Petri-Net models the maintenance process for bridge elements can be seen in Figure 5.16. Transitions T7, T8 and T9 represent the repair delay time for each type of repair (minor repair, major repair and renewal respectively).

Place P10 is effectively the job list that is to be carried out at the next schedule block available which is set by the transition T10. Following a repair, the token is transferred to place P1 implying 
the condition of the element is restored to the as good as new condition.

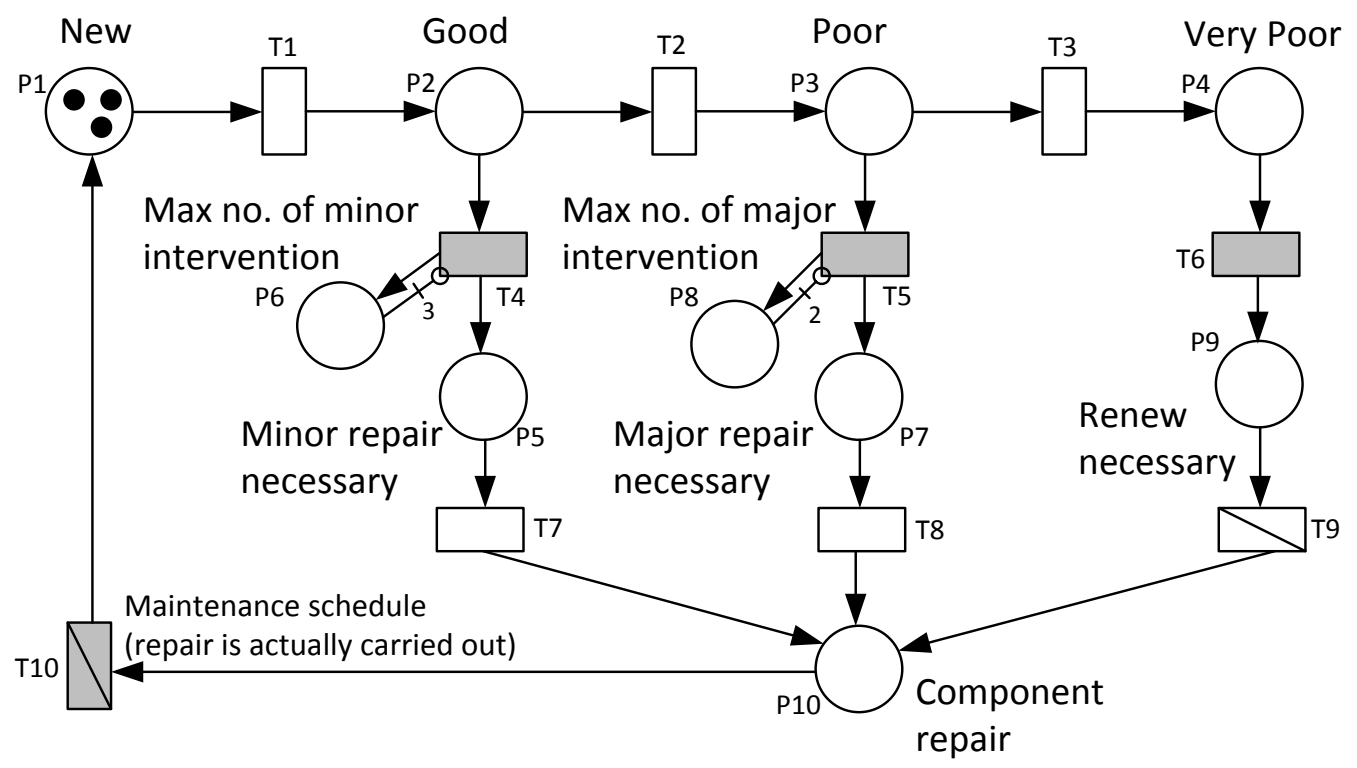

Figure 5.16: Intervention and repair delay process

It is important to realise that certain types of intervention are ineffective after being carried out a certain number of times. This can be modelled by introducing the places (P6 and P8 in Figure 5.16) recording the number of times a certain type of maintenance was used and inhibit the repair process once the maximum number of that type of intervention is reached. Assuming the bridge element is in a good condition (P2) when it is inspected, this means the transition T4 fires and the token is now removed from $\mathrm{P} 2$ and marked in place P5 and P6. The number of tokens in place P6 indicates the number of minor repairs that have happened over the component life time. Place P6 connects with transition T4 by an inhibitor arc with the multiplicity of 3 . Thus when there are three tokens of the same type in place P6, the transition T4 will be inhibited. This means that once three minor interventions were carried out, minor intervention is not possible anymore. Similarly, place P8 records the number of major repairs that have happened. The maximum number of major intervention possible on a bridge component is set by the multiplicity of the inhibitor arc connects place P8 and transition T5.

Note that following a component replacement (place P9 component renewal), the history of the number of minor and major interventions recorded in places P6 and P8 should be 
cleared as this does not relate to the newly replaced component. This is implemented in the model by using the reset transition T9. When this transition fires, the tokens in places P6 and P8 are cleared. It can be seen that transition $\mathrm{T} 10$ is also a reset transition, when this transition fires, it resets the condition of the coating, paint, protective layer etc. of the bridge element. This will be explained in more detail in the next section.

\subsubsection{Opportunistic maintenance}

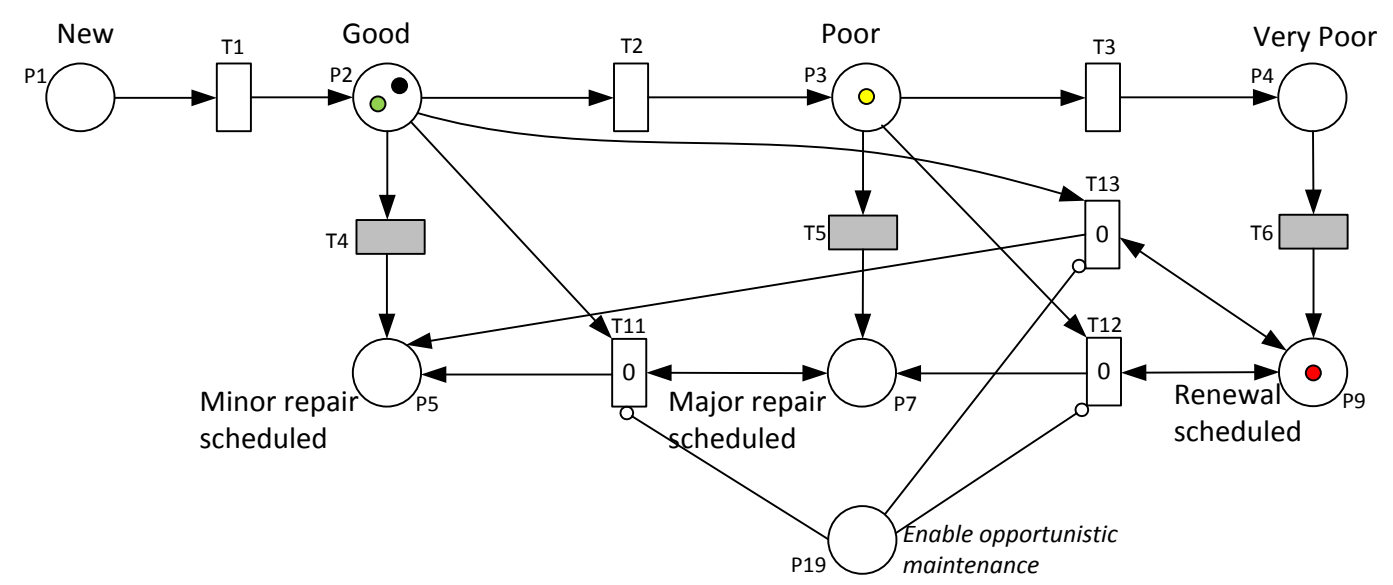

Figure 5.17: Petri-net module representing opportunistic maintenance

Figure 5.17 illustrates the PN representation to model the opportunistic maintenance. There are black, green, yellow and red tokens that model four bridge main girders. The red token is in the place P9 where it is scheduled for a renewal, this means that one girder is in the poor condition and after the inspection where its condition is revealed, the girder will be replaced. The other girders represented by the black, green and yellow tokens are currently in the good and poor states. Opportunistic maintenance considers carrying out minor and major repairs on the black, green and yellow tokens. This is modelled by introducing transitions $\mathrm{T} 12$ and $\mathrm{T} 13$. The red and yellow tokens enable transition T12 and after the transition fires, the yellow token is transferred to place P7 where it is scheduled for a major repair. The red token is cleared from place P9 when the transition fires but is then deposited back in place P9 immediately after the firing as indicating by the double ended arc connecting place P9 to transition T12. Similarly, the red, black and green tokens now enable the transition T13 which transfers the green and the black token into place P5 which indicates a minor repair will be carried 
out. Finally, transition T11 is added to model the opportunistic minor repair when there is a component undergoes major repair.

Transitions $\mathrm{T} 11, \mathrm{~T} 12$ and $\mathrm{T} 13$ are conditional transitions with zero time delay as noted in the figure. The zero firing time is to ensure that opportunistic maintenance is implemented immediately after the scheduling of an intervention. It is also important to know that, the conditional transition warrants correct deposition of tokens after firing. In particular, the red token is deposited in place P9 after firing, not in places P7 or P5. Also the green and black tokens are transferred to place P5, the yellow token is deposited to place P7. Moreover, opportunistic maintenance only considers similar components. Thus, transitions T11, T12 and T13 are enabled only when the tokens model the same type of component. For example, if the yellow token models an abutment, the transition $\mathrm{T} 12$ is not enabled. In this chapter, opportunistic maintenance is considered on components of the same type such as: deck, bearing, girder, and abutment. The nature of the conditional transition allows all of the mentioned rules to be implemented quite easily.

Figure 5.17 also shows place P19 which connects to transition T11, T12 and T13 by inhibitor arcs. Tokens can be added to this place to disable the opportunistic maintenance option in the model. If there is no token, then the opportunistic maintenance is considered.

\subsubsection{Modelling of the protective layer of metallic elements}

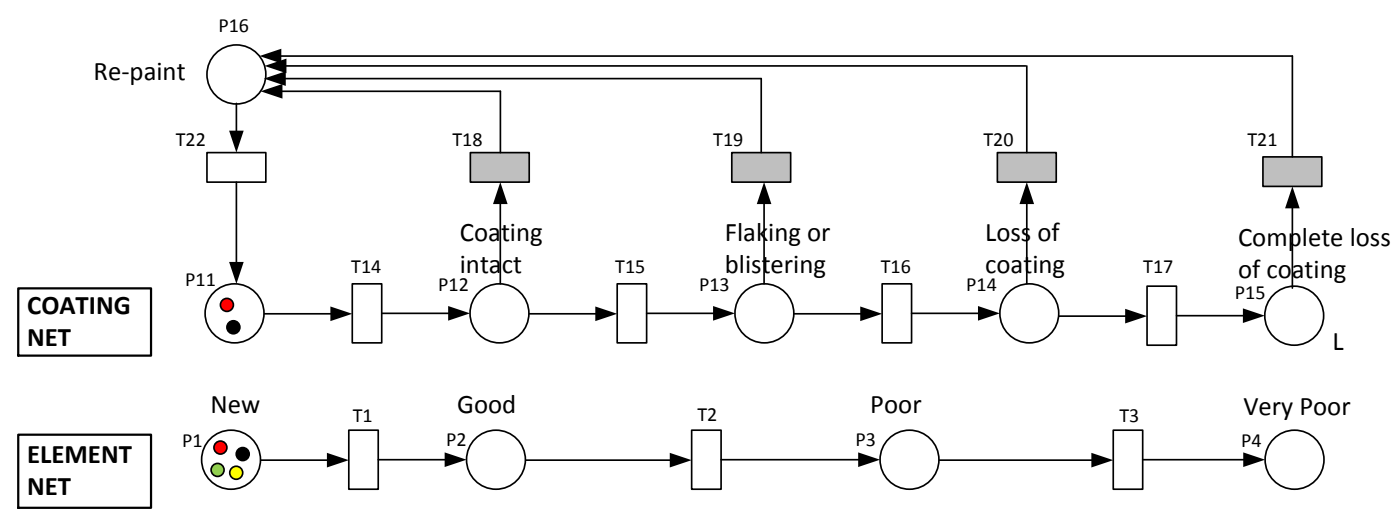

Figure 5.18: Petri-Net models metal element include the condition of coatings

The degradation rate of the metal element depends on the condition of the protective coating. Appendix D-1 reports the study of the degradation of the protective coating and its effect 
on the deterioration rate of its protected metal. It is possible to capture and model this effect in the PN bridge model. Figure 5.18 shows two separate Petri-nets, the top net models the condition of the coating and the bottom net models the condition of the metal element itself. The bottom net should extend to be the same as the net presented in Figure 5.16, however, only part of the net is illustrated here. There are five coating conditions which are represented by places $\mathrm{P} 11$ to $\mathrm{P} 15$. The transitions between these conditions are represented by transitions T14 to T17. Transitions T18 to T21 are periodical transitions which are used to model the inspection process where the condition of the coating is revealed following an inspection. It is assumed that if the coating condition is revealed to be in the state where there is some loss (P14) or a complete loss of coating (P15) then the component will be re-painted (P16) and the condition of the coating will be restored to the new condition.

Even though the two nets are separated however the modelling of the deterioration processes of the element and its coating are dependent. This is modelled by connecting the token in both nets through their characteristics. In the net PN1, the position of the token updates that token property. This property i.e. the current condition of the coating is therefore also captured in the token in the net PN2. Based on the property of the token, an appropriate degradation rate is chosen to model the deterioration of the metal element in the net PN2.

As mentioned previously, the transition T10 in the net PN2 is a reset transition (transition T10 can be seen in Figure 5.16). When this transition fires, it resets the net PN1 by removing the token in any place in the net and marks the token in place P11. This reset action in the model implies that when the repair happens on the metal element, the coating of that element is also restored to new condition.

\subsubsection{Bridge model}

\subsubsection{Intervention options}

The intervention strategy can be set by placing tokens into places $\mathrm{P} 17$ and P18 in the Petri-Net as demonstrated in Figure 5.19. These places inhibit the transitions T4 and T5, thus disable a certain type of repairs for a bridge component. In this way the intervention strategy can be applied differently on each bridge 
component. The green tokens in places $\mathrm{P} 17$ and $\mathrm{P} 18$ represent the scenario where only renewal is possible for the bridge element modelled by the green token. The red token in place P17 represents the scenario where the component is allowed to deteriorate past the good state and interventions only happen at the major intervention level. For the component represented by the black token, the component is repaired as soon as it is in the state where intervention is possible.

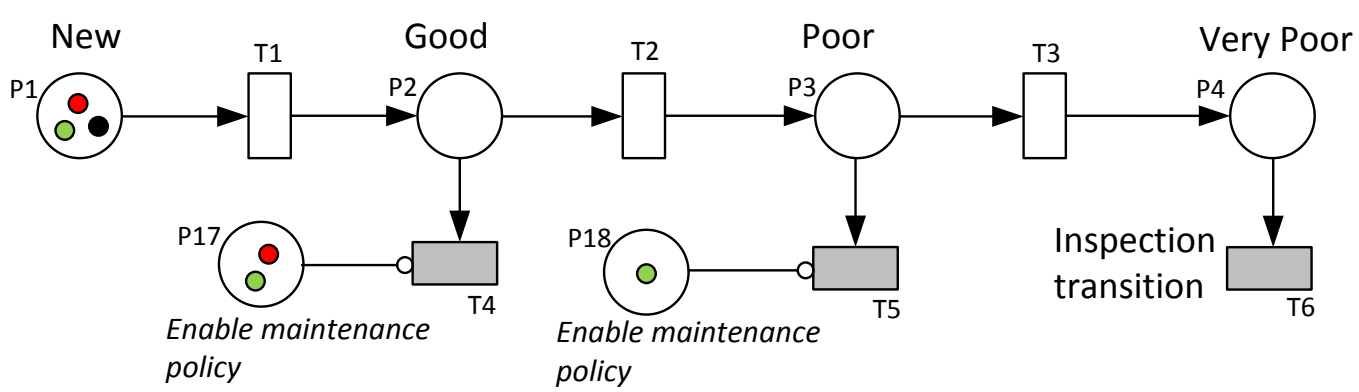

Figure 5.19: Applying intervention option to individual bridge element in the Petrinet bridge model

As can be seen, the PN model allows different intervention options to be applied to each individual bridge component, this increases significantly the possible intervention strategies. Note that, the model is capable of modelling a scenario when intervention options are ignored and the asset is allowed to deteriorate, however this is not a practical option. Therefore, it was assumed that all components will be replaced as soon as they reach a very poor condition. Additionally, by not considering this option, the search space reduces in the optimisation problem.

For the modelled bridge with 8 major elements used in this project, all possible maintenance strategies can be represented by the matrix below (Figure 5.20) where each row represents a particular intervention scenario, each matrix element shows the presence of a token in places $\mathrm{P} 17$ or $\mathrm{P} 18$. The first eight columns indicate the marking of tokens in place $\mathrm{P} 17$ and the second eight columns indicate the marking of tokens in place P18. In particular, the first row of the matrix, where all the matrix elements are zeros, indicates that there are no tokens in places $\mathrm{P} 17$ and P18. Thus the first row represents the intervention option which all components are repaired as soon as they are in the state where repair is possible. The second row indicates that token number 1 is marked in place P17, this means that, only for 
the bridge element modelled by token 1 , minor repair is disabled, i.e. the component is allowed to deteriorate to a poor condition. Similarly, the last row represents the option which all components are allowed to deteriorate to very poor states. For 8 tokens, there are a total of $2^{(8 \times 2)}=65,536$ possible intervention options.

$$
\left[\begin{array}{cccccccccccccccc}
0 & 0 & 0 & 0 & 0 & 0 & 0 & 0 & 0 & 0 & 0 & 0 & 0 & 0 & 0 & 0 \\
t k_{1} & 0 & 0 & 0 & 0 & 0 & 0 & 0 & 0 & 0 & 0 & 0 & 0 & 0 & 0 & 0 \\
0 & t k_{2} & 0 & 0 & 0 & 0 & 0 & 0 & 0 & 0 & 0 & 0 & 0 & 0 & 0 & 0 \\
\vdots & \vdots & \vdots & \vdots & \vdots & \vdots & \vdots & \vdots & \vdots & \vdots & \vdots & \vdots & \vdots & \vdots & \vdots & \vdots \\
t k_{1} & t k_{2} & t k_{3} & t k_{4} & t k_{5} & t k_{6} & t k_{7} & t k_{8} & t k_{1} & t k_{2} & t k_{3} & t k_{4} & t k_{5} & t k_{6} & t k_{7} & t k_{8}
\end{array}\right]
$$

Figure 5.20: All possible maintenance strategies for a bridge contains 6 elements

\subsubsection{Complete bridge model}

Using the same net for all bridge elements, a complete bridge can be modelled by adding more tokens to the net as demonstrated in Figure 5.21. Each component is represented by a single token in the element net. If the component is a metal element with a protective coating, a linked token is added to the coating net.

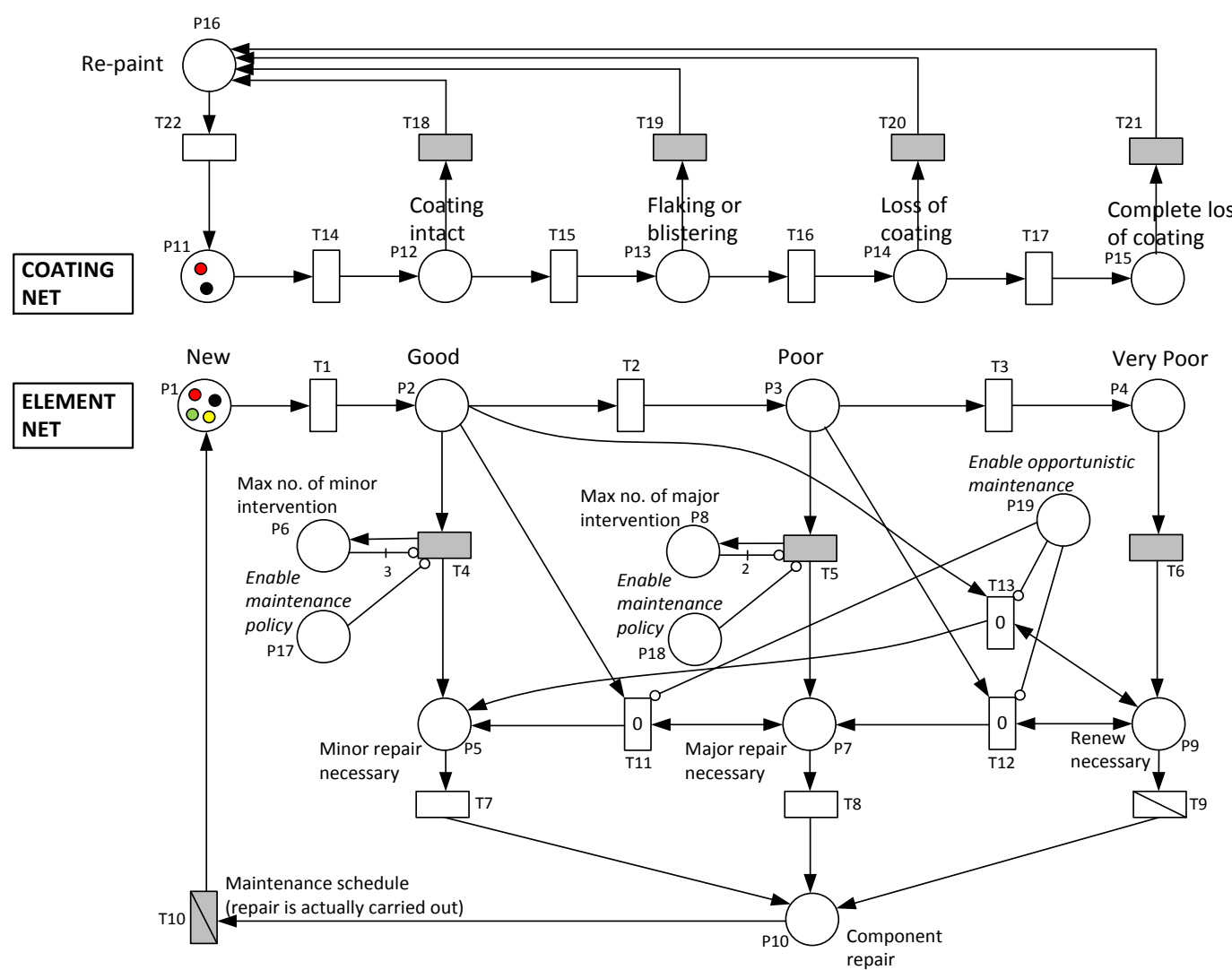

Figure 5.21: Modelling multi-components by adding more tokens

\subsubsection{Model assumptions}

- The repair of any metal element also restores the condition of the coating i.e. new layer of paint is applied after repair. 
- Inspections are assumed to be perfect, that is, the real element deterioration state can be detected without error.

- Maintenance is also modelled as perfect that is a repair will restore the system to the 'as good as new' condition.

\subsection{Model analysis}

\subsubsection{Model construction and simulation}

So far, a PN model has been formulated to model the dynamic process of the change in states of a bridge asset. The net changes state when an event occurs and for many stochastic process modelled in the net, these changes occur at random time. Simulation is required to simulate all of the changes in the system. Therefore, the developed PN bridge model provides a framework for simulation where many processes and rules are incorporated.

There are several commercially available programs that can simulate the PN model, however, due to the modifications to the original PN modelling technique, a computer program was written to accommodate the generation and solution of the PN bridge model. The model is used to simulate the bridge and its elements' conditions and the effects of maintenance over a 60 year lifetime period.

\subsubsection{Monte Carlo sampling}

Sampling from distributions is required for all stochastic transitions. For all the transitions that are governed by a Weibull distribution, Weibull $(\eta, \beta)$, the transition times are derived by generating a random probability and the sampled transition time is calculated using the equation below:

where

$$
t=\eta[-(\ln (X))]^{\frac{1}{\beta}}
$$

$t$ is the sample time

$X$ is a random number between 0 and 1

The random sampling process is repeated for a number of times (number of simulations) and statistics are collected. These statistics indicate the performance and characteristics of the system. The PN bridge model allows many types of characteristics 
to be collected whether it is on a single modelled component or the whole bridge system. These statistics are presented and discussed in the next section.

\subsubsection{Convergence analysis}

With random sampling, the confidence of the model results increases with the number of simulations. Running more simulations gives more precise results, but it is also timeconsuming. Convergence occurs when running further simulations does not change the model results significantly. By setting a threshold level, convergence is achieved by running fewer simulations. The analysis in this project is considered to converge when the changes in the model results are less than $1 \%$. It was found that this is achieved by running more than 200 simulations.

\subsection{Model application}

\subsubsection{Asset selection}

The asset selected is the same as the one chosen in the previous chapter (Aston Hall Bridge - Chapter 4 - 4.3.1). The asset represents a typical metal underbridge in the population and enables results to be compared with those of the Markov model. The bridge main elements and their initial conditions are described again in the table below:

\begin{tabular}{|l|c|c|}
\hline \multicolumn{1}{|c|}{ Component } & Material & Initial condition \\
\hline Deck (DCK) & Concrete & Good \\
\hline External main girder 1 (MGE 1) & Metal & Good \\
\hline Internal main girder (MGI) & Metal & Poor \\
\hline External main girder 2 (MGE 2) & Metal & Good \\
\hline Bearing 1 (BGL 1) & Metal & Poor \\
\hline Bearing 2 (BGL 2) & Metal & Poor \\
\hline Abutment (ABT 1) & Masonry & As New \\
\hline Abutment (ABT 1) & Masonry & As New \\
\hline
\end{tabular}

Table 5.1: Asset major components and initial conditions

\subsubsection{Model inputs and parameters}

The Petri-Net bridge model for the selected asset is illustrated in Figure 5.22. The model parameters are given in Table 5.2 to Table 5.5. Transitions $\mathrm{T} 1, \mathrm{~T} 2$ and $\mathrm{T} 3$ are conditional transitions which mean that the transition times are sampled from different distributions depending on the token type fired through the transition. Table 5.2 shows the different distributions for different 
bridge components and their respective materials. Note that the distributions of the transition times represented by $\mathrm{T} 2$ and $\mathrm{T} 3$ are convolution transitions estimated using simulations as discussed in section 5.2.5.

For metal elements, where the coating is also modelled, the transition times generated by $\mathrm{T} 1, \mathrm{~T} 2$ and $\mathrm{T} 3$ are also sampled from different distributions as given in Table 5.3. It is assumed that the transition rates of metal elements with the new coating are the same as the degradation rates obtained in Table 5.2. It was also assumed that for each worse coating condition, the degradation increases by $5 \%$ i.e. the eta value decreases by $5 \%$. The transition rates between each coating condition are given in Table 5.4 .

Constant transition times are given in Table 5.5. For periodical transition T4-6 and T18-21, the transition can only fire at certain times. This was set to every 6 years, which is also the same as the interval between inspections. Similarly, transition T10 was set to be enabled after every 1 year, this is to model the maintenance schedule of one time a year for any maintenance. The transition times for transitions T7-9 are 1, 2 and 3 years, these times represent the delay in each type of maintenance (minor, major repair and renewal). The transition times for transition T22 is the time it takes to restore the coating condition back to new and was assumed to be one week.

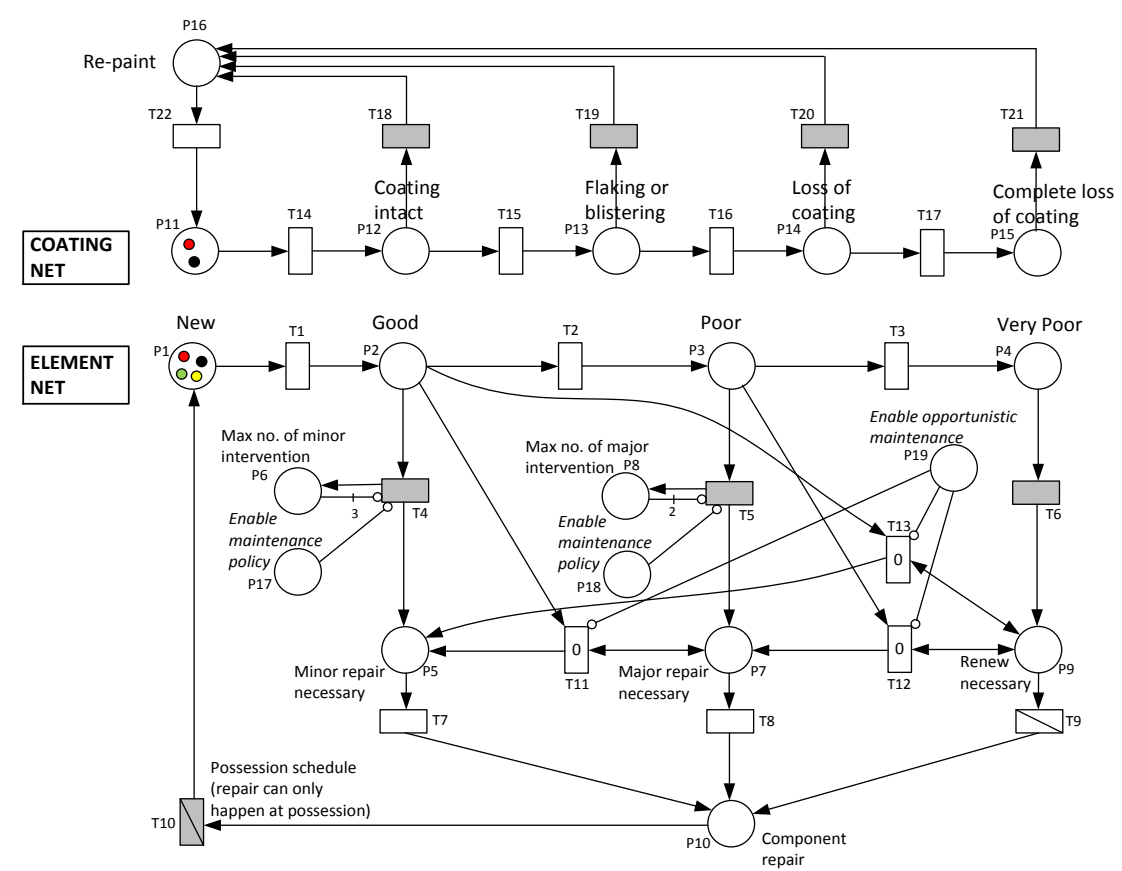

Figure 5.22: Petri-Net bridge model 


\begin{tabular}{|c|c|c|c|c|c|c|c|c|c|c|c|c|}
\hline \multirow{4}{*}{$\begin{array}{c}\text { Component } \\
\text { Material } \\
\text { Transition ID } \\
\end{array}$} & \multicolumn{12}{|c|}{ Stochastic transition time (years) } \\
\hline & \multirow{2}{*}{\multicolumn{2}{|c|}{$\begin{array}{l}\text { Girder } \\
\text { Metal }\end{array}$}} & \multicolumn{6}{|c|}{ Decking } & \multirow{2}{*}{\multicolumn{2}{|c|}{$\begin{array}{c}\text { Bearing } \\
\text { Metal }\end{array}$}} & \multirow{2}{*}{\multicolumn{2}{|c|}{$\begin{array}{c}\text { Abutment } \\
\text { Masonry }\end{array}$}} \\
\hline & & & \multicolumn{2}{|c|}{ Metal } & \multicolumn{2}{|c|}{ Concrete } & \multicolumn{2}{|c|}{ Timber } & & & & \\
\hline & Beta & Eta & Beta & Eta & Beta & Eta & Beta & Eta & Beta & Eta & Beta & Eta \\
\hline $\mathrm{T} 1$ & 1.71 & 23.39 & 1.27 & 10.28 & 1.08 & 19.09 & 1.31 & 3.99 & 0.84 & 14.94 & 1.00 & 51.94 \\
\hline $\mathrm{T} 2$ & 3.42 & 27.10 & 3.63 & 10.10 & 2.95 & 11.00 & 2.35 & 5.00 & 3.27 & 5.00 & 3.47 & 53.00 \\
\hline T3 & 3.78 & 114.20 & 2.81 & 12.70 & 2.49 & 14.30 & 1.90 & 5.00 & 3.53 & 9.50 & 3.25 & 71.6 \\
\hline
\end{tabular}

Table 5.2: Transition rates for Transition T1, T2 and T3 depending on the type of token (component type and component material)

\begin{tabular}{|c|c|c|c|c|c|c|c|c|c|c|}
\hline Metal Element & \multicolumn{9}{|c|}{ Stochastic transition time (years) } \\
\hline $\begin{array}{c}\text { Coating } \\
\text { condition }\end{array}$ & \multicolumn{1}{|c|}{ New Coating } & \multicolumn{2}{|c|}{ Coating intact } & \multicolumn{2}{|c|}{$\begin{array}{l}\text { Flaking or } \\
\text { blistering }\end{array}$} & Loss of coating & \multicolumn{2}{c|}{$\begin{array}{c}\text { Complete loss } \\
\text { of coating }\end{array}$} \\
\hline Transition ID & Beta & Eta & Beta & Eta & Beta & Eta & Beta & Eta & Beta & Eta \\
\hline T1 & 1.71 & 23.39 & 1.71 & 22.2 & 1.71 & 21.05 & 1.71 & 19.88 & 1.71 & 17.78 \\
\hline T2 & 3.42 & 27.10 & 3.42 & 25.7 & 3.42 & 24.39 & 3.42 & 23.04 & 3.42 & 20.60 \\
\hline T3 & 3.78 & 114.20 & 3.78 & 108 & 3.78 & 102.78 & 3.78 & 97.07 & 3.78 & 86.79 \\
\hline
\end{tabular}

Table 5.3: Transition rates for Transition T1, T2 and T3 for Metal element depending on the condition of the coating

\begin{tabular}{|c|c|c|}
\hline Metal Coating & \multicolumn{2}{|c|}{ Stochastic transition time (years) } \\
\hline Transition ID & Beta & Eta \\
\hline T14 & 1.0 & 5 \\
\hline T15 & 1.0 & 5 \\
\hline T16 & 1.0 & 5 \\
\hline T17 & 1.0 & 5 \\
\hline
\end{tabular}

Table 5.4: Transition rates for the coating of metal element (Transition T11 - T14)

\begin{tabular}{|c|c|c|c|c|c|c|c|c|}
\hline Transition ID & T4-6 & T7 & T8 & T9 & T11-13 & T10 & T18-21 & T22 \\
\hline Fix transition time (years) & 6 & 1 & 2 & 3 & 0 & 1 & 6 & 0.08 \\
\hline
\end{tabular}

Table 5.5: Fixed transition times for periodical transition (T4-6, T10, and T15-T18) and transition (T7-9, T18-21, and T22)

\subsubsection{Element analysis}

To model the selected bridge structure, 8 tokens were added to the PN bridge model (Figure 5.22), each token represented the major bridge elements modelled. Thus, statistics obtained for any one token would give the predicted performance of a bridge element.

Figure 5.23 shows an example of the bridge deck life over a simulated life of 500 years. Note that the 500 years prediction period is chosen only for the illustration purpose as this is unrealistic prediction. The graph demonstrates a simulated life of the bridge deck in terms of the time it resides in a condition state before moving to a worse condition (degradation process) or moving to the 'as good as new' condition (repair process). Over the simulated life time, the time that the token resides in each 
place in the model can be tracked. Carrying out this simulation for a number of times, statistics are then collected to provide a performance indication of each bridge element.

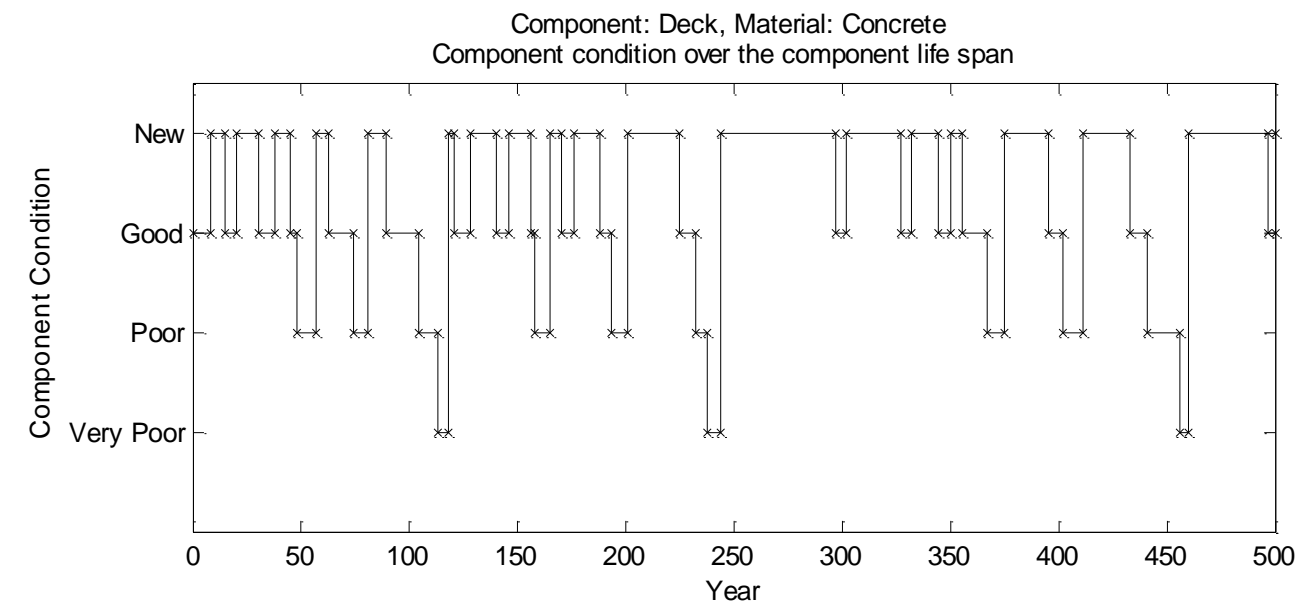

Figure 5.23: Example of one simulated life time of a bridge deck residing in different condition states

Figure 5.24 shows the mean time of the bridge deck residing in the 'as new' state is expected to be around 40 years over the 60 years simulation time. For 200 simulations carried out, it can be seen that, for the component, the convergence was achieved at around 120 simulations.

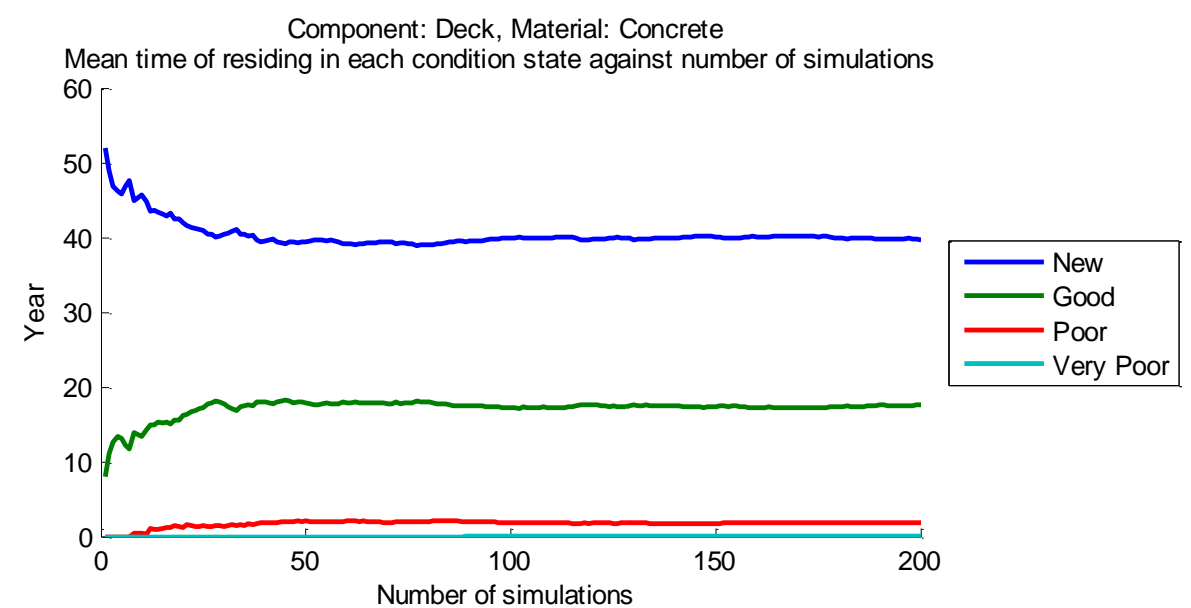

Figure 5.24: Duration of staying in each condition states against the number of simulation - bridge deck - maintenance strategy: repair as soon as possible

The probabilities of being in each condition state of the bridge deck are shown in Figure 5.25. Since the probability of being in a particular condition directly relates to the time the component spends in that state, the plot profile seen in Figure 5.25 is similar to Figure 5.24. It is expected that, following this maintenance strategy (strategy 1 - repair as soon as possible for all components), there is a $67 \%$ probability that the bridge deck will 
be in the 'as new' condition, just above $30 \%$ probability of it being in a good condition and very little probability of it being in a poor or a very poor condition.

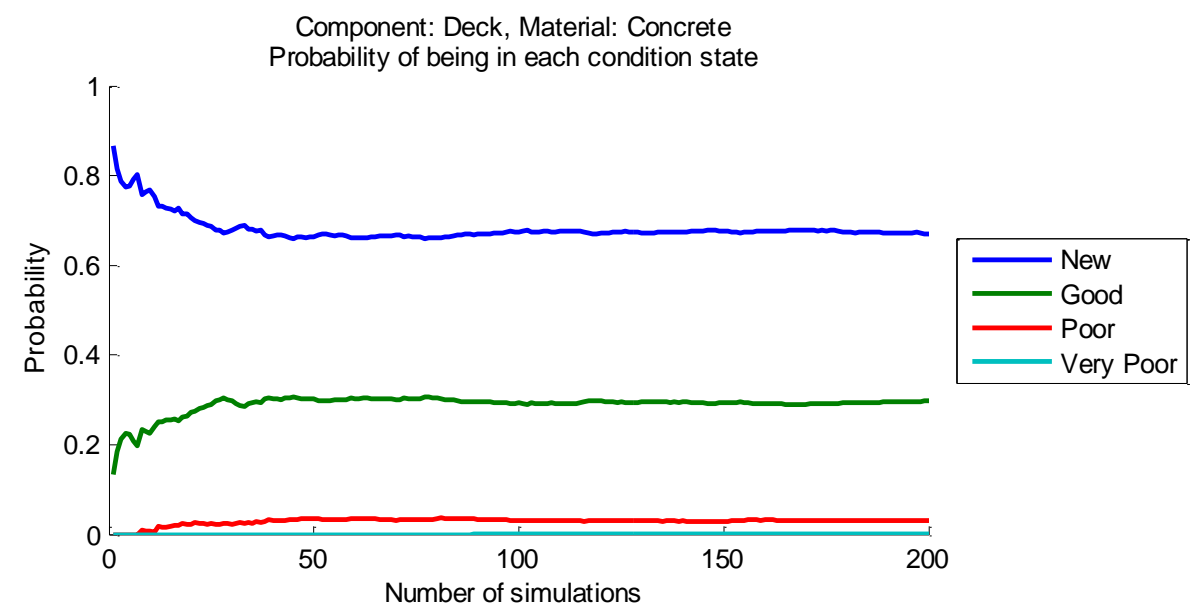

Figure 5.25: Probability of being each condition state for the bridge deck maintenance strategy: repair as soon as possible

Figure 5.26 shows that we expect minor repairs to be carried out two or three times over the life time of the component. It is predicted that there is no deck replacement, this agrees with the fact that the expected probabilty of the deck being in a very poor condition is almost zero. With the information about the unit cost for each type of repair, the expected maintenance costs can easily be deduced.

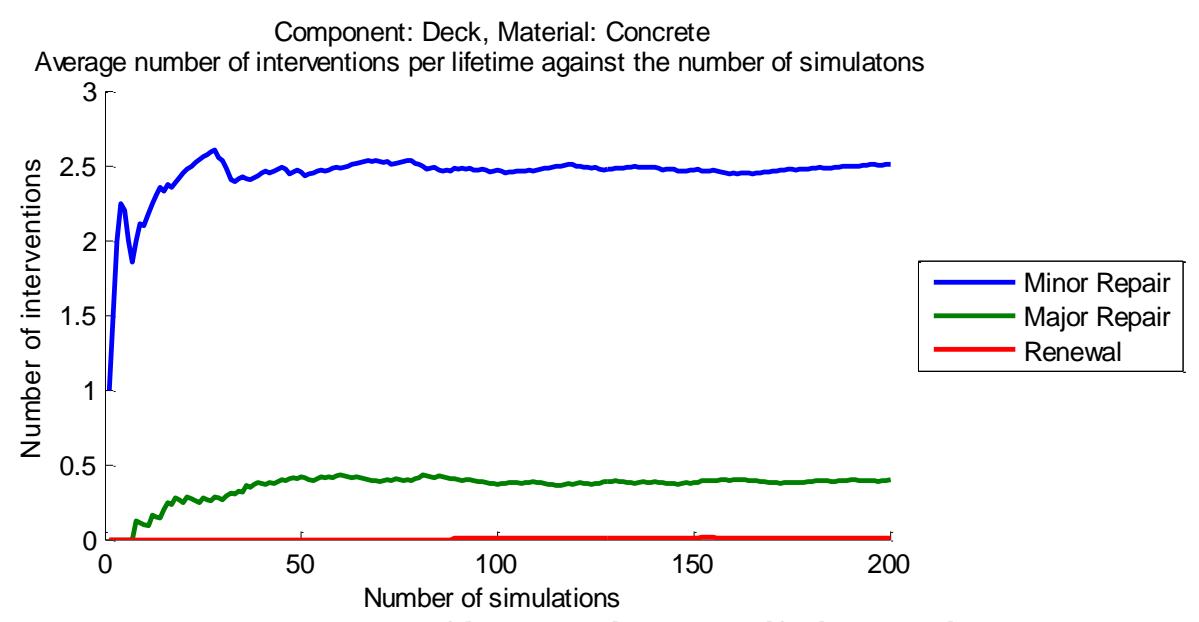

Figure 5.26: Average number of interventions per lifetime against the number of the simulations - bridge deck - maintenance strategy: repair as soon as possible

The average condition disitribution at the end of each year can be seen in Figure 5.27. Given the initial condition of the bridge deck is in a good condition, the probability of the bridge deck being in this condition is 1 at year 0 (the start of the simulation). In the following years, this probability decreases because the deck starts 
to deteriorate, along with it, the probability of the deck being in the poor condition increases. After 6 years, when the first inspection is carried out, the component condition is revealed. Depending on the condition of the bridge deck, an appropriate repair is scheduled and the effect of maintenance can be seen in the increasing probability of the bridge deck being in the 'as new' condition. Note that the effect does not happen immediately after 6 years because there is a delay time ( 1 to 3 years depending on the type of repair) associated with the repair process. Therefore the increasing in the probabilty of being in the 'as new' state can be seen happening around the $7^{\text {th }}$ to $9^{\text {th }}$ year. Carrying on further into the predicted life time, the deterioration process as well as inspection and maintenance process is reflected in the wave nature of the plot.

This section presented only the analysis on the bridge deck, for other bridge components, the results are shown in Appendix D-2.

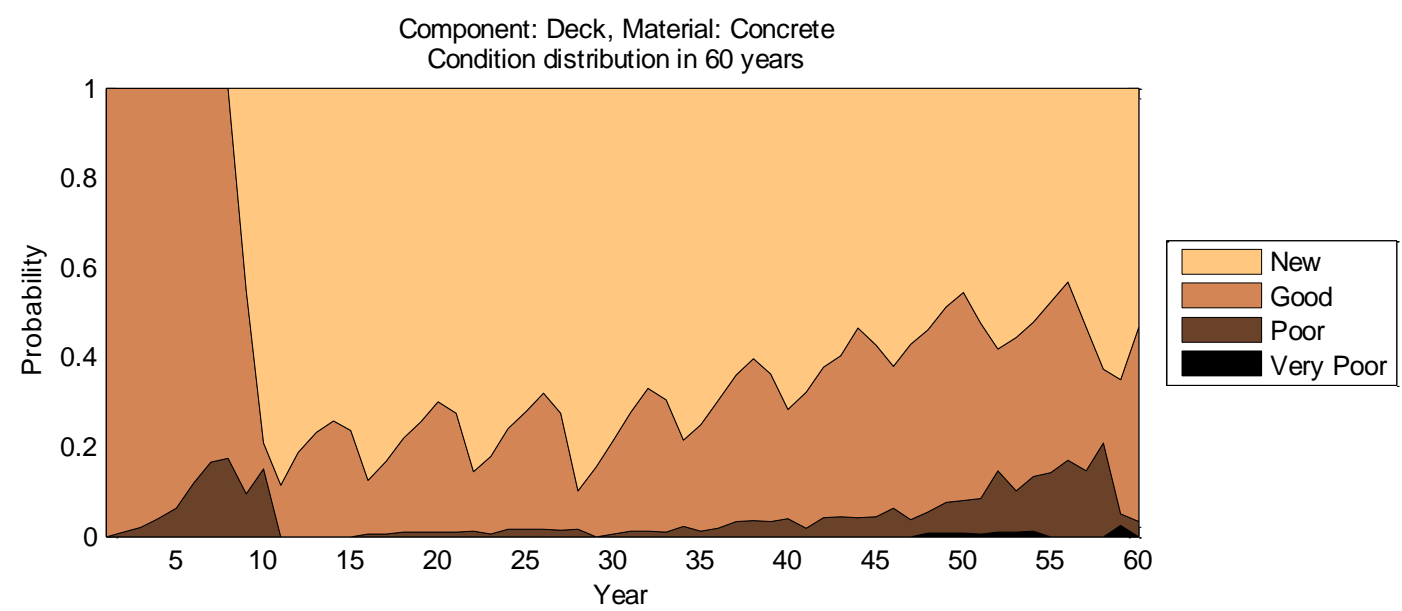

Figure 5.27: Condition distribution at the end of each year for the bridge deck maintenance strategy: repair as soon as possible

\subsubsection{System analysis}

By carrying out analysis on all elements of the bridge, the performance of the whole bridge system can be seen. Table 5.6 and Table 5.7 show the summary of the system statistics obtained for all bridge elements when applying maintenance strategy of intervening as soon as any degraded state is discovered. It can be seen that, it is predicted that at least one minor intervention is necessary on all components over their lifetimes. Also, with this maintenance strategy, the average time that the bridge is in the 'as new' condition is roughly around 40 years over the 60 years prediction period. This detailed 
information allows the investigation of the effects of different specified maintenance strategies in terms of performance and cost.

\begin{tabular}{|c|c|c|c|c|c|c|c|c|c|}
\hline & & DCK & MGE1 & MGI & MGE2 & BGL1 & BGL2 & ABT1 & ABT2 \\
\hline \multirow{4}{*}{ Minor intervention } & Minimum number achieved & 0 & 0 & 0 & 1 & 0 & 0 & 0 & 0 \\
\cline { 2 - 10 } & Maximum number achieved & 4 & 3 & 3 & 3 & 3 & 3 & 3 & 3 \\
\cline { 2 - 10 } & Average & 2.51 & 2.62 & 1.80 & 2.61 & 1.70 & 1.61 & 1.05 & 1.04 \\
\cline { 2 - 10 } & Standard deviation & 0.74 & 0.57 & 0.75 & 0.57 & 1.03 & 1.01 & 0.82 & 0.88 \\
\hline \multirow{5}{*}{ Major intervention } & Minimum number achieved & 0 & 0 & 1 & 0 & 0 & 0 & 0 & 0 \\
\cline { 2 - 10 } & Maximum number achieved & 2 & 1 & 1 & 1 & 2 & 2 & 0 & 0 \\
\cline { 2 - 10 } & Average & 0.40 & 0.03 & 1 & 0.03 & 1.45 & 1.42 & 0 & 0 \\
\cline { 2 - 10 } & Standard deviation & 0.54 & 0.16 & 0 & 0.16 & 0.62 & 0.60 & 0 & 0 \\
\hline \multirow{4}{*}{ Replacement } & Minimum number achieved & 0 & 0 & 0 & 0 & 0 & 0 & 0 & 0 \\
\cline { 2 - 10 } & Maximum number achieved & 1 & 0 & 0 & 0 & 2 & 1 & 0 & 0 \\
\cline { 2 - 10 } & Average & 0.01 & 0 & 0 & 0 & 0.27 & 0.27 & 0 & 0 \\
\cline { 2 - 10 } & Standard deviation & 0.10 & 0 & 0 & 0 & 0.46 & 0.45 & 0 & 0 \\
\hline
\end{tabular}

Table 5.6: Statistics on the expected number of interventions on each bridge components - maintenance strategy: repair as soon as possible

\begin{tabular}{|c|c|c|c|c|c|c|c|c|c|}
\hline & & DCK & MGE1 & MGI & MGE2 & BGL1 & BGL2 & ABT 1 & ABT 2 \\
\hline \multirow{4}{*}{ As new condition } & $\begin{array}{c}\text { Minimum number } \\
\text { achieved }\end{array}$ & 14.53 & 17.41 & 26.85 & 20.13 & 13.87 & 10.48 & 41.53 & 31.21 \\
\hline & $\begin{array}{c}\text { Maximum number } \\
\text { achieved }\end{array}$ & 52.00 & 52.00 & 51.00 & 52.00 & 51.00 & 51.00 & 60.00 & 60.00 \\
\hline & Average & 39.70 & 42.07 & 41.87 & 42.29 & 37.34 & 37.41 & 54.38 & 54.38 \\
\hline & Standard deviation & 8.14 & 5.98 & 4.60 & 6.12 & 7.72 & 8.27 & 4.57 & 4.91 \\
\hline \multirow{4}{*}{ Good condition } & $\begin{array}{c}\text { Minimum number } \\
\text { achieved }\end{array}$ & 4.50 & 5.69 & 0 & 8 & 0 & 0 & 0 & 0 \\
\hline & $\begin{array}{c}\text { Maximum number } \\
\text { achieved }\end{array}$ & 35.82 & 41.89 & 21.05 & 38.50 & 21.84 & 21.08 & 18.36 & 28.79 \\
\hline & Average & 17.65 & 17.14 & 8.41 & 16.93 & 9.26 & 9.02 & 4.93 & 4.93 \\
\hline & Standard deviation & 6.80 & 5.62 & 4.06 & 5.92 & 5.18 & 5.36 & 4.29 & 4.71 \\
\hline \multirow{4}{*}{ Poor condition } & $\begin{array}{c}\text { Minimum number } \\
\text { achieved }\end{array}$ & 0 & 0 & 9 & 0 & 2.27 & 2.72 & 0 & 0 \\
\hline & $\begin{array}{c}\text { Maximum number } \\
\text { achieved }\end{array}$ & 19.93 & 5.42 & 9.00 & 4.91 & 25.66 & 23.76 & 0 & 0 \\
\hline & Average & 1.89 & 0.09 & 9.00 & 0.08 & 11.47 & 11.45 & 0 & 0 \\
\hline & Standard deviation & 2.82 & 0.62 & 0.00 & 0.55 & 3.93 & 3.90 & 0 & 0 \\
\hline \multirow{4}{*}{$\begin{array}{l}\text { Very poor } \\
\text { condition }\end{array}$} & $\begin{array}{c}\text { Minimum number } \\
\text { achieved }\end{array}$ & 0 & 0 & 0 & 0 & 0 & 0 & 0 & 0 \\
\hline & $\begin{array}{c}\text { Maximum number } \\
\text { achieved }\end{array}$ & 5.37 & 0 & 0 & 0 & 11.39 & 9.91 & 0 & 0 \\
\hline & Average & 0.04 & 0 & 0 & 0 & 1.23 & 1.41 & 0 & 0 \\
\hline & Standard deviation & 0.40 & 0 & 0 & 0 & 2.34 & 2.47 & 0 & 0 \\
\hline
\end{tabular}

Table 5.7: Statistics on the duration (years) spending in each condition state of each bridge components - maintenance strategy: repair as soon as possible 


\subsubsection{Effects of varying intervention strategies}

The PN model allows the intervention option to be selected for each bridge element individually. There are four intervention options possible for a single element and are given in the Table 5.8 .

\begin{tabular}{|c|c|c|}
\hline Option & Strategy & PN model representation \\
\hline 1 & $\begin{array}{l}\text { Repair as soon as the component is } \\
\text { identified to be in a state where a } \\
\text { repair is required. }\end{array}$ & Place P11 and P12 are un-marked. \\
\hline 2 & $\begin{array}{l}\text { Minor repair is inhibited, only major } \\
\text { repair and replacement is considered. }\end{array}$ & $\begin{array}{l}\text { Place P11 is marked with a token } \\
\text { corresponding to the bridge } \\
\text { component which this strategy is } \\
\text { applied to. }\end{array}$ \\
\hline 3 & $\begin{array}{l}\text { Major repair is inhibited, only minor } \\
\text { repair and replacement is considered. }\end{array}$ & $\begin{array}{l}\text { Place P12 is marked with a token } \\
\text { corresponding to the bridge } \\
\text { component which this strategy is } \\
\text { applied to. }\end{array}$ \\
\hline 4 & $\begin{array}{l}\text { Minor and major repair are inhibited, } \\
\text { only replacement is considered. }\end{array}$ & $\begin{array}{l}\text { Place P11 and P12 are marked with a } \\
\text { token corresponding to the bridge } \\
\text { component which this strategy is } \\
\text { applied to. }\end{array}$ \\
\hline
\end{tabular}

Table 5.8: Four intervention strategies possible for a single bridge component

The effect of these strategies on the bridge external main girder 1 (MGE 1) is illustrated from Figure 5.28 to Figure 5.31. With intervention option 1 and 3, where minor repair is enabled, very similar condition profiles are shown. However since the third option inhibits major repairs, the girder is allowed to stay in the poor condition without any intervention. This is reflected in the rise in the probability of being in a poor condition toward the end of the simulation period, hence the probability of being in the 'as new' condition decreases.

In option 2, the component can deteriorate past the good condition and an intervention is only carried out when the condition is revealed to be in a poor or very poor condition. Figure 5.29 shows that the probability of the girder being in a poor condition increases in the first 30 years before gradually decreases in the following 15 - 20 years. This means at least one major repair is predicted to be carried out throughout the predicted component life. This is also demonstrated in Table 5.9, as the average number of predicted major interventions for this option is 1.21 .

Figure 5.31 illustrates that the condition of the bridge main girder is very likely to be in a poor or very poor condition when applying intervention option 4. However with the slow rate of degradation, 
it is predicted that, for this given time period of 60 years, the main girder is unlikely to require a complete replacement (Table 5.9 shows the average number of replacement is less than one) and is more likely to reside in a poor condition.

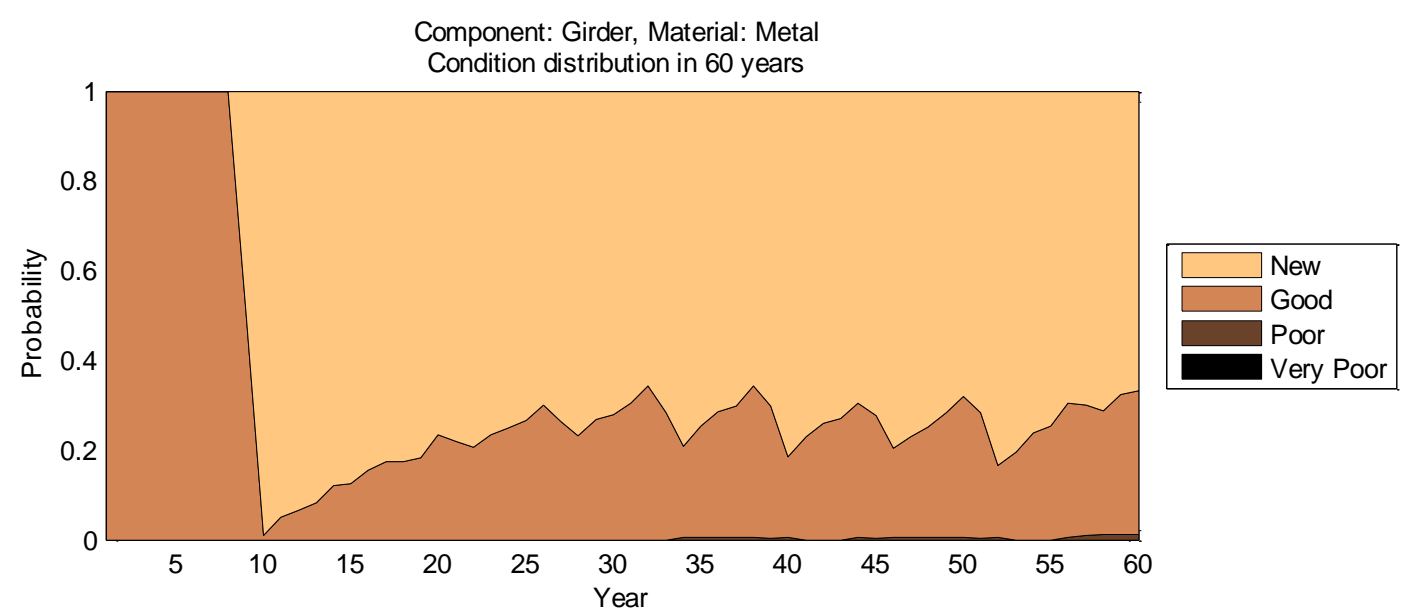

Figure 5.28: Condition distribution at the end of each year for the external main girder (MGE 1) - intervention option 1

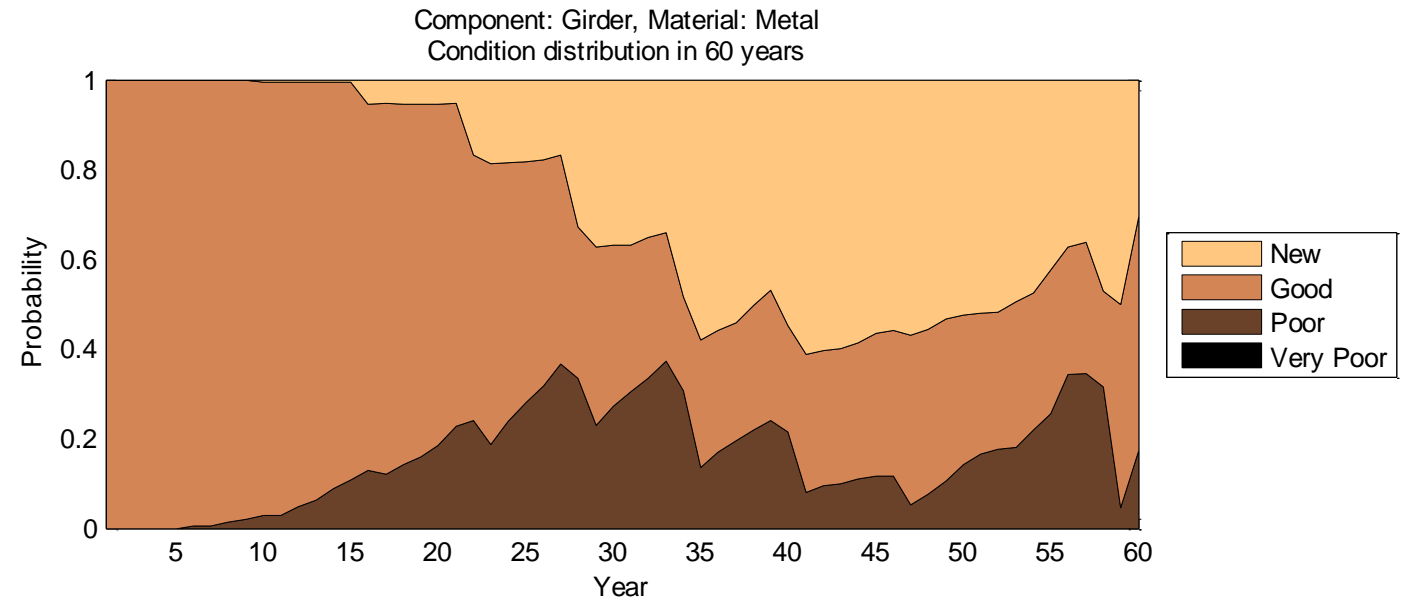

Figure 5.29: Condition distribution at the end of each year for the external main girder (MGE 1) - intervention option 2

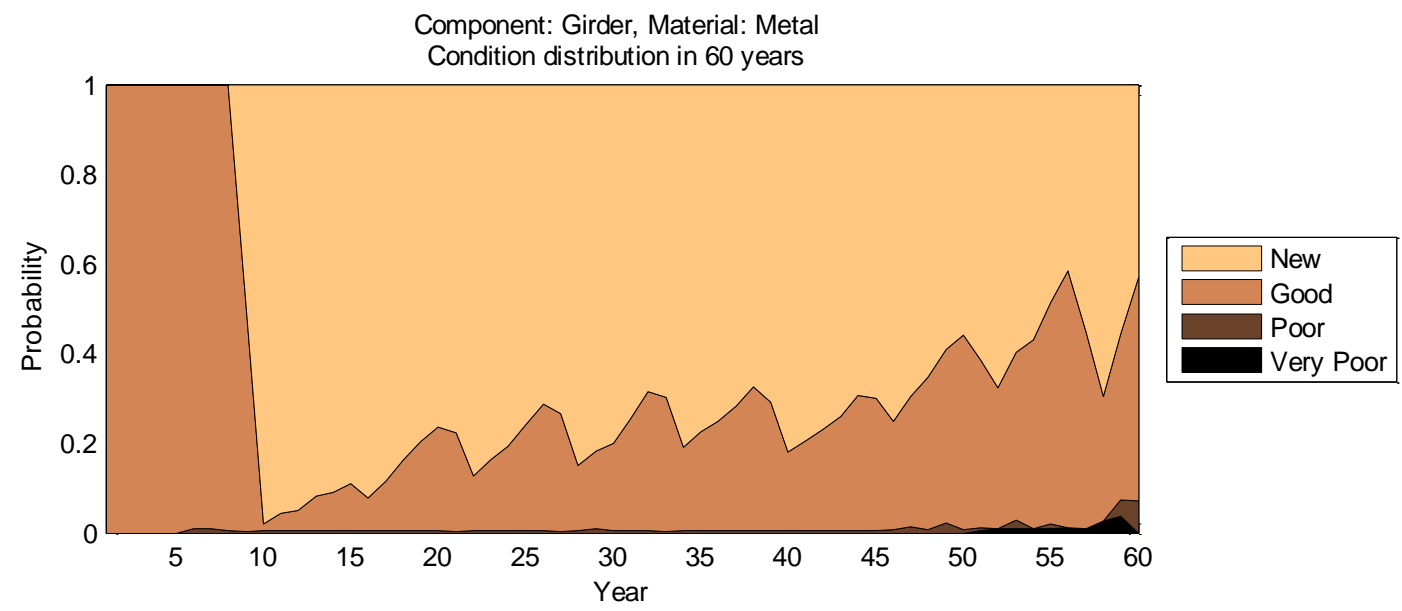

Figure 5.30: Condition distribution at the end of each year for the external main girder (MGE 1) - intervention option 3 


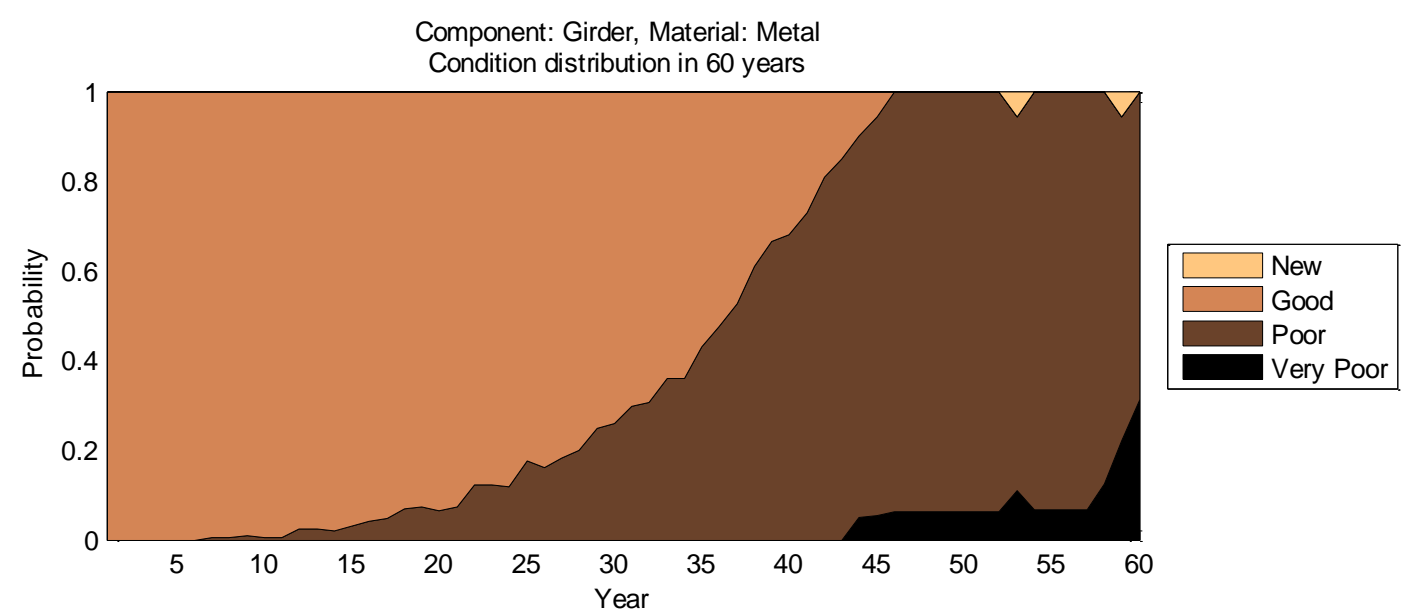

Figure 5.31: Condition distribution at the end of each year for the external main girder (MGE 1) - intervention option 4

\begin{tabular}{|c|c|c|c|c|c|}
\hline & & Option 1 & Option 2 & Option 3 & Option 4 \\
\hline \multirow{4}{*}{ Minor intervention } & Minimum number achieved & 1 & 0 & 0 & 0 \\
\cline { 2 - 6 } & Maximum number achieved & 3 & 0 & 3 & 0 \\
\cline { 2 - 6 } & Average & 2.66 & 0 & 2.625 & 0 \\
\cline { 2 - 6 } & Standard deviation & 0.52 & 0 & 0.597 & 0 \\
\hline \multirow{4}{*}{ Meplacem intervention } & Minimum number achieved & 0 & 1 & 0 & 0 \\
\cline { 2 - 6 } & Maximum number achieved & 1 & 2 & 0 & 0 \\
\cline { 2 - 6 } & Average & 0.02 & 1.21 & 0 & 0 \\
\cline { 2 - 6 } & Standard deviation & 0.14 & 0.41 & 0 & 0 \\
\hline & Minimum number achieved & 0 & 0 & 0 & 0 \\
\cline { 2 - 6 } & Maximum number achieved & 0 & 0 & 1 & 1 \\
\cline { 2 - 6 } & Average & 0 & 0 & 0.005 & 0.065 \\
\cline { 2 - 6 } & Standard deviation & 0 & 0 & 0.071 & 0.247 \\
\hline
\end{tabular}

Table 5.9: Statistics on the expected number of interventions on the external main girder (MGE 1) - all 4 intervention options

It is important to know that the four possible intervention options are for a single bridge component. The PN bridge model has the ability to apply these options individually to each element, for example, intervention option 2 can be applied to the deck, option 3 to the girder, option 1 to the bearing, etc. Therefore, for the whole bridge asset, the maintenance scenarios will increase depending on the number of modelled components. It is not possible to demonstrate the effects of all these scenarios, although statistics were collected for when these 4 intervention options are being applied to all the components. Tables of obtained statistics are presented in Appendix D-3. Figure 5.32 demonstrates the effects of these 4 strategies on the asset average condition. It is obvious for strategies 1,2 and 4 , as the condition that triggers maintenance gets lower at each of the 
strategy, the predicted asset average condition would also be lower progressively. Since strategy 3 considers replacement and also minor repair, the average asset condition is maintained at a higher condition comparing with strategy 4 and is between predicted average condition for strategies 1 and 2 .

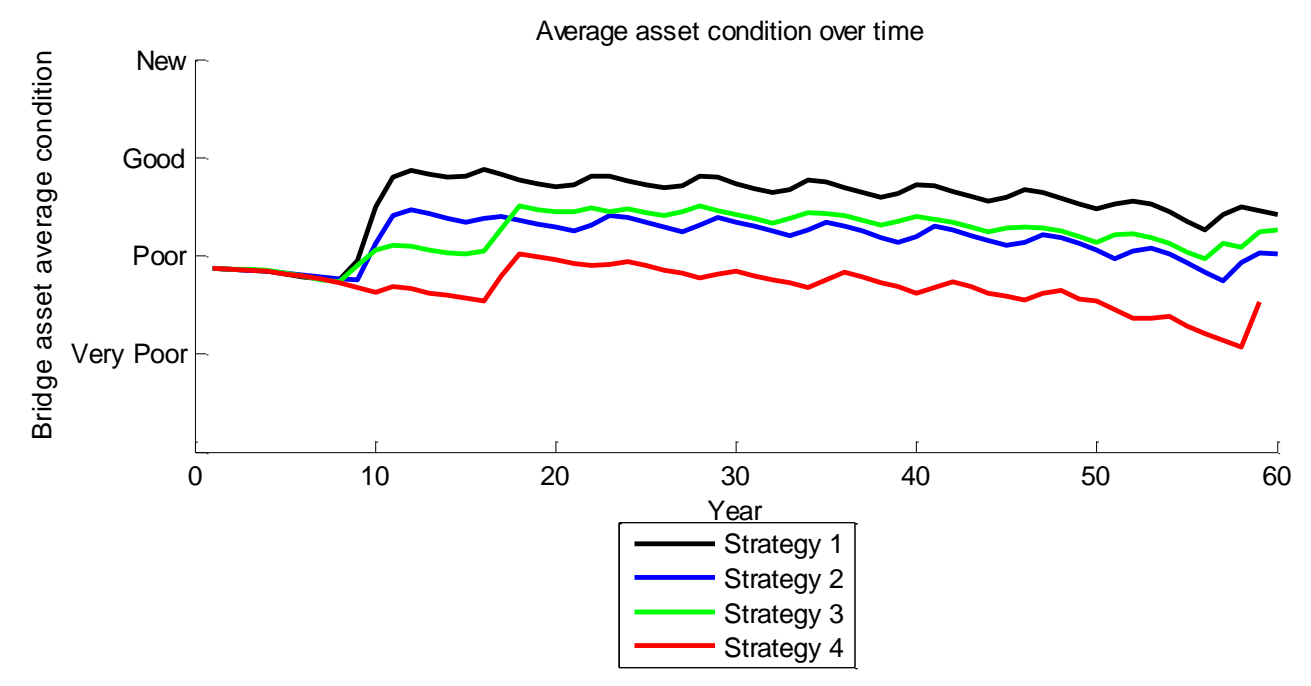

Figure 5.32: Effects of different intervention strategies on the average asset condition

\subsubsection{Effects of opportunistic maintenance}

The effect of opportunistic maintenance is demonstrated in Figure 5.33 and Figure 5.34. The plots show the probabilities of being in each condition state of the external main girder 1 (MGE1) when the maintenance policy 2 is employed. Under this policy the girders are allowed to deteriorate to the poor conditions where major intervention is carried out. For the three main girders being modelled, the internal main girder - MGI is already in the poor condition whilst the other two girders are in good conditions. Thus, as soon as after the first inspection at the $6^{\text {th }}$ year, the MGI will be scheduled for a major repair. This gives an opportunity to carry out opportunistic repair on other main girders (MGE1 and MGE2). Opportunistic maintenance can be seen in Figure 5.34 at around the $7^{\text {th }}$ year, where an opportunistic repair happens and the probability of the MGE1 being in the 'as new' state is almost 1. Figure 5.33 shows that, when opportunistic maintenance is not considered, the MGE1 is allowed to deteriorate to the poor state. This is reflected in a high probability of remaining in the good state and the increasing probability of being in the poor state for a period after the first inspection. Opportunistic maintenance increases the probability of the components being in better 
conditions, thus maintenance policies with opportunistic maintenance is expected to maintain higher asset average conditions. This is observed and confirmed in Figure 5.35.

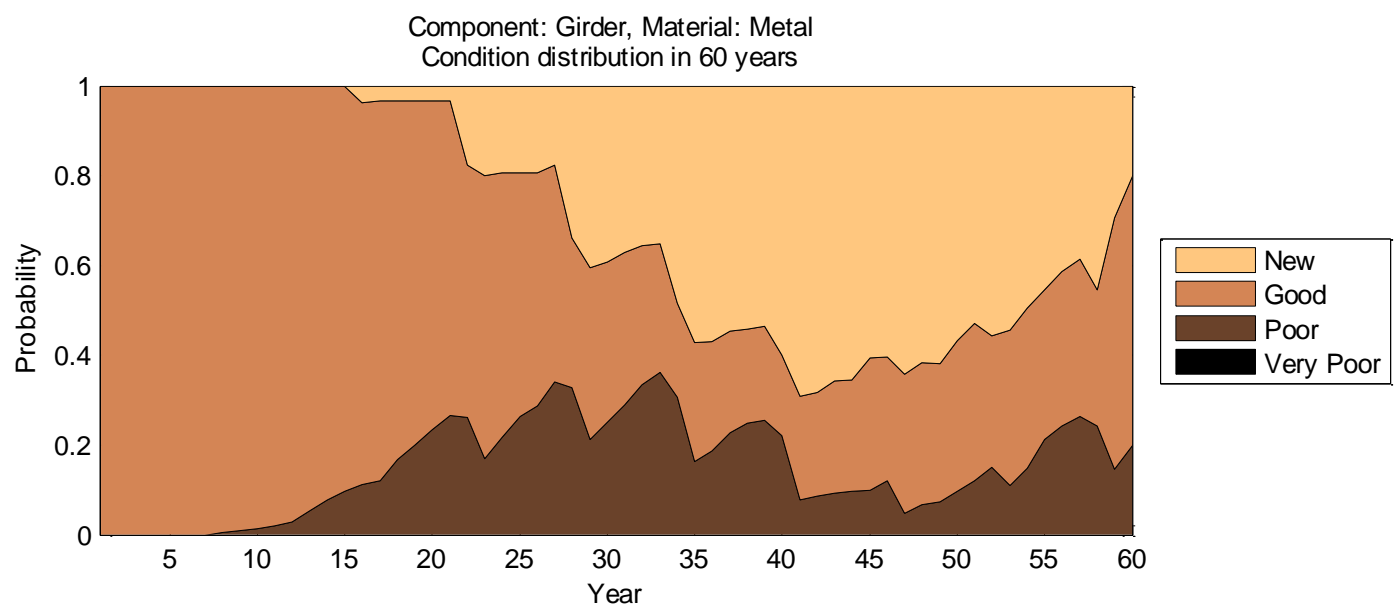

Figure 5.33: Condition distribution at the end of each year for the external main girder (MGE 1) - intervention option 2 without opportunistic maintenance

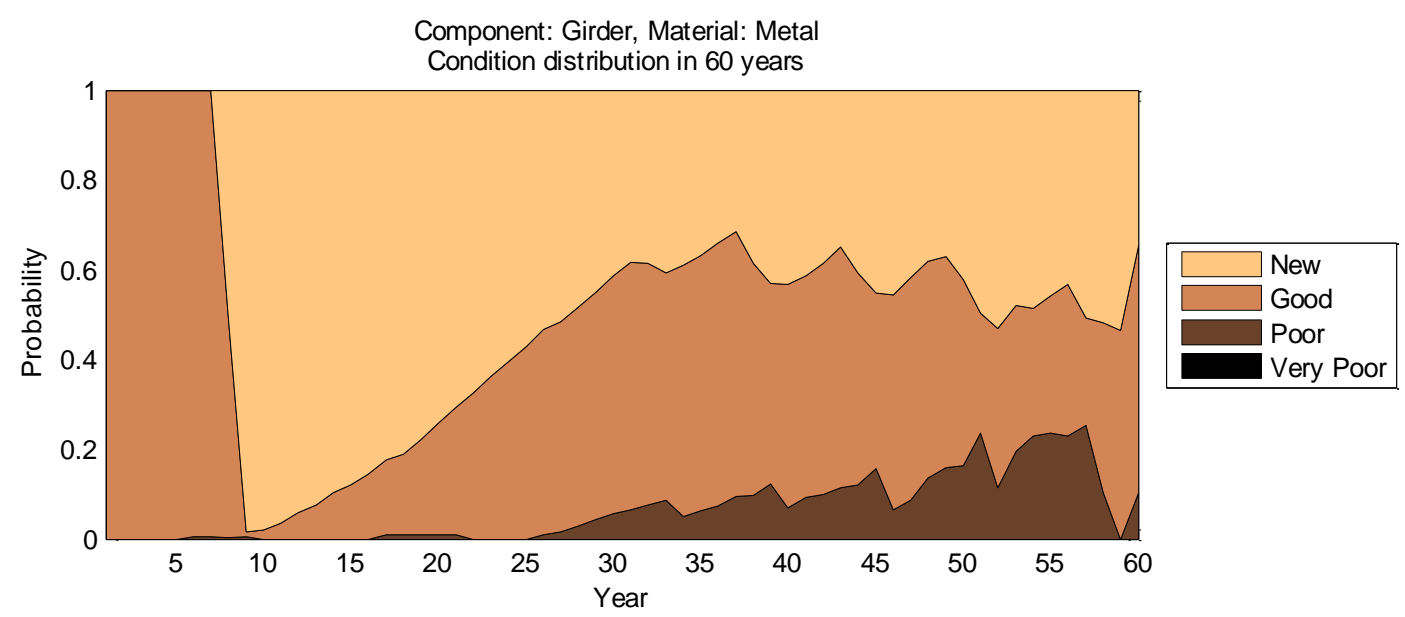

Figure 5.34: Condition distribution at the end of each year for the external main girder (MGE 1) - intervention option 2 with opportunistic maintenance

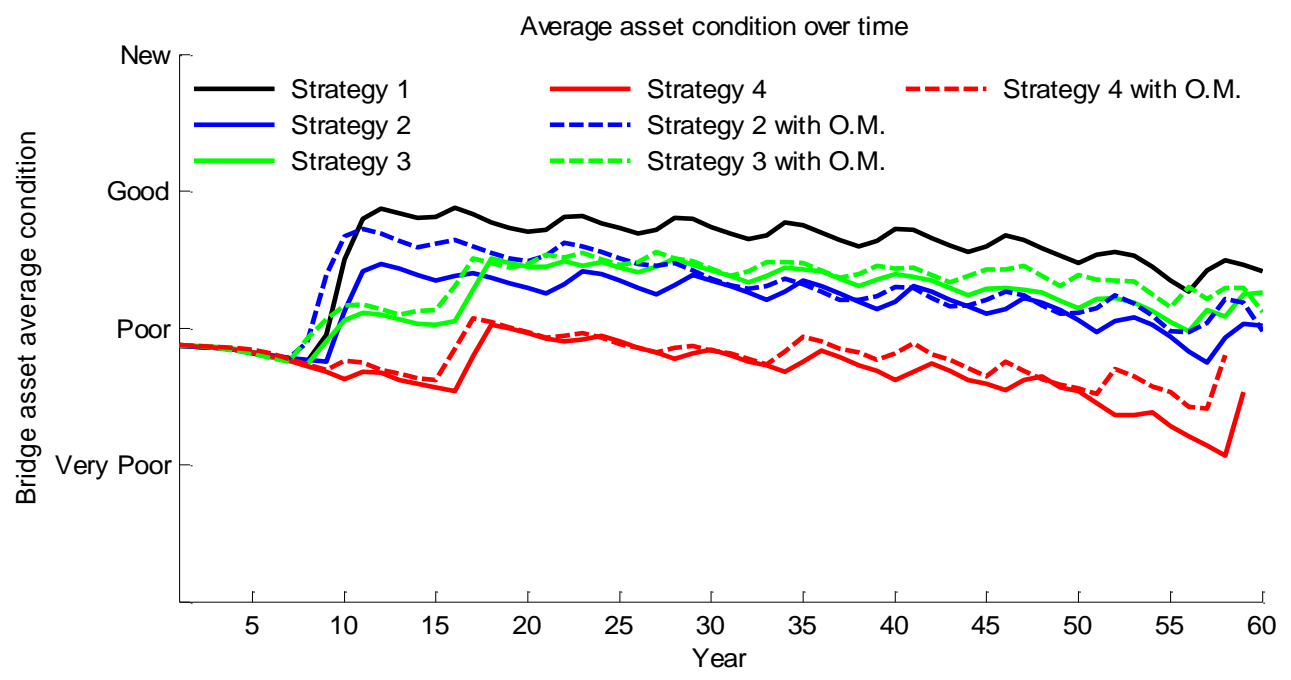

Figure 5.35: Effects of opportunistic maintenance (O.M.) on the average asset condition with different maintenance policies 


\subsubsection{Effects of varying inspection and servicing interval}

Figure 5.36 shows the average probability of the bridge deck being in each condition state over 60 years prediction period for varying inspection interval. It is clear that, for a longer inspection interval, the probability of the bridge component being in the 'as new' condition falls. For a short inspection interval, any changes in the element condition will be detected and the repair happens almost immediately, thus the component is more likely to reside in the 'as good as new' state throughout its life.

The effects of different servicing interval on the condition of the bridge deck can also be seen in Figure 5.37. As expected, a longer servicing interval results in a slightly increase deterioration rate, hence there is a slight reduction in the probability of the bridge deck being in the 'as new' condition. These effects of servicing and inspection intervals are also observed in other bridge components and they show a very similar results with what have been demonstrated using the Markov bridge model (Chapter 4 - 4.3.7).

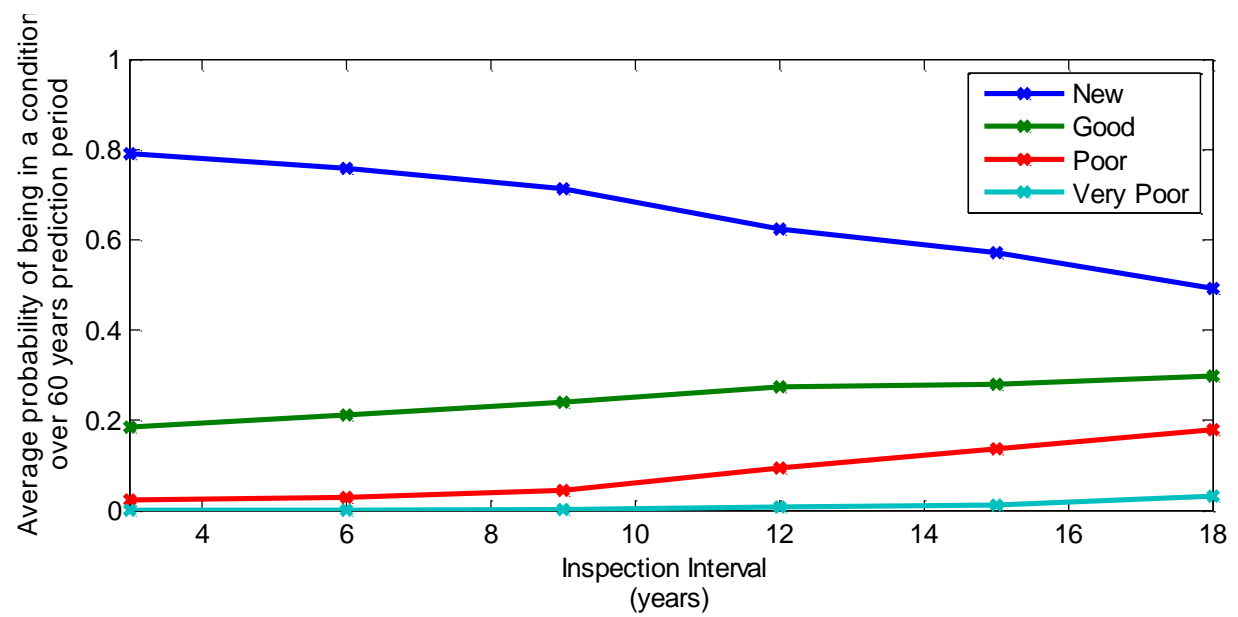

Figure 5.36: Average probability of being in each condition state over 60 years prediction period against different inspection intervals - bridge deck - maintenance strategy: repair as soon as possible. 


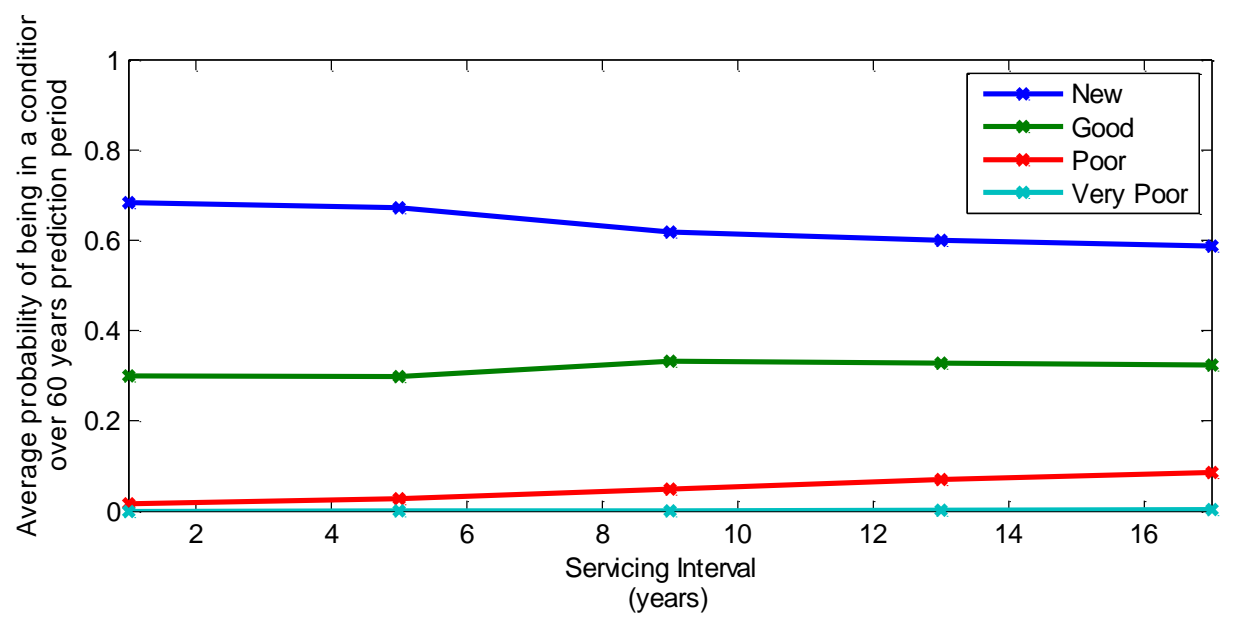

Figure 5.37: Average probability of being in each condition state over 60 years prediction period against different servicing intervals - bridge deck - maintenance strategy: repair as soon as possible.

\subsubsection{Expected maintenance costs}

Statistics collected in section 5.5.4 allow the calculation for the expected maintenance costs to be directly deduced. The estimated unit cost for each intervention type was given in Chapter 3 - 3.6.4, multiplying this figure with the predicted number of intervention, the expected WLCC over the 60 years prediction period for a component can be found. Table 5.10 shows the expected maintenance costs for four maintenance strategies that have been demonstrated throughout this chapter, including three strategies with opportunistic maintenance enabled. These strategies were given in section 5.5.4 and are applied to all of the modelled bridge components. It can be seen that strategy 2, which inhibits minor repair, is the most expensive option. This is because the cost of major repair is significantly (about 3 to 5 times) more than the cost of minor repairs, thus intervention strategies 1 and 3 which allow minor intervention would result in a smaller WLCC. Strategy 4 presented the lowest costs when the components are allowed to deteriorate to very poor conditions. Some of the component's life of reaching the very poor state is long e.g. main girders, abutments, it is expected that these components will not be replaced within the 60 years prediction period, therefore, a low WLCC is predicted.

Strategies with opportunistic maintenance enabled have similar predicted WLCC comparing with corresponding strategies with no opportunistic repair. Although with strategy 2, a significant saving can be seen by carrying opportunistic repairs on the external 
main girders and bearings. The opportunistic costs are reflected in the cost of minor repairs for these components, offsetting these costs against the saving in the major repair costs, the predicted WLCC is actually cheaper by $9.3 \%$ with opportunistic maintenance.

\begin{tabular}{|c|c|c|c|c|c|c|c|c|c|c|}
\hline Strategy & Intervention type & DCK & MGE1 & MGI1 & MGE2 & BGL1 & BGL2 & ABT1 & ABT2 & Total \\
\hline \multirow{4}{*}{1} & Minor repair & 7455 & 16911 & 11024 & 17106 & 6615 & 7336 & 5423 & 5562 & \\
\hline & Major repair & 3174 & 835 & 23861 & 1432 & 32272 & 32951 & 0 & 0 & \\
\hline & Renewal & 0 & 0 & 0 & 0 & 12000 & 8600 & 0 & 0 & \\
\hline & Total & 10629 & 17746 & 34885 & 18537 & 50887 & 48887 & 5423 & 5562 & 202106 \\
\hline \multirow{4}{*}{2} & Minor repair & 0 & 0 & 0 & 0 & 0 & 0 & 0 & 0 & \\
\hline & Major repair & 14689 & 28037 & 41876 & 28872 & 43935 & 45747 & 4150 & 5321 & \\
\hline & Renewal & 2727 & 0 & 0 & 0 & 26400 & 30000 & 0 & 0 & \\
\hline & Total & 17417 & 28037 & 41876 & 28872 & 70335 & 75747 & 4150 & 5321 & 281303 \\
\hline \multirow{4}{*}{3} & Minor repair & 6631 & 16878 & 98 & 17171 & 5873 & 5938 & 5898 & 6373 & \\
\hline & Major repair & 0 & 0 & 0 & 0 & 0 & 0 & 0 & 0 & \\
\hline & Renewal & 6399 & 0 & 3536 & 0 & 58600 & 60400 & 0 & 0 & \\
\hline & Total & 13030 & 16878 & 3633 & 17171 & 64473 & 66338 & 5898 & 6373 & 203344 \\
\hline \multirow{4}{*}{4} & Minor repair & 0 & 0 & 0 & 0 & 0 & 0 & 0 & 0 & \\
\hline & Major repair & 0 & 0 & 0 & 0 & 0 & 0 & 0 & 0 & \\
\hline & Renewal & 27589 & 416 & 2912 & 1248 & 81200 & 80400 & 0 & 0 & \\
\hline & Total & 27589 & 416 & 2912 & 1248 & 81200 & 80400 & 0 & 0 & 203315 \\
\hline \multirow{4}{*}{$\begin{array}{l}2 \text { with } \\
\text { opportunistic } \\
\text { maintenance }\end{array}$} & Minor repair & 0 & 9789 & 3967 & 9561 & 1397 & 1812 & 587 & 671 & \\
\hline & Major repair & 14364 & 11692 & 33047 & 13601 & 44954 & 45181 & 4150 & 4257 & \\
\hline & Renewal & 3776 & 0 & 0 & 0 & 21200 & 21600 & 0 & 0 & \\
\hline & Total & 18140 & 21480 & 37015 & 23162 & 67551 & 68593 & 4737 & 4927 & 255156 \\
\hline \multirow{4}{*}{$\begin{array}{c}3 \text { with } \\
\text { opportunistic } \\
\text { maintenance }\end{array}$} & Minor repair & 6944 & 17333 & 293 & 17138 & 6572 & 6353 & 5702 & 6066 & \\
\hline & Major repair & 0 & 0 & 119 & 0 & 5888 & 4416 & 0 & 0 & \\
\hline & Renewal & 7763 & 0 & 4575 & 208 & 51000 & 53000 & 0 & 0 & \\
\hline & Total & 14706 & 17333 & 4987 & 17346 & 63460 & 63769 & 5702 & 6066 & 202920 \\
\hline \multirow{4}{*}{$\begin{array}{c}4 \text { with } \\
\text { opportunistic } \\
\text { maintenance }\end{array}$} & Minor repair & 0 & 0 & 0 & 0 & 589 & 415 & 0 & 0 & \\
\hline & Major repair & 0 & 954 & 358 & 835 & 8832 & 6794 & 0 & 0 & \\
\hline & Renewal & 27799 & 208 & 2288 & 624 & 72000 & 75600 & 0 & 400 & \\
\hline & Total & 27799 & 1162 & 2646 & 1459 & 81422 & 82809 & 0 & 400 & 207247 \\
\hline
\end{tabular}

Table 5.10: Expected WLCC for all bridge components for four maintenance strategies

Figure 5.38 shows the WLCC for each component under the four maintenance strategies, the plots also show the contribution of the expected costs for each intervention types. Similar information is illustrated in Figure 5.39 for strategies 2-4 with opportunistic maintenance. The plots clearly reflect the intention of the intervention strategies, for example, Figure 5.38(c) shows zero expected costs for major repair, this is because strategy 3 
only considers minor repair and replacement. The plots also shows that, in all the strategies, the bearings, that are already in the poor conditions, would contribute a large proportion to the total maintenance cost, whereas the expected maintenance costs for the abutments are inconsiderable.

(a) Strategy 1
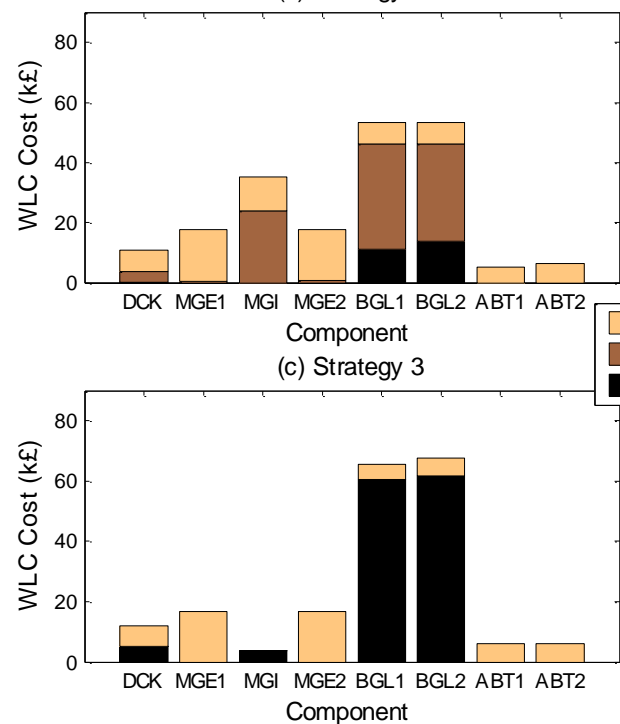

(b) Strategy 2
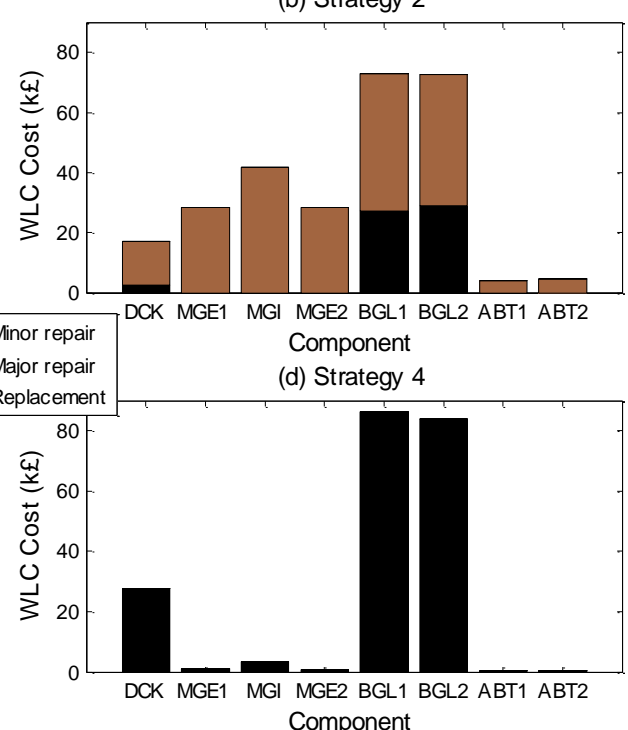

Figure 5.38: Expected WLCC for each bridge components under four maintenance strategies

(a) Strategy 2 with opportunistic maintenance

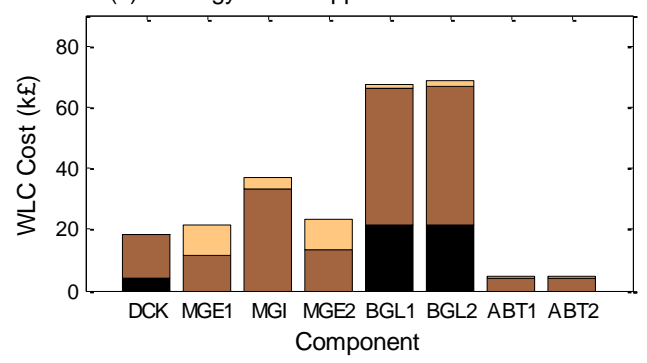

(c) Strategy 4 with opportunistic maintenance

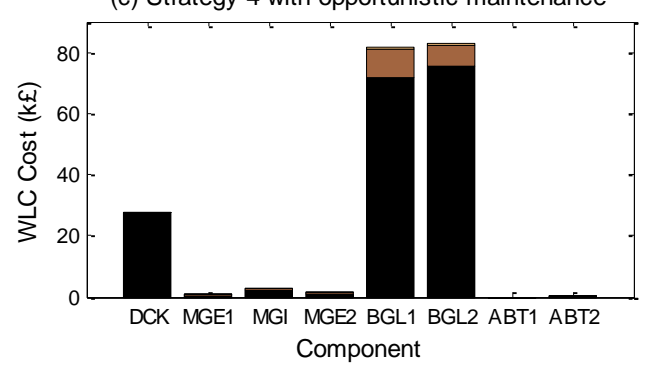

(b) Strategy 3 with opportunistic maintenance

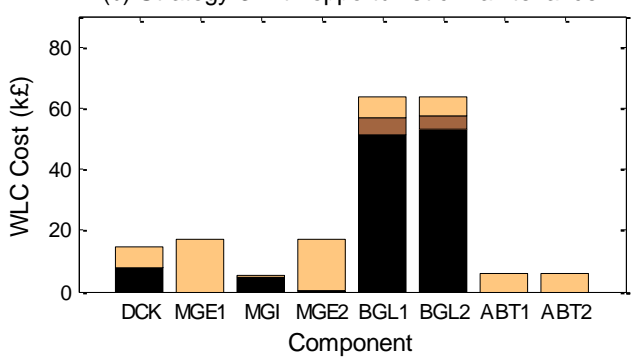

Figure 5.39: Expected WLCC for each bridge components for strategies 2-4 with opportunistic maintenance 


\subsection{Summary}

This chapter described the development of a bridge model using the Petri-Net modelling technique. The technique is increasingly being used in modelling dynamic systems, and has never been applied to model bridge assets. This chapter gives an overview of the PN method before developing a PN bridge model. It also discusses, in detail, the modifications to the original PN modelling technique to suit the problem in modelling bridge asset management. The model is then applied to the selected asset and simulation results are presented and discussed.

The model inputs are the distributions of times that an element degrades to a certain condition state. A Monte Carlo simulation was used to simulate the model and statistics are then collected to show the performance of bridge elements. The PN bridge model developed is considered novel and it overcomes several limitations in other bridge models based on Markov modelling. In particular, the PN bridge model presented several advantages, they are:

- Non-constant deterioration rate,

- Detail modelling of the component, e.g. model the coating of metal component as well as the component itself,

- Repair happens according to a specific maintenance schedule set for a route (depends on the route criticality, the number of planned maintenance block for a year is usually limited),

- Certain types of repairs are ineffective after a certain number of times carried out,

- Modularity properties of the PN model allow other type of assets to be incorporated for across asset modelling. 


\section{Chapter 6 - Markov and Petri Net Model Comparison}

\subsection{Introduction}

In the previous two chapters, two different bridge models have been developed based on the Markov and the Petri-Net techniques. The Petri-Net bridge model presented several advantages that have addressed the limitations in the Markov bridge model. The PN model not only offers a different approach to the modelling of bridge assets and but also has the capability to incorporate more detail. In this chapter, the results obtained by the two modelling approaches are compared and discussed by applying the same maintenance policy on the same selected asset.

\subsection{Predicting component future average condition}

It is expected for the same asset, the average future asset condition profile should be similar using the two models. Given that the model inputs are the same and the same maintenance policy is applied. In this section, the results obtained from the models are investigated to establish if they indicate good matches.

Figure 6.1 shows the predicted condition profile for the bridge deck using the Markov model under a maintenance policy of replacing only when the bridge component reaches the very poor condition. The plot is obtained by progressing from the initial condition in very small time steps over the 60 years prediction 
period. This numerical time-stepping routine means that the model would reach the steady state after a certain time. This can be clearly seen in the plot after 30 years. The 'wave' profile in the plot every 6 years represents the repair and deterioration processes between two inspections. Therefore, in order to calculate the average probabilities of being in each condition states of the bridge deck over 60 years, the calculation should account for the steady-state and the wave profile of the numerical solutions. Thus, these average probabilities are achieved by calculating the moving average of the probabilities shown in the plot with a step size (duration) of 6 years (equals to the inspection period). These values are tabulated in Table 6.1.

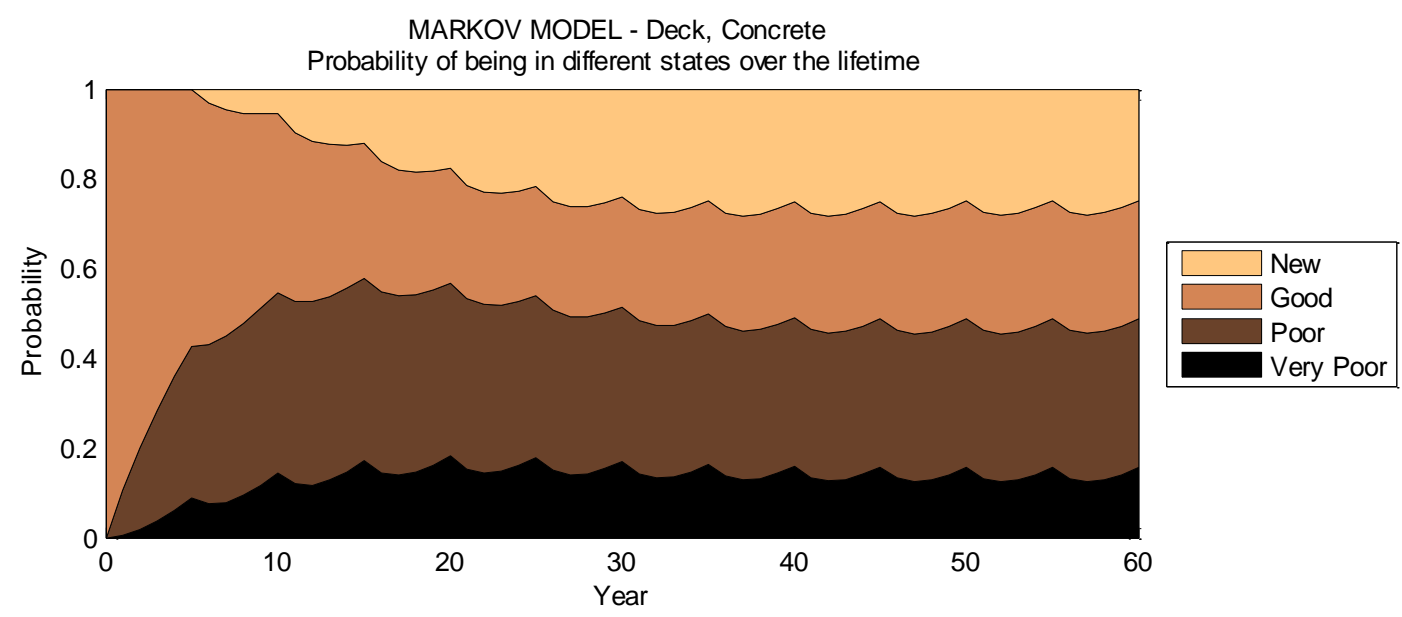

Figure 6.1: Steady-state probabilities of being in different conditions for the bridge deck - Markov model - maintenance strategy is to replace only

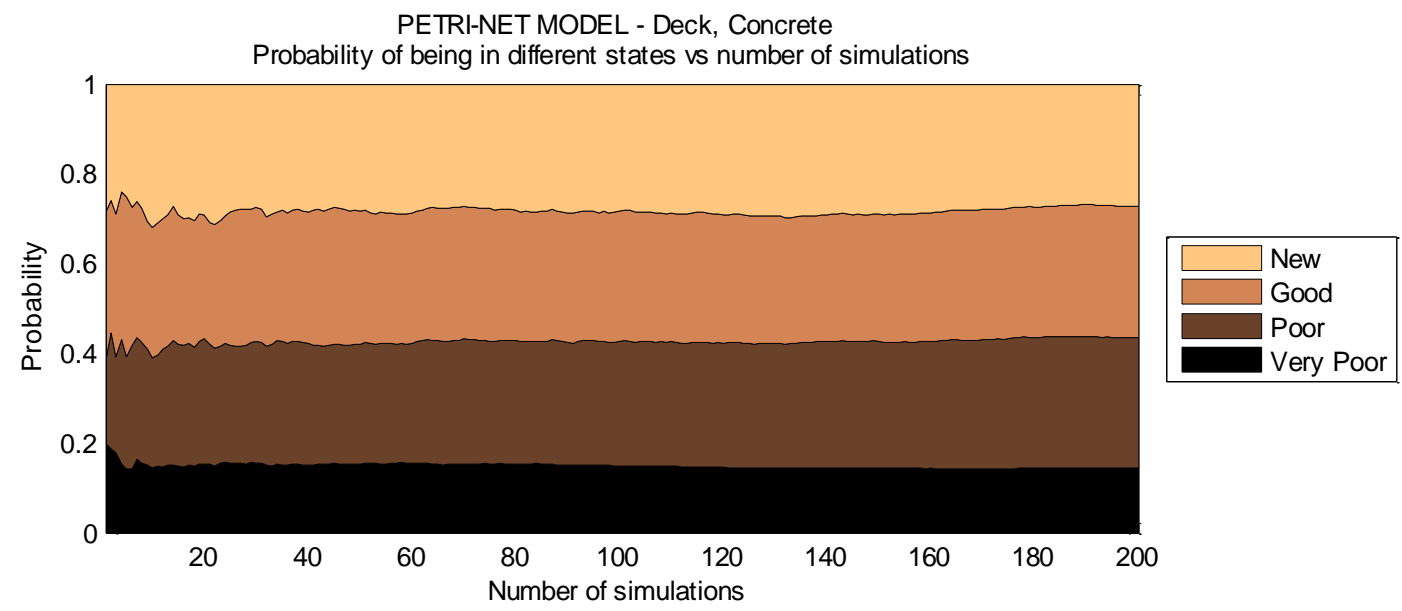

Figure 6.2: Converged probabilities of being in different conditions for the bridge deck - PN model - maintenance strategy is to replace only

For the PN bridge model, the average probabilities of being in each condition are obtained by running the model and looking at the proportion of time the element spending in each state for 60 
years period. The model is then simulated many times and as the number of simulations increases, the average probabilities will converge. Figure 6.2 shows the converged probabilities after 200 simulations. These values are also shown in Table 6.1.

\begin{tabular}{|c|c|c|c|c|c|c|c|c|c|}
\hline & & DCK & MGE1 & MGI & MGE2 & BGL1 & BGL2 & ABT1 & ABT2 \\
\hline \multirow{4}{*}{$\begin{array}{c}\text { Markov } \\
\text { model } \\
\text { results }\end{array}$} & New & 0.2652 & 0.0924 & 0.1359 & 0.0924 & 0.4767 & 0.4767 & 0.4027 & 0.4027 \\
\hline & Good & 0.2641 & 0.2109 & 0.1120 & 0.2109 & 0.1316 & 0.1316 & 0.4631 & 0.4631 \\
\hline & Poor & 0.3303 & 0.6685 & 0.7209 & 0.6685 & 0.2520 & 0.2520 & 0.1305 & 0.1305 \\
\hline & V Poor & 0.1404 & 0.0282 & 0.0312 & 0.0282 & 0.1397 & 0.1397 & 0.0037 & 0.0037 \\
\hline \multirow{4}{*}{$\begin{array}{c}\text { Petri-Net } \\
\text { model } \\
\text { results }\end{array}$} & New & 0.2714 & 0.0020 & 0.0061 & 0.0000 & 0.3521 & 0.3544 & 0.5935 & 0.5831 \\
\hline & Good & 0.2938 & 0.4119 & 0.0033 & 0.4176 & 0.0975 & 0.1019 & 0.3618 & 0.3835 \\
\hline & Poor & 0.2911 & 0.5833 & 0.9793 & 0.5816 & 0.3053 & 0.2975 & 0.0447 & 0.0333 \\
\hline & V Poor & 0.1436 & 0.0028 & 0.0113 & 0.0008 & 0.2451 & 0.2461 & 0.0000 & 0.0000 \\
\hline
\end{tabular}

Table 6.1: Average probability of being each state over the whole predicted life for all modelled bridge components - maintenance strategy is to replace only

Figure 6.3 best illustrates the differences in the results by plotting, for each bridge element, the values obtained for the Markov model using one column (first column). The second column shows the results of the PN model. For the bridge deck, the average probabilities of being in each state are very similar. The biggest differences are in the probabilities of being in the good and the poor state. The Markov model predicted an average probability of being in the poor condition of $4 \%$ higher than that predicted by the PN model. For other bridge components, the results did not indicate good matches. The reason for this is actually because of the prediction period set in the PN model. In the Markov model, the steady state of the system can be quickly achieved after several time steps. However, for the PN model, since the model is solved by simulation, the steady state can only be achieved when the simulation time and the number of simulation is set to long enough. Now since the mean life to 'failure' of the bridge components are quite long, some of the components will even remain in the same state over the 60 years prediction period. Therefore in order to obtain the steady-state average probabilities using the PN model, and to make the results from the two models comparable, the prediction period in the PN model was set to 200 years. This is to ensure the model prediction time fully covers the life of the components. The results obtained are then plotted again and shown in Figure 6.4. It shows very good agreements in the results between the two models with the differences in the results are mostly less than $10 \%$ in 
probability. A large difference of around $20 \%$ probability can still be observed in the prediction for the future probability of being in the good and the poor state of the bridge abutments. Again it is because the complete life of the abutments is very long (with the mean time to replacement is around 150 years). It is expected that for an even longer prediction time, the differences will be lowered. The result is not illustrated here since it is unnecessary to run the model for longer prediction period.

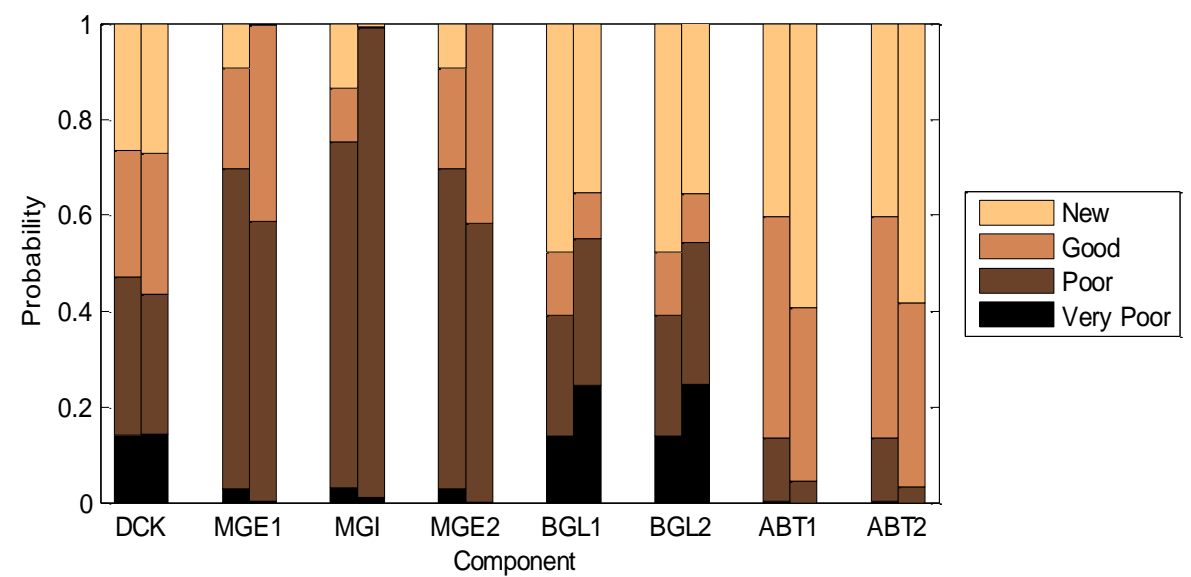

Figure 6.3: Average probabilities of being in each condition states for all bridge components. The $1^{\text {st }}$ and $2^{\text {nd }}$ columns show the results obtained from Markov and Petri-Net bridge model respectively. PN model prediction period is 60 years.

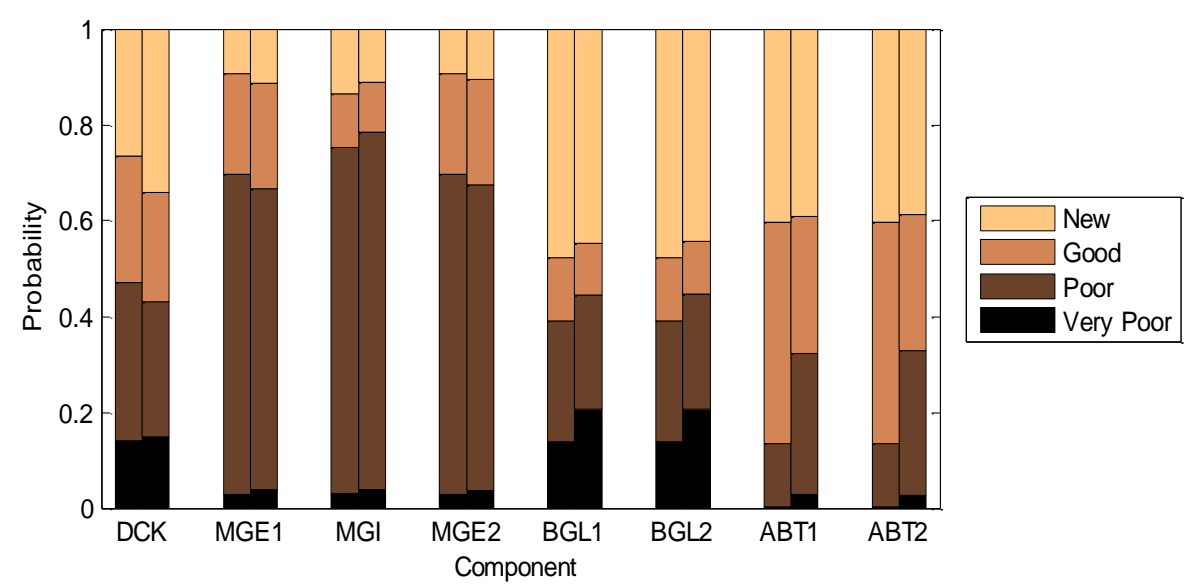

Figure 6.4: Average probabilities of being in each condition states for all bridge components. The $1^{\text {st }}$ and $2^{\text {nd }}$ columns show the results obtained from Markov and

Petri-Net bridge model respectively. PN model prediction period is 200 years.

More importantly, the PN model incorporates more detail with the consideration of several factors that affects the deterioration process of bridge elements. Therefore, these would always be small differences in the models results which these factors are accounted for. In particular, these factors are: the increasing deterioration rates of bridge elements, the degradation of 
protective coatings for metal elements and the limit of the effectiveness of repair.

In general, it can be observed that, the two models predicted very similar future average probabilities of being in each conditions when the two models are in the steady state. Since the Markov model uses a widely adapted modelling technique for modelling bridges, the results validate the propriety of the PN modelling technique used in the PN bridge model in modelling bridge assets.

\subsection{Predicting component future condition profile}

Although the results obtained using the Markov model are good indications of the future average condition profile for some systems. It is believed that, the model's results obtained when the model is in the steady-state might not be useful for forecasting asset condition when they feature a long life time. In this research, the modelled asset has some components with the mean life of up to 100 years. This means that, what the Markov model is effectively indicating is actually the average future condition profile of the components after 100 years. This is also the reason why most of the bridge models available in the literature, based on Markov modelling, have a prediction period of more than 100 years. It is difficult then to predict what is going to happen in the near future as a 100-year-prediction seems unrealistic. In contrast, the PN model, with its simulation modelling nature, provides a clearer and more accurate prediction of the asset condition at any given point in time in the future.

Figure 6.5 shows the predicted condition profile of the bridge deck using the Markov model. Figure 6.6 illustrates the future condition profile using the PN model. In both plots, the bridge deck can be seen as being in the good condition at the start of the simulation period of 60 years. Progressing to the following years, the probability of the deck remaining in this condition decreases and the probabilities of being in the poor and very poor state increase. However, whilst the Markov model shows that the probabilities quickly converge to the steady-state, the PN model shows a much clearer profile. It can be seen that the probabilities of the deck being in the poor and very poor condition gradually increase in the first 25 years. It is predicted that the probability of being in 
these two conditions peaks at $95 \%$ at the year 23 . From this year, this probability starts decreasing and the probability of the deck being in the 'as new' condition rises. This is because associating with the high probability of the deck being in the worse conditions, an intervention should be carried out around this period. The condition profile indicates that this intervention is expected to happen at around between the $20^{\text {th }}$ and $35^{\text {th }}$ year. After the intervention, when the component condition is restored, the component's deterioration process starts again. This is reflected in the plot from 35 to 60 years, with the decreasing probability of being in the 'as new' condition and the increasing probabilities of being in worse conditions.

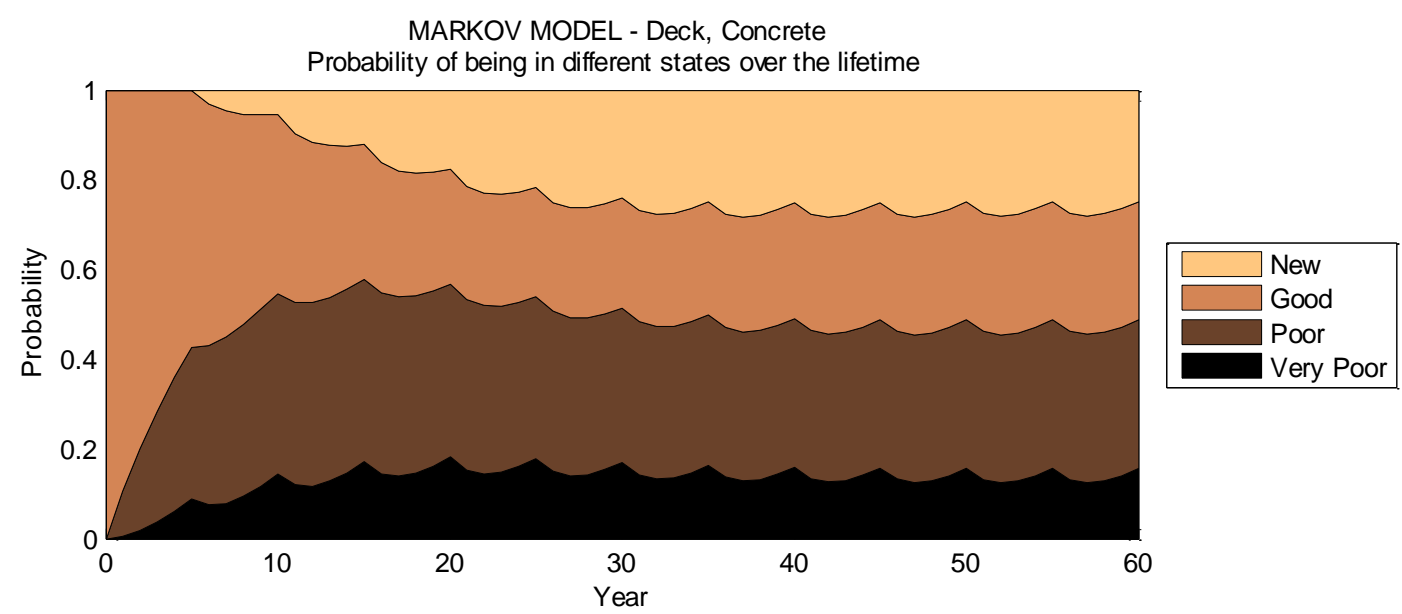

Figure 6.5: Predicted future condition profile for the bridge deck - Markov model maintenance strategy is to replace only

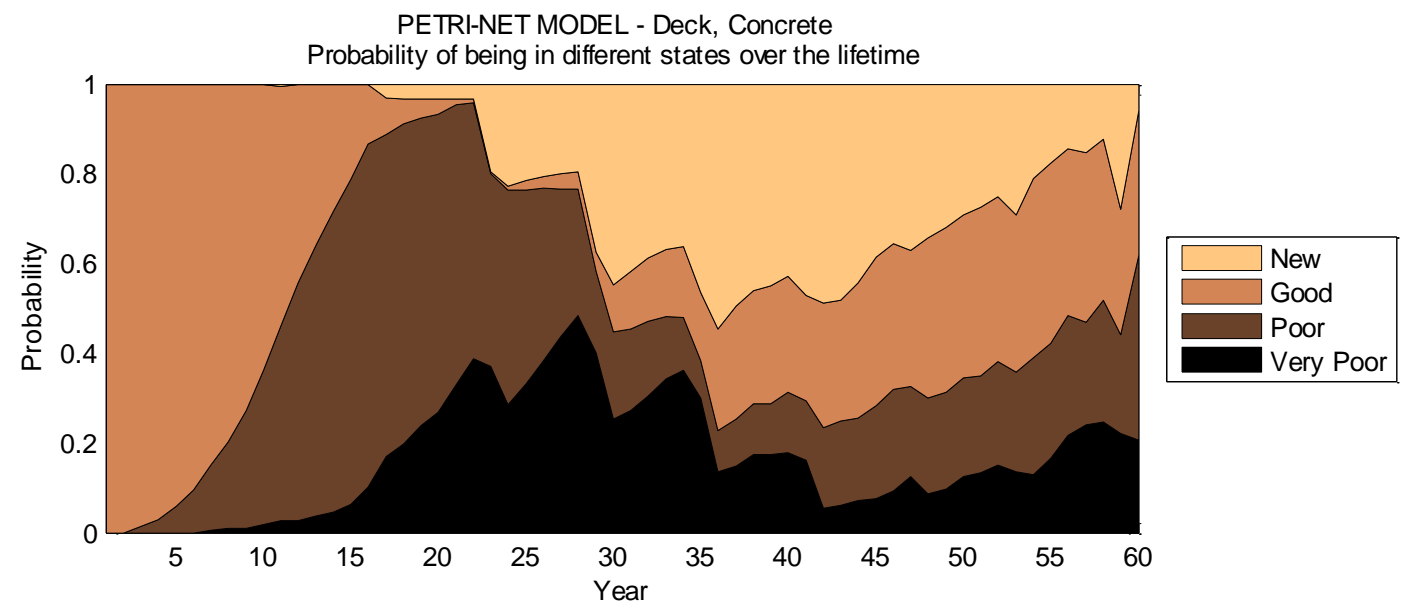

Figure 6.6: Predicted future condition profile for the bridge deck - PN model maintenance strategy is to replace only 

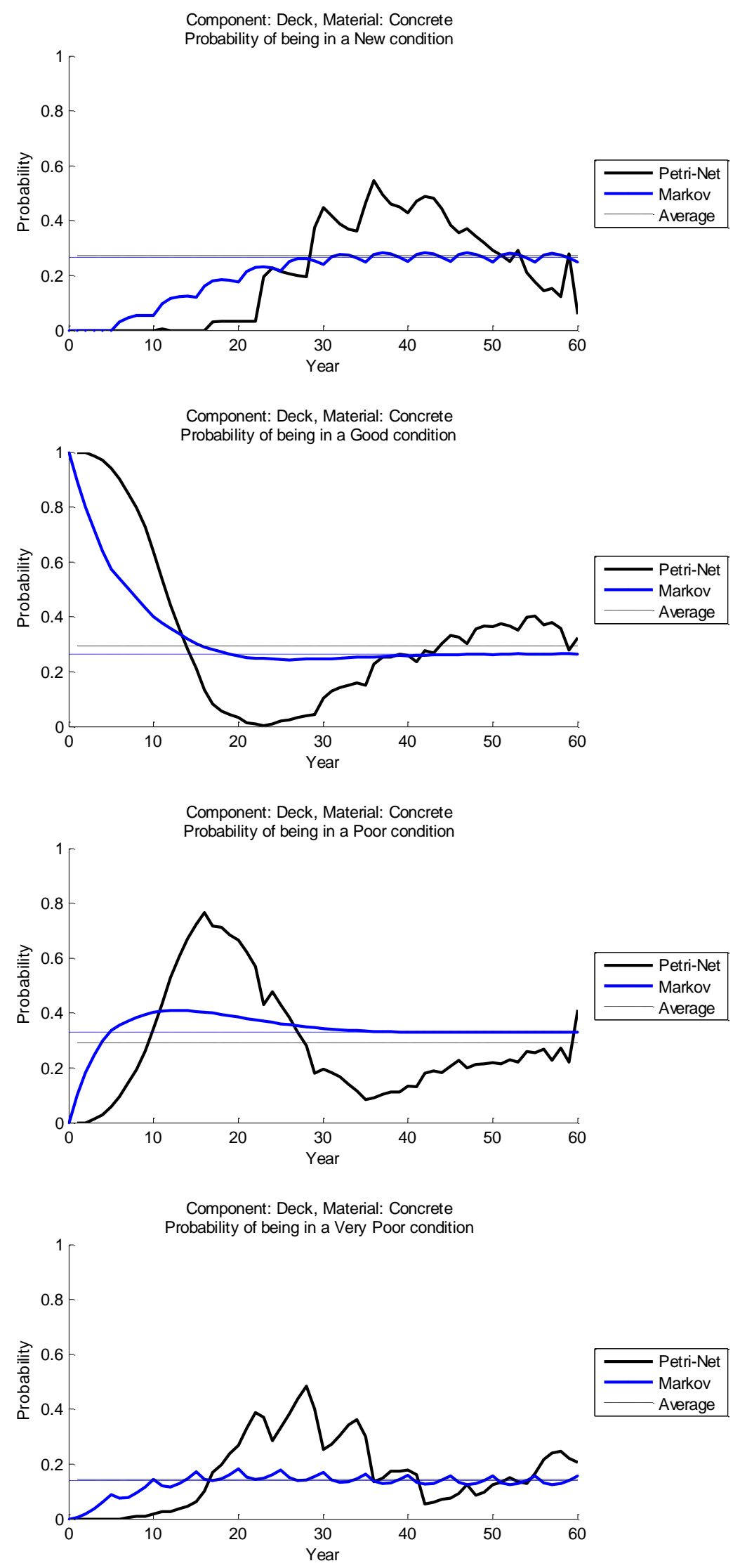

Figure 6.7: Comparing the predicted probabilities of being in the 'as new', good, poor and very poor condition using the Markov and PN models. The dash line shows the average probabilities of being in these conditions over the whole 60 years prediction period. 
In more detail, the plots in Figure 6.7 compare the predicted probabilities, by the two models, of being in each of the four conditions individually. The plot also shows the average probabilities over the 60 years prediction period. It is observed that, although the condition profiles are different, the average probabilities are very similar as was discussed in the previous section. The PN model simulates the deterioration process using the times sampled from the degradation distribution obtained by studied historical data. Therefore with the characteristic life of the bridge deck to be replaced from the good condition is around 23 years, this is shown in the $4^{\text {th }}$ plot with the peak probability of being in the very poor condition around this year.

In conclusion, the PN model offers not only a more accurate prediction of the near future condition profile, but also allows more information to be extracted from the model that can be used to support the maintenance decision making process. For example, it is able to show the period in the asset life time, where it is likely that an asset will be in a certain condition that triggers an intervention. Maintenance attentions and resources can then be distributed accordingly over the component life time.

\subsection{Predicting asset future average condition}

Figure 6.8 and 6.9 show the average asset condition under 3 different intervention strategies as predicted by the Markov and the Petri-Net models respectively. These 3 policies were already discussed in Chapter $4-4.2 .1 .3$. It can be seen in both graphs that the average asset initial condition is just below a poor condition. Looking at the prediction under strategy 1 , during the first 6 years until the first inspection, the asset condition gradually drops as the asset degrades. After the first inspection, the asset condition rises to just under good condition indicating the effect of maintenance. In both models, repairs are associated with scheduling times of between 1 to 3 years depending on which type of repairs. This is reflected in the rise in the asset condition predicted by the PN model at around the $8-9^{\text {th }}$ year which is a few years after the first inspection. However, it can be seen that the rise happens earlier in the Markov model prediction at the $6^{\text {th }}$ year and the repair delay period is not observed in the plot. This is because the Markov model actually reflects different 
repair scheduling times by altering the repair rates, while the instantaneous shift in the probabilities always happen after every 6 years period. Therefore, the 'wave' profile seen in Figure 6.8 has a 'wave length' of exactly 6 years period, whereas the 'wave length' in Figure 6.9 is longer.

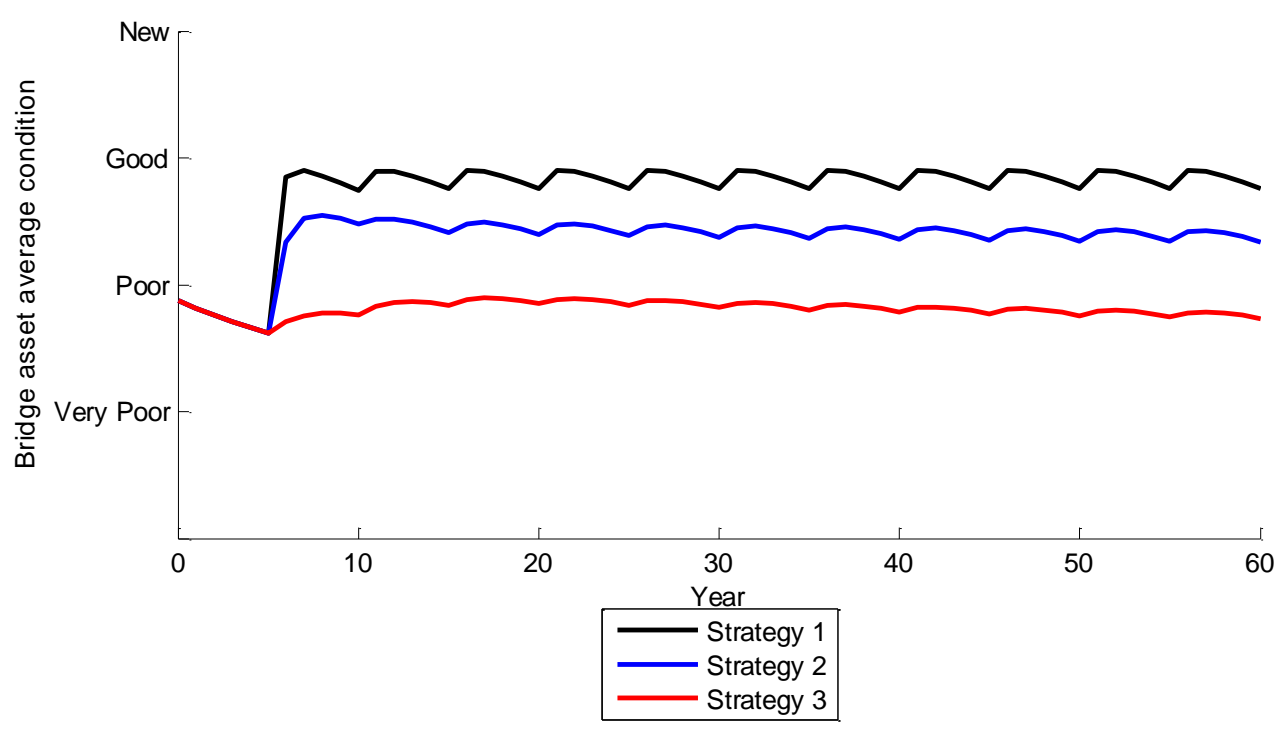

Figure 6.8: Predicted average asset condition under different maintenance strategies using Markov model

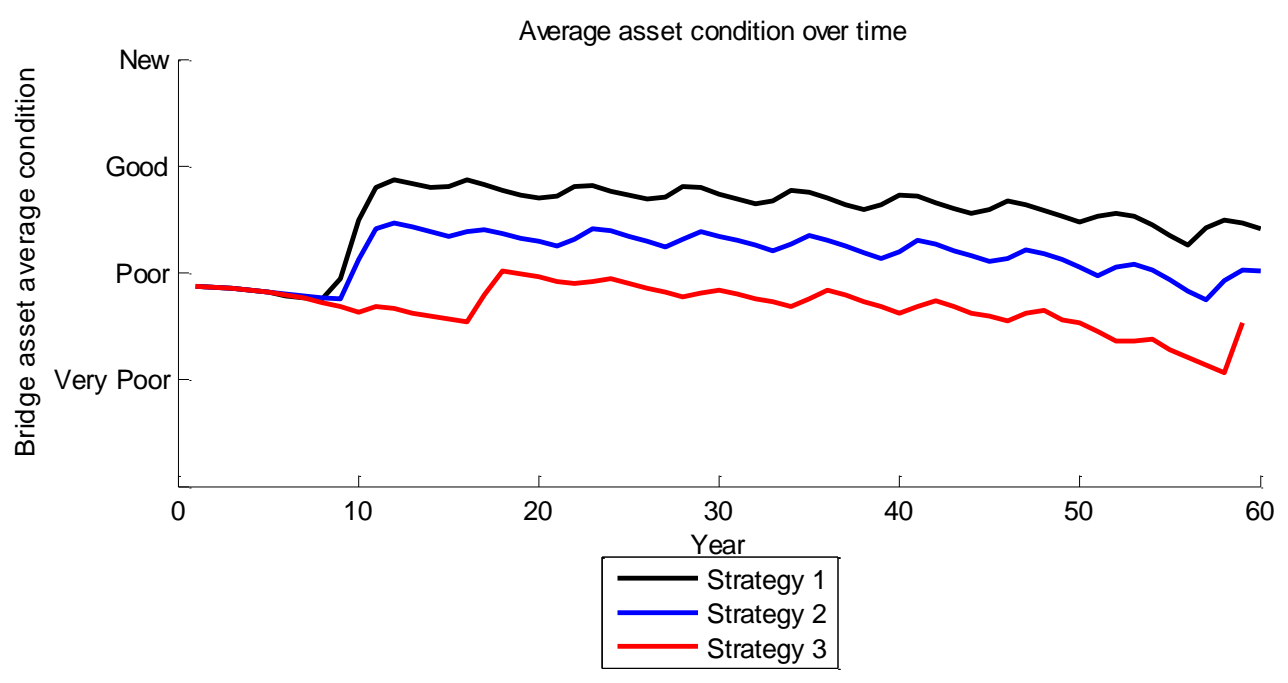

Figure 6.9: Predicted average asset condition under different maintenance strategies using Petri-Net model

What can also be observed in the PN model prediction is the degrading trend of the asset condition over the whole life cycle. The decreasing in the average asset condition is caused by the increasing probability of the bridge components being in worse 
states as the prediction time progresses. In both models, the repair is assumed to be perfect i.e. the condition of the component is restored to 'as good as new' at repair. This means that for strategy 1 and 2, as long as the inspection is carried out frequently enough so that minor and major interventions can be carried out before the component reaches the very poor state, the component will never have to be replaced. This is implied in the Markov model prediction as strategy 1 and 2 would maintain the asset condition at a constant condition level. In the PN model, the introduction of a limit to the number of effective repairs means that once the number minor and major interventions has been reached, the component has no choice but to degrade to the very poor state where it is renewed. This results in an increase of the probability of the components being in worse states as the number of interventions (minor and major repair) increases, hence the asset average condition gradually declines.

Strategy 3 allows the component to deteriorate until complete replacement. Because different components have different times to degrade which are, in many cases, longer than 60 years prediction period, some components would be replaced whilst other components are still degrading to the very poor state. Since the average asset condition is calculated as the average of all component conditions, the degradation of one component would also reflect in the degradation of the whole asset condition. Therefore, the degrading trend of the asset under this strategy can be seen in both models.

\subsection{Predicting maintenance cost}

Table 6.2 contains the predicted whole life cycle costs (WLCC) for each of the modelled bridge elements under three different intervention strategies. As discussed earlier, with the Markov model having constant deterioration rates, it doesn't account for the wear out effects of bridge components as does the PN model. Thus the Markov model tends to underestimate the probabilities of the bridge components being in worse states (poor and very poor state), this results in a slightly smaller estimations of the WLCC predicted by the Markov model comparing with the PN model for strategy 2 and 3 . The underestimation of the probabilities being in the worse states means that the Markov 
model also over estimates the probabilities of being in the 'as new' $^{\prime}$ and the good state, hence the estimation of the WLCC for strategy 1 is actually larger than that predicted by the PN model. As the probabilities of being in different states of bridge components predicted by two models are not exactly the same, the predicted WLCCs will be slightly different. However, the results, as demonstrated in Figure 6.10, still show good agreements with the differences in the predicted total WLCCs for the asset are less than $10 \%$.

\begin{tabular}{|c|c|c|c|c|c|c|c|c|c|c|}
\hline & Strategy & DCK & MGE1 & MGI1 & MGE2 & BGL1 & BGL2 & ABT1 & ABT2 & Total \\
\hline \multirow{3}{*}{ 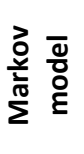 } & 1 & 20786 & 25095 & 39978 & 25095 & 51763 & 51763 & 5669 & 5669 & 235366 \\
\hline & 2 & 23660 & 28807 & 40521 & 28807 & 63611 & 63611 & 3256 & 3256 & 265079 \\
\hline & 3 & 31635 & 8767 & 15396 & 8767 & 51016 & 51016 & 496 & 496 & 177141 \\
\hline \multirow{3}{*}{ 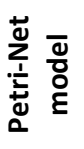 } & 1 & 10629 & 17746 & 34885 & 18537 & 50887 & 48887 & 5423 & 5562 & 202106 \\
\hline & 2 & 17417 & 28037 & 41876 & 28872 & 70335 & 75747 & 4150 & 5321 & 281303 \\
\hline & 3 & 27589 & 416 & 2912 & 1248 & 81200 & 80400 & 0 & 0 & 203315 \\
\hline
\end{tabular}

Table 6.2: WLCC for each bridge components under different maintenance strategies as predicted by the two bridge models
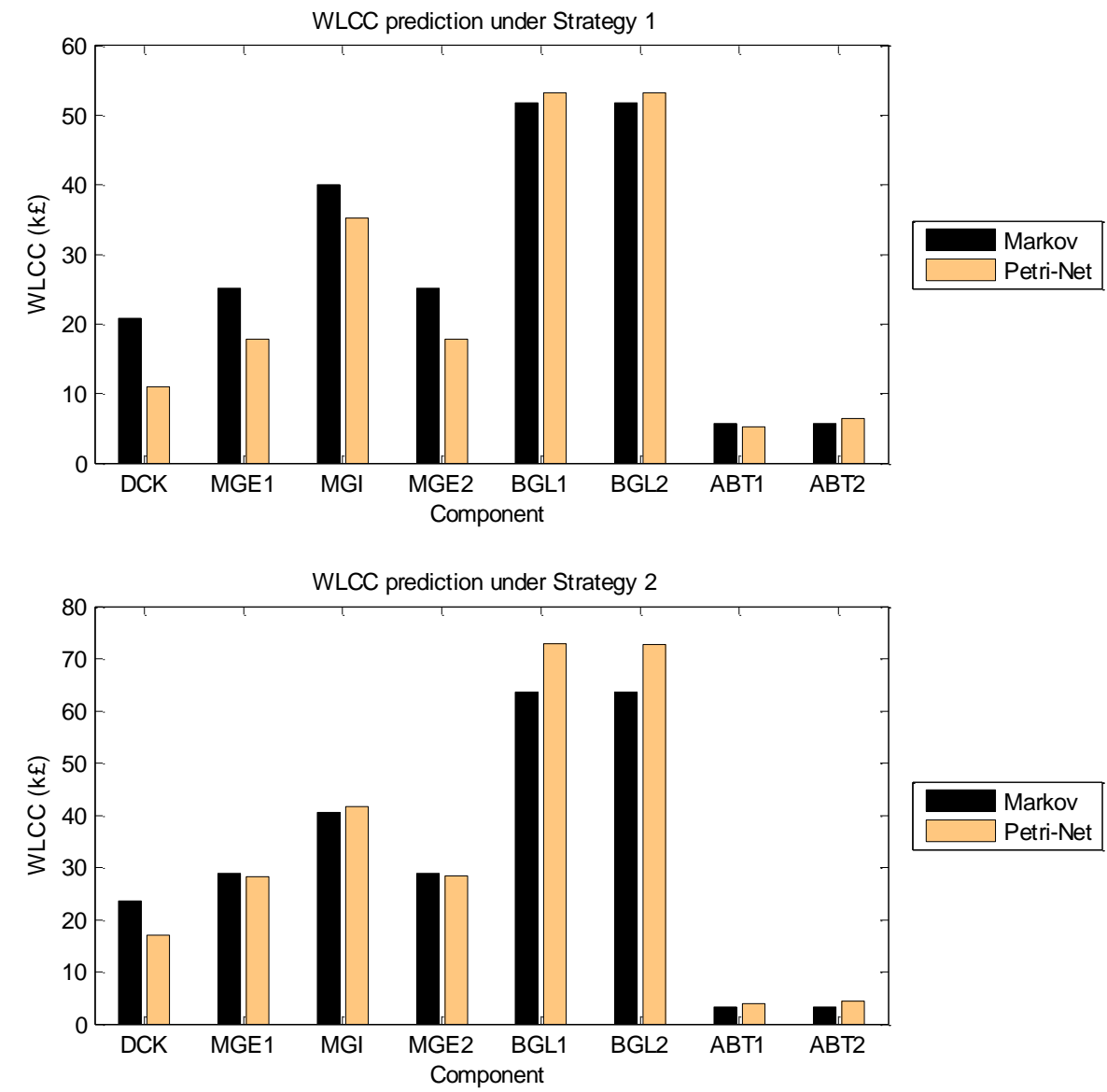


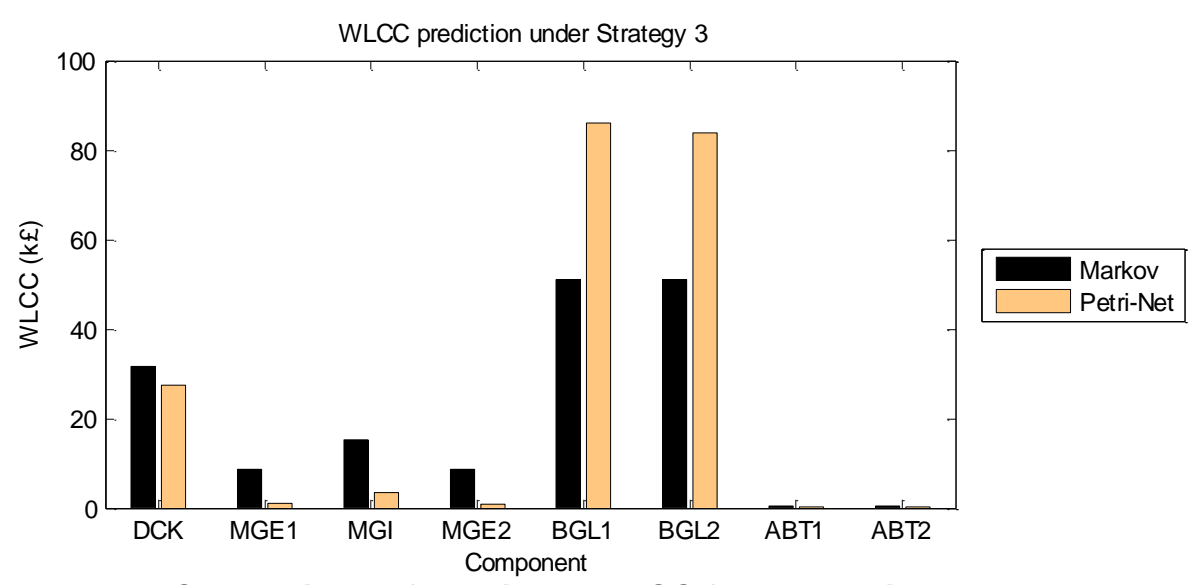

Figure 6.10: Comparison of predicted WLCC for each bridge components under different maintenance strategies

\subsection{Model performances}

In the Markov model, the model size depends on the number of the modelled components. With the four condition states considered for each component in the model, the model size increases exponentially to the base of 4 with the exponent being the number of the components. The most time consuming task in solving the Markov model is in the generation of the transition matrix and the numerical solution to find the transient solution. For the PN model, the model size actually stays the same, however, more tokens are added that represent each added elements. The most time consuming task in the PN model solution is the number of simulations requires for the solution to converge. Table 6.3 compares the solution times of the models with the increasing number of modelled bridge components. Note that the PN model was run for 200 simulations in all exercises.

It is obvious that with the increasing number of modelled elements, the solution time increases. The Markov model is considered efficient for a bridge model contains 8 elements or fewer. The solution time increases dramatically when there are more than 8 elements, and when the number of modelled elements is 20 or more, the solution time becomes very large and could not be obtained. The solution time for the PN model increases linearly with the number of the components with the average solution time for one component is around 4 seconds. The PN model is clearly more efficient with the average simulation and solution time of around 22 minutes for a system of 50 components. The solution of the PN model is achieved by Monte 
Carlo simulation, therefore with parallel computing, the solution time can be greatly reduced. The times obtained here are actually achieved by the use of a two-core machine, thus the solution time would be almost double the time presented, if the model is run on a single core machine. It is realised that the model time also depends on the efficiency of the code and algorithm that was used to program the model. However, it can be demonstrated that the Markov model is unsuitable for large systems, whereas, the PN model is clearly more efficient.

\begin{tabular}{|c|c|c|c|c|}
\hline \multirow{2}{*}{$\begin{array}{c}\text { Number of } \\
\text { components } \\
\text { modelled }\end{array}$} & $\begin{array}{c}\text { Number of } \\
\text { tokens in the } \\
\text { model }\end{array}$ & $\begin{array}{c}\text { Solution time } \\
(\mathbf{s})\end{array}$ & $\begin{array}{c}\text { Number of } \\
\text { states in the } \\
\text { model }\end{array}$ & $\begin{array}{c}\text { Solution time } \\
\text { (s) }\end{array}$ \\
\hline 1 & 1 & 4.16 & 8 & 0.01 \\
\hline 2 & 2 & 8.20 & 32 & 0.02 \\
\hline 3 & 3 & 13.20 & 128 & 0.20 \\
\hline 4 & 4 & 32.93 & 512 & 0.67 \\
\hline 5 & 5 & 55.53 & 2048 & 2.36 \\
\hline 6 & 6 & 75.20 & 8192 & 4.07 \\
\hline 7 & 7 & 81.12 & 32768 & 7.58 \\
\hline 8 & 8 & 84.04 & 131072 & 42.69 \\
\hline 9 & 9 & 88.43 & 524288 & 313.91 \\
\hline 10 & 10 & 95.00 & 2097152 & 3323.68 \\
\hline 20 & 20 & 166.14 & $2.19902 \mathrm{E}+12$ & - \\
\hline 50 & 50 & 1305.15 & $2.5353 \mathrm{E}+30$ & - \\
\hline
\end{tabular}

Table 6.3: Comparison of model size and solution time for increasing number of modelled bridge components

\subsection{Summary}

This chapter compared the two developed bridge models in term of model results and performances. Since the Markov modelling technique is widely used for modelling the condition of bridges under different maintenance regimes, by comparison with the Markov model, the PN model results can be validated. The results confirmed that both models predicted very similar future average component conditions. This is reflected by studying the predicted probabilities of bridge elements being in each conditions when the models reach steady-state. Thus, the PN modelling technique is considered suitable for modelling bridge assets. Furthermore, the PN bridge model is able to predict more realistic and informative asset condition profiles in the near future. This information can then be used to investigate the period where there is a high 
probability of an asset being in a poor or a very poor condition, thus maintenance and inspection can be focused accordingly.

It is also demonstrated that, whilst the Markov model is efficient for small bridge systems, an increase in the number of components considered in the model has a considerable effect on the number of resulting states which significantly increases the solution time. The PN model is more efficient in the modelling time especially with a system contains of many components. Moreover, the nature of the PN model lends itself to parallel computing enabling the simulation time can be decreased using high performance computing. 


\section{Chapter 7 - Bridge Maintenance Optimisation}

\subsection{Introduction}

An important use for models of engineering systems is to establish the parameters which can be selected in order to deliver the 'best' or optimal performance. In particular, for asset management, is the desire to find the best maintenance strategy that would result in the lowest whole life cycle cost or the best asset condition so the risk of service disruption is minimised. The easiest way to find the optimum solution is by exhaustive search. This involves evaluating all possible candidate solutions to find the fittest one. However, most engineering problems are complex in which case the evaluation of each potential solution is a time consuming task. This is especially true if, in addition, there is a large dimensioned search space. These factors prohibit the option to try each solution one by one. Therefore, an optimisation technique is employed in finding the best solutions. The optimisation technique used in this project is the Genetic Algorithms (GA). This technique is chosen because: it is a flexible method capable of solving a wide range of optimisation problems with a diversity of variable and constraint types. The GA is also used because it is a simple concept based on the natural evolution and is easy to understand and apply. Also the interaction between variables in this problem is low so the GA is suitable (Haupt and Haupt, 2004). 


\subsubsection{Genetic Algorithm optimisation}

The Genetic Algorithm (GA), first conceived by Holland (1975), is an optimisation and search technique based on the principles of genetics and natural evolution. Based on Darwin's survival-of-thefittest principles, the GA's intelligent search procedures find the best and fittest design solutions which are otherwise difficult to find using other techniques. The GA is considered one of the most powerful search and optimization algorithms because the GA is conducted using a population of points rather than a single point, thus increasing the exploratory capability of the GA (Samhouri, 2009). In addition, the GA lends itself naturally to implementation in parallel processing, with the potential to achieve faster computational times. The GA works with a direct coding of the parameter set rather than the parameters themselves, so, it is suitable for discontinuous, high dimensional and multi-nodal problems. Overall, the advantages of the GA (Haupt and Haupt, 2004) include:

- Optimising with either continuous or discrete variables,

- Doesn't require derivative information of the objective functions,

- Deals with complex problems of a large number of variables,

- Well suited for parallel computers hence potential faster solution times,

- Produces a set of optimum solutions, not just one optimum solution,

- Technique that can be easily adapted to a wide range of problems in all fields.

There are seven important steps in the GA optimisation process (coding \& decoding, initial population generation, fitness function evaluation, selection, mating and mutation) illustrated in Figure 7.1. At the start of the process, the variables to be optimised and the objective functions must be defined. The objective functions are dependent upon the variables and are used to measure if one solution is better than another. In GA terms, the objective function generates the 'fitness' of an 'individual'. The next step is generating the initial population where the variable value representing each individual in the population is encoded by a binary string. From this population, the fitness of each individual is evaluated. These individuals are then ranked according to their 
fitness value. The part of the population which is fittest is retained in the next generation. The rest of the population is replaced with offspring produced by mating some of the fittest individuals in the current population. Some of the newly generated offspring will be randomly mutated to ensure the algorithm explores a broader search space. The new population will then go through the same process where each individual is now decoded for the objective function evaluation. This process is carried out for a number of generations until the solutions converge i.e. produced very similar fitness values. At this point, a set of possible optimum solutions are obtained.

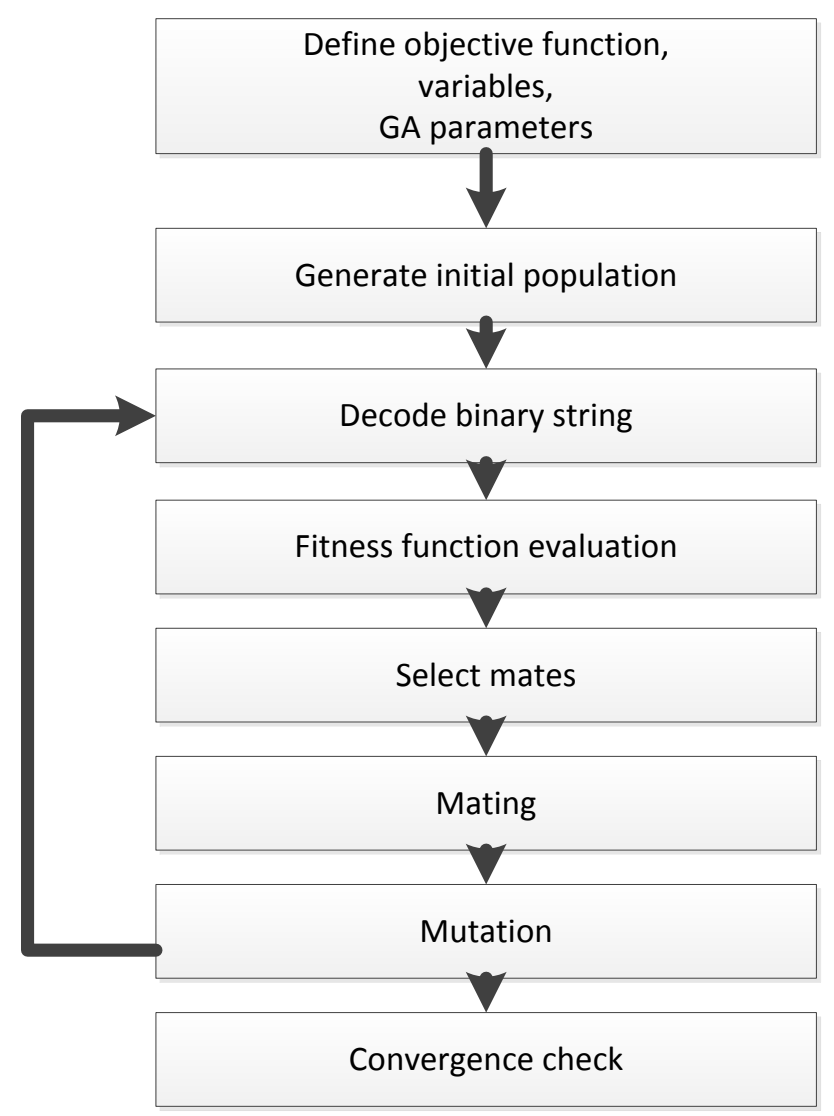

Figure 7.1: Genetic Algorithm optimisation main steps

\subsubsection{Multi-objectives GA (MOGA) optimisation}

The definition of a multi-objective problem (MOGA) is a problem which has two or more, usually conflicting, objectives. The main difference from a single-objective optimisation is that a multiobjective problem does not have one single optimal solution, but instead has a set of optimal solutions, where each represents a trade-off between the objectives. A way to avoid the complexities of multi-objective optimization is to convert the multi-objective 
problem into a single-objective problem by assigning weights to the different objectives and then calculating a single fitness value. The major problem with this weighted sum approach is that it is subjective, as it ultimately leaves it to the decision maker to assign weights according to the subjective importance of the corresponding objectives.

For the modelling purposes of this project, it is of interest to optimise a number of objectives at the same time. A single optimisation exercise was actually applied to find the maintenance policies that result in the lowest life cycle cost (LCC). It was found that, the optimisation usually looks for the strategy that carries out the least number of interventions in order to achieve minimum LCC. This could be undesirable in some cases since these optimum policies, which carry out 'minimum' work, means that the asset condition would be around the critical condition over its life. Moreover, it is believed that the solutions only cover the extreme part of the solution space which might not be meaningful in aiding the maintenance decision making process. Therefore, two objectives are chosen for the optimisation problem in this research which are the asset life cycle cost and the asset average condition. The optimisation exercise is then to find the optimum maintenance strategy that gives the lowest LCC whilst maximising the condition of an asset. The MOGA ranks these objectives according to the Pareto front. The ranking will be discussed in more details later on.

\subsubsection{Optimisation procedure}

There are two bridge models have been developed in this research, based on the Markov and Petri-net method. These two bridge models will be optimised separately and the optimisation process implementation for each model will be discussed in detail. The next sections discuss the optimisation of the Markov and Petri-net bridge models. It is worth noting that, the optimisation of the PN model is a hybrid optimisation, i.e. the results from the optimisation of the Markov model are used as the initial starting points for the PN model optimisation resulting in a performance increase. 


\subsection{Markov bridge model optimisation}

\subsubsection{Objective functions}

The bridge model was developed based on the Markov chain approach described in Chapter 4 . The model simulates the life of all bridge components and the effects of degradation and repair on them. The model can be optimised with respect to many different possible objectives that depend on specific requirements for the asset. For example, if an asset is critical, it may be required to keep the asset in at least a certain condition, the objectives would then be to find maintenance strategies that would maintain the 'best' average condition of the asset whilst achieving the minimum cost. The two objectives selected in this optimisation study are to find maintenance strategies that result in lowest life cycle cost whilst maximising the average condition of the asset. Note that the optimisation is applied to a single asset to demonstrate its capability. The asset selected is again the typical metal underbridge used as an example throughout this thesis. All bridge components modelled are considered to be of equal importance to the structure.

\section{Average condition of the asset}

The average asset condition is a single value calculated by multiplying the average probabilities of being in each condition state with a vector containing a scalar value from 1 to 4 using equation (4.9) as discussed in Chapter $4-4.2 .8$. In the optimisation exercise, the minimisation of this value is the same as maximising the average asset condition. The average condition of the asset was assumed to be the average condition over all major elements modelled. Thus the objective of maximising the average asset condition is achieved by minimising Equation (7.1).

$$
\min \sum_{i=1}^{N} \frac{\left[\begin{array}{llll}
\mathrm{Q}_{1}^{i}(T) & \mathrm{Q}_{2}^{i}(T) & \mathrm{Q}_{3}^{i}(T) & \mathrm{Q}_{4}^{i}(T)
\end{array}\right]}{N} \times\left[\begin{array}{l}
1 \\
2 \\
3 \\
4
\end{array}\right]
$$

where

$N=$ Number of bridge components in a bridge

$T=$ Length of the prediction period

$\mathrm{Q}_{j}^{i}(T)=$ Probability of the component $i$ in state $j$ at time $\mathrm{T}$ 


\section{Life cycle costs}

The life cycle cost (LCC) is calculated as the total expected maintenance cost including the servicing and the inspection costs over the whole prediction period. By summing the LCC for each component modelled, the expected LCC for an asset can be calculated. The objective of minimising the LCC for an asset is achieved using the Equation (7.2).

$$
\begin{aligned}
& \min _{0 \leq t \leq T}\left[\sum _ { i = 1 } ^ { N } \left[\int_{0}^{T} Q_{k}^{i}(t) \cdot v_{1}^{i} d t \times C_{1}^{i}+\int_{0}^{T} Q_{l}^{i}(t) \cdot v_{2}^{i} d t \times C_{2}^{i}+\int_{0}^{T} Q_{m}^{i}(t) \cdot v_{3}^{i} d t \times C_{3}^{i}\right.\right. \\
& \left.\left.\quad+N_{S} C_{S}+N_{I} C_{I}\right]\right]
\end{aligned}
$$

\subsubsection{Variables}

There are a number of variables which can be set in the bridge model, each variable has a range of values and a combination of these variables will form a specific maintenance strategy. Some of the variables are applied to the whole bridge structure whilst some are applied to each bridge component individually. The bridge model allows 6 model variables to be adjusted.

1. Inspection interval (applies to the whole structure)

a. For railway bridges managed by Network Rail, the inspection intervals are set out in Specification 
NR/SP/CIV/017 (Network Rail, 2004a). 16 potential intervals were chosen, ranging between 1 to 16 years.

2. Opportunistic maintenance (applies to the whole structure)

a. Opportunistic maintenance can be enabled or disabled for an asset.

3. Intervention options (applies to individual elements)

a. Option 1: Intervene at good condition - repair as soon as any component is revealed to be in any degraded state (good or worse).

b. Option 2: Intervene at poor condition - carry out major repair when the component reaches the poor state.

c. Option 3: Intervene at very poor condition - only perform renewals when the component reaches the very poor state.

4. Servicing interval (applies to individual elements)

a. The servicing intervals considered in practice are set in (RT/CE/C/002, 2002). 16 possible servicing intervals were chosen, from 1 to 16 years.

5. Minor repair delay time (applies to individual elements)

a. In practice, Network Rail schedule interventions according to a prioritised work bank and subject to budget constraint. In additional, the design, preparation and management time also contribute to the delay time. It has been reported by bridge experts that the delay time depends on the type of work and can be up to 4 years (Halcrow, 2011). It was suggested that it is reasonable to assume that minor interventions can be delayed up to 2 years. Four possible values were chosen: $6,12,18$ and 24 months.

6. Major repair delay time (applies to individual elements)

a. It was also suggested by bridge experts that major intervention can also be delayed up to 2 years, thus four possible values were chosen: $6,12,24$ and 36 months.

7. Renewal delay time (applies to individual elements)

a. Eight possible delay times were chosen: $6,12,18,24$, $30,36,42$ and 48 months.

Table 7.1 summarises all the possible values which the variables can take. Note that the inspection intervals and opportunistic 
maintenance are set for the whole bridge structure, while other variables can be set for each bridge components individually.

\subsubsection{Variable encoding and decoding}

The GA often uses binary to represent the variable values, this process is called the variable encoding. Each variable is represented by a string of bits, a bit can take a value of 0 or 1 , depending on the range of possible values that a particular variable can take, an appropriate number of bits is required. The binary strings representing each variable are then listed contiguously to form an individual that represents a unique possible maintenance strategy. An example is given in Figure 7.2 of the encoding process for three variables. Variable 1 can take 16 values so 4 bits string is used to represents these $2^{4}=16$ options. Variable 2 can have 64 possible values so a string of 6 bits is used. Similarly, variable 3 uses a 3-bit string to represent 8 possible values. Thus, a string of total 13 bits long is used to represent an individual.

$$
\text { an individual }=\underbrace{4 \text { bits }}_{\text {var } 1} \underbrace{6 \text { bits }}_{\text {var } 2} \underbrace{3 \text { bits }}_{\text {var } 3}
$$

Figure 7.2: Example of variable coding and decoding process

\begin{tabular}{|l|l|c|c|c|}
\hline Variables & \multicolumn{1}{|c|}{$\begin{array}{c}\text { Value } \\
\text { range }\end{array}$} & Apply to & $\begin{array}{c}\text { Number of } \\
\text { options for } \\
\text { the selected } \\
\text { variable }\end{array}$ & $\begin{array}{c}\text { Number of bits } \\
\text { required to } \\
\text { represent } \\
\text { variable } \\
\text { selection }\end{array}$ \\
\hline $\begin{array}{l}\text { Inspection } \\
\text { period }\end{array}$ & $\begin{array}{l}1: 1: 16 \\
\text { years }\end{array}$ & Asset & 16 & 4 \\
\hline $\begin{array}{l}\text { Opportunistic } \\
\text { maintenance }\end{array}$ & $\begin{array}{l}0,1 \\
\text { (enabled, } \\
\text { disabled) }\end{array}$ & Asset & 2 & 1 \\
\hline $\begin{array}{l}\text { Intervention } \\
\text { options }\end{array}$ & $1,2,3$ & Component & 3 & 2 \\
\hline $\begin{array}{l}\text { Servicing } \\
\text { interval }\end{array}$ & $\begin{array}{l}1: 1: 16 \\
\text { years }\end{array}$ & Component & 16 & 2 \\
\hline $\begin{array}{l}\text { Minor repair } \\
\text { delay time }\end{array}$ & $\begin{array}{l}6,12,18,24 \\
\text { months }\end{array}$ & Component & 4 & 2 \\
\hline $\begin{array}{l}\text { Major repair } \\
\text { delay time }\end{array}$ & $\begin{array}{l}6,12,24,36 \\
\text { months }\end{array}$ & Component & 4 & 3 \\
\hline $\begin{array}{l}\text { Renewal delay } \\
\text { time }\end{array}$ & $\begin{array}{l}6: 6: 48 \\
\text { months }\end{array}$ & Component & 8 & 2 \\
\hline
\end{tabular}

Table 7.1: Model variables and their value range

For the range of variable values shown in Table 7.1, firstly, a 13bit string (Figure 7.3) is used to code all the variables that apply to a bridge component. Secondly, by combining these coded 
binary strings for each bridge component (Figure 7.4), a complete binary string is formed to represent a unique maintenance policy to an asset. For the bridge model that contains 8 major elements, a 109-bit string is required to code the variables. This also means that there are a total of about $2^{109}$ possible combinations of variables in the optimisation search space.

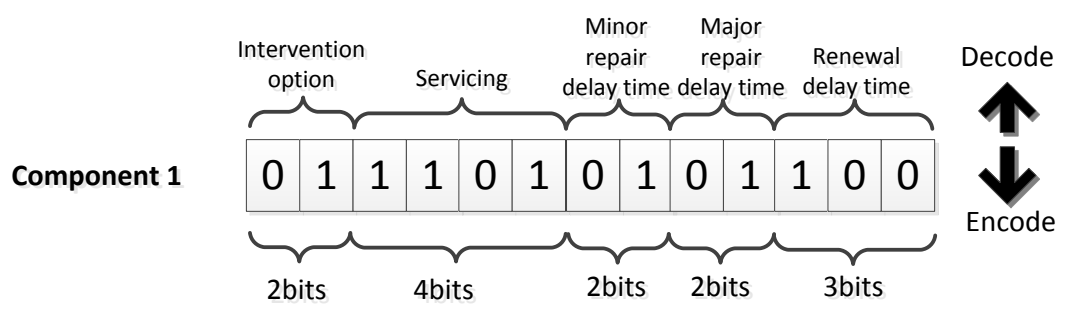

Figure 7.3: 13 bits string is used to code variables for a single bridge component

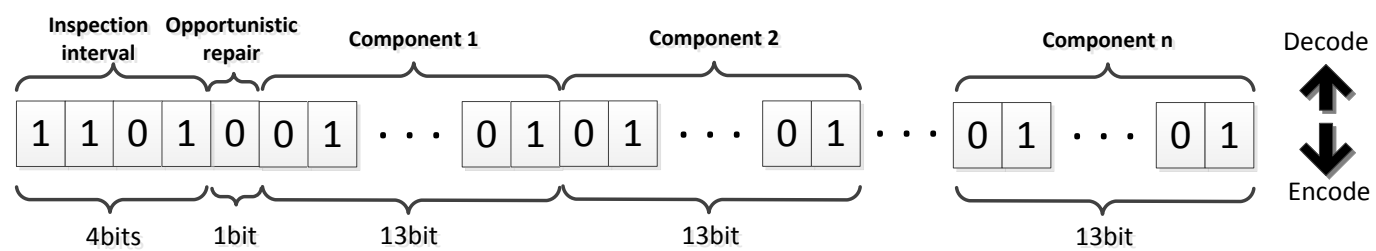

Figure 7.4: 109 bits string is used to code variables for a complete bridge asset contains 8 major elements

The binary string provides a mapping of each possible value of any variable. For example, a 4 bits string is used to represent the variable 'servicing interval', this binary string when decoded gives these possible values $0,1,2 \ldots 15$. However the actual values that servicing interval can take is $1,2,3 \ldots 16$ years, the coding/decoding then maps value 0 to represent a 1 year servicing interval, value 1 to represent 2 year, etc. This extra step of mapping completes the coding process and allows a binary string to represent any variable value. It is common that the number of options that any variable can take is not a convenient power of 2 . For example, there are 3 possible intervention options in the model and a 2-bit string is used to represent this variable, however this means that there are 4 possible values represented by the string. The mapping would be slightly different to adapt to this situation. A two bits string when decoded can take the values: $0,1,2,3$. The last two values 2 and 3 are then both used to map to one intervention option (option 3) as shown in Table 7.2 . 


\begin{tabular}{|c|c|c|c|}
\hline $\begin{array}{c}\text { Intervention } \\
\text { option }\end{array}$ & $\begin{array}{c}\text { Binary } \\
\text { representation }\end{array}$ & $\begin{array}{c}\text { Real } \\
\text { value }\end{array}$ & $\begin{array}{c}\text { Mapped to variable } \\
\text { value }\end{array}$ \\
\hline Option 1 & 00 & 0 & 1 \\
\hline Option 2 & 01 & 1 & 2 \\
\hline Option 3 & 10 & 2 & 3 \\
\hline Option 3 & 11 & 3 & 3 \\
\hline
\end{tabular}

Table 7.2: Coding of intervention option

\subsubsection{The population}

The GA starts with a group of individuals known as the population. The population is a matrix filled with ones and zeros (binary representation). The number of individuals in the population is the number of rows in the matrix and the number of bits is the number of columns. A population size of 20 was chosen in this optimisation exercise. The initial population was randomly generated which is a matrix of size $20 \times 109$.

It is recognised that the selection of the optimum optimisation parameters such as: the population size, selection function, selection rate, mutation rate, and number of elite individuals can be difficult as the changes in these parameter might produce different results or might even stop the GA to converge. In this research, these parameters are chosen based on "typical" values that are often accepted as default/safe values to be used in a GA optimisation exercise as found in the literature.

\subsubsection{Fitness function}

Each row of the matrix in the population is an individual in the solution search space, these bit-strings are decoded and passed to the objective function for evaluation. A fitness function would then determine the fittest individual by comparing the objective function values produced by each individual. For example, if we were to optimise the maintenance costs, the objective function is used to evaluate the maintenance cost for each individual. A fitness function then determines the fittest individual by ranking the costs produced by each individual. The individual which produces lowest cost in the population is the fittest.

\section{Pareto ranking}

It is relatively simple to rank the fitness of each individual in a single objective optimisation exercise. However for a multiobjective optimisation, a particular ranking technique is employed, which uses the Pareto dominance scheme (Fonseca 
and Fleming, 1993) for individual comparison. Figure 7.5 illustrates the objective ranking technique. It shows two objectives values ( $f 1$ - life cycle cost and f2 - average asset condition) for each individual in a population of candidate solutions. These are evaluated and plotted.

The candidate solution labelled " 4 " is dominated by four solutions within the rectangle of which it is a vertex. That is, four solutions exist which dominate it because they have better values of both objectives (criteria) in this optimisation problem.

The candidate solutions labelled "0" are said to be nondominated, as no other candidate solutions exist which improve on both of their criterion values.

Using this concept, an algorithm is written to rank the solutions, many solutions might have the same rank and a group of these solutions are called a front. After the ranking process, there might be several fronts, as illustrated, the first front (rank 0) is a population of five non-dominated individuals, the second front (rank 1) consists of one individuals dominated only by the first front and so on. In solving the optimisation problem, it is the nondominated solutions which are of interest which occur on the Pareto-front.

f2,

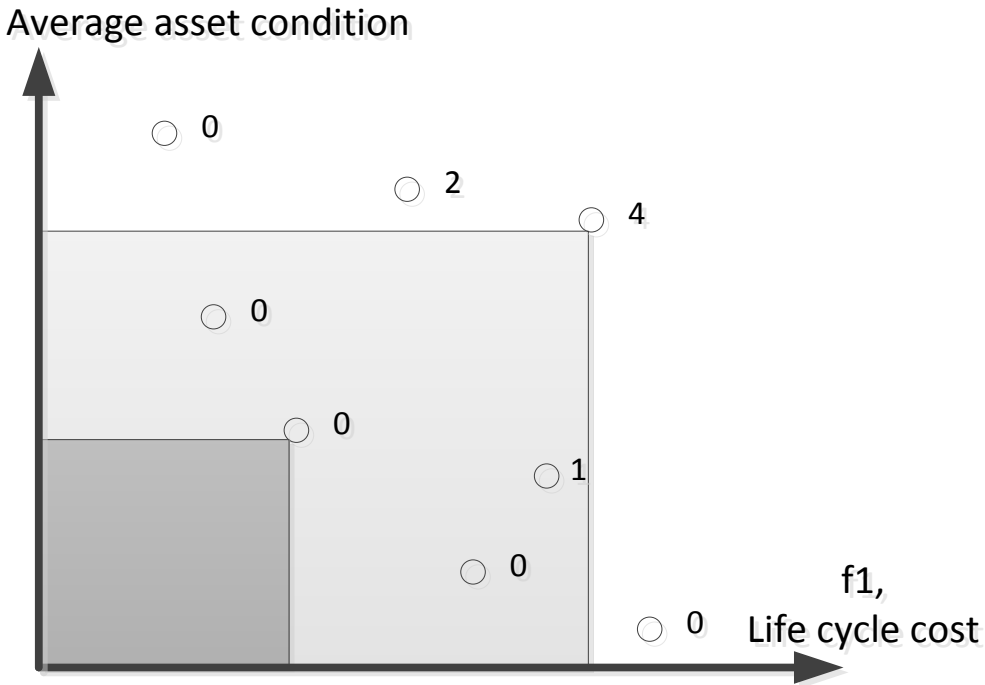

Figure 7.5: Pareto ranking technique 


\section{Selection rates}

A selection rate, $X_{\text {rate, }}$ is the fraction of the population that survive for the next step of mating. The rest are discarded to make room for the new offspring. The selection rate decides how many individuals to keep. If only a few are selected to the next generation, this will limit the available genes in the offspring. Keeping too many will allow a bad performer a chance to contribute bad genes to the next generation. The selection rate was set at 0.5 which means that when all individuals in the population of 20 are ranked, the 10 fittest individuals are kept giving 10 free spaces in the population to be filled in the next step.

\subsubsection{Paring (selection function)}

Pairing or mating of individuals will create offspring and this process is repeated until the free places in the population are filled with the new offspring. There are many ways to pair individuals, the most common is by random paring. Other methods can be employed such as weighted paring (where the chance of fittest individual being mated is higher than less-fit individual), tournament selection (randomly pick a small subset of individuals and the fittest of these will become a parent, this is best for larger population size and does not require sorting).

The technique was used in this research is rank pairing (Haupt and Haupt, 2004). In this approach, the individuals are selected randomly according to a rank probability calculated according to Equation (2.5).

$$
P_{n}=\frac{N-n+1}{\sum_{n=1}^{N} n}=\frac{11-n}{55}
$$

As an example, the 10 fittest individuals in a population are listed in Table 7.3. Their fitness values are calculated and they are ranked according to the Pareto-front. The rank probability for each individual can then be calculated based on its position from the top of the list. The last column shows the cumulative rank probability.

A random number between zero and one is then generated, the first individual with a cumulative probability that is greater than the random number is selected for paring. For instance, if the random number is $r=0.5555$, then since $0.4909<r<0.6182$, thus 
individual number 4 is selected. This process is repeated to identify the second individual to pair. There is a chance that an individual might be selected twice and would be paired with itself. If this happens, the process is repeated again until a different individual is selected. It is worth noting that the rank probabilities only have to be calculated once and would be the same in all generations.

\begin{tabular}{|c|c|c|c|c|c|c|}
\hline $\mathbf{n}$ & $\begin{array}{c}\text { Individuals } \\
(109 \text {-bit string) }\end{array}$ & $\begin{array}{c}\text { Fitness value 1 } \\
\text { (avg. cond.) }\end{array}$ & $\begin{array}{c}\text { Fitness } \\
\text { value 2 } \\
\text { (LCC } £ \text { ) }\end{array}$ & $\begin{array}{c}\text { Pareto- } \\
\text { front } \\
\text { ranking }\end{array}$ & $\boldsymbol{P}_{\boldsymbol{i}}$ & $\sum_{\boldsymbol{i = 1}}^{\boldsymbol{n}} \boldsymbol{P}_{\boldsymbol{i}}$ \\
\hline 1 & $010 \ldots 101$ & 2.023 & $1.95 \mathrm{E}+05$ & 0 & 0.1818 & 0.1818 \\
\hline 2 & $101 \ldots 100$ & 1.733 & $2.24 \mathrm{E}+05$ & 0 & 0.1636 & 0.3455 \\
\hline 3 & $110 \ldots 111$ & 1.540 & $2.27 \mathrm{E}+05$ & 0 & 0.1455 & 0.4909 \\
\hline 4 & $101 \ldots 110$ & 1.464 & $2.32 \mathrm{E}+05$ & 0 & 0.1273 & 0.6182 \\
\hline 5 & $101 \ldots 110$ & 1.580 & $2.31 \mathrm{E}+05$ & 1 & 0.1091 & 0.7273 \\
\hline 6 & $110 \ldots 011$ & 1.756 & $2.25 \mathrm{E}+05$ & 1 & 0.0909 & 0.8182 \\
\hline 7 & $111 \ldots 100$ & 1.552 & $2.30 \mathrm{E}+05$ & 1 & 0.0727 & 0.8909 \\
\hline 8 & $110 \ldots 011$ & 2.022 & $2.13 \mathrm{E}+05$ & 1 & 0.0545 & 0.9455 \\
\hline 9 & $110 \ldots 100$ & 2.276 & $2.16 \mathrm{E}+05$ & 2 & 0.0364 & 0.9818 \\
\hline 10 & $010 \ldots 111$ & 2.137 & $2.28 \mathrm{E}+05$ & 2 & 0.0182 & 1.0000 \\
\hline
\end{tabular}

Table 7.3: Rank probability of 10 fittest individuals

\subsubsection{Mating}

When two parents mate to produce offspring, the offspring are placed into the population. To do this, two crossover points are randomly selected between the first and last bits of the parents' strings, between these points, each part of the string from the parents is combined to make the complete string of the offspring. The mating process is illustrated in Figure 7.6.

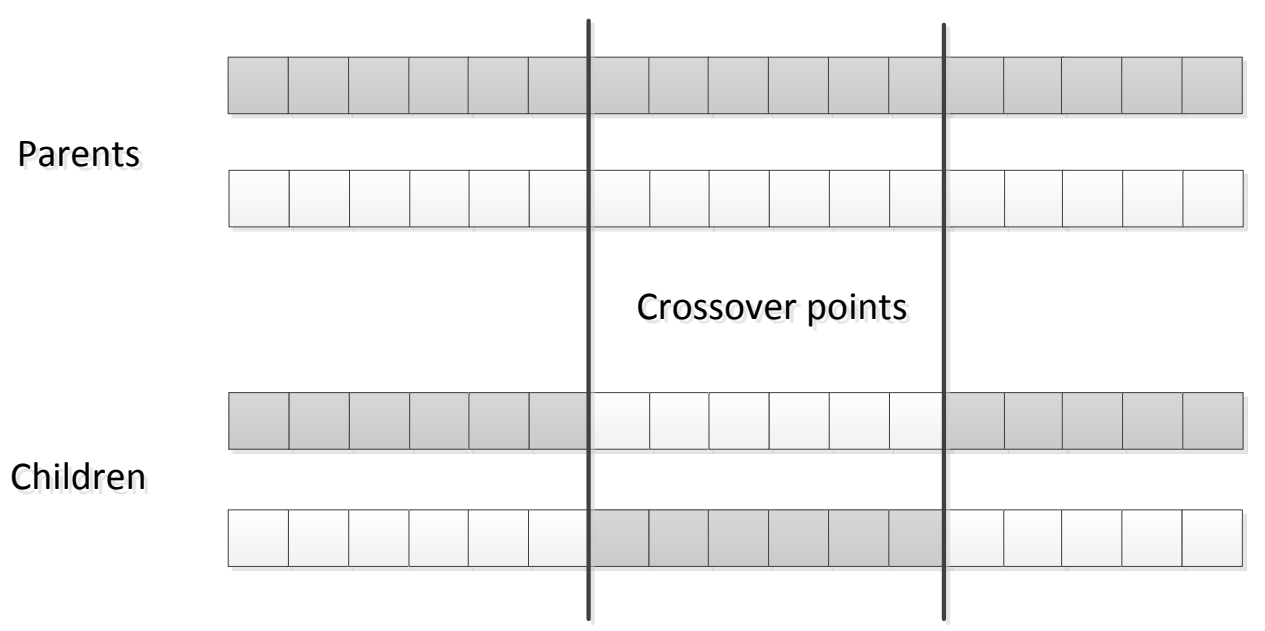

Figure 7.6: Two-point cross-over 


\subsubsection{Mutation}

Random mutations alter a certain percentage of the bits in the string. Mutation provides genetic diversity and allows the GA to explore a broader space and prevent it from converging too fast to a local optimal point before searching the entire space. A single point mutation changes a bit from value 0 to 1 or vice versa. $A$ mutation rate can be set which indicates the number of bits will be mutated in the population. In this optimisation, each individual is a 109 -bits string in a population size of 20 . This would create a population of $20 \times 109=2180$ bits. A mutation rate of 0.005 was used, this means that $0.5 \%$ of the bits in the entire 2180 bits population will be randomly mutated.

However, the problem with mutation is that it also mutates the best solutions which can prevent the GA converging to the best solutions. To prevent the mutation on the best solution, elitism is introduced where a selection of fittest individuals are called elites. An algorithm was developed so there are no mutations on these elites and the first 5 fittest individuals in the population were set as elite individuals.

\subsubsection{Next generation and convergence}

After the mutation, the objective functions are evaluated for the offspring generated by the pairing, mating and mutation processes. The process of pairing, mating and mutation is then repeated again for the next population. This is repeated for a number of generations which depends on whether the process has converged. The convergence is considered when the values of the objective function evaluations do not change from generation to generation. In this work, the convergence is considered to be achieved when all the individuals are in the top rank (rank 0 - the first Pareto front). 


\subsection{Results and discussions}

\subsubsection{Optimum policies}

Figure 7.7 shows snapshots of the population performance at each generation. At the generation number 1 , the optimisation starts with a population of 20 randomly guessed maintenance policies. The objective function values of this population are evaluated and plotted. At generation number 2, the plot shows that the population is slightly shifted towards the origin of the axes indicating that the evolved population is now fitter than the previous population. The process continues and converges by the $30^{\text {th }}$ generation since all the solutions are now in the first Pareto front.

The final generation contains a set of 20 optimum maintenance policies. The details of the 20 maintenance policies appearing in the optimised Pareto front are shown in Table 7.4. The trade-off between these policies is clearly demonstrated in the figures as the maintenance cost increases when the average asset condition improves (decreases). Interestingly, it has been observed throughout the optimisation process that there is no maintenance policy that would result in the asset average condition being in the poor or lower condition. The model predicts that, for as long as there are interventions being applied, the average asset condition would never be in the very poor condition. 

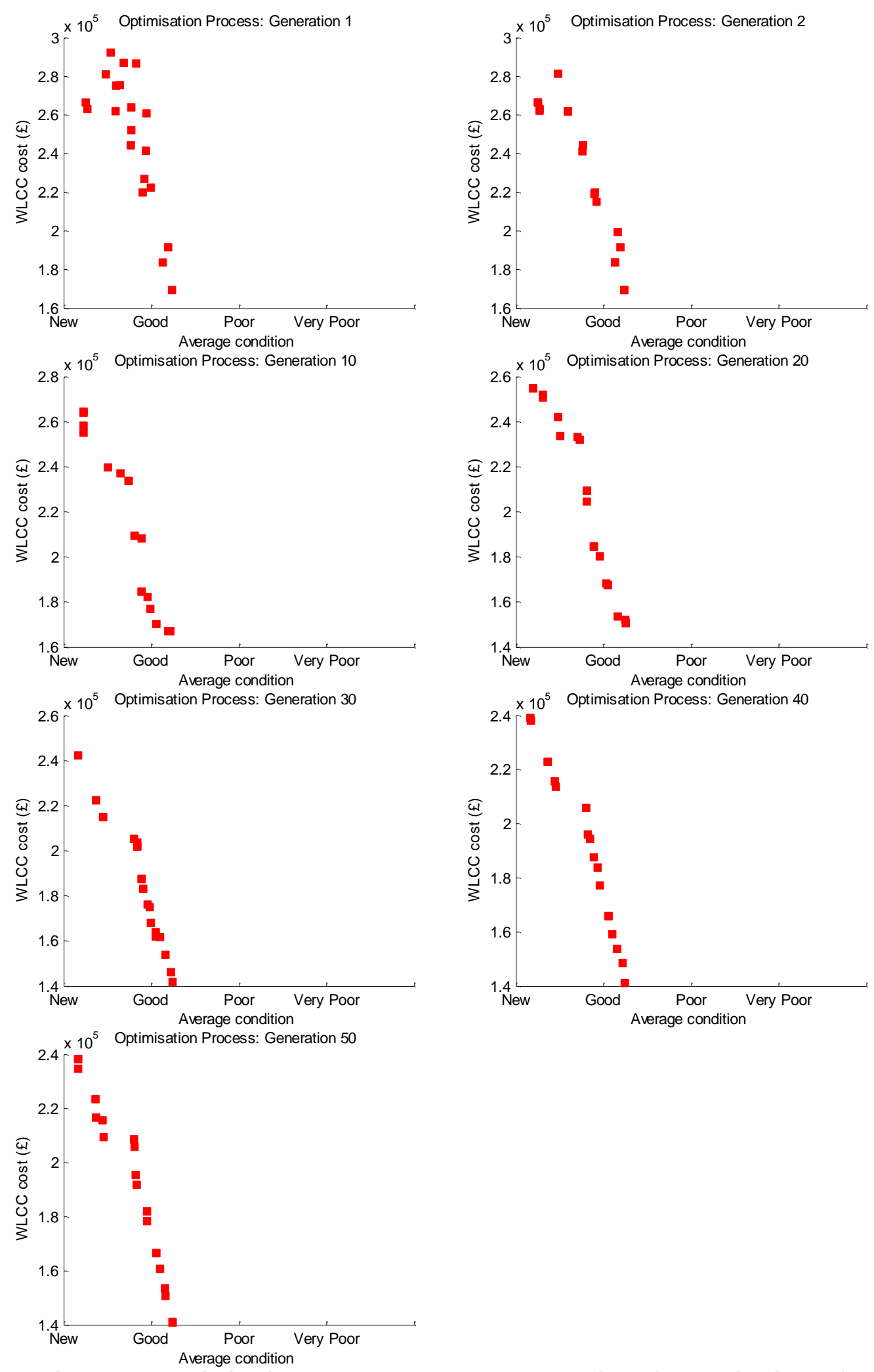

Figure 7.7: Optimisation process shows the evaluation of the objectives of the population at each generation. Convergence happens at around the $30^{\text {th }}$ generation. 


\begin{tabular}{|c|c|c|c|c|c|c|c|c|c|c|c|c|c|c|c|c|c|c|c|c|c|}
\hline & Policy ID & 1 & 2 & 3 & 4 & 5 & 6 & 7 & 8 & 9 & 10 & 11 & 12 & 13 & 14 & 15 & 16 & 17 & 18 & 19 & 20 \\
\hline \multicolumn{2}{|c|}{ Inspection period } & 3 & 3 & 16 & 3 & 3 & 3 & 3 & 3 & 6 & 6 & 16 & 11 & 16 & 3 & 11 & 3 & 13 & 3 & 3 & 16 \\
\hline \multicolumn{2}{|c|}{ Opportunistic maintenance } & 1 & 1 & 0 & 0 & 0 & 1 & 0 & 0 & 0 & 0 & 0 & 0 & 0 & 0 & 0 & 0 & 0 & 0 & 0 & 0 \\
\hline \multirow{5}{*}{ DCK } & Intervention opt. & 1 & 1 & 3 & 1 & 3 & 1 & 1 & 3 & 3 & 3 & 3 & 3 & 3 & 1 & 3 & 3 & 3 & 3 & 1 & 3 \\
\hline & Servicing interval & 1 & 1 & 3 & 3 & 10 & 3 & 4 & 3 & 9 & 9 & 3 & 3 & 3 & 3 & 3 & 10 & 1 & 9 & 4 & 3 \\
\hline & Minor repair delay & 18 & 18 & 18 & 18 & 18 & 18 & 18 & 18 & 18 & 18 & 18 & 18 & 18 & 18 & 18 & 18 & 18 & 18 & 18 & 18 \\
\hline & Major repair delay & 24 & 24 & 24 & 24 & 24 & 24 & 24 & 24 & 24 & 24 & 24 & 24 & 24 & 24 & 24 & 24 & 24 & 24 & 24 & 24 \\
\hline & Renewal delay & 12 & 12 & 30 & 30 & 30 & 18 & 18 & 30 & 24 & 24 & 42 & 24 & 24 & 36 & 24 & 42 & 30 & 42 & 18 & 30 \\
\hline \multirow{5}{*}{ MGE1 } & Intervention opt. & 2 & 2 & 3 & 3 & 2 & 2 & 3 & 2 & 3 & 3 & 3 & 3 & 3 & 3 & 3 & 2 & 3 & 2 & 3 & 3 \\
\hline & Servicing interval & 4 & 4 & 3 & 3 & 4 & 4 & 2 & 2 & 5 & 5 & 3 & 4 & 4 & 3 & 4 & 4 & 4 & 3 & 2 & 2 \\
\hline & Minor repair delay & 18 & 18 & 18 & 18 & 18 & 18 & 18 & 18 & 18 & 18 & 18 & 18 & 18 & 18 & 18 & 18 & 18 & 18 & 18 & 18 \\
\hline & Major repair delay & 12 & 12 & 36 & 24 & 12 & 12 & 12 & 24 & 12 & 12 & 36 & 36 & 36 & 24 & 36 & 12 & 36 & 36 & 12 & 36 \\
\hline & Renewal delay & 6 & 6 & 36 & 42 & 12 & 30 & 36 & 36 & 12 & 12 & 36 & 24 & 24 & 42 & 24 & 24 & 42 & 12 & 36 & 36 \\
\hline \multirow{5}{*}{ MGI } & Intervention opt. & 1 & 1 & 1 & 1 & 1 & 1 & 1 & 1 & 1 & 1 & 1 & 1 & 1 & 1 & 1 & 1 & 1 & 1 & 1 & 1 \\
\hline & Servicing interval & 2 & 2 & 5 & 7 & 3 & 1 & 5 & 3 & 2 & 2 & 5 & 3 & 3 & 7 & 3 & 3 & 4 & 3 & 4 & 5 \\
\hline & Minor repair delay & 18 & 18 & 24 & 24 & 24 & 24 & 18 & 24 & 24 & 18 & 24 & 24 & 24 & 24 & 24 & 24 & 24 & 24 & 18 & 24 \\
\hline & Major repair delay & 12 & 12 & 24 & 24 & 12 & 12 & 12 & 24 & 24 & 24 & 24 & 12 & 12 & 24 & 12 & 12 & 24 & 24 & 12 & 24 \\
\hline & Renewal delay & 30 & 30 & 36 & 30 & 24 & 30 & 30 & 36 & 30 & 30 & 36 & 24 & 24 & 30 & 24 & 24 & 30 & 30 & 30 & 36 \\
\hline \multirow{5}{*}{ MGE2 } & Intervention opt. & 2 & 2 & 3 & 3 & 2 & 2 & 3 & 2 & 3 & 3 & 3 & 3 & 3 & 3 & 3 & 2 & 3 & 2 & 3 & 3 \\
\hline & Servicing interval & 4 & 4 & 3 & 3 & 4 & 4 & 2 & 2 & 5 & 5 & 3 & 4 & 4 & 3 & 4 & 4 & 4 & 3 & 2 & 2 \\
\hline & Minor repair delay & 18 & 18 & 18 & 18 & 18 & 18 & 18 & 18 & 18 & 18 & 18 & 18 & 18 & 18 & 18 & 18 & 18 & 18 & 18 & 18 \\
\hline & Major repair delay & 12 & 12 & 36 & 24 & 12 & 12 & 12 & 24 & 12 & 12 & 36 & 36 & 36 & 24 & 36 & 12 & 36 & 36 & 12 & 36 \\
\hline & Renewal delay & 6 & 6 & 36 & 42 & 12 & 30 & 36 & 36 & 12 & 12 & 36 & 24 & 24 & 42 & 24 & 24 & 42 & 12 & 36 & 36 \\
\hline \multirow{2}{*}{ BGL1 } & Intervention opt. & 1 & 1 & 3 & 3 & 2 & 1 & 1 & 3 & 1 & 1 & 1 & 1 & 1 & 1 & 1 & 2 & 1 & 2 & 3 & 3 \\
\hline & Servicing interval & 1 & 1 & 4 & 3 & 1 & 2 & 1 & 2 & 2 & 1 & 4 & 1 & 1 & 3 & 1 & 3 & 2 & 1 & 2 & 4 \\
\hline
\end{tabular}




\begin{tabular}{|c|c|c|c|c|c|c|c|c|c|c|c|c|c|c|c|c|c|c|c|c|c|}
\hline & Minor repair delay & 18 & 18 & 18 & 18 & 24 & 24 & 18 & 18 & 24 & 24 & 18 & 18 & 18 & 18 & 18 & 24 & 18 & 24 & 18 & 18 \\
\hline & Major repair delay & 24 & 24 & 24 & 24 & 12 & 24 & 24 & 24 & 12 & 12 & 24 & 12 & 12 & 24 & 12 & 12 & 24 & 12 & 24 & 24 \\
\hline & Renewal delay & 30 & 30 & 30 & 36 & 18 & 30 & 36 & 30 & 36 & 24 & 30 & 18 & 18 & 36 & 18 & 24 & 18 & 24 & 36 & 30 \\
\hline \multirow{5}{*}{ BGL2 } & Intervention opt. & 1 & 1 & 3 & 3 & 2 & 1 & 1 & 3 & 1 & 1 & 1 & 1 & 1 & 1 & 1 & 2 & 1 & 2 & 3 & 3 \\
\hline & Servicing interval & 1 & 1 & 4 & 3 & 1 & 2 & 1 & 2 & 2 & 1 & 4 & 1 & 1 & 3 & 1 & 3 & 2 & 1 & 2 & 4 \\
\hline & Minor repair delay & 18 & 18 & 18 & 18 & 24 & 24 & 18 & 18 & 24 & 24 & 18 & 18 & 18 & 18 & 18 & 24 & 18 & 24 & 18 & 18 \\
\hline & Major repair delay & 24 & 24 & 24 & 24 & 12 & 24 & 24 & 24 & 12 & 12 & 24 & 12 & 12 & 24 & 12 & 12 & 24 & 12 & 24 & 24 \\
\hline & Renewal delay & 30 & 30 & 30 & 36 & 18 & 30 & 36 & 30 & 36 & 24 & 30 & 18 & 18 & 36 & 18 & 24 & 18 & 24 & 36 & 30 \\
\hline \multirow{5}{*}{ ABT1 } & Intervention opt. & 3 & 3 & 3 & 2 & 2 & 3 & 3 & 3 & 1 & 1 & 3 & 3 & 3 & 2 & 3 & 2 & 3 & 2 & 3 & 3 \\
\hline & Servicing interval & 8 & 8 & 9 & 10 & 8 & 10 & 11 & 9 & 12 & 12 & 9 & 8 & 8 & 10 & 8 & 8 & 9 & 8 & 11 & 9 \\
\hline & Minor repair delay & 18 & 18 & 24 & 24 & 24 & 24 & 18 & 24 & 24 & 24 & 24 & 24 & 24 & 24 & 24 & 24 & 24 & 24 & 18 & 24 \\
\hline & Major repair delay & 12 & 12 & 12 & 12 & 12 & 12 & 12 & 12 & 12 & 12 & 12 & 12 & 12 & 12 & 12 & 12 & 12 & 12 & 12 & 12 \\
\hline & Renewal delay & 18 & 18 & 42 & 18 & 48 & 12 & 18 & 42 & 42 & 42 & 12 & 48 & 48 & 18 & 48 & 48 & 48 & 48 & 18 & 42 \\
\hline \multirow{5}{*}{ ABT2 } & Intervention opt. & 3 & 3 & 3 & 2 & 2 & 3 & 3 & 3 & 1 & 1 & 3 & 3 & 3 & 2 & 3 & 2 & 3 & 2 & 3 & 3 \\
\hline & Servicing interval & 8 & 8 & 9 & 10 & 8 & 10 & 11 & 9 & 12 & 12 & 9 & 8 & 8 & 10 & 8 & 8 & 9 & 8 & 11 & 9 \\
\hline & Minor repair delay & 18 & 18 & 24 & 24 & 24 & 24 & 18 & 24 & 24 & 24 & 24 & 24 & 24 & 24 & 24 & 24 & 24 & 24 & 18 & 24 \\
\hline & Major repair delay & 12 & 12 & 12 & 12 & 12 & 12 & 12 & 12 & 12 & 12 & 12 & 12 & 12 & 12 & 12 & 12 & 12 & 12 & 12 & 12 \\
\hline & Renewal delay & 18 & 18 & 42 & 18 & 48 & 12 & 18 & 42 & 42 & 42 & 12 & 48 & 48 & 18 & 48 & 48 & 48 & 48 & 18 & 42 \\
\hline \multicolumn{2}{|c|}{ Average condition } & 1.162 & 1.162 & 2.241 & 1.453 & 1.802 & 1.166 & 1.361 & 1.832 & 1.951 & 1.950 & 2.162 & 2.054 & 2.156 & 1.370 & 2.054 & 1.809 & 2.100 & 1.818 & 1.446 & 2.240 \\
\hline \multicolumn{2}{|c|}{ LCC over 60 years (kf) } & 238.2 & 238.2 & 141.0 & 209.4 & 208.6 & 234.8 & 223.5 & 191.8 & 178.3 & 182.0 & 150.6 & 166.5 & 153.5 & 216.7 & 166.5 & 205.8 & 160.7 & 195.4 & 215.7 & 141.1 \\
\hline
\end{tabular}

Table 7.4: 20 optimum maintenance policies after the optimisation of 50 generations. Unit- inspection and servicing interval: years; delay repair: months; opportunistic repair: 1-enabled, 0-disabled. 
7.3.2 Comparison to industry maintenance policy

\begin{tabular}{|c|l|}
\hline Policy & \multicolumn{1}{c|}{ Description } \\
\hline Standard & $\begin{array}{l}\text { This strategy is to intervene on bridges at a high level of tolerable } \\
\text { risk and to maintain the structures within a good condition state. }\end{array}$ \\
\hline Minimum & $\begin{array}{l}\text { This strategy adopts a lower level of tolerable risk and accepts } \\
\text { elements within the bridge may be in good or poor condition. }\end{array}$ \\
\hline Managed & $\begin{array}{l}\text { This strategy was developed to maintain current condition states } \\
\text { and assumes the lowest level of tolerable risk. }\end{array}$ \\
\hline
\end{tabular}

Table 7.5: Maintenance policies employed by Network Rail (Halcrow, 2011)

Maintenance policies that are currently employed by Network Rail on managing railway bridges are given in Table 7.5. Network Rail has carried out case studies on a group of assets to determine the optimum policies that would produce the lowest LCC. Thus for any asset type with a known initial condition, the optimum maintenance policy can be identified. For the selected example metal underbridge used in this research, since the initial condition of the asset is just below the poor condition, the maintenance policy that is believed by Network Rail to produce the lowest WLCC is the 'standard' maintenance policy. This industry policy can be implemented by running the bridge model with intervention strategy 1 -which is to intervene when the asset reaches the good condition. Table 7.6 shows the performances of the industry maintenance policy and a comparable policy that has been chosen from the set of optimised policies. The comparable policy is selected to show improved performance both in terms of the average asset condition and the expected WLCC. For this reason policy number 6 is selected for the comparison. The improved performance is achieved by the model's ability to consider the initial condition of each element and set the intervention accordingly. This can be seen in the table where different intervention options are set for the external girders and the abutments.

\begin{tabular}{|c|c|c|c|c|c|c|c|c|c|c|c|}
\hline \multirow{2}{*}{$\begin{array}{l}\text { Policy } \\
\text { ID }\end{array}$} & \multirow{2}{*}{$\begin{array}{c}\text { Opp. } \\
\text { maint. }\end{array}$} & \multicolumn{8}{|c|}{ Specific intervention to each bridge component } & \multirow{2}{*}{$\begin{array}{l}\text { Avg. } \\
\text { cond. }\end{array}$} & \multirow{2}{*}{$\begin{array}{l}\text { LCC } \\
(f)\end{array}$} \\
\hline & & DCK & MGE1 & MGI & MGE2 & BGL1 & BGL2 & ABT1 & ABT2 & & \\
\hline 6 & enable & $\begin{array}{c}\text { repair } \\
\text { asap }\end{array}$ & $\begin{array}{l}\text { only } \\
\text { major } \\
\text { repair }\end{array}$ & $\begin{array}{c}\text { repair } \\
\text { asap }\end{array}$ & $\begin{array}{l}\text { only } \\
\text { major } \\
\text { repair }\end{array}$ & $\begin{array}{c}\text { repair } \\
\text { asap }\end{array}$ & $\begin{array}{c}\text { repair } \\
\text { asap }\end{array}$ & $\begin{array}{l}\text { only } \\
\text { replace }\end{array}$ & $\begin{array}{l}\text { only } \\
\text { replace }\end{array}$ & 1.166 & 234762 \\
\hline Industry & disable & $\begin{array}{l}\text { repair } \\
\text { asap }\end{array}$ & $\begin{array}{c}\text { repair } \\
\text { asap }\end{array}$ & $\begin{array}{c}\text { repair } \\
\text { asap }\end{array}$ & $\begin{array}{c}\text { repair } \\
\text { asap }\end{array}$ & $\begin{array}{c}\text { repair } \\
\text { asap }\end{array}$ & $\begin{array}{c}\text { repair } \\
\text { asap }\end{array}$ & $\begin{array}{c}\text { repair } \\
\text { asap }\end{array}$ & $\begin{array}{c}\text { repair } \\
\text { asap }\end{array}$ & 1.278 & 235366 \\
\hline
\end{tabular}

Table 7.6: Comparison of performance between industry maintenance policy and selected optimised maintenance policy 


\subsection{Petri-net bridge model optimisation}

In this section, the optimisation of the Petri-Net bridge model is discussed. The optimisation of the PN model is a hybrid optimisation, which utilises the optimisation results from the Markov bridge models as the initial guesses for the optimisation of the PN bridge model. This results in a faster convergence to the optimum solution hence a shorter processing time. The rest of the MOGA optimisation procedure is very similar to the optimisation of the Markov bridge model as explained in the previous section. For instance, the objectives, the population size, fitness function, selection function, cross-over rate and mutation rate are the same. This section focuses only on the differences in the optimisation procedure between two models before discussing the results.

\subsubsection{Objective functions}

\section{Average condition of an asset}

The average condition of an asset is, again, determined by translating the probabilities of being in each condition states into a single value ranging between 1 and 4 . Therefore, the objective of maximising the average asset condition is achieved by minimising Equation (7.1) as previously discussed in section 7.2.1.

\section{Life Cycle Cost}

With different maintenance policy, the mean number of interventions, over the whole prediction period $\mathrm{T}$, on a single bridge element can be estimated using the model, thus the expected maintenance cost can be directly deduced. The objective of minimising the LCC of an asset is achieved by:

$$
\min _{0 \leq t \leq T}\left[\left[\sum_{i=1}^{N} n_{1}^{i} \times C_{1}^{i}+n_{2}^{i} \times C_{2}^{i}+n_{3}^{i} \times C_{3}^{i}+n_{4}^{i} \times C_{4}^{i}\right]+N_{S} C_{S}+N_{I} C_{I}\right]
$$

where $i \geq 1, i \in N$

$\mathrm{N}$ is the number of bridge component

$n_{1}^{i}$ is the expected number of minor repair on bridge component $i$

$n_{2}^{i}$ is the expected number of major repair on bridge component $i$

$n_{3}^{i}$ is the expected number of renewal on bridge component $i$

$n_{4}^{i}$ is the expected number of servicing on bridge component $i$

$C_{1}^{i}$ is the average cost of minor repair for bridge component $i$

$C_{2}^{i}$ is the average cost of major repair for bridge component $i$

$C_{3}^{i}$ is the average cost of renewal for bridge component $i$

$C_{4}^{i}$ is the average cost of servicing for bridge component $i$

$C_{I}, C_{S}, N_{I}, N_{S}$ are the avg. costs and number of inspection and servicing 


\subsubsection{Variables}

Table 7.7 shows the PN model variables and their value ranges. The PN model considers 4 intervention options for a single element, these options were discussed in Chapter 5 - 5.5.5. The model also allows the maintenance schedule of an asset to be varied in this optimisation exercise. The maintenance schedule is often set by the authority considering the maintenance of different parts on the railway network. Four maintenance schedules are possible, they are 4, 2, 1 maintenance blocks per year and one maintenance block every 2 years. The rest of the variables are similar to the Markov model. A 113-bit binary string is then needed to code all of the possible variable combinations for the whole bridge structure.

\begin{tabular}{|c|c|c|c|c|c|}
\hline Variables & $\begin{array}{l}\text { Apply } \\
\text { to }\end{array}$ & $\begin{array}{l}\text { Value } \\
\text { range }\end{array}$ & Apply to & $\begin{array}{l}\text { Number of } \\
\text { options } \\
\text { for the } \\
\text { selected } \\
\text { variable }\end{array}$ & $\begin{array}{l}\text { Number of } \\
\text { bits required } \\
\text { to represent } \\
\text { variable } \\
\text { selection }\end{array}$ \\
\hline $\begin{array}{c}\text { Inspection } \\
\text { period }\end{array}$ & Asset & $\begin{array}{c}1: 1: 16 \\
\text { years }\end{array}$ & Asset & 16 & 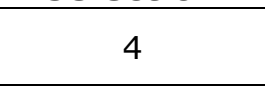 \\
\hline $\begin{array}{l}\text { Opportunistic } \\
\text { maintenance }\end{array}$ & Asset & $\begin{array}{c}0,1 \\
\text { (enabled, } \\
\text { disabled) }\end{array}$ & Asset & 2 & 1 \\
\hline $\begin{array}{l}\text { Maintenance } \\
\text { schedule }\end{array}$ & Element & $\begin{array}{l}4,2,1,0.5 \\
\text { time a year }\end{array}$ & Asset & 4 & 2 \\
\hline $\begin{array}{c}\text { Intervention } \\
\text { options }\end{array}$ & Element & $1,2,3,4$ & Component & 4 & 2 \\
\hline $\begin{array}{c}\text { Servicing } \\
\text { interval } \\
\text { (painting } \\
\text { interval for } \\
\text { metal element) }\end{array}$ & Element & $\begin{array}{l}1: 1: 16 \\
\text { years }\end{array}$ & Component & 16 & 4 \\
\hline $\begin{array}{c}\text { Minor repair } \\
\text { delay time }\end{array}$ & Element & $\begin{array}{c}6,12,18,24 \\
\text { months }\end{array}$ & Component & 4 & 2 \\
\hline $\begin{array}{l}\text { Major repair } \\
\text { delay time }\end{array}$ & Element & $\begin{array}{c}6,12,24,36 \\
\text { months }\end{array}$ & Component & 4 & 2 \\
\hline $\begin{array}{c}\text { Renewal delay } \\
\text { time }\end{array}$ & Element & $\begin{array}{l}6: 6: 48 \\
\text { months }\end{array}$ & Component & 8 & 3 \\
\hline
\end{tabular}

Table 7.7: PN model variables, value range and number of bits required

\subsubsection{Hybrid optimisation}

Generally at the start of the optimisation, the initial population is randomly generated. However if the initial population contains best guesses that are close to the optimum value, this increases the chance of finding the optimum value in a shorter time. This technique is applied in this section. Optimum maintenance policies, obtained after optimising the Markov bridge model, are 
used as starting points (initial population) in the optimisation of the PN model. This technique will be referred as the hybrid optimisation since the optimisation of the PN bridge model actually involves the optimisation of the Markov bridge model as well. The hybrid optimisation is possible in this case because:

- firstly, both the Markov and the PN model analyse the same asset,

- secondly, the two models share similar optimisation variables (e.g. intervention options, inspection period, servicing interval, repair delay time). Since the PN model is effectively a more detailed bridge model compared with the Markov model, it allows a wider range of maintenance policies to be applied,

- thirdly, the time it takes to run and optimise the Markov model is significantly faster than the PN model.

\section{Initial population}

Where possible, each optimisation variable in the PN model is directly mapped from the variable in the Markov model. For example, for a single bridge element, there are three intervention options in the Markov bridge model, they are equivalent to the $1^{\text {st }}, 2^{\text {nd }}$, and $4^{\text {th }}$ intervention option in the PN model. Similarly, the mapping can be done for other optimisation variables, except for the maintenance schedule variable as they were not included in the Markov model. Hence, random values within the given value range (Table 7.7 ) are generated for these variables.

Figure 7.8 illustrates a population which contains the bestguessed individuals and the randomly generated individuals. The green squares represent an initial population of randomly generated individuals. The red squares represent a population of 20 best-guessed individuals that are based on the maintenance policies obtained after optimising the Markov model. It can be clearly seen that, the guessed-individuals form a group that is closer to the origin than the randomly generated individuals. This means that, the hybrid optimisation process starts with a population that contains fitter individuals. 


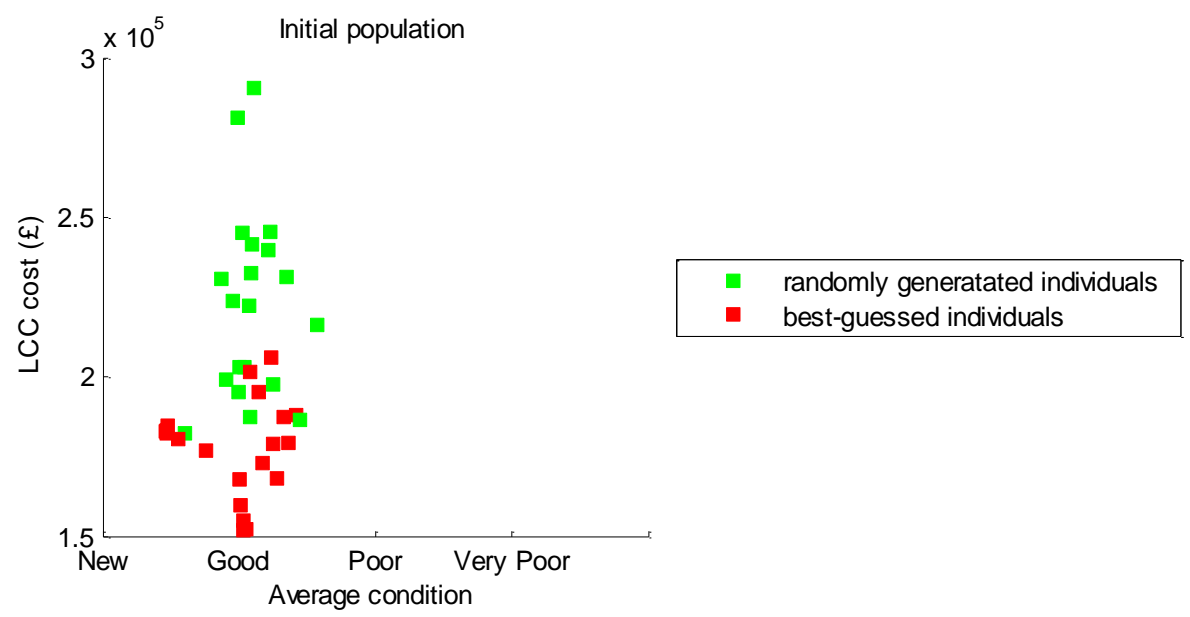

Figure 7.8: Initial population contains 20 best-guessed and 20 random individuals 


\subsection{Results and discussions}

\subsubsection{Optimum policies}
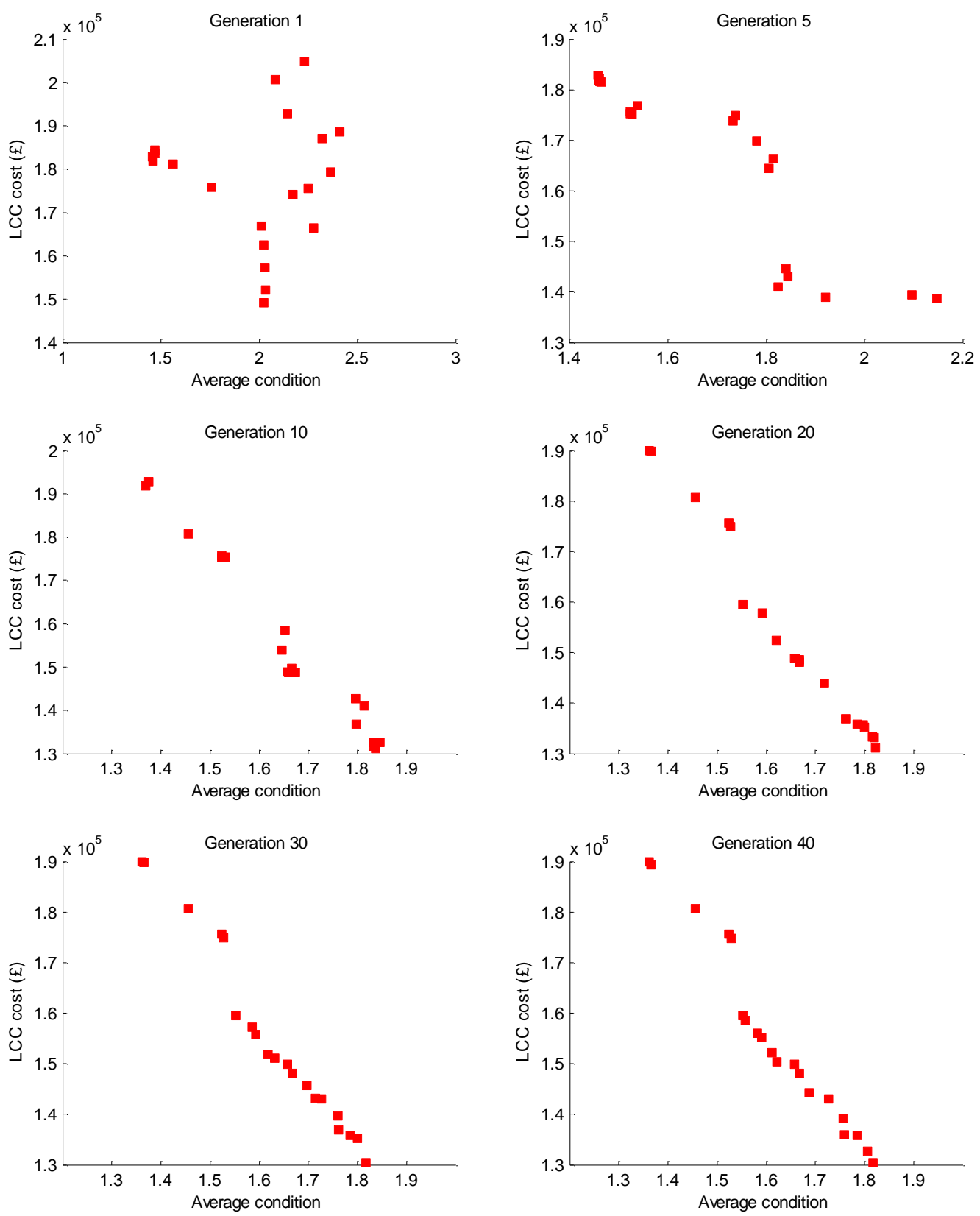

Figure 7.9: Optimisation process

Figure 7.9 plots the objective function values of each member of the population for each optimisation generation. The plot shows the gradual process of increasing the fitness of the individuals. Convergence was observed at around the $20^{\text {th }}$ generation. The final 20 optimum maintenance policies obtained, along with their performance in terms of average asset condition and WLCC, are show in Table 7.8. The details of the specific intervention option for each of the bridge elements are also given in the table. 
The first 6 maintenance policies have chosen the option to intervene as soon as any component degrades into the state where repair is possible. This reflects the intention to maximise the asset average condition by carrying out repair as soon as possible. These policies are expected to keep the asset condition very close to the 'as new' condition, although the associated LCC would be higher than other policies.

\begin{tabular}{|c|c|c|c|c|}
\hline Policy ID & Intervention option & Component & $\begin{array}{l}\text { Average } \\
\text { condition }\end{array}$ & $\begin{array}{l}\text { LCC over } \\
60 \text { years }\end{array}$ \\
\hline 1 & Repair as soon as possible & all components & 1.36 & 139493 \\
\hline 2 & Repair as soon as possible & all components & 1.43 & 136278 \\
\hline 3 & Repair as soon as possible & all components & 1.46 & 131968 \\
\hline 4 & Repair as soon as possible & all components & 1.46 & 130597 \\
\hline 5 & Repair as soon as possible & all components & 1.52 & 128700 \\
\hline 6 & Repair as soon as possible & all components & 1.53 & 125478 \\
\hline 7 & Only carry out minor repair and replacement for:* & MGI ABT1 & 1.55 & 110505 \\
\hline 8 & Only carry out minor repair and replacement for:* & MGI ABT1 & 1.56 & 109891 \\
\hline 9 & Only carry out replacement for: * & MGI ABT1 & 1.58 & 108720 \\
\hline 10 & Only carry out replacement for: * & MGI ABT1 & 1.59 & 108640 \\
\hline 11 & Only carry out replacement for: ${ }^{*}$ & MGI ABT1 & 1.59 & 105941 \\
\hline 12 & Only carry out replacement for: * & MGI ABT1 ABT2 & 1.61 & 101803 \\
\hline 13 & Only carry out replacement for: * & MGI ABT1 ABT2 & 1.62 & 101428 \\
\hline 14 & Only carry out minor repair and replacement for:* & MGI ABT1 ABT2 & 1.64 & 101087 \\
\hline 15 & Only carry out replacement for: ${ }^{*}$ & MGI ABT1 & 1.66 & 99231 \\
\hline 16 & Only carry out replacement for: * & MGI ABT1 & 1.67 & 97473 \\
\hline 17 & Only carry out replacement for: ${ }^{*}$ & MGI ABT1 ABT2 & 1.68 & 94092 \\
\hline 18 & Only carry out replacement for: $*$ & MGI ABT1 ABT2 & 1.69 & 92791 \\
\hline 19 & Only carry out replacement for: ${ }^{*}$ & MGI ABT1 ABT2 & 1.73 & 88777 \\
\hline 20 & Only carry out replacement for: * & MGI ABT1 ABT2 & 1.74 & 88229 \\
\hline
\end{tabular}

Table 7.8: 20 optimum maintenance policies and their performances. Also shown is the intervention option on each component. $\left({ }^{*}\right)$ indicates the specific intervention options for the listed elements, for other elements, they are repaired as soon as possible.

For other policies, whilst the intervention option is still set to intervene as soon as possible for the unspecified bridge elements, different intervention options were selected for the bridge internal main girder (MGI) and the abutments ( $A B T 1$ and $A B T 2$ ). Policies 7, 8 and 14 carry out only minor repair and replacement for the MGI. Recalling that the initial condition of the MGI is poor, this intervention option effectively allows the girder to deteriorate to a very poor state for a complete replacement. After a replacement, when the girder condition is restored to an 'as new' condition, the 
intervention is set to intervene as soon as possible. Therefore, skipping the major repair on the MGI and choosing only to replace, the optimisation shows that this strategy is an optimum way of lowering the maintenance cost while maximising the average condition over its lifetime. A similar strategy can be observed for the abutments. The abutments' initial conditions are 'as new', combining this with the slow rate of degradation, it is unlikely for the abutment to reach the poor condition in the simulated life time of 60 years. Therefore it is possible to allow the abutments to deteriorate without intervention and focus the attention and resources on the other components. The optimised policies 9-20 illustrate this strategy where the intervention option is set to carry out only replacement for the abutments. In general, it can be seen that for the selected asset, it is optimum to intervene as soon as possible for all the components and to replace only with the internal main girders and the abutments.

Figure 7.10 plots the two objectives for each of the optimum policies obtained. The plot shows a clear relationship of the average asset condition and the expected maintenance cost. As the average asset condition increases, illustrated by the decrease in value, the expected maintenance cost increases. This information combined with the constraints such as a maintenance budget constraint or critical asset condition constraint would be useful in determining the most appropriate of the optimised policies when making management decision.

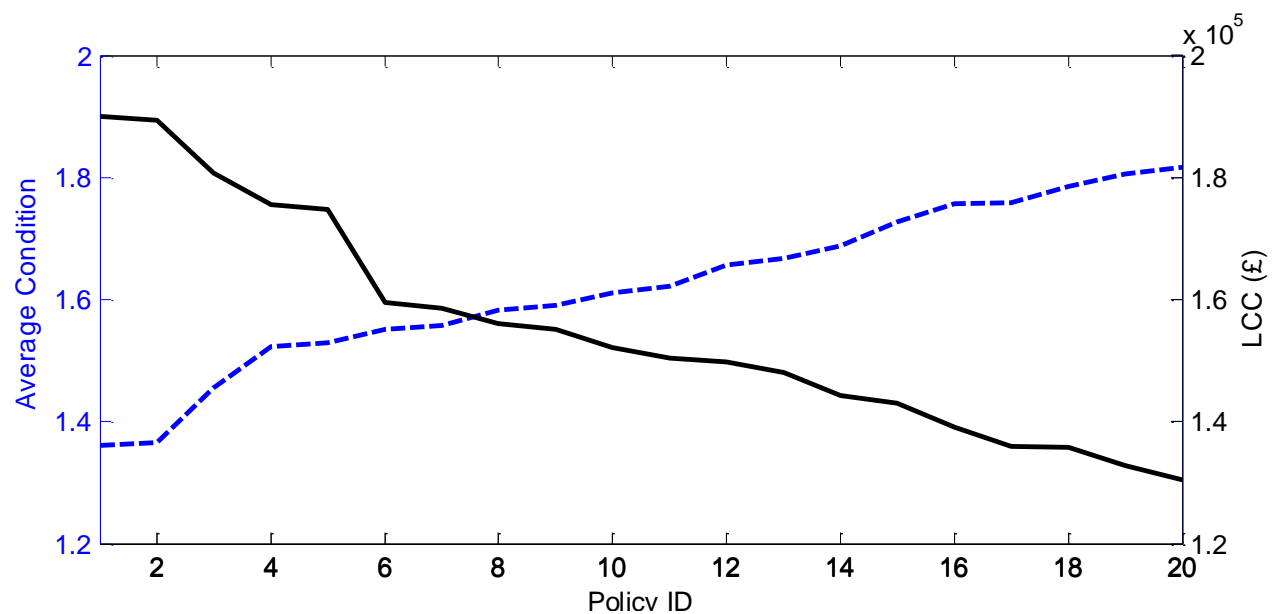

Figure 7.10: Asset average condition and expected maintenance costs for the 20 obtained optimum maintenance policies 


\subsubsection{Optimisation performance}

In the hybrid optimisation, the total computational analysis time includes the time to optimise the Markov model in order to generate the initial population for the PN optimisation. The time it takes to optimise the Markov model is 4.32 hours making the total optimisation time in the hybrid optimisation about 24.5 hours. This presents a decrease of about $21 \%$ in the optimisation time comparing with carrying out a normal optimisation. A normal optimisation starts with the initial population that is randomly generated and is not based on the optimisation results from the Markov model. The performance increasing comes from a faster convergence in the hybrid optimisation. The details of the processes, where both optimisations were run at the same time, are illustrated in Figure 7.11. Snapshots of their populations are compared at each generation. Both of the optimisation exercises were run up to the $40^{\text {th }}$ generation. In all the plots, the green squares represent the performance of the population of the normal optimisation, the red squares represent the population of the hybrid optimisation. It can be seen clearly that the hybrid optimisation converges at around after the $20^{\text {th }}$ generation whilst the normal optimisation converges at around the $30^{\text {th }}$ generation.

\begin{tabular}{|c|c|c|}
\hline & Hybrid optimisation & Normal optimisation \\
\hline Converged generation & 18 gens & 28 gens \\
\hline Total time taken & 24.5 hours & 31.1 hours \\
\hline
\end{tabular}

Table 7.9: Performance increase in the hybrid optimisation

Looking at the $40^{\text {th }}$ generation when both types of optimisations have converged, it can be seen that, the Pareto front of the hybrid optimisation is only a part of the front of the normal optimisation. This means that the normal optimisation would give a wider range of optimum solutions. Therefore, it is also important to realise that along with the performance increase in the optimisation time of the hybrid optimisation, there is also a chance of not covering the entire solution space. 

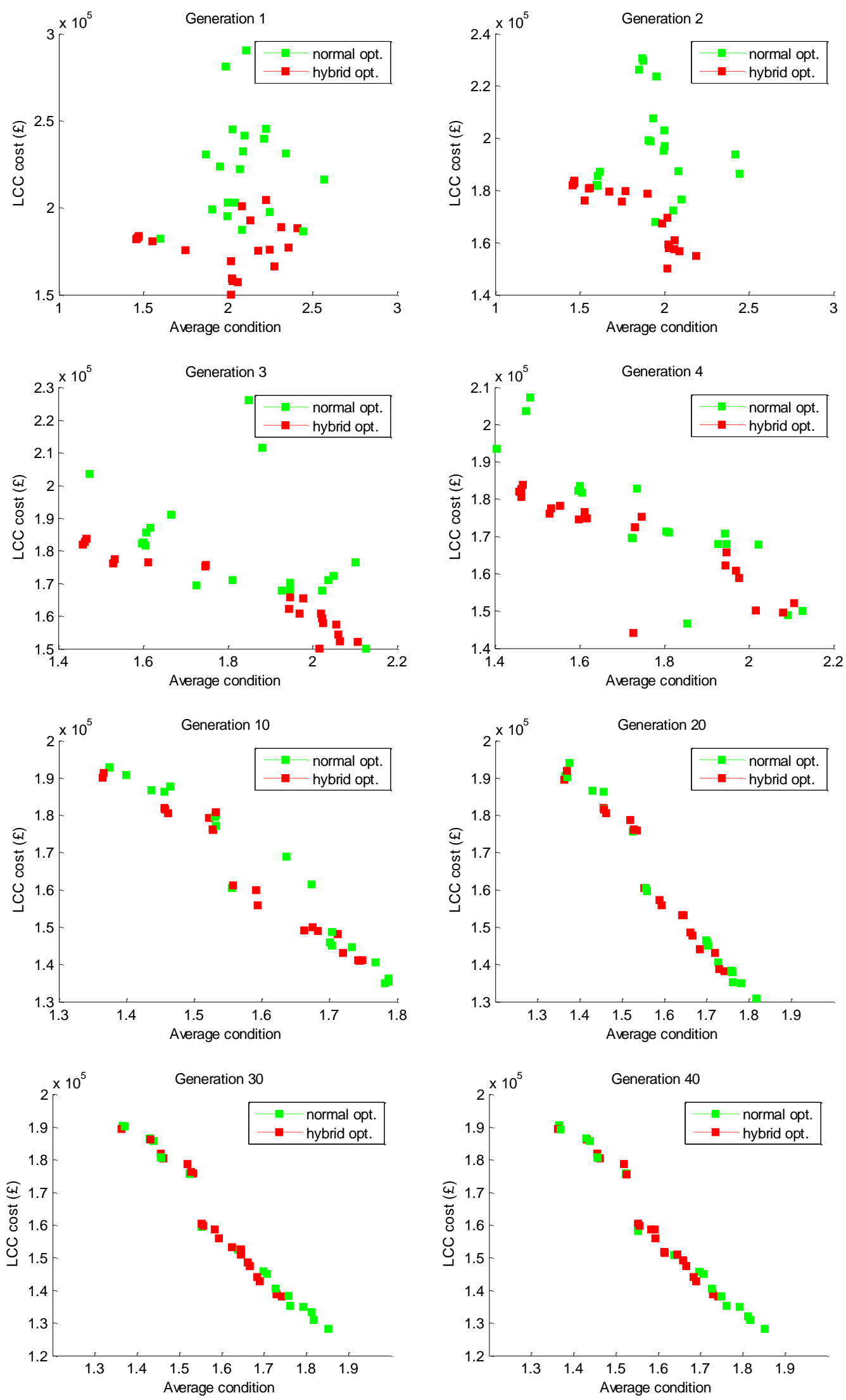

Figure 7.11: Comparison of the optimisation process of hybrid and normal optimisation 


\subsection{Summary}

An optimisation framework based on the Genetic Algorithm technique was used in this chapter as a decision making approach to select the best maintenance strategies. The optimisation was applied on both of the Markov and the Petri-Net bridge models. The optimisation is a multi-objective optimisation that looks for the maintenance policies that will produce the lowest expected maintenance cost whilst maximising the average condition of the asset.

This chapter starts with the optimisation of the Markov bridge model. The optimisation procedure including the evaluation of the objectives, variable coding, fitness, selection functions, etc., are discussed in detail. The results present 20 optimum maintenance policies. A policy was chosen from the set of optimised policies that shows an improved performance when compared with the current industry maintenance policy.

Following this, the optimisation of the Petri-Net bridge model is discussed. The optimisation for the PN model is a hybrid approach which utilises the final results from optimising the Markov bridge model. These results provide an initial population for the PN model optimisation. With this better initial population, the optimisation process was able to converge to the optimum solutions quicker, resulting in a performance increase of $21 \%$ faster in the optimisation time.

Overall, this chapter provides an optimisation framework for the bridge models. The optimal maintenance policies obtained represent a wide variety of maintenance strategies for the chosen asset. These policies would assist the decision making process by not only providing a number of solutions, but also justifying the decision to carry out repairs or let a component continue to deteriorate to the point where it is replaced. Combining this with the expert judgement and engineering knowledge, the optimum maintenance policy can be identified. 


\section{Chapter 8 - Conclusions}

\subsection{Summary and conclusions}

Bridges are an important part of the railway network. As bridges age, the management authorities are faced with the increasing pressure to keep the bridge in an acceptable condition accounting for budgetary constraints and the need to avoid service disruptions. The main objective of the research presented in this thesis is to develop a complete bridge asset state model. The key element of the model is to establish the deterioration characteristics of each of the bridge components. This has been achieved by the analyses of historical maintenance data. The model can give an accurate prediction of the asset future condition and can be used to demonstrate the effects of different maintenance strategies. An optimisation framework has been developed as a decision making approach to select the best maintenance policies. The optimum maintenance policy will be that which produces the lowest expected maintenance costs whilst maximising the average condition of the asset.

Following an extensive literature review on the existing bridge condition models, the needs for a more robust and detailed model were identified. The majority of bridge models have adopted the Markov chain method in predicting the deterioration process and future bridge element conditions. These models are simple and lack application and verification on real data. The Markov modelling approach also has several associated limitations such as: constant transition rates and the size of the model becomes 
unmanageable when the problem is complex. In addition when forming a bridge model in terms of its component conditions the complete asset model cannot be constructed by combining the component models, a completely new model form has to be generated. Other modelling techniques such as semi-Markov and reliability-based approaches have been developed. However, whilst having the potential to overcome the constant failure rate restriction, they have not provided a complete solution to the other issues in bridge asset modelling. Furthermore, all of the reviewed models are based on statistical analysis of condition ratings. It was identified that this data source is inadequate for a bridge degradation study.

The research proposed a study to understand the bridge deterioration based on analysing the recorded historical work done on bridge elements as an alternative to the use of condition ratings. By constructing a life history of each component and grouping similar components according to their structure types and materials, a statistical analysis was performed to model the characteristic behaviour of a given bridge element type. In this analysis, the Weibull distribution is fitted to the times for a component to reach different condition states (good, poor, very poor states). The study was carried out to model the degradation process for major bridge elements (main girders, deck, bearing, and abutment). The analysis suggested that the degradation rates of bridge elements are not constant and increases slowly over time. The degradation modelling forms the foundation to the development of a complete bridge model.

There are two bridge models that have been developed in this research. The first model is based on the Markov modelling approach, which is a widely accepted approach in bridge modelling. The second model is based on the Petri-Net method, which has never been applied to bridge modelling. In the development of the first model, a considerable level of detail has been introduced. The model accounts for the initial condition of bridge elements, material type, structure type, environment, inspection intervals, servicing intervals, repair strategy, opportunistic repair and repair scheduling times. All these model parameters can be varied to investigate the effects of different maintenance scenarios. The effects of a specified maintenance strategy is reflected in terms of the probabilities of the bridge 
elements being in different states at a given time in the future; and the expected maintenance cost over the entire prediction period. Application of the Markov bridge model has provided an insight into this modelling technique, understanding both the capabilities and the limitations and providing a bridge modelling framework that can be used for the validation of the second bridge model developed.

It was shown that, even though the Markov model is capable of detailed modelling, a more flexible and robust approach is required. Therefore, a second bridge model was developed which is based on the Petri-Net (PN) approach. The PN approach is increasingly being used to model dynamic systems, and in this research, a number of modifications to the traditional PN concept have been made to suit the problem in bridge asset management. The PN model not only keeps the level of complexity that was developed in the Markov model but also further increases the level of modelling detail. One of the most important features is the capability of incorporating the non-constant deterioration rates of bridge elements. Furthermore, the PN model models the degradation process of the protective coating for metal elements. It also realises some repairs are ineffective after a number of times carried out and the planning of repairs accounts for different maintenance schedules according to the criticality of the asset. A Monte Carlo simulation was used to simulate the model and the statistics collected indicate the performance of the bridge and its elements.

The results and performances of the two models can be compared by applying, as near as possible, the same maintenance policy on the same asset. It is worth noting that, with the increasing level of details in the PN model, the results obtained from the two models will not be exactly the same, however it is expected that they should be very close to each other. The comparison study confirmed that both models predict very similar future average conditions. This validates the PN approach to the modelling of bridge assets. The comparison also shows that the PN bridge model predicts more informative on the asset condition profiles in the near future. This information can be useful in the way that the inspection and maintenance can be allocated accordingly. Moreover, the PN model is more efficient in the execution time when a system contains many components. 
These models provide a means of investigating the effects of different maintenance policies. Based on the bridge models, an optimisation framework has been developed to find the optimum maintenance policies such that the average asset condition is maximised whilst the expected maintenance cost is minimised. Optimisation studies were carried out for both models and the results present a set of 20 optimum maintenance policies. A policy was chosen from the set of optimised policies that shows an improved performance when compared with the current industry maintenance policy. It was realised that for a complex optimisation problem, where many system evaluations will be required, that the efficiency of the optimisation process is important. To address this, the research developed a hybrid optimisation framework, which utilises the optimisation results from the Markov bridge model as initial guesses for the optimisation of the PN bridge model. An $21 \%$ reduction in the optimisation time was achieved using the hybrid optimisation. The maintenance optimisation provides a wide range of optimum policies that would assist the decision making process and combined with the engineering judgements, the optimum policy for a bridge asset can be selected.

The proposed bridge model delivers a tool for the bridge management authorities and decision makers to assess the degradation process and investigate the effects of different

maintenance policies on bridge assets. The model can be employed to determine cost-effective bridge maintenance strategies while maintaining a desirable bridge condition.

\subsection{Research contributions}

Based on the current developments, this research makes a number of contributions:

- Better understanding of bridge asset management needs: This research has reviewed a number of studies and the shortcomings in current modelling approaches have been identified. The needs for a new approach, as well as the several practical modelling requirements, were understood through meetings and discussions with bridge experts and the bridge management authority. 
- The use of historical maintenance data: Instead of bridge condition rating data, the research used historical work done data when formulating the degradation models. The maintenance effects is captured and analysed with this data.

- The estimation of the degradation process for a single component given a degradation process of a group of similar components: Many bridge elements exist in quantities greater than one on a structure. In this case the maintenance work carried out is often only attributed to the component type and does not specify which one. The degradation analysis then gives the degradation characteristic of the set of components. A method based on simulation was developed in this research to estimate the degradation process of each individual component using this data.

- Better understanding of the degradation processes of several major bridge elements: the study has presented the analysis for metal underbridge' major elements such as: metal girder, concrete deck, metal deck, timber deck, metal bearing, and masonry abutment.

- The consideration of many factors that affects the deterioration and maintenance planning process: Environment, the increasing deterioration rates of bridge elements, the degradation process of protective coatings for metal elements, inspection intervals, servicing intervals, level of intervention, opportunistic repair and repair scheduling times are the factors included in the models proposed.

- The application of the Petri-Net approach to bridge assets modelling: The main advantage of this research is the development of the Petri-Net bridge model. The flexibility and capability of the approach offers a more robust modelling for bridge asset management, its advantages have been presented and discussed throughout the thesis. The model allows easy expansion (i.e. more detail modelling) and the opportunity to expand the model to system level with integrated asset modelling.

- The application of the hybrid optimisation: The optimisation performance increases with the use of the 
hybrid optimisation approach resulting in a shorter optimisation times.

\subsection{Future works}

Despite the capabilities and benefits of the research, there are several areas in which further work can be carried out:

- The current assumption of the bridge models is that repairs are perfect i.e. maintenance restores the component condition to the 'as good as new' condition. More research is needed in order to determine the effect of the repair on the component condition as well as the degradation process after each time the repair is carried out.

- It is anticipated that the analysis of more complex problems would require more computational effort. Therefore future work should be focused on improving the efficiency of the analysis, especially the efficiency of the computer coding to reduce the solution time.

- There are only three types of interventions considered in this research due to the limited availability of the historical data. With more data becomes available, more specific works can be modelled, this means that more types of component defects are considered. For this purpose, extra model states can be added to accommodate the increasing level of modelling detail.

- In the research presented in this thesis, the Genetic Algorithm (GA) was used as the optimisation method. This has proven to be sufficient enough for the optimisation problem presented. However, other advanced GAs and hybrid heuristic techniques provide potential alternatives. Research could be performed to improve the search ability and computational efficiency of such approaches.

- Future work should focus on the development of bridge models for other asset types (e.g. overbridges) and ultimately, the development of a complete network model which allows the asset management of entire railway network to be modelled as a system. 


\section{References}

AGRAWAL, A. K. \& KAWAGUCHI, A. 2009. Bridge element deterioration rates. New York State Department of Transportation.

AGRAWAL, A. K., KAWAGUCHI, A. \& CHEN, Z. 2010. Deterioration rates of typical bridge elements in New York. Journal of Bridge Engineering, 15, 419-429.

ANDREWS, J. 2013. A modelling approach to railway track asset management. Proceedings of the Institution of Mechanical Engineers, Part F: Journal of Rail and Rapid Transit, 227, 56-73.

ANDREWS, J. D. \& MOSS, T. R. 2002. Reliability and Risk Assessment, London and Bury St Edmunds, UK, Professional Engineering Publishing Limited.

BAUSE, F. \& KRITZINGER, P. S. 2002. Stochastic Petri Nets, Springer.

BU, G., SON, J., LEE, J., GUAN, H., BLUMENSTEIN, M. \& LOO, Y. 2013. Typical deterministic and stochastic bridge deterioration modelling incorporating backward prediction model. Journal of Civil Structural Health Monitoring, 1-12.

BUTT, A. A., SHAHIN, M., CARPENTER, S. \& CARNAHAN, J. Application of Markov process to pavement management systems at network level. 3rd International Conference on Managing Pavements, 1994. 159-172.

CESARE, M. A., SANTAMARINA, C., TURKSTRA, C. \& VANMARCKE, E. H. 1991. Modelling Bridge Deterioration With Markov Chains. Journal of Transportation Engineering, 118, 2237.

CESARE, M. A., SANTAMARINA, C., TURKSTRA, C. \& VANMARCKE, E. H. 1992. Modeling bridge deterioration with Markov chains. Journal of Transportation Engineering, 118, 820833.

CHIOLA, G., MARSAN, M. A., BALBO, G. \& CONTE, G. 1993. Generalized stochastic Petri nets: A definition at the net level and its implications. Software Engineering, IEEE Transactions on, 19, 89-107.

CIARDO, G., MUPPALA, J. \& TRIVEDI, T. SPNP: stochastic Petri net package. Petri Nets and Performance Models, 1989. PNPM89., Proceedings of the Third International Workshop on, 11-13 Dec 1989 1989. 142-151.

DESTEFANO, P. D. \& GRIVAS, D. A. 1998. Method for estimating transition probability in bridge deterioration models. Journal of Infrastructure Systems, 4, 56-62. 
FONSECA, C. M. \& FLEMING, P. J. Genetic Algorithms for Multiobjective Optimization: FormulationDiscussion and Generalization. ICGA, 1993. 416-423.

FRANGOPOL, D. M., KONG, S. J. \& GHARAIBEH, S. E. 2001. Reliability-based life-cycle management of Highway bridges. Journal of Computing in Civil Engineering, 15, 27-34.

FRANGOPOL, D. M., YANG, S.-I. \& NEVES, L. C. 2006. Optimum maintenance strategy for deteriorating bridge structures based on lifetime functions. Engineering Structures, Vol. 28, pg. 196-206.

GIRAULT, C. \& VALK, R. 2002. Petri nets for systems engineering: a guide to modeling, verification, and applications, Springer.

HALCROW 2011. Bridges Tier 2 Whole Life Cycle Cost Model Phase 1. In: NETWORKRAIL (ed.) Bridges Tier 2 Whole Life Cycle Cost Model.

HAUPT, R. L. \& HAUPT, S. E. 2004. Practical genetic algorithms, Wiley-Interscience.

HEARN, G., THOMPSON, P. D. \& HYMAN, W. 2010. Framework for a National Database System for Maintenance Actions on Highway Bridges. WASHINGTON, D.C.

HOLLAND, J. H. 1975. Adaptation in natural and artificial systems: an introductory analysis with applications to biology, control, and artificial intelligence, University of Michigan Press.

HOLLIDAY, M. A. \& VERNON, M. K. 1987. A generalized timed Petri net model for performance analysis. Software Engineering, IEEE Transactions on, 1297-1310.

IBE, O. C. 2009. Markov Processes for Stochastic Modeling, Academic Press.

JENSEN, K. 1996. Coloured Petri Nets: Basic Concepts, Analysis Methods and Practical Use, Springer.

JENSEN, K. \& KRISTENSEN, L. M. 2009. Coloured petri nets, Springer.

JIANG, Y. \& SINHA, K. C. 1989. Bridge service life prediction model using the Markov chain. Transportation Research Record, 24-30.

KLEINER, Y. 2001. Scheduling inspection and renewal of large infrastructure assets. Journal of Infrastructure Systems, 7, 136-143.

MALHOTRA, M. \& TRIVEDI, K. S. 1995. Dependability modeling using Petri-nets. Reliability, IEEE Transactions on, 44, 428440.

MARSAN, M. A., BALBO, G., CONTE, G., DONATELLI, S. \& FRANCESCHINIS, G. 1998. Modelling with generalized stochastic petri nets. ACM SIGMETRICS Performance Evaluation Review, 26, 2.

MISHALANI, R. G. \& MADANAT, S. M. 2002. Computation of infrastructure transition probabilities using stochastic 
duration models. Journal of Infrastructure Systems, 8, 139148.

MISHALANI, R. G. \& MADANATE, S. M. 2002. Computational of infrastructure transition probabilities using stochastic duration models. Journal of Infrastructure, 8, 139-148.

MOLLOY, M. K. 1982. Performance Analysis Using Stochastic Petri Nets. Computers, IEEE Transactions on, C-31, 913-917.

MORCOUS, G. 2006. Performance Prediction of Bridge Deck Systems Using Markov Chains. Journal of Performance of Constructed Facilities, Vol. 20, 146-155.

NETWORK RAIL 2004a. NR/SP/CIV/017: Examination of bridges and culverts.

NETWORK RAIL 2004b. Structures Condition Marking Index Handbook for Bridges (formerly RT/CE/C/041).

NETWORK RAIL 2007a. Network Rail Annual Return 2007. In: RAIL, N. (ed.).

NETWORK RAIL 2007b. Network Rail Strategic Business Plan. Asset Management.

NETWORK RAIL 2009. The use of protective coatings and sealants (Guidance Note).

NETWORK RAIL 2010a. Asset Crititicality Risk 8. In: NIGEL RICKETTS (ed.) Network Rail - 10 Step Plan.

NETWORK RAIL 2010b. Structure Unit Rates From Tier 1 Models.

NETWORK RAIL 2011. Asset Policy Developement. Asset Policy Developement.

NETWORK RAIL 2013. Strategic Business Plan for England \& Wales for CP5.

NG, S. \& MOSES, F. 1996. Prediction of bridge service life using time-dependent reliability analysis. Bridge management, 3 , 26-32.

NG, S. \& MOSES, F. 1998. Bridge deterioration modelling using semi-Markov theory. Structure Safety and Reliability, 1-3, 113-120.

NOORTWIJK, J. M. V. \& KLATTER, H. E. 2004. The use of lifetime distribution in bridge maintenance and replacement modelling. Computers and Structures, 82, 1091-1099.

OFFICE OF RAIL REGULATION 2007. Annual assessment of Network Rail 2006-07.

ORTIZ-GARCÍA, J. J., COSTELLO, S. B. \& SNAITH, M. S. 2006. Derivation of transition probability matrices for pavement deterioration modeling. Journal of Transportation Engineering, 132, 141-161.

PETRI, C. A. 1963. Fundamentals of a Theory of Asynchronous Information Flow. Proc. of IFIP Congress 62. --Amsterdam: North Holland Publ. Comp., 386-390.

PRESCOTT DR, A. J. 2012. A Railway Track Ballast Maintenance and Inspection Model for Multiple Track Sections. In Proceedings of PSAM 11 (Probablistic Safety Assessment 
and Management) / ESREL 2012 (European Safety and Reliabilty Conference). Helsinki, Finland.

REISIG, W. \& ROZENBERG, G. 1998. Lectures on Petri Nets I: Basic Models: Advances in Petri Nets, Springer.

ROBELIN, C.-A. \& MADANAT, S. M. 2007. History-dependent bridge deck maintenance and replacement optimization with Markov decision processes. Journal of Infrastructure Systems, 13, 195-201.

RT/CE/C/002 2002. Application and Reapplication of Protective Treatment to Railtrack Infrastructure. Network Rail.

RT/CE/S/039 2002. Specification RT98-Protective treatments for Railtrack Infrastructure. Network Rail.

SAMHOURI, M. S. An intelligent opportunistic maintenance (OM) system: a genetic algorithm approach. 2009. IEEE, 60-65.

SANDERS, D. \& ZHANG, Y. 1994. Bridge deterioration models for states with small bridge inventories. Transportation Research Record.

SCHERER, W. T. \& GLAGOLA, D. M. 1994. Markovian models for bridge maintenance management. Journal of Transportation Engineering, 120, 37-51.

SCHNEEWEISS, W. G. 1999. Petri nets for reliability modeling:(in the fields of engineering safety and dependability), LiLoLeVerlag.

SIANIPAR, P. R. M. \& ADAMS, T. M. 1997. Fault-tree model of bridge element deterioration due to interaction. Journal of Infrastructure Systems, 3, 103-110.

SOBANJO, J., MTENGA, P. \& RAMBO-RODDENBERRY, M. 2010. Reliability-based modeling of bridge deterioration hazards. Journal of Bridge Engineering, 15, 671-683.

SOBANJO, J. O. 2011. State transition probabilities in bridge deterioration based on Weibull sojourn times. Structure and Infrastructure Engineering, 7, 747-764.

STEPHENS, K. S. 2012. Reliability Data Analysis with Excel and Minitab, ASQ Quality Press.

THOFT-CHRISTENSEN, P. 1999. Estimation of Bridge Reliability Distributions. Current and future trends in bridge design, construction and maintenance, 15-25.

THOMAS, O. \& SOBANJO, J. 2013. Comparison of Markov Chain and Semi-Markov Models for Crack Deterioration on Flexible Pavements. Journal of Infrastructure Systems, 19, 186-195.

VOLOVOI, V. 2004. Modeling of system reliability Petri nets with aging tokens. Reliability Engineering \& System Safety, 84, 149-161.

YANG, S.-I., FRANGOPOLA, D. M., KAWAKAMIB, Y. \& NEVES, L. C. 2006. The use of lifetime functions in the optimization of interventions on existing bridges considering maintenance and failure costs. Reliability Engineering and System Safety, Vol. 91, pg. 698-705. 
YANG, Y. N., PAM, H. J. \& KUMARASWAMY, M. M. 2009. Framework Development of Performance Prediction Models for Concrete Bridges. Journal of Transportation Engineering, $135,545-554$. 


\section{Appendix A Data analysis and degradation study}

\section{A-1 Data preparation}

Figure A.1 shows the five separate datasets used in this research and their information fields. The aim was to combine these datasets into a single working database where the information can be easily queried for any analysis. There are three main steps in the data preparation process: step one and two was to cleanse and combine all these databases, step three were to filter out the relevant information from the data.

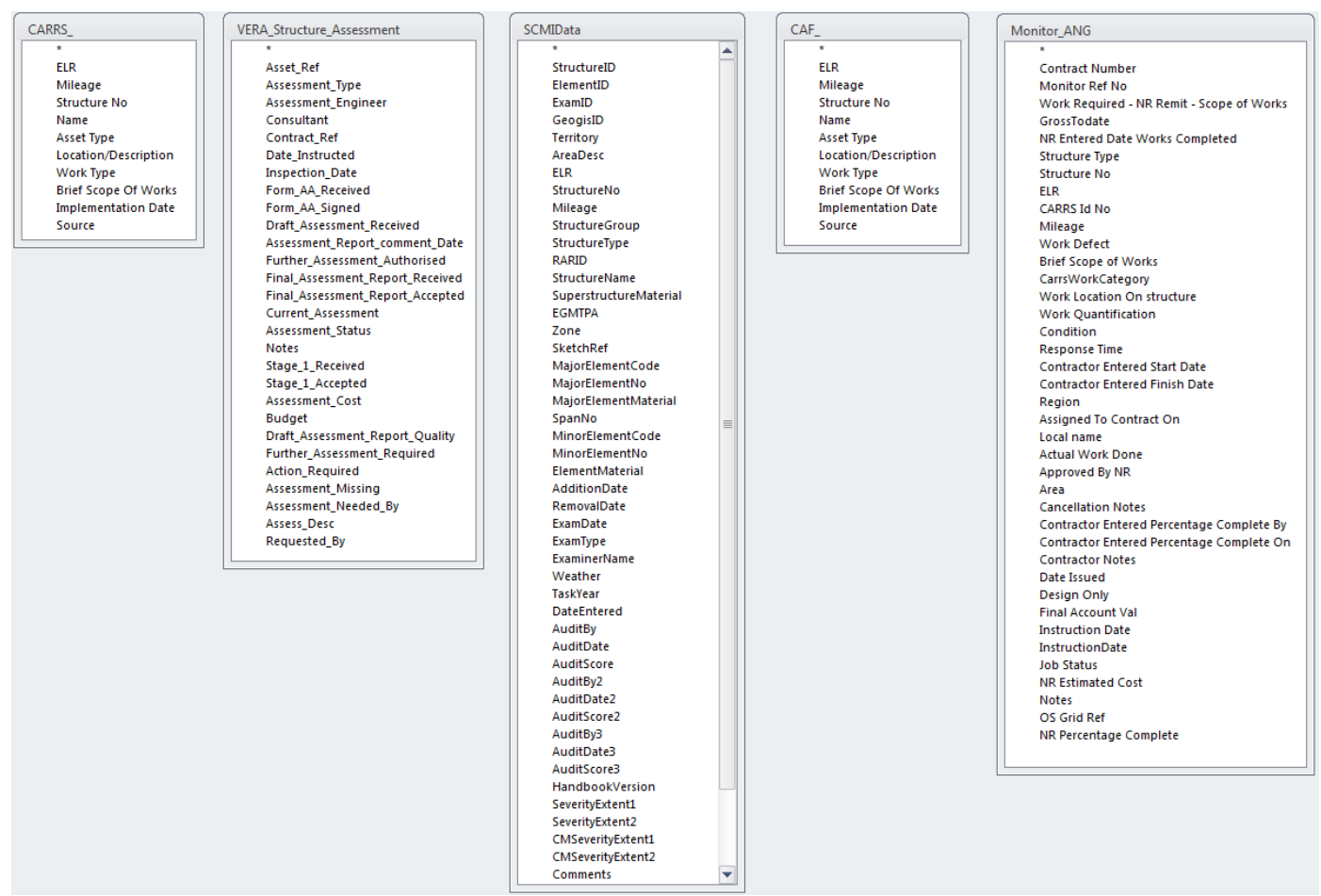

Figure A.1: All the dataset fields

\section{i. Data cleansing}

This step was to clean each dataset from records that contains non-useful information such as blank records (e.g. records contains no work done information); records were incorrectly entered (e.g. records contains date in the future). Details of the actions for each dataset are given in the Table A.1 below. 


\begin{tabular}{|l|l|}
\hline \multicolumn{1}{|c|}{ Dataset } & \multicolumn{1}{c|}{ Cleaning action } \\
\hline CARRS & $\begin{array}{l}\text { Select only records related to bridges by looking for "*bridge*" } \\
\text { in "Asset Type" field. }\end{array}$ \\
\hline VERA & $\begin{array}{l}\text { Remove records }(15,007) \text { with blank inspection date. } \\
\text { Remove records }(5,936) \text { were recorded at 01/01/1900, these } \\
\text { records were assumed that incorrectly entered resulting in the } \\
\text { software default date 01/01/1900. } \\
62 \% \text { of the records were removed. }\end{array}$ \\
\hline SCMI & $\begin{array}{l}\text { No actions were carried out. } \\
\text { e.g. the location of a bridge on the Engineering Line Reference } \\
\text { (ELR) were recorded in chains instead of mileages. Therefore a } \\
\text { script was written to look for and convert any chain unit into } \\
\text { mileage. This step is important to ensure the consistency across } \\
\text { all the datasets as the ELR and Mileage are the unique number } \\
\text { to identify a particular asset. }\end{array}$ \\
\hline MONITOR & $\begin{array}{l}\text { Select only records related to bridges by looking for "*bridge*" } \\
\text { in "Structure Type" field. } \\
\text { Remove records with 'Job Status' marked as 'cancelled'. }\end{array}$ \\
\hline
\end{tabular}

Table A.1: Cleaning actions

\section{ii. Data combining}

This step combined all the datasets above into one relational database that contains only necessary information for the study. Figure A. 2 shows the relationship between all the datasets before combination and theirs useful fields. Comparing Figure A.1 and Figure A.2, it can be seen that the number of fields has reduced, this is because only fields contain useful information were combined together, this helps to reduce the size of the dataset and also eliminates the clustering of non-related data.

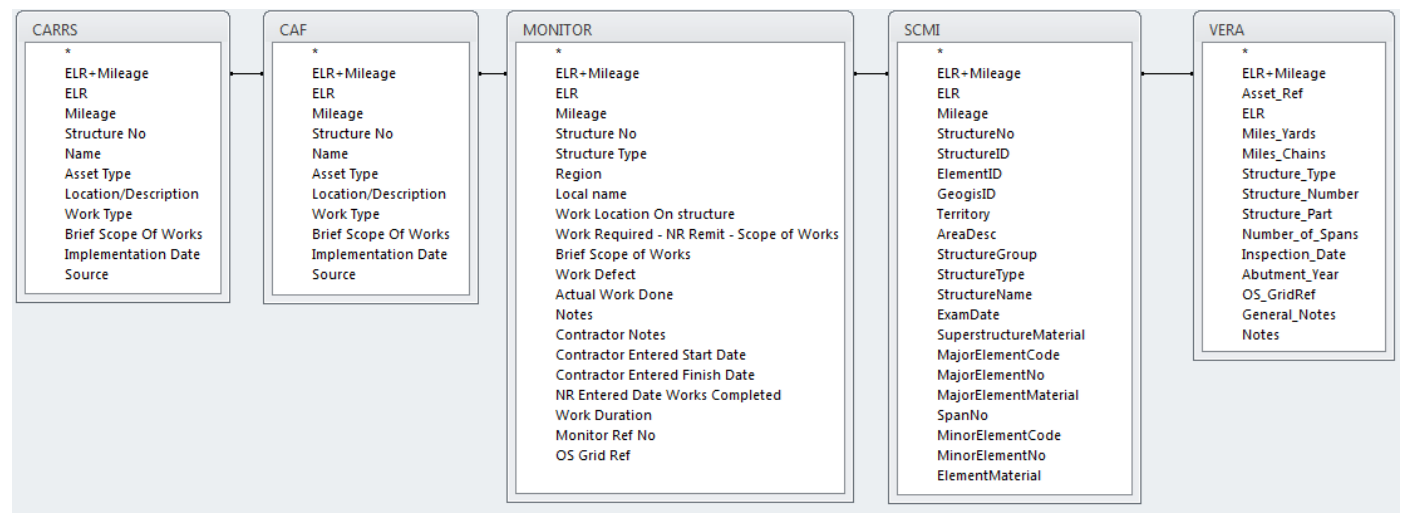

Figure A.2: Combining all databases

After the concatenation, the combined database contains the information as illustrated in Figure A.3. There are several key information in this working database: 
- The first is the bridge information includes the location of the bridge, bridge name and the bridge structure type.

- The second is the description of the work has been carried out on that particular bridge.

- The third is the date when the work was carried out or the date when the bridge was inspected.

- The fourth is the associated costs of the intervention, including the work quantification, estimate costs and gross costs.

- The fifth is the bridge element information such as element material, element arrangement in a bridge and the SCMI score.

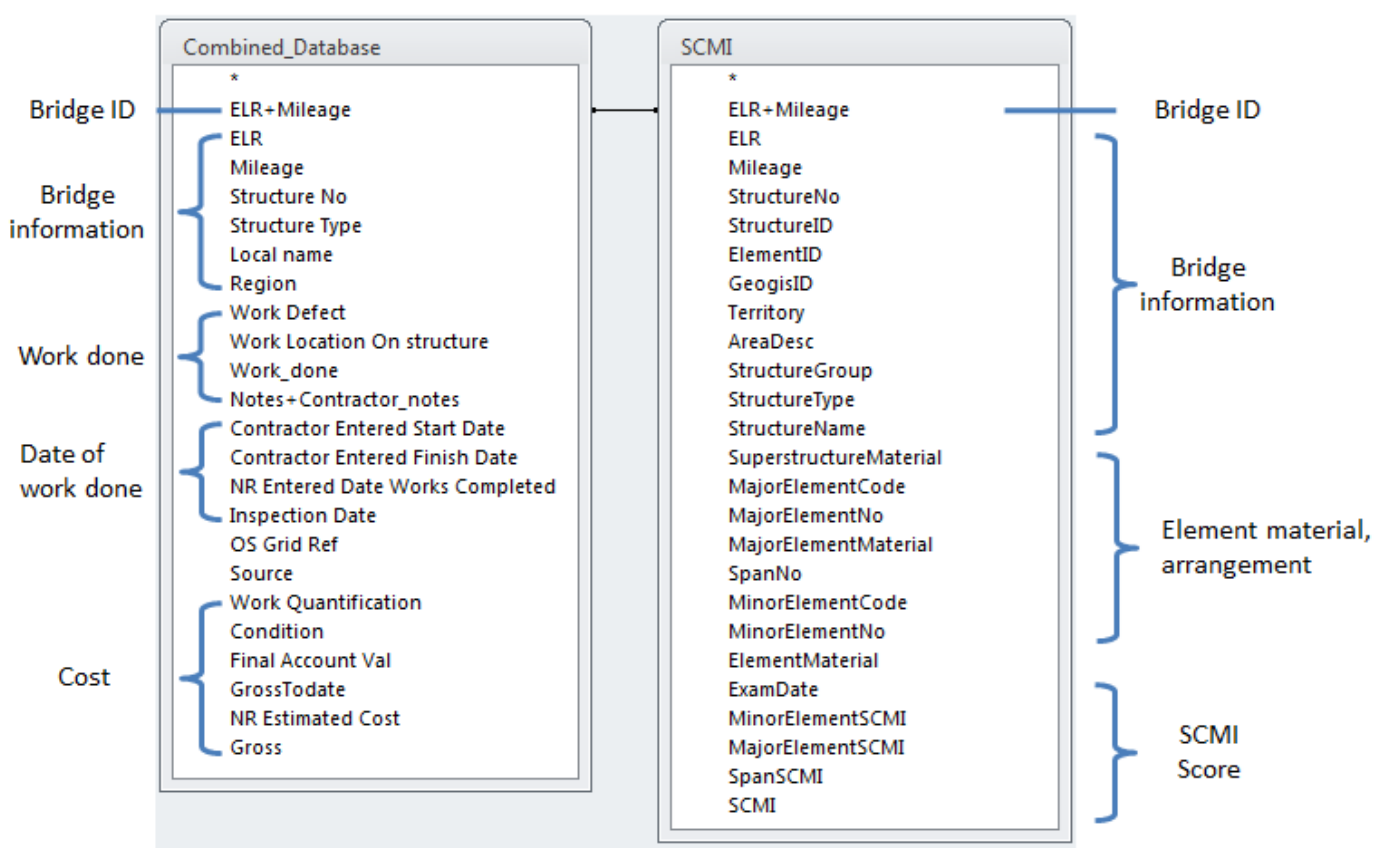

Figure A.3: Combined database

\section{Problems and assumptions}

Because the data was combined from different datasets, there are different ways in which the bridge was identified, for example, SCMI uses 'StructureID'; VERA uses 'Asset_Reference' to identify a bridge. A unique number that is made of ELR and Mileage were used to uniquely identify a bridge. The reason for this is because firstly ELR and Mileage are available in all the datasets and secondly ELR (Engineering Line Reference) and Mileage uniquely defined the location of a bridge.

Even though ELR and Mileage are available in all datasets, in CAF dataset, as mentioned earlier, the location of a bridge on a track 
line was sometimes recorded in chain instead of mileage, therefore these values were converted back into mileage to ensure the compatibility between all the datasets.

For the information about the date when the work was carried out, the 'contractor entered date' where available were always be used over 'Network Rail entered date', this is because upon the examination of the data, 'Network Rail entered dates' are always much later than the dates entered by the contractors and are often associated with the dates where the account for the job was finalised, therefore it was thought that the contractor's date were more accurate of when the work started and finished.

\section{iii. Data filtering}

As discussed in the previous section, the nature of the poorly structured data means that the historical work done data are not descriptive words but rather sentences or paragraphs describing the work carried out. This makes it very hard to quickly obtained information of the type of repair, bridge component that was repaired, etc. A search function was built that searches for keywords and combination of keywords however, the search can only be effective and accurately filter a small percentage ( $10 \%)$ of the data. Therefore the rest of the data were examined manually in order to categorise them appropriately. Figure A.4 demonstrates this process, the work description fields were read and the data entry is manually categorised, the last column is added to original data and indicates the categorised specific work.

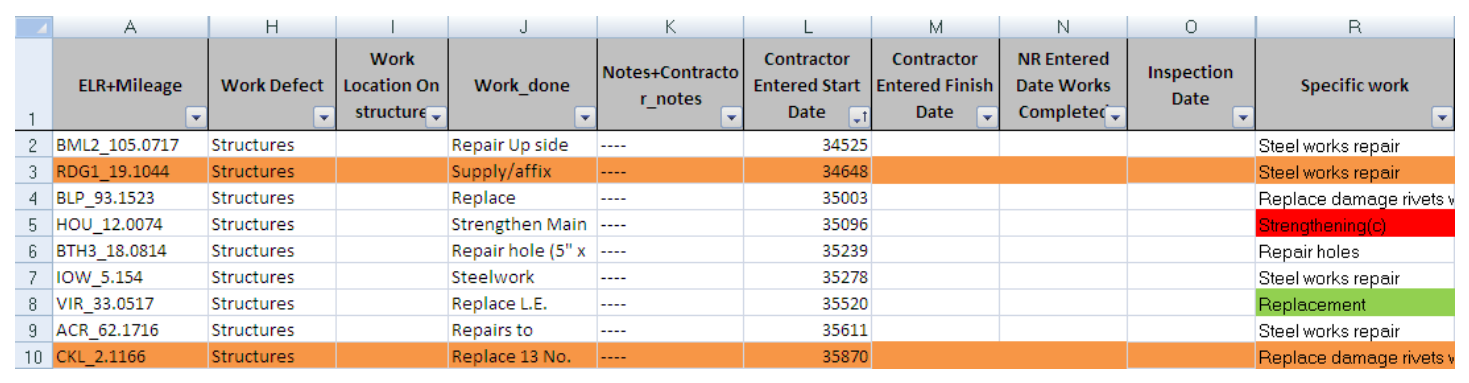

Figure A.4: Categorising data 


\section{A-2 Typical metal underbridge elements}

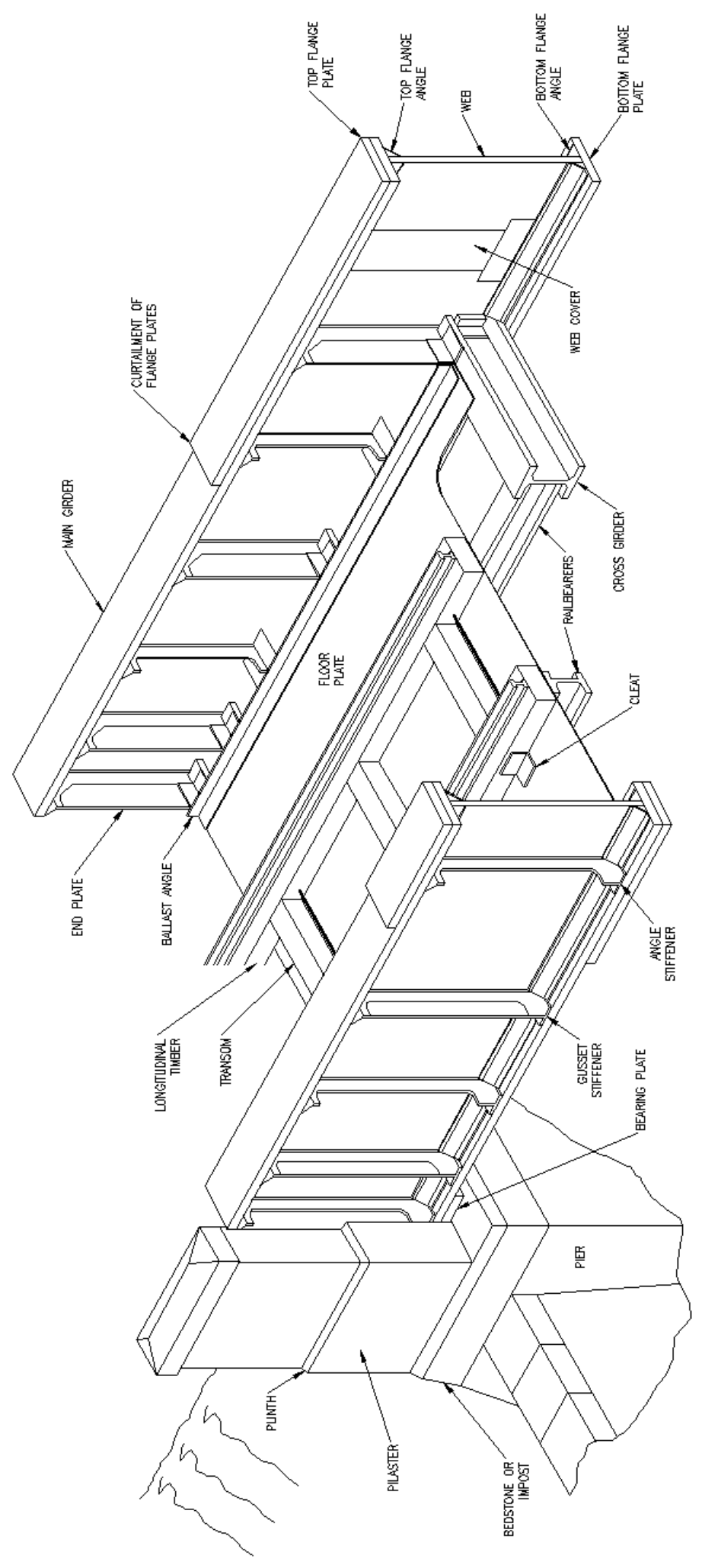

Figure A.5: Metal Underbridge (Network Rail, 2011) 


\section{A-3 Element condition states}

The condition of an element is decided based on the significant of a defect discovered. Network Rail's standards uses the term Severity and Extent (SevEx) to quantify a component defect. The definitions of the SevEx (Network Rail, 2004b) of defects on metallic element are given in Figure A.6. The SevEx shows the seriousness of defects and also give the idea of the degradation process on a metal bridge element. A given combination of severity and extent (e.g. A2) indicates a particular type of defect, however many defects can be grouped together indicating the same level of degradation i.e. a certain condition state. By studying the combination of them different levels of degradation can be seen.

Table A.2 shows all the combinations of SevEx and the four condition states for a metal element.

Element with no visible defects (B2) to localise corrosion/ loss of section up to $5 \mathrm{~mm}$ deep ( $C 2$ or B3), it is only minor defects and would be considered to be in an 'as new' state.

Element with minor corrosion defects is when the corrosion/loss of section is less than $1 \mathrm{~mm}$ deep but occupies up to $10 \%$ of the surface of the element; up to the corrosion defects that more than $10 \mathrm{~mm}$ deep and occupies more than $50 \%$ surface area of the element. The element is considered to be in a good state.

Major corrosion is when localised corrosion / loss of section have taken out the full thickness of the element section up to the case when the loss of the full thickness of the section is not localised and has spread up to $5 \%$ surface area of the element. This is poor state.

The very poor state is when the element experiences major distortion i.e. when the element is torn, fractured, buckled or permanently distorted, this type of defects often seriously affects the structural capability of the elements and would generally require immediate attention and a complete replacement of the failed component.

For components of different materials (concrete, timber, masonry), the definition of the SevEx and condition states are also given below (Figure A.7 to A.9 and Table A.3 to Table A.5). 
In general, by studying the levels of defects, it can be seen that a bridge element can be in 'as new' condition, good condition, poor condition or very poor condition and the associated interventions for components in good to very poor condition are minor repair, major repair and renewal respectively.

\begin{tabular}{|c|l|}
\hline Severity rating & \multicolumn{1}{c|}{ Definition } \\
\hline A & No visible defects to metal \\
\hline B & Corrosion/loss of section< 1mm deep \\
\hline C & Corrosion/loss of section 1mm up to 5mm deep \\
\hline D & Corrosion/loss of section $>5 \mathrm{~mm}$ up to 10mm deep \\
\hline E & Corrosion/loss of section $>10 \mathrm{~mm}$ but not through section \\
\hline F & Corrosion/loss of section to full thickness of section \\
\hline & \multicolumn{1}{c|}{$\begin{array}{c}\text { Choose most extensive from: } \\
\text { Tears, fracture, cracked welds } \\
\text { Buckling, permanent distortion or displacement }\end{array}$} \\
\hline
\end{tabular}

Severity rating $\mathrm{G}$ relates to a condition that would normally merit immediate notification to Network Rail if known to be a new defect.

\begin{tabular}{|c|l|l|}
\hline Extent & \multicolumn{2}{|c|}{ Definition } \\
\hline 1 & No visible defects \\
\hline 2 & $\begin{array}{l}\text { Localised defect due to local circumstances (such as } \\
\text { isolated damage caused by a single bridge strike or } \\
\text { isolated water leakage) }\end{array}$ \\
\hline 3 & & $<5 \%$ \\
\hline 4 & Percentage of surface of the & $5 \%$ up to $10 \%$ \\
& element occupied by defect & $>10 \%$ up to $50 \%$ \\
\hline 6 & & $>50 \%$ \\
\hline
\end{tabular}

Table 2C.14: Severity and extent ratings for metal

Figure A.6: Severity and Extent definition (Network Rail, 2004b)

\begin{tabular}{|c|c|c|c|c|c|c|c|}
\hline \multicolumn{7}{|l|}{ METAL } & \multirow[b]{4}{*}{ Defect Level } \\
\hline & \multicolumn{6}{|c|}{ Extent } & \\
\hline \multirow{2}{*}{$\frac{\text { Severity }}{A}$} & 1 & 2 & 3 & 4 & 5 & 6 & \\
\hline & 0 & & & & & & \\
\hline B & & 1 & 2 & 2.5 & 3 & 3.5 & Minor or no defect \\
\hline C & & 2 & 3.5 & 4 & 5 & 6 & Minor corrosion \\
\hline \multirow{2}{*}{$\begin{array}{l}\text { D } \\
\text { E }\end{array}$} & & 3 & 4 & 5 & 6.5 & 8.5 & \multirow{2}{*}{$\begin{array}{l}\text { Major corrosion, loss of section, } \\
\text { fracture, crack welds }\end{array}$} \\
\hline & & 4 & 5 & 7 & 8.5 & 9 & \\
\hline \multirow{2}{*}{$\mathbf{F}$} & & 5 & 7 & 9 & 9.5 & 10 & \multirow{2}{*}{$\begin{array}{l}\text { Major loss of section, buckling, } \\
\text { permanent distortion }\end{array}$} \\
\hline & & 5 & 8.5 & 9 & 9.5 & 10 & \\
\hline
\end{tabular}

Table A.2: Severity and extent scores for Metal 


\begin{tabular}{|c|c|c|}
\hline Severity & \multicolumn{2}{|c|}{ Definition } \\
\hline A & No visible defects & \\
\hline B & $\begin{array}{l}\text { Choose most exte } \\
\text { - Surface damage/chips/scrapes/minor spalls } \\
\text { - Indications of wetness/presence of water } \\
\text { (define as percolation, run down etc } \\
\text { excluding exposure to rainfall) } \\
\text { Staining (from aggregates, tying wire, nails or } \\
\text { other minor metallic debris) }\end{array}$ & $\begin{array}{l}\text { sive from: - } \\
\text { Cracking < Imm wide without evidence } \\
\text { of corrosion of reinforcement. }\end{array}$ \\
\hline C & $\begin{array}{l}\text { Choose most exte } \\
\text { - Spalling (no exposed reinforcement) } \\
\text { without evidence of corrosion of } \\
\text { reinforcement. }\end{array}$ & $\begin{array}{l}\text { Isive from: - } \\
\text { Cracking I } \mathrm{mm} \text { wide or greater without } \\
\text { evidence of corrosion of } \\
\text { reinforcement. }\end{array}$ \\
\hline D & $\begin{array}{l}\text { Choose most exte } \\
\text { - Spalling (no exposed reinforcement) with } \\
\text { evidence of corrosion of reinforcement } \\
\text { (excludes staining from metallic debris, see } \\
\text { notes on severity B). Includes delamination \& } \\
\text { drumminess, see notes on severity D. }\end{array}$ & $\begin{array}{l}\text { sive from: - } \\
\text { Cracking of any width (no exposed } \\
\text { reinforcement) with evidence of } \\
\text { corrosion of reinforcement (excludes } \\
\text { staining from metallic debris, see notes on } \\
\text { severity B). }\end{array}$ \\
\hline $\mathrm{E}$ & \multicolumn{2}{|l|}{ Secondary reinforcement exposed } \\
\hline $\mathrm{F}$ & \multicolumn{2}{|l|}{ Primary reinforcement exposed } \\
\hline G & \multicolumn{2}{|l|}{$\begin{array}{l}\text { Structural damage to element. (Includes } \\
\text { permanent distortion/displacement) }\end{array}$} \\
\hline
\end{tabular}

\begin{tabular}{|c|l||l|}
\hline Extent & \multicolumn{1}{|c|}{ Definition } \\
\hline & \multicolumn{1}{|c|}{ For all severities } & \multicolumn{1}{|c|}{ For cracking of severity' B, C \& D } \\
\hline I & No visible defects & No visible defects \\
\hline 2 & $\begin{array}{l}\text { Localised defect due to local circumstances } \\
\text { Total length of all visible cracks of that severity } \\
\text { regardless of orientation, as a \% of the principal } \\
\text { dimension of the element, } \\
<20 \%\end{array}$ \\
\hline 3 & Defect occupies $<5 \%$ of surface of the element & ditto $20 \%$ up to $50 \%$ \\
\hline 4 & $\begin{array}{l}\text { Defect occupies } 5 \% \text { up to } 10 \% \text { of the surface of } \\
\text { the element }\end{array}$ & ditto $>50 \%$ up to $100 \%$ \\
\hline 5 & $\begin{array}{l}\text { Defect occupies }>10 \% \text { and up to } 50 \% \text { of the } \\
\text { surface of the element }\end{array}$ & ditto $>100 \%$ up to $200 \%$ \\
\hline 6 & $\begin{array}{l}\text { Defect occupies }>50 \% \text { of the surface of the } \\
\text { element }\end{array}$ & ditto $>200 \%$ \\
\hline
\end{tabular}

\footnotetext{
'The method for scoring the extent of cracks in reinforced concrete is different to that used for masonry and mass concrete.

For severity B, C and D cracks, the extent should be established by adding the crack lengths together, regardless of orientation. This length should be expressed as a \% of the principal dimension of the element as detailed in Tables 7,8 \& 19.

Note all defect extents relate to the visible surface of the element.
}

Table 18 - S/ex codes for reinforced concrete

Figure A.7: Severity and Extent definition for Concrete

\begin{tabular}{|c|c|c|c|c|c|c|}
\hline \multicolumn{7}{|c|}{ CONCRETE } \\
\hline \multirow[b]{2}{*}{ Severity } & \multicolumn{6}{|c|}{ Extent } \\
\hline & 1 & 2 & 3 & 4 & 5 & 6 \\
\hline \multirow{2}{*}{$\begin{array}{l}\text { A } \\
\text { B }\end{array}$} & 0 & & & & & \\
\hline & & 1 & 1.5 & 2 & 2.5 & 3 \\
\hline C & & 1.5 & 2 & 3 & 4.5 & 5.5 \\
\hline \multirow[t]{2}{*}{ D } & & 2 & 3 & 3.5 & 4.5 & 6 \\
\hline & & 3 & 4 & 4.5 & 5 & 7 \\
\hline \multirow{2}{*}{$\mathbf{F}$} & & 5 & 5 & 6 & 7.5 & 9 \\
\hline & & 8 & 8 & 9 & 10 & 10 \\
\hline
\end{tabular}

\begin{tabular}{|l|}
\hline \multicolumn{1}{|c|}{ Defect Level } \\
\hline Minor surface damage \\
\hline $\begin{array}{l}\text { Spalling, small cracks, exposed of } \\
\text { secondary reinforcement }\end{array}$ \\
\hline Exposed of primary reinforcement \\
\hline Permanent structural damage \\
\hline
\end{tabular}

Table A.3: Severity and Extent scores for Concrete 


\begin{tabular}{|c|c|c|}
\hline \multirow[t]{3}{*}{ Severity } & \multicolumn{2}{|c|}{ Definition ${ }^{*}$} \\
\hline & Boards & Other Timbers \\
\hline & See notes $1,2,3,4$ and 5 overleaf & See notes 1 \& 6 to 10 inclusive overleaf \\
\hline A & No visible defects. & No visible defects. \\
\hline B & $\begin{array}{l}\text { Choose most extensive from: - } \\
\text { - Wetness (excluding rainfall) } \\
\text { - Surface softening/Fire damage }<10 \mathrm{~mm} \text {. }\end{array}$ & $\begin{array}{l}\text { Choose most extensive from: - } \\
\text { - Wetness (excluding rainfall) } \\
\text { - Surface softening/Fire damage/Attack } \\
\leq 20 \mathrm{~mm}\end{array}$ \\
\hline $\mathrm{C}$ & Not Used & $\begin{array}{l}\text { Surface softening/Fire damage/Attack } \\
20 \mathrm{~mm} \text { and up to } 100 \mathrm{~mm} \text { deep }\end{array}$ \\
\hline $\mathrm{D}$ & Splits/shakes of any size, with the grain. & $\begin{array}{l}\text { Internal softening (Measured from one face) } \\
<100 \mathrm{~mm} \text { deep. }\end{array}$ \\
\hline $\mathrm{E}$ & $\begin{array}{l}\text { Loose/damaged/badly corroded/missing } \\
\text { fastenings, i.e. bolts, screws, etc. }\end{array}$ & $\begin{array}{l}\text { Internal softening (Measured from one face) } \\
100 \mathrm{~mm} \text { deep and greater. }\end{array}$ \\
\hline $\mathrm{F}$ & $\begin{array}{l}\text { Surface softening/Fire damage } 10 \mathrm{~mm} \text { up to } \\
35 \mathrm{~mm} \text {. }\end{array}$ & $\begin{array}{l}\text { Splitting/crushing/distortion but not at splice or } \\
\text { end of timber. }\end{array}$ \\
\hline G & Not Used & $\begin{array}{l}\text { Splitting/crushing/distortion at splice or end of } \\
\text { timber. }\end{array}$ \\
\hline $\mathrm{H}$ & $\begin{array}{l}\text { Choose most extensive from: - } \\
\text { - Surface softening/Fire damage/loss of } \\
\text { element section }>35 \mathrm{~mm} \text {. } \\
\text { - Total loss of timber section } \\
\text { - Crushing/distortion/splits across grain } \\
\text { - Permanent displacement }\end{array}$ & $\begin{array}{l}\text { Choose most extensive from: - } \\
\text { - Loose/damaged/badly corroded/missing } \\
\text { structural connections } \\
\text { - Substantial/total loss of timber section } \\
\text { Permanent deformation/displacement of } \\
\text { timber. }\end{array}$ \\
\hline
\end{tabular}

\begin{tabular}{|c|l|}
\hline Extent & \multicolumn{1}{|c|}{ Definition } \\
\hline Note & Surface includes all visible surfaces of the element \\
\hline I & No visible defects \\
\hline 2 & Localised defect due to local circumstances (e.g. local impact damage) \\
\hline 3 & Defect occupies < $5 \%$ of surface ${ }^{*}$ of the element \\
\hline 4 & Defect occupies $5 \%$ up to $10 \%$ of the surface ${ }^{*}$ of the element. \\
\hline 5 & Defect occupies $>10 \%$ up to $50 \%$ of the surface ${ }^{*}$ of the element. \\
\hline 6 & Defect occupies $>50 \%$ of the surface ${ }^{*}$ of the element \\
\hline
\end{tabular}

* For certain defects, the extent is not always expressed in terms of the surface of the element. See notes I to 10 in section 9.7.2.

It is essential that table 20 is read in conjunction with the notes in section 9.7.2

Table 20 - S/ex codes for timber

Figure A.8: Severity and Extent definition for Timber

\begin{tabular}{|c|c|c|c|c|c|c|}
\hline \multicolumn{7}{|c|}{ TIMBER } \\
\hline & \multicolumn{6}{|c|}{ Extent } \\
\hline Severity & 1 & 2 & 3 & 4 & 5 & 6 \\
\hline \multirow{2}{*}{ A } & 0 & & & & & \\
\hline & & 1 & 1.5 & 2 & 3 & 4 \\
\hline C & & 2 & 2.5 & 3.5 & 5 & 7 \\
\hline D & & 3 & 3.5 & 4 & 5.5 & 7.5 \\
\hline E & & 4 & 4.5 & 5.5 & 7.5 & 9 \\
\hline $\mathbf{F}$ & & 5 & 5.5 & 6 & 7 & 8 \\
\hline G & & 6 & 6.5 & 7 & 8 & 9 \\
\hline $\mathbf{H}$ & & 7 & 8 & 9 & 10 & 10 \\
\hline
\end{tabular}

\begin{tabular}{|l|}
\hline \multicolumn{1}{|c|}{ Defect Level } \\
\hline Minor defects \\
\hline Surface softening, fire damage, splits \\
\hline Surface and internal softening, \\
fire damage, crushing, loss of timber \\
section \\
\hline Permanent structural damage \\
\hline
\end{tabular}

Table A.4: Severity and Extent scores for Timber 


\begin{tabular}{|c|c|}
\hline Severity & Definition \\
\hline A & No visible defects to masonry. (Cracks are scored separately). \\
\hline B & \begin{tabular}{l}
\multicolumn{2}{c}{ Choose most extensive from:- } \\
- Spalling / softness of brick up $\quad \begin{array}{l}\text { Indications of } \\
\text { wetness/presence of water } \\
\text { to } 10 \mathrm{~mm} .\end{array}$ \\
$\begin{array}{ll}\text { (define as percolation, run } \\
\text { - Spalling / softness of stone and } \\
\text { mass concrete up to } 20 \mathrm{~mm} .\end{array} \quad \begin{array}{l}\text { down etc) excluding exposure } \\
\text { to rainfall. }\end{array}$
\end{tabular} \\
\hline $\mathrm{C}$ & Deterioration of pointing (note maximum and typical depth lost). \\
\hline $\mathrm{D}$ & $\begin{array}{l}\text { - Spalling / softness of brick }>10 \mathrm{~mm} \text { and up to } 100 \mathrm{~mm} \text { thickness } \\
\text { - Spalling / softness of stone and mass concrete }>20 \mathrm{~mm} \text { and up to } \\
200 \mathrm{~mm} \text { thickness }\end{array}$ \\
\hline $\mathrm{E}$ & Hollowness/Drumming (not associated with B or D) \\
\hline Ex & $\begin{array}{l}\text { Includes all incidences of:- } \\
\text { Loose / missing masonry and wedged bricks. }\end{array}$ \\
\hline $\mathrm{F}$ & $\begin{array}{l}\text { Choose most extensive from:- } \\
\text { - Bulging/Distortion } \bullet \text { Displacement } \\
\text { - Out of plumb }\end{array}$ \\
\hline
\end{tabular}

\begin{tabular}{|c|l|}
\hline Extent & \multicolumn{1}{c|}{ Definition } \\
\hline I & No visible defects to masonry. (Cracks are scored separately). \\
\hline 2 & $\begin{array}{l}\text { Localised defect due to local circumstances (such as localised } \\
\text { mechanical damage caused by a single bridge strike). }\end{array}$ \\
\hline 3 & Defect occupies $<5 \%$ of surface of the element. \\
\hline 4 & Defect occupies $5 \%$ up to $10 \%$ of the surface of the element. \\
\hline 5 & Defect occupies $>10 \%$ and up to $50 \%$ of the surface of the element. \\
\hline 6 & Defect occupies $>50 \%$ of the surface of the element. \\
\hline
\end{tabular}

Note all defect extents relate to the visible surface of the element.

Table 16 - S/ex codes for masonry

Figure A.9: Severity and Extent definition for Masonry

\begin{tabular}{|c|c|c|c|c|c|c|}
\hline \multicolumn{7}{|c|}{ MASONRY } \\
\hline \multirow[b]{2}{*}{ Severity } & \multicolumn{6}{|c|}{ Extent } \\
\hline & 1 & 2 & 3 & 4 & 5 & 6 \\
\hline \multirow{2}{*}{$\begin{array}{l}\text { A } \\
\text { B }\end{array}$} & 0 & & & & & \\
\hline & & 1 & 1 & 1.2 & 1.5 & 2 \\
\hline C & & 1.5 & 1.5 & 2 & 2.5 & 3 \\
\hline \multirow{2}{*}{ D } & & 3 & 3 & 3.5 & 5 & 6 \\
\hline & & 3 & 4 & 5 & 6.5 & 8 \\
\hline $\begin{array}{l}\mathbf{E} \\
\mathbf{F}\end{array}$ & & 3 & 4.5 & 6 & 8 & 10 \\
\hline
\end{tabular}

\begin{tabular}{|l|}
\hline \multicolumn{1}{|c|}{ Defect Level } \\
\hline Minor defects \\
\hline $\begin{array}{l}\text { Spalling, pointing degradation, } \\
\text { water ingress }\end{array}$ \\
\hline Spalling, hollowness, drumming \\
\hline $\begin{array}{l}\text { Missing masonry, permanent } \\
\text { distortion }\end{array}$ \\
\hline
\end{tabular}

Table A.5: Severity and Extent scores for Masonry 


\section{A-4 Component degradation analysis}

\section{i. Metal Main Girder}

There are a total of more than 37,000 bridge main girders component in the whole bridge population and around $80 \%$ of them are in the good and poor condition (Figure A.10). Since the number of the data contains historical work done are quite low, there are only 604 sets of girders that were actually studied in the analysis. This means that only $1.6 \%$ of the population were analysed. Figure A.11(a) shows the distribution of all types of work that were recorded in the database. Although there are a significant number of records on minor and major intervention, there are only 4 entries were recorded for the renewal of bridge main girders.

Components in the same condition state may establish different type of defects which would require a specific repair work. It is useful to know in each these work categories (minor, major repair, replacement, servicing), what type of specific work are often carried out. This can be seen in Figure A.11(b), (c), where the distributions of the specific works for minor and major repair categories are shown.

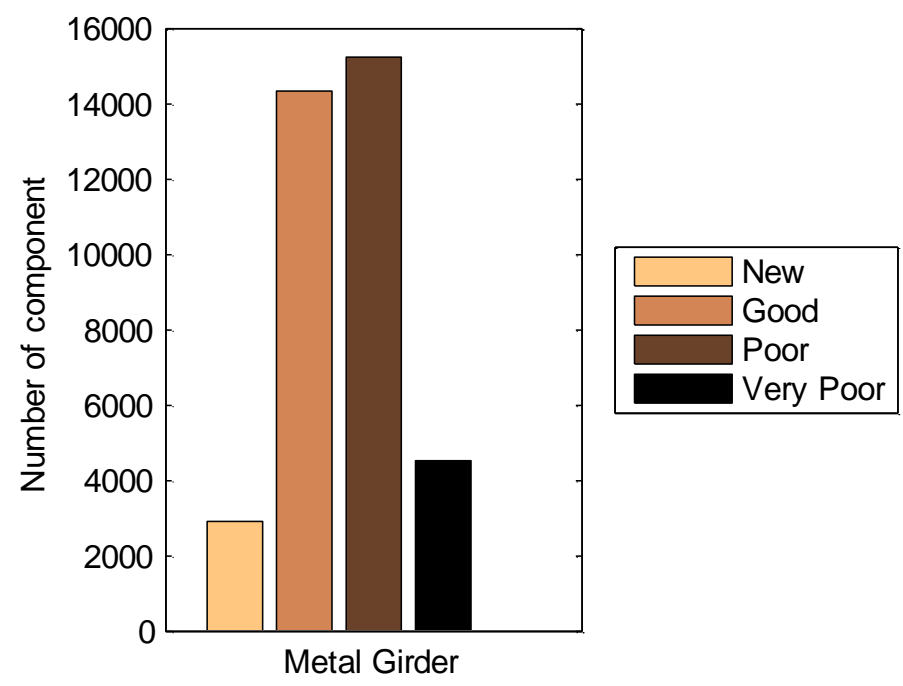

Figure A.10: Initial condition distribution of metal main girders 
(a) All types of records

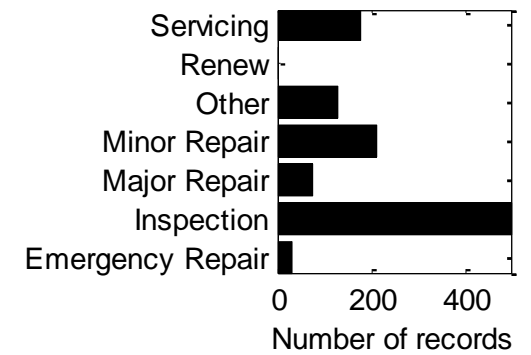

(c) Major Repair

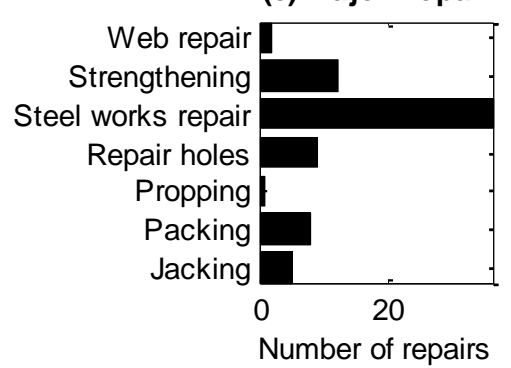

(b) Minor Repair

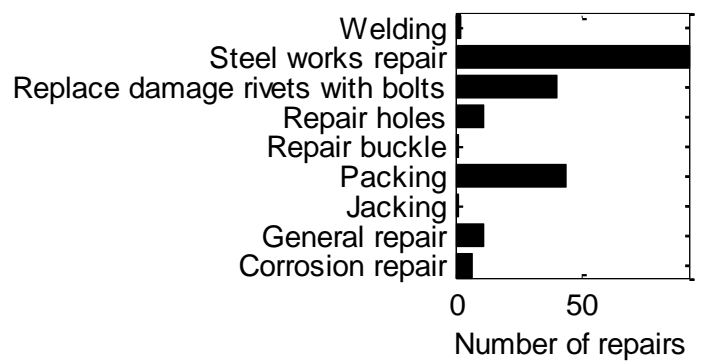

(d) Servicing

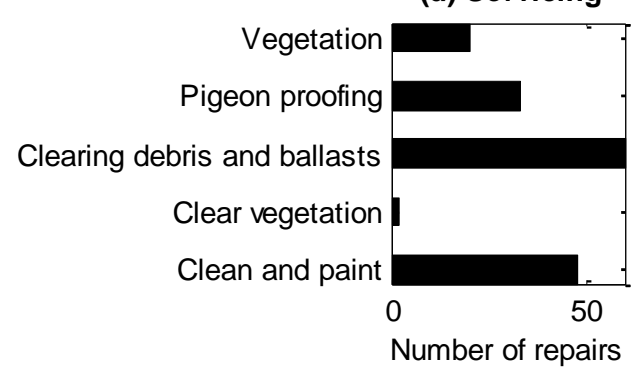

Figure A.11: Distributions of specific works for Metal Girder

\section{Distribution fitting}

Figure A.12 shows the probability plot when fitting a Weibull distribution to the data. The data are the lifetimes of a set of two girders reaching a state where minor repair is required from the 'as new' state. The best-fitted straight line shows a very good fit with the correlation very close to 1 . This suggests that the Weibull distribution is a very good fit to the data. The distribution parameters are shown to the right of the graph, the shape parameter illustrates that the rate of deteriorating from the new to the good condition for main girders is increasing with time (wear-out characteristics). Figure A.14 shows that the rate of reaching the good condition from the 'as new' condition is double after 20 years. However the rate of reaching the poor condition shows unexpected behaviour, it is suspected that the lack of data has resulted in the decreasing rate of failure with time (Figure A.13). Even though there were 4 records of the main girder replacements, inspection information was missing which prevents the derivation of the lifetime data. Thus, a distribution could not be fitted to model the rate of main girders replacement. The estimation method was employed to estimate the rate of girder replacement. 


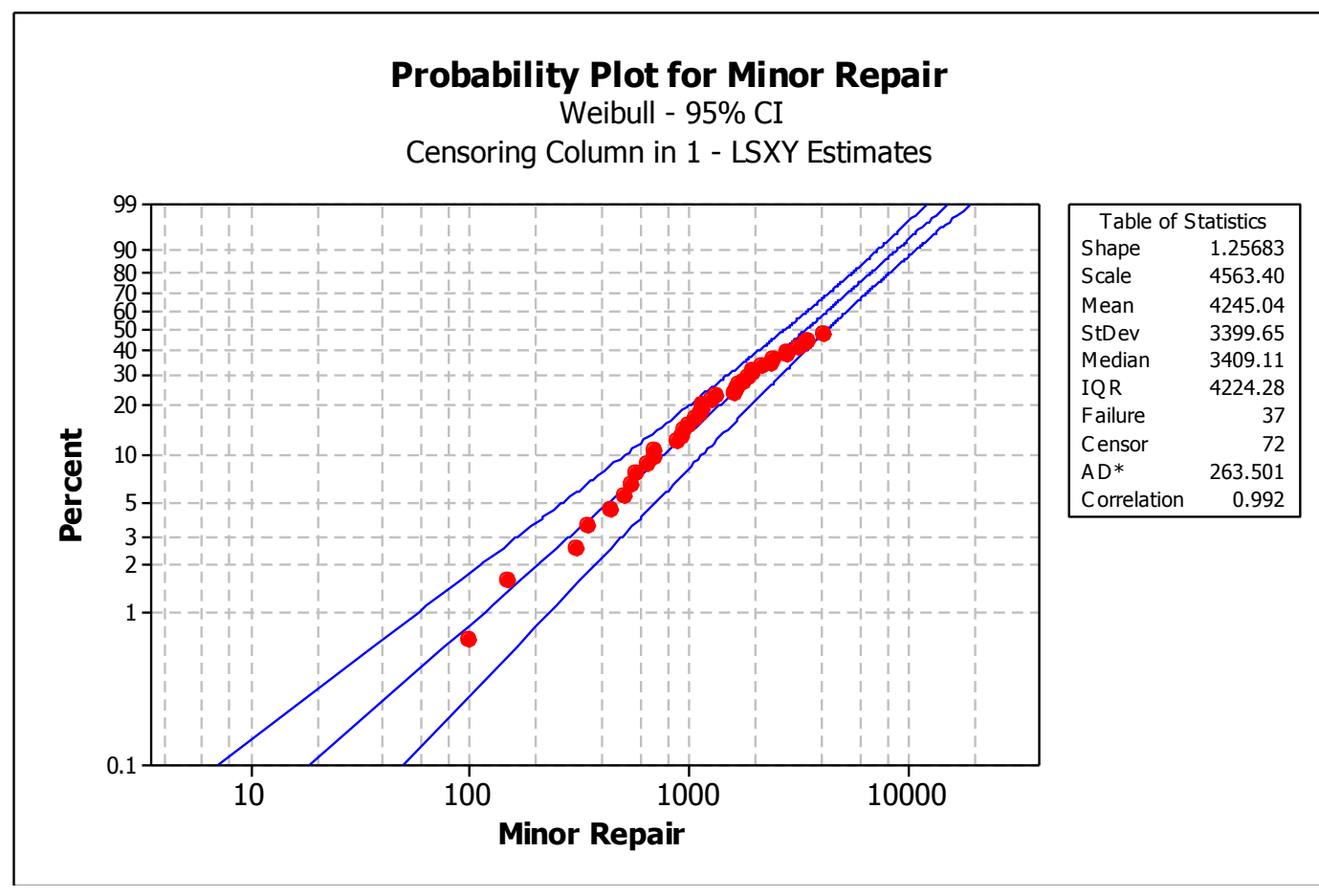

Figure A.12: Probability plot of the time to the point when Minor repair is needed

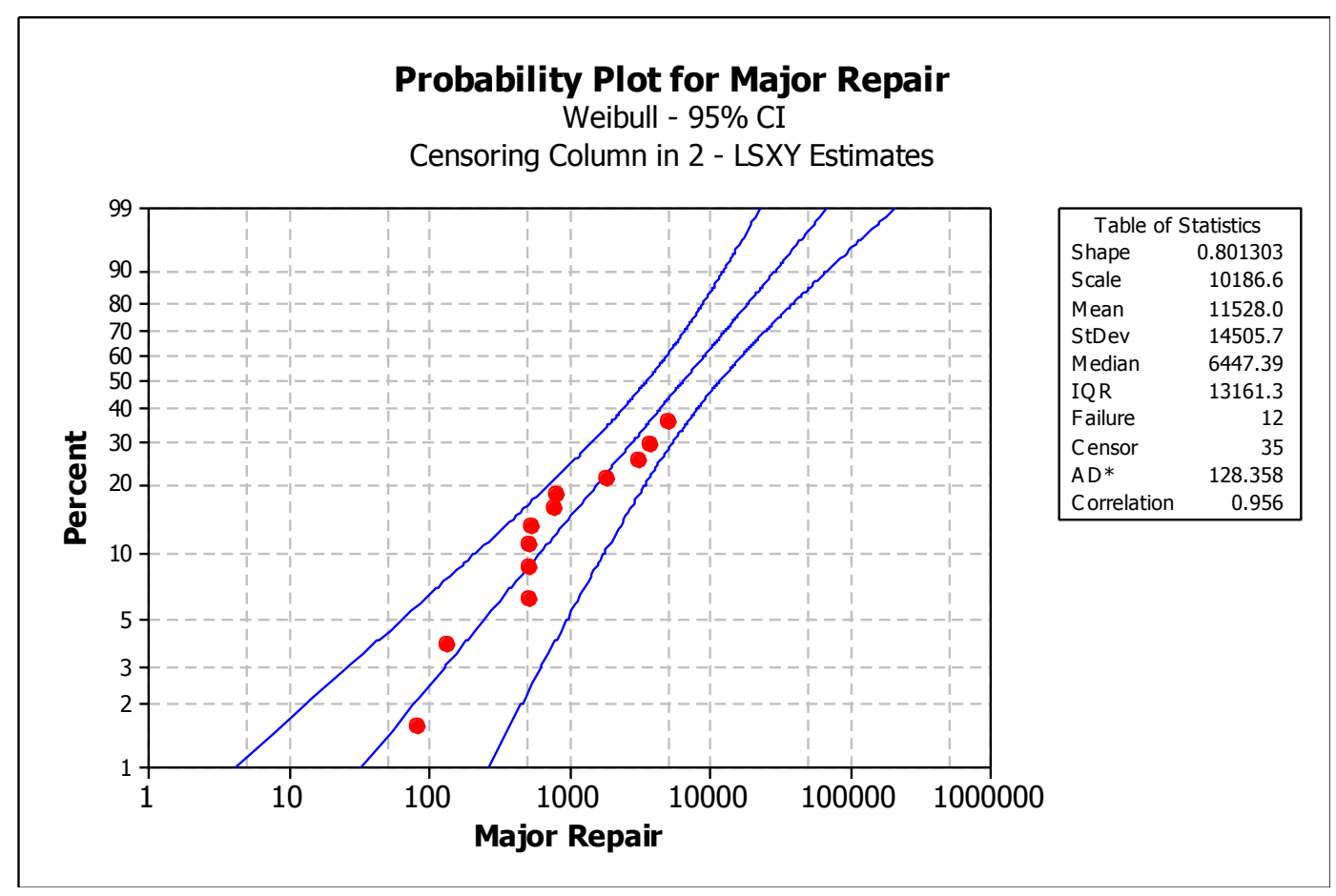

Figure A.13: Probability plot of the time to the point when Major repair is needed 


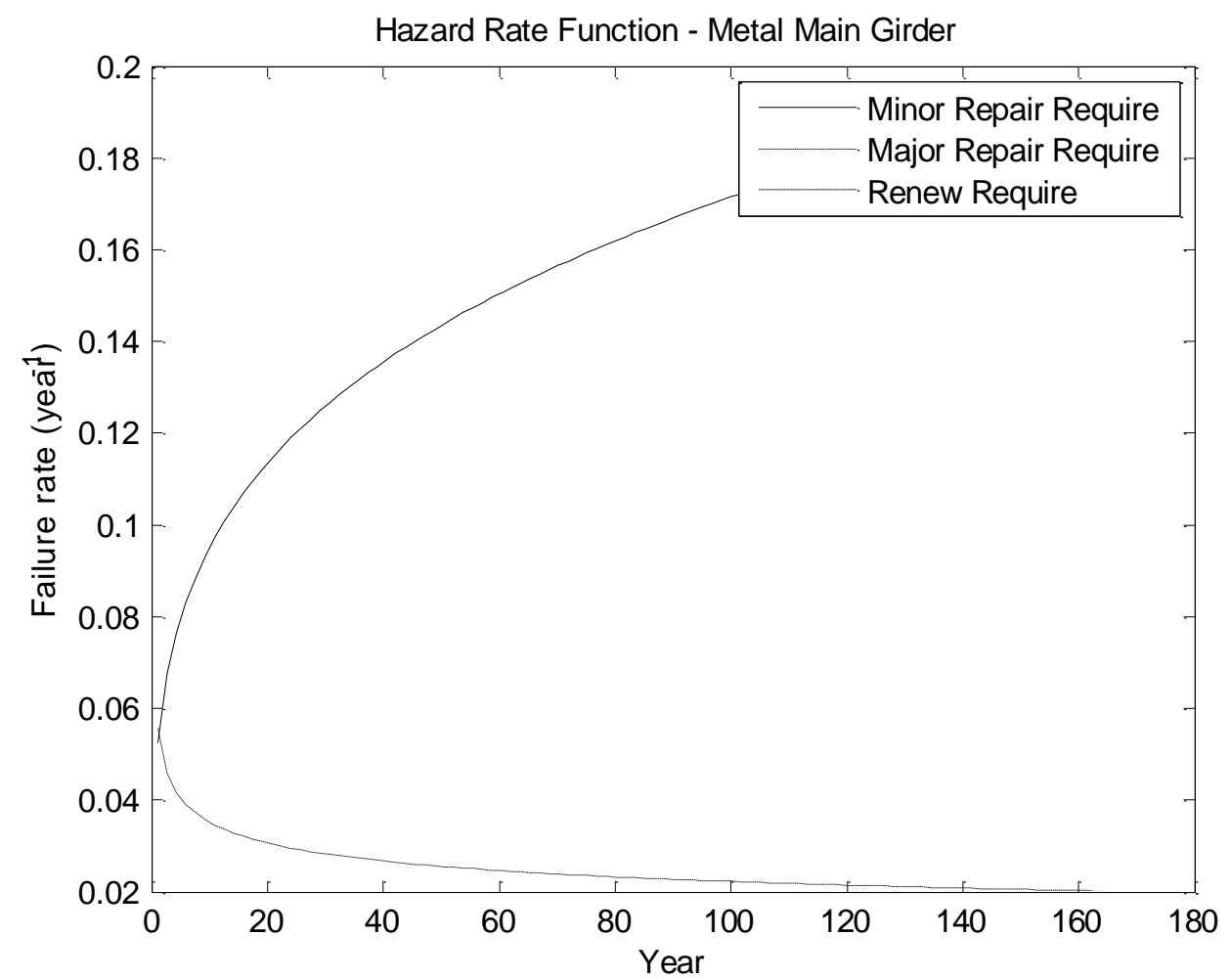

Figure A.14: Hazard rate function which shows the rates that an intervention is needed at different life-time 


\section{ii. Bridge Deck}

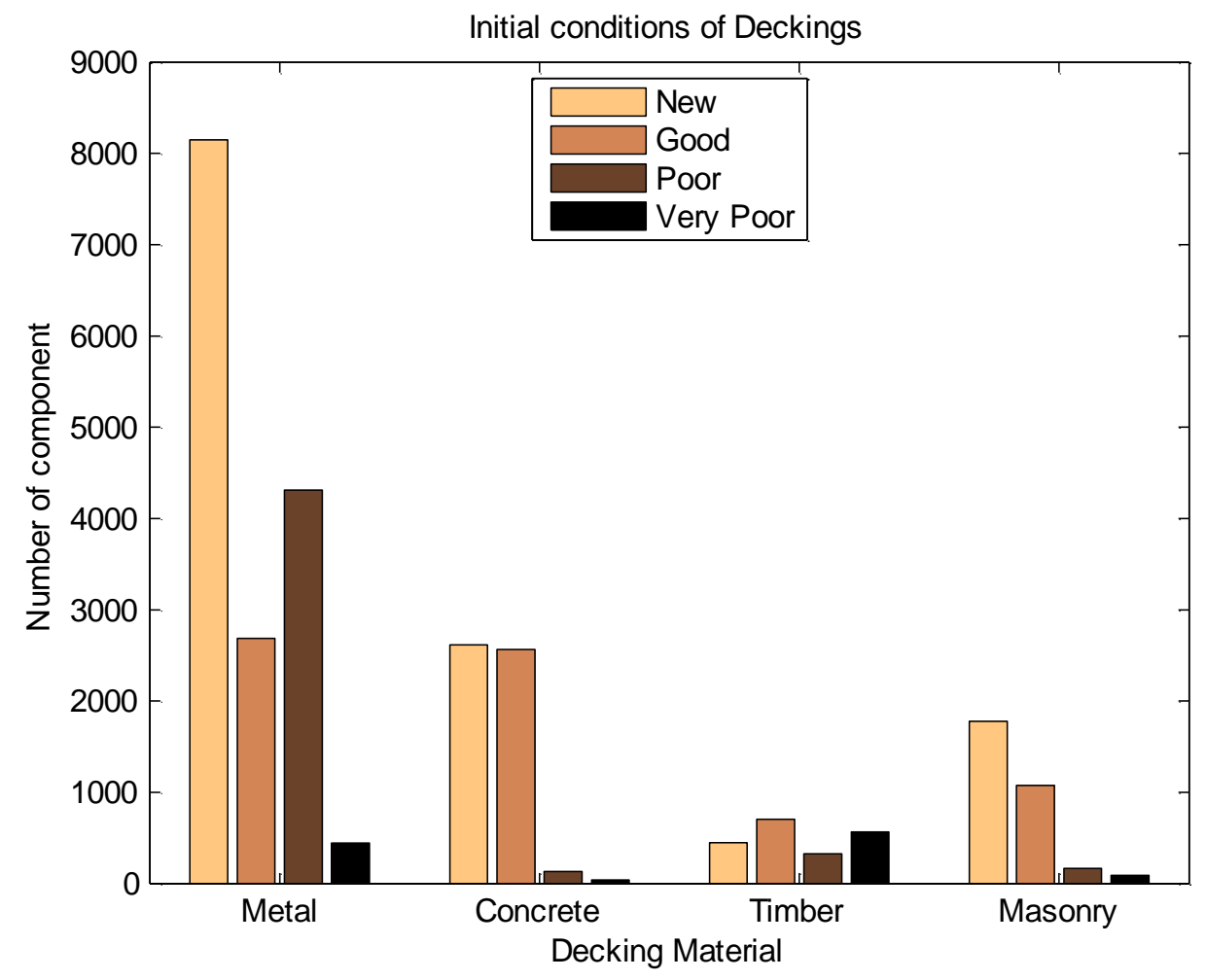

Figure A.15: Initial condition distribution of Deckings

There are four different types of bridge deckings in metal underbridges. Metal is the most popular decking material with 15,589 metal decks with almost three times more than the population of concrete deckings, seven times more than timber decks and five times more than decks made of masonry. The current condition distribution varies according to different bridge deck materials. Almost the entire population of concrete decks are in the new and good condition with only about $1 \%$ of the population is in the very poor condition that would need replacement. Metal deck shows a different distribution with over $50 \%$ of the population is in new condition, $17 \%$ would require minor intervention and almost $30 \%$ require major repair. High deterioration rate combining with the fact that timber deck was once a popular choice of decking materials shows that the population of timber decks are quite evenly spread. That is almost a quarter of the populations are in each condition band. Masonry decks even though are listed here however this deck type will not be discussed because there were not enough failure data available to support the study. 


\section{iii. Metal Deck}
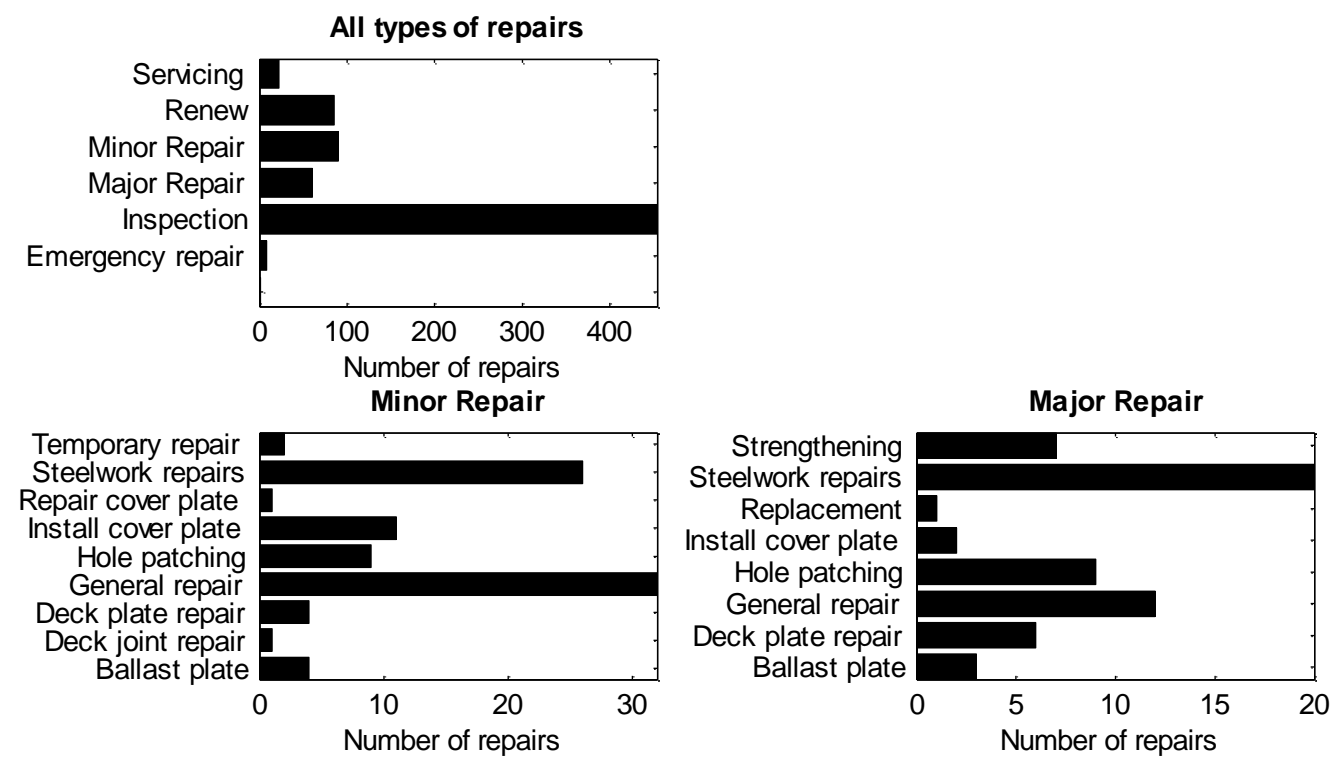

Figure A.16: Distributions of specific works for Metal Deck

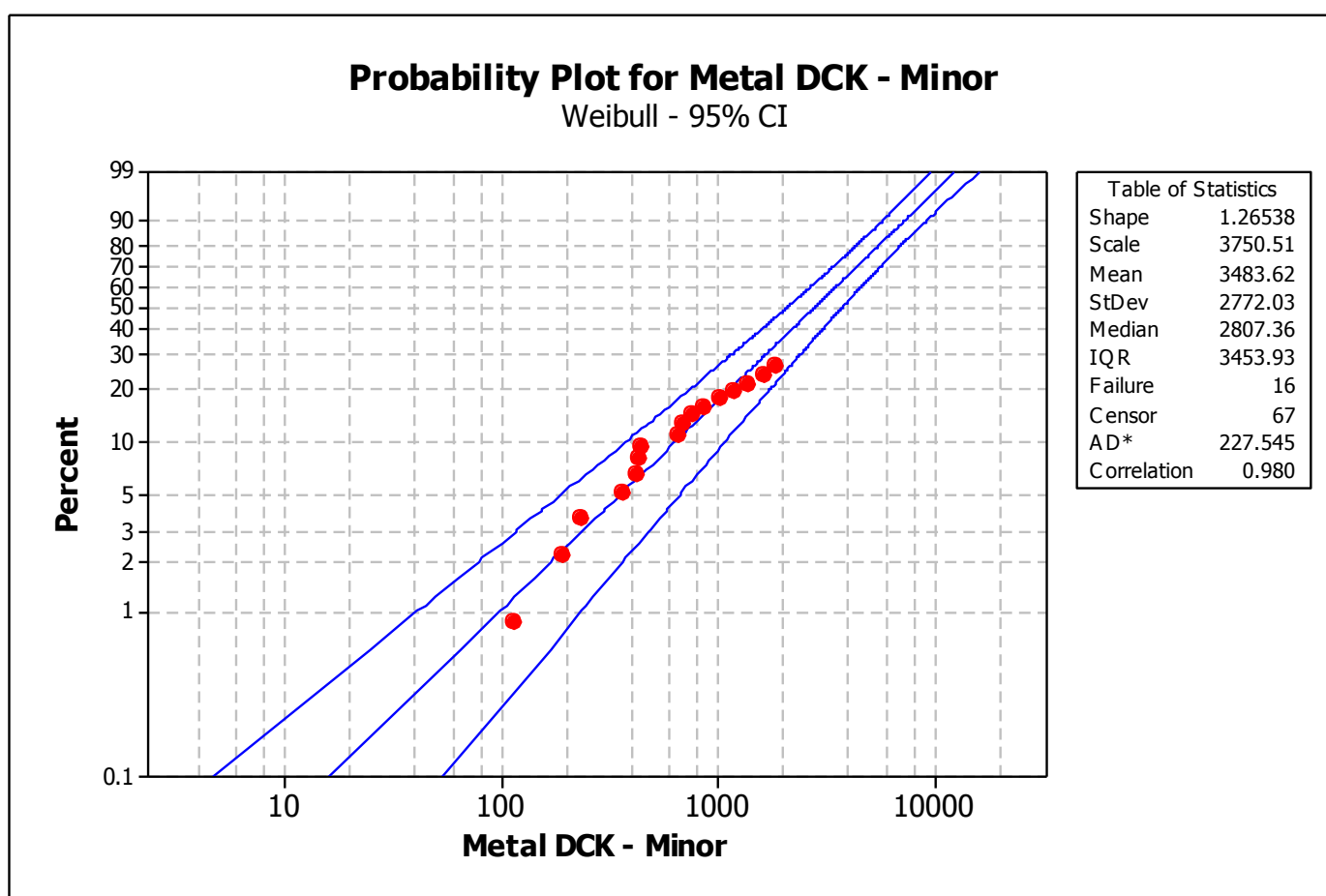

Figure A.17: Probability plot of the time to the point when Minor repair is needed 


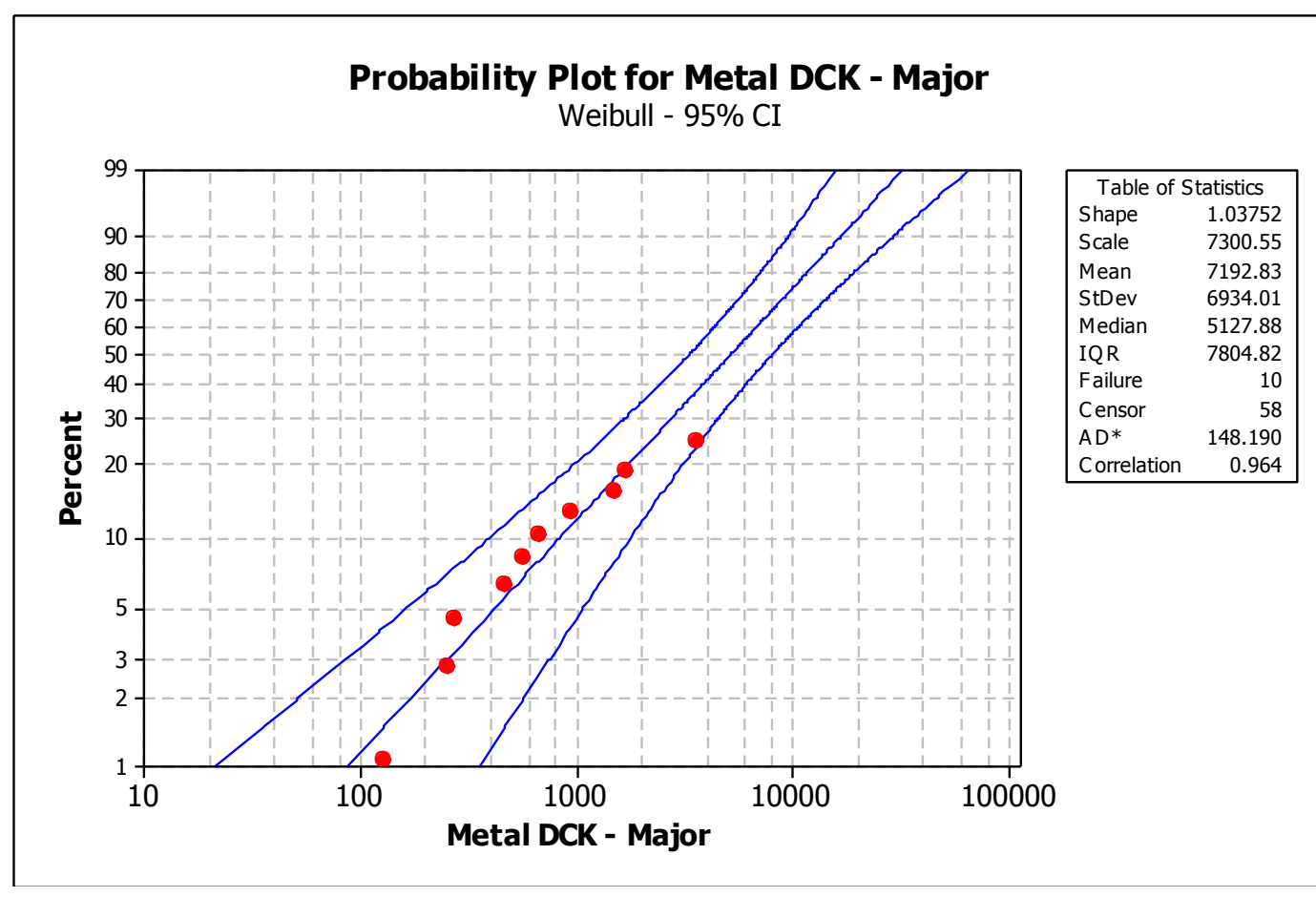

Figure A.18: Probability plot of the time to the point when Major repair is needed

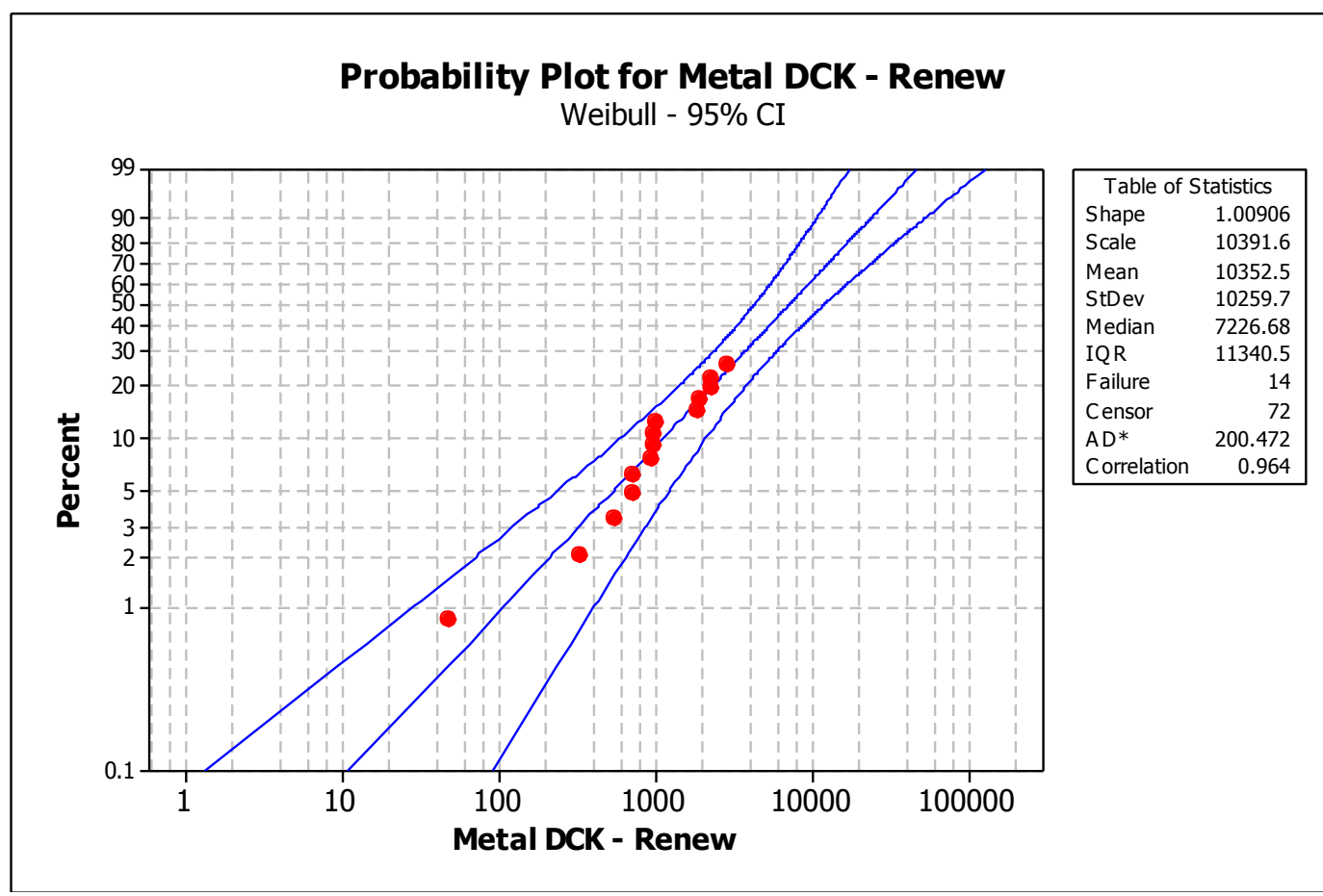

Figure A.19: Probability plot of the time to the point when Renewal is needed 


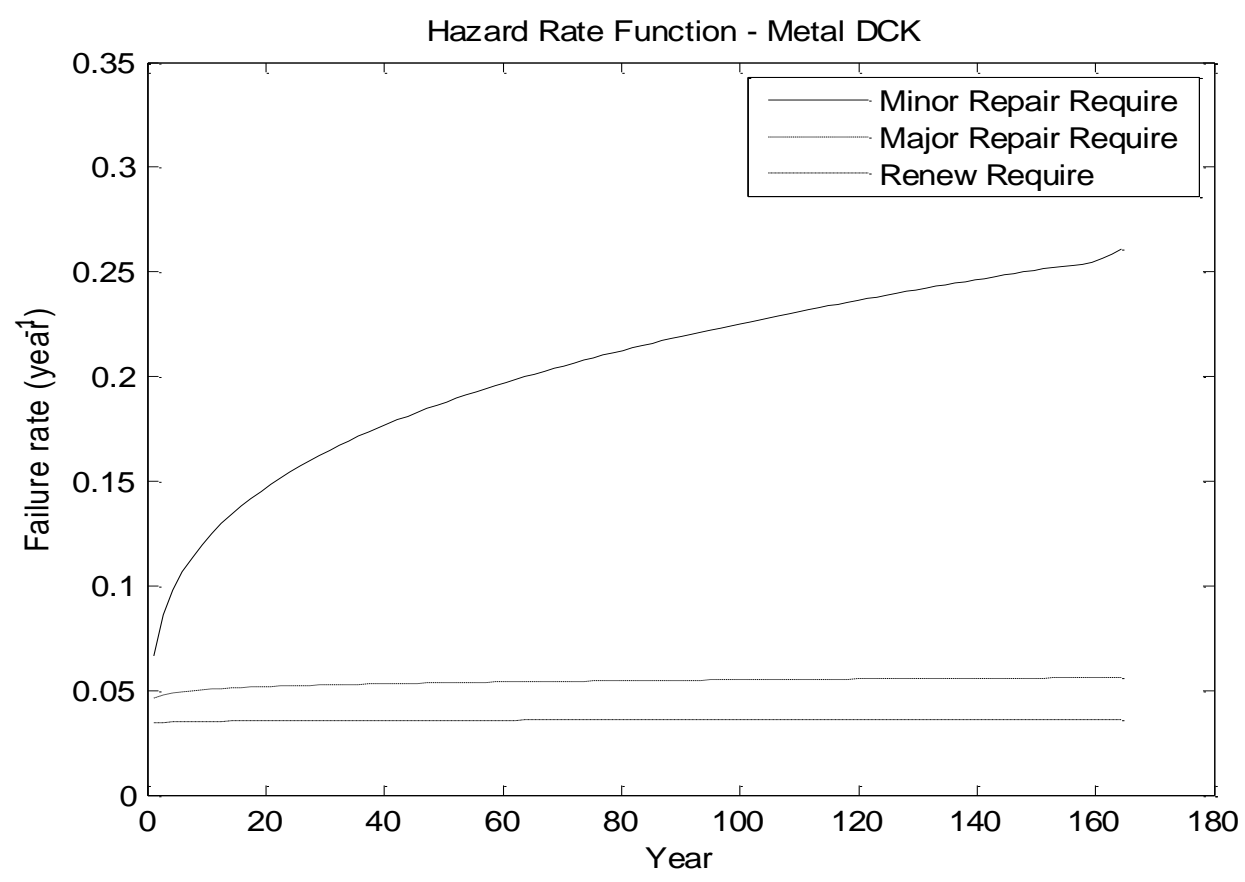

Figure A.20: Hazard rate function which shows the rates that an intervention is needed at different life-time

For metal decks, the shape parameters obtains for a component reaching a poor and a very poor state are very close to 1 , this means that the rate of a component requires major repairs and replacement is constant over time and is about 0.05 and 0.03 metal deck per year (Figure A.20). In contrast, the rate of metal decks moving from a new condition to a good condition is increasing from 0.06 metal decks per year to about 0.18 after 60 years. Thus it is three times more likely to require a minor repair for a deck in 60 years old comparing with the new metal deck. 


\section{iv. Concrete Deck}

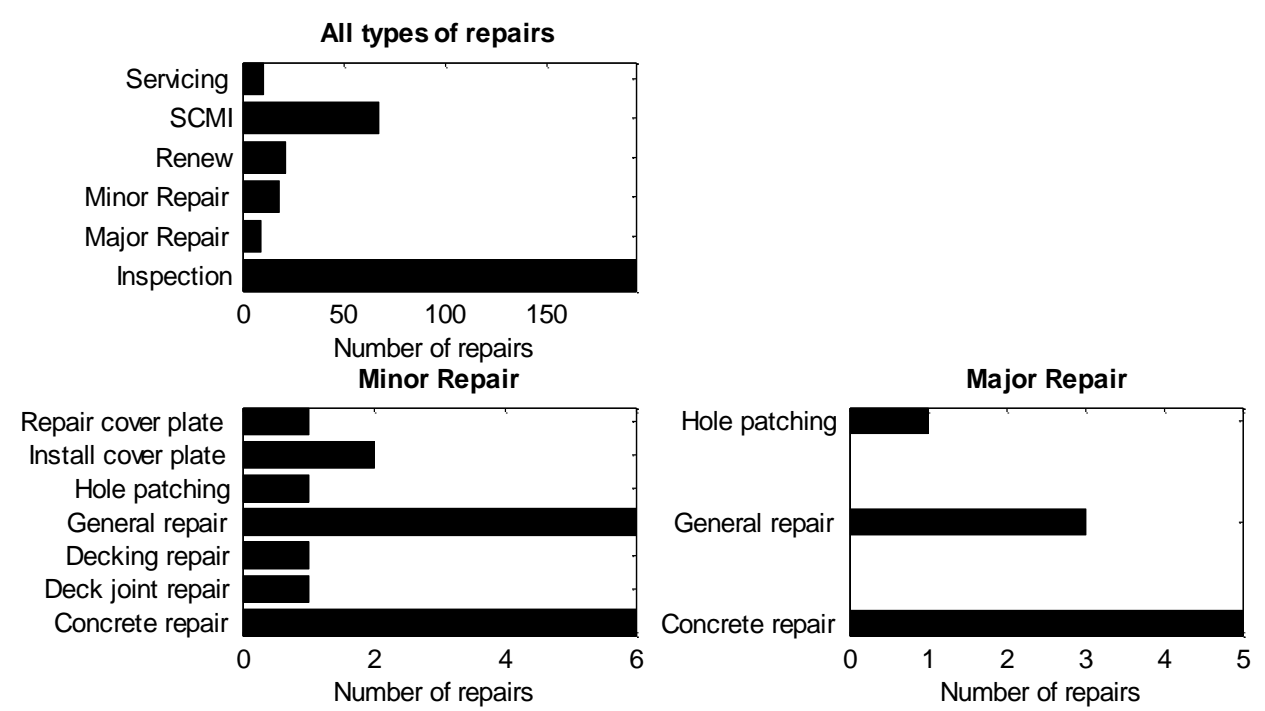

Figure A.21: Distributions of specific works for Concrete Deck

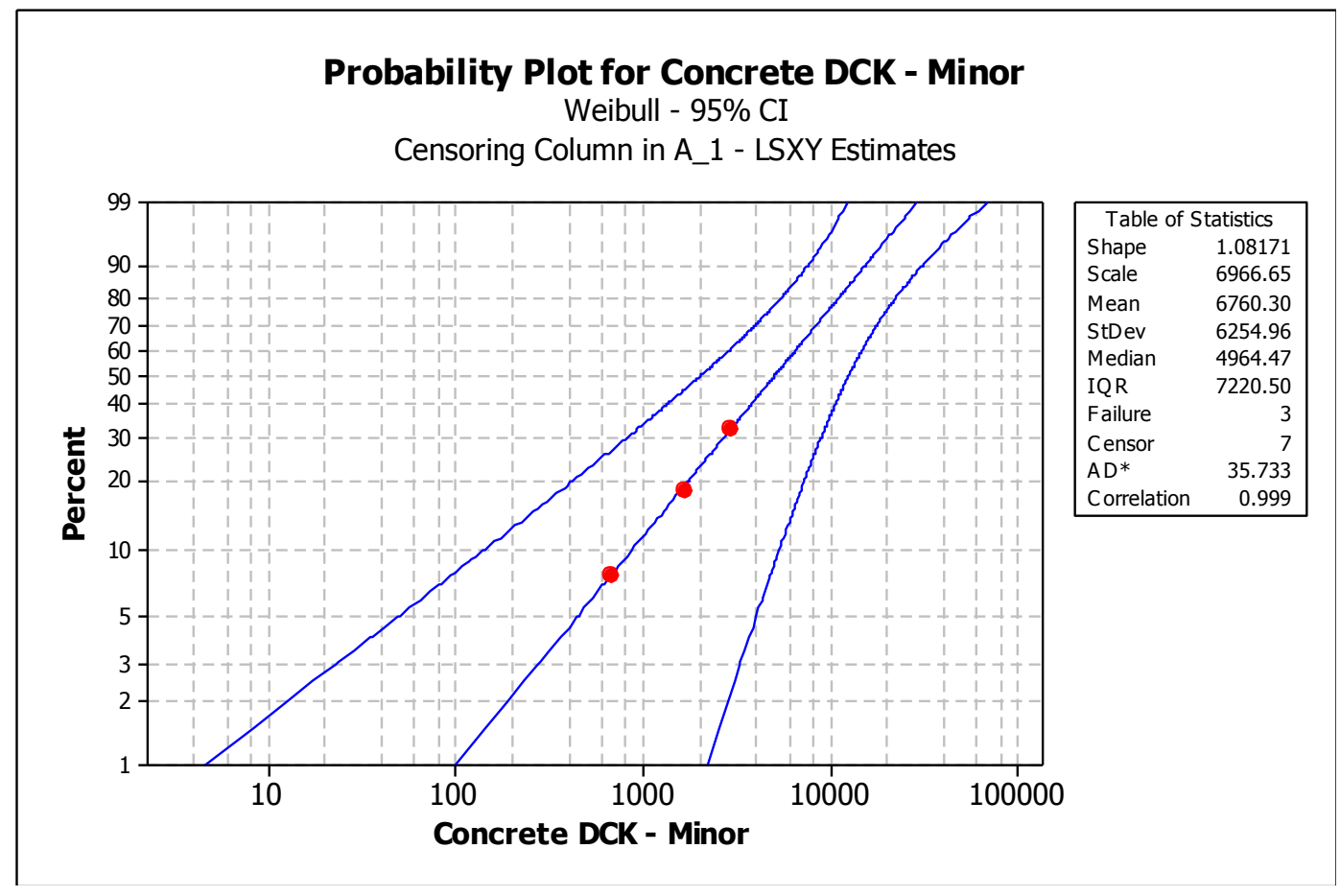

Figure A.22: Probability plot of the time to the point when Minor repair is needed 


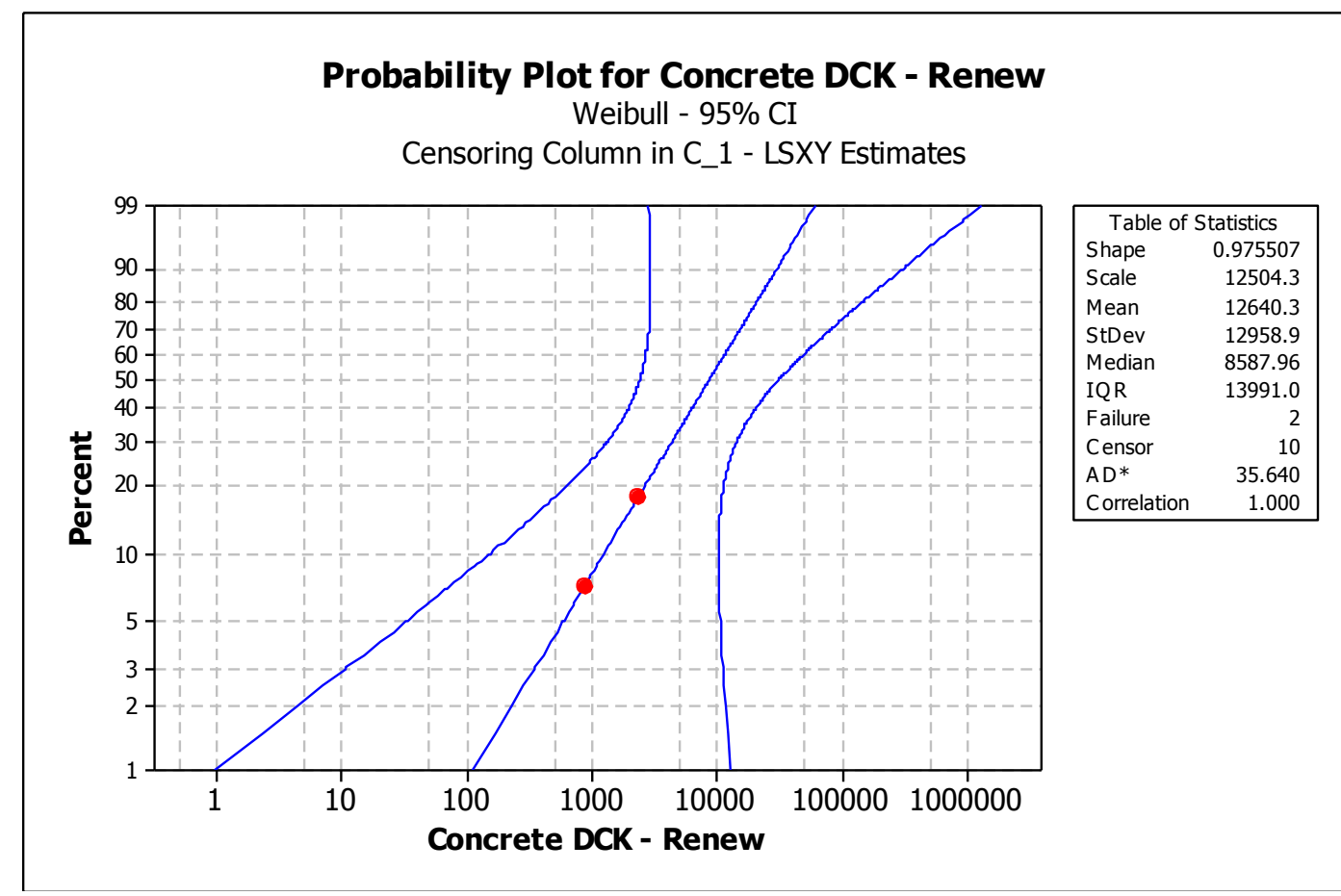

Figure A.23: Probability plot of the time to the point when Renewal is needed

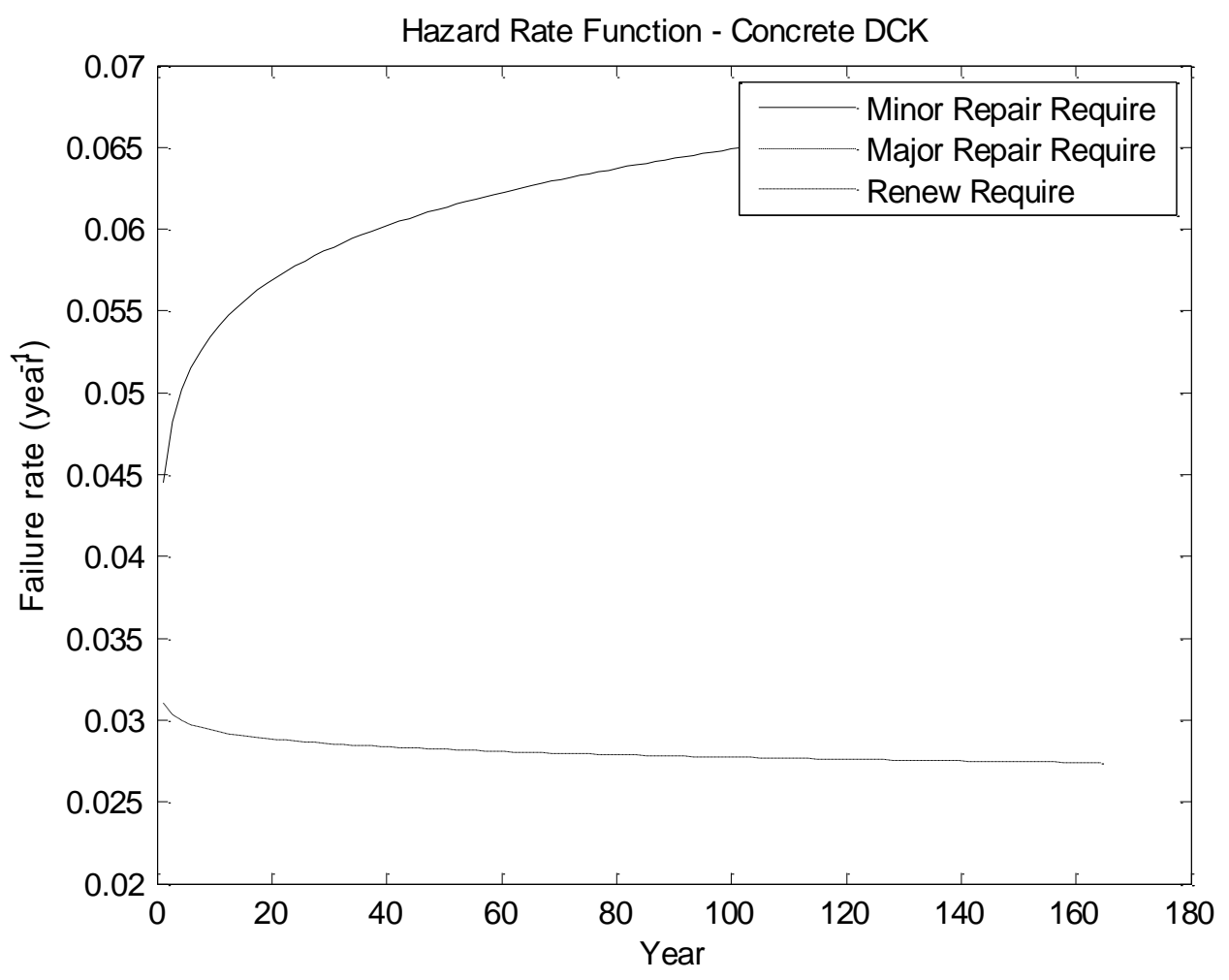

Figure A.24: Hazard rate function which shows the rates that an intervention is needed at different life-time

As demonstrated in Figure A.21, the majority (>95\%) of the concrete deck population are in new and good conditions. This, combining with the young age of the population, has resulted in 
less number of repairs recorded for bridge concrete decks. There are only 10 minor repairs, 4 major repairs and 12 deck replacements. Therefore the confident intervals that can be seen on the results are very large.

\section{v. Timber Deck}

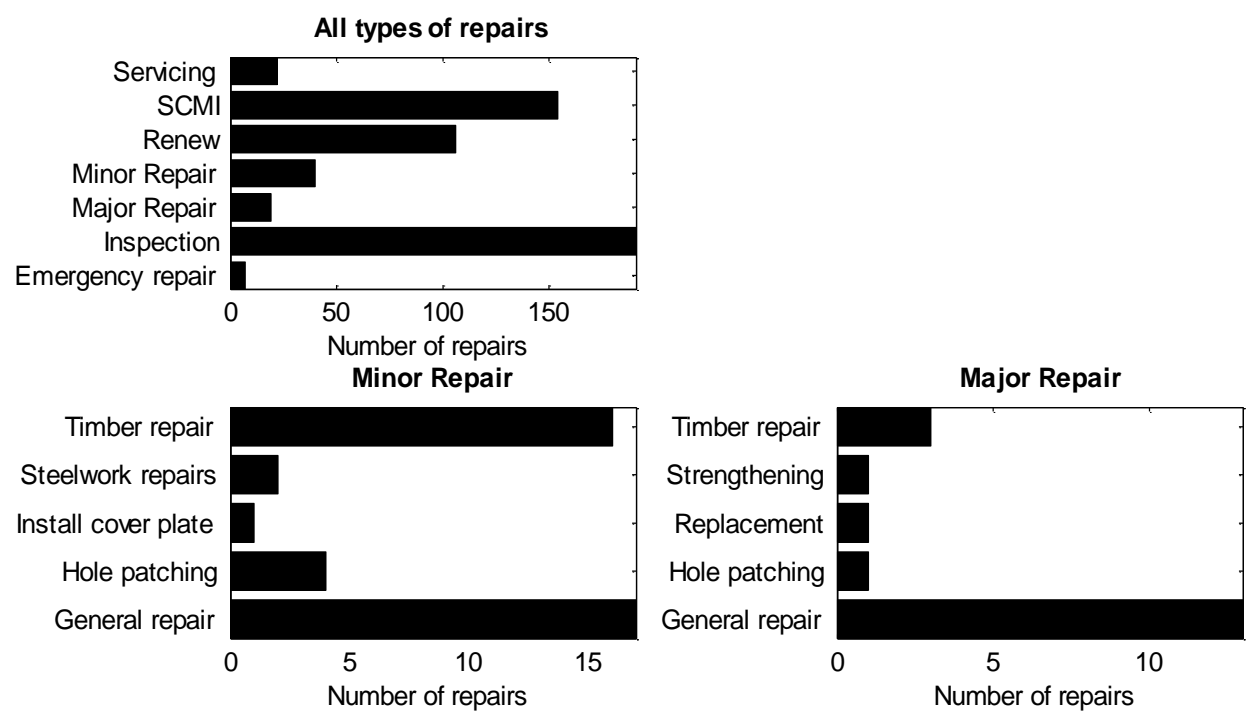

Figure A.25: Distributions of specific works for Timber Deck

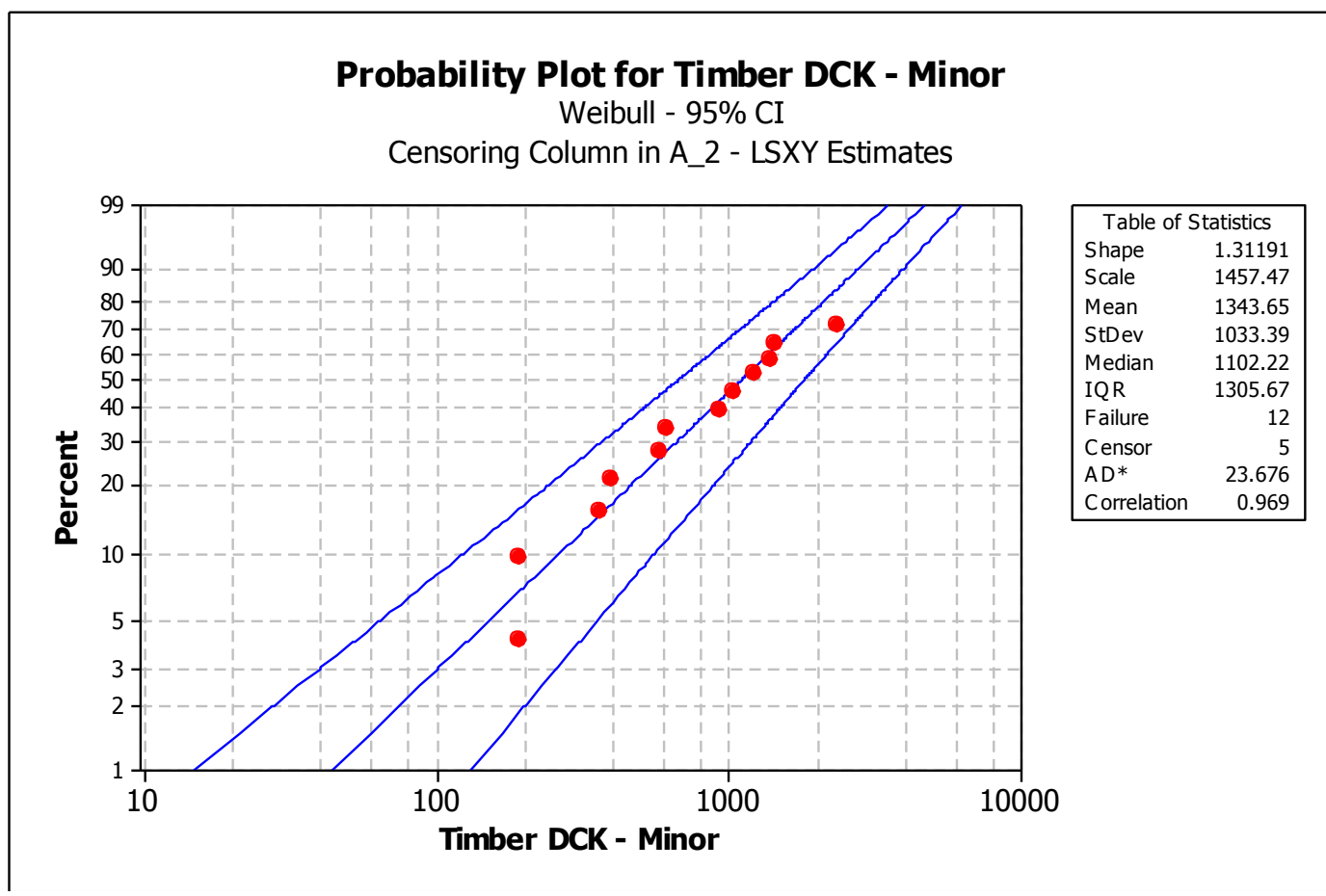

Figure A.26: Probability plot of the time to the point when Minor repair is needed 


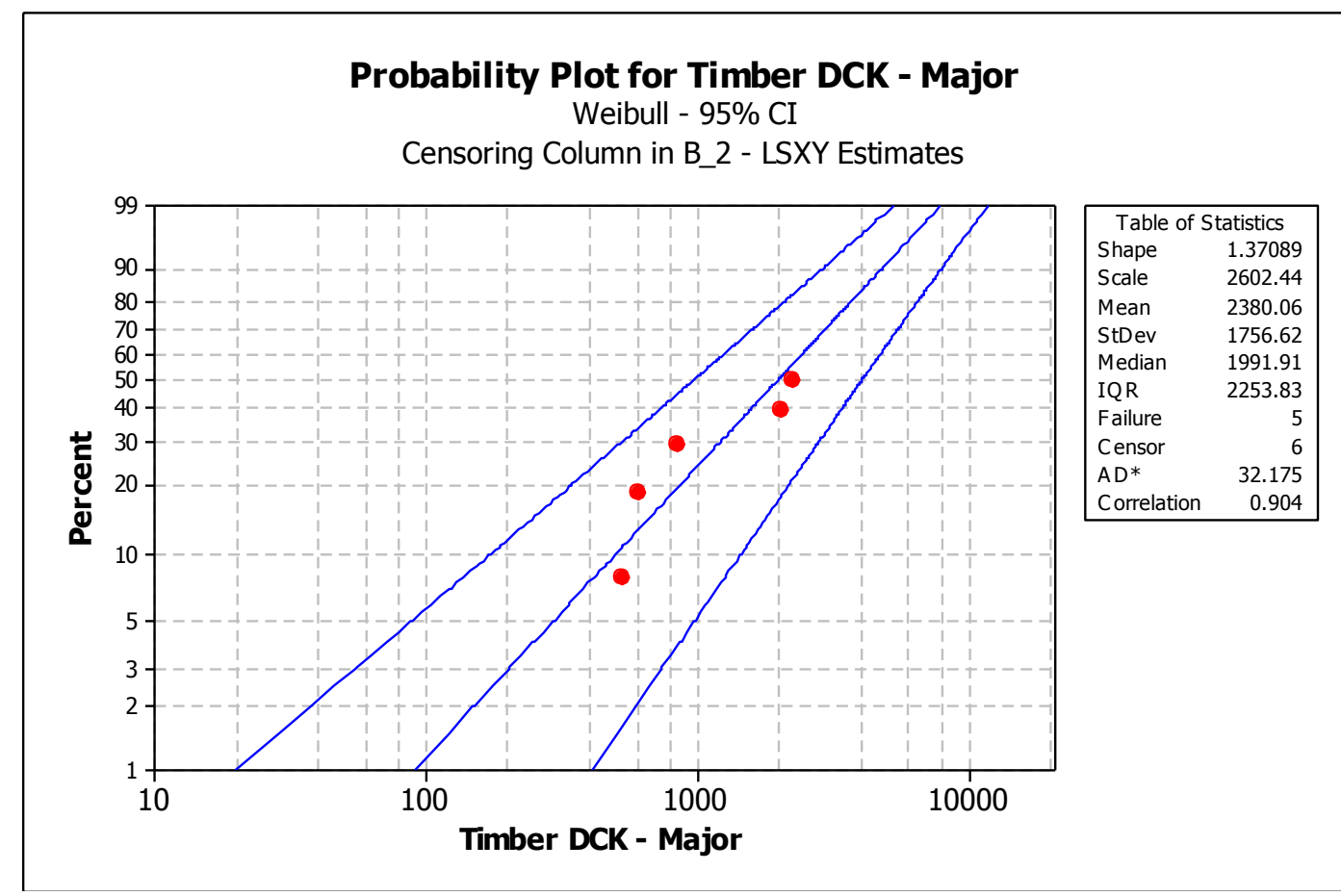

Figure A.27: Probability plot of the time to the point when Major repair is needed

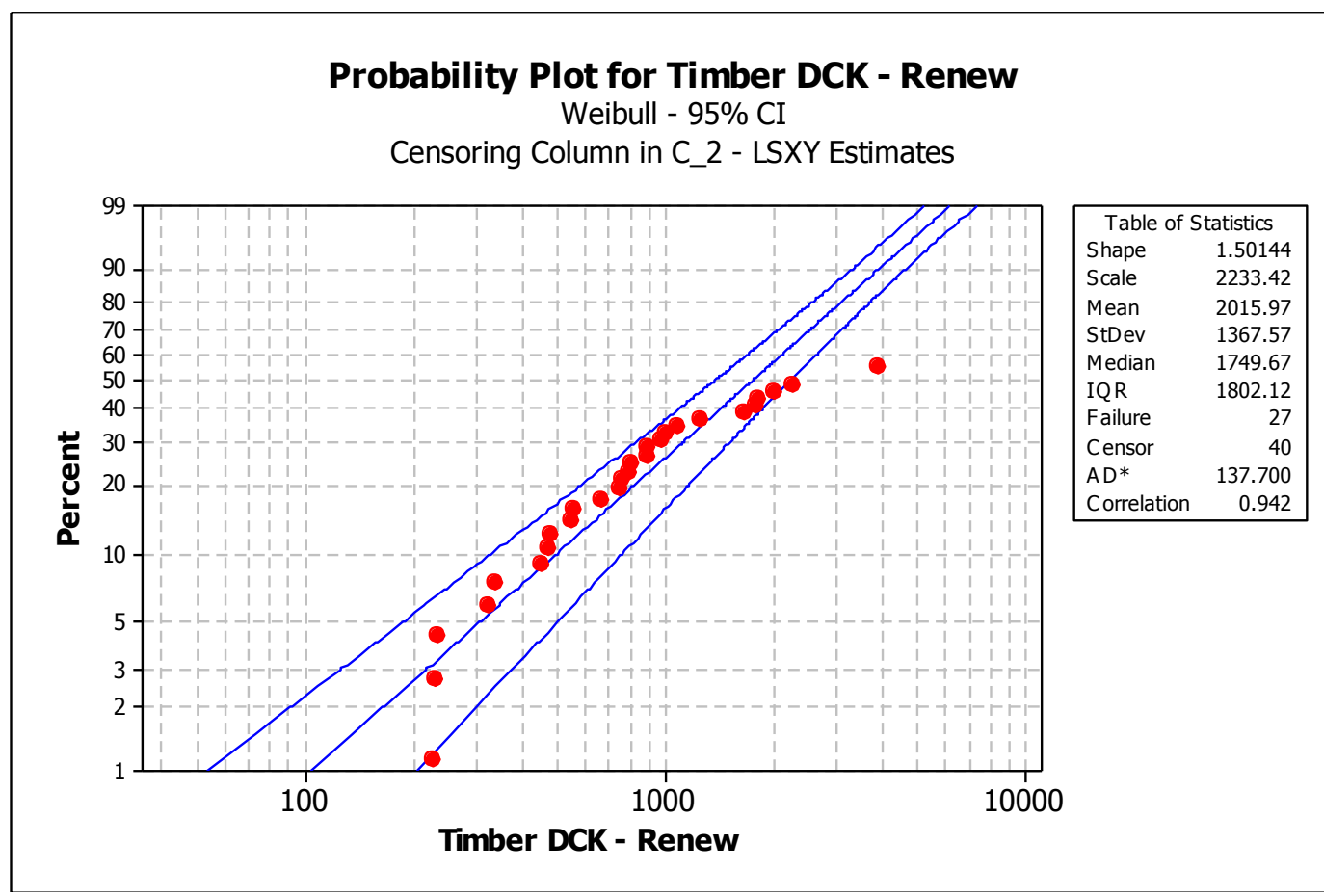

Figure A.28: Probability plot of the time to the point when Renewal is needed 


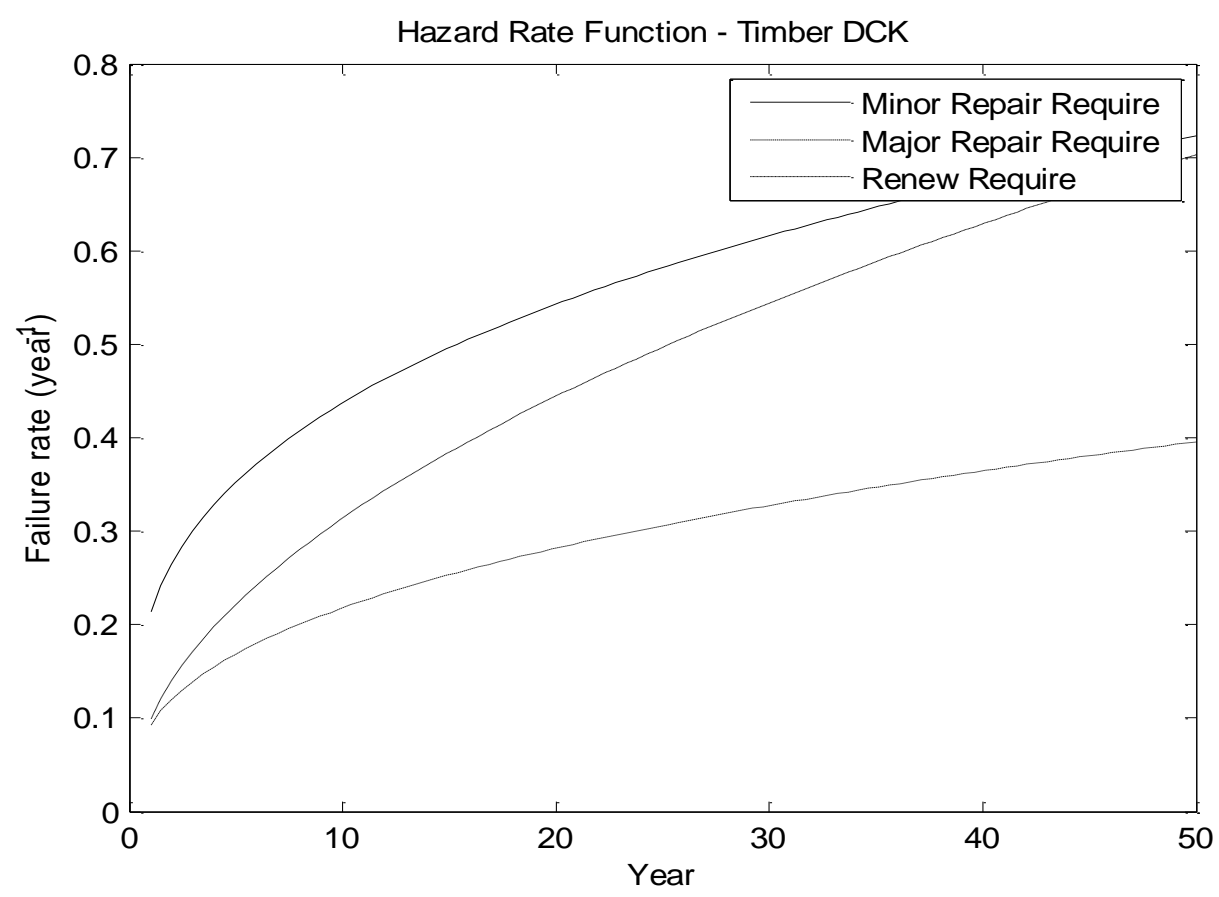

Figure A.29: Hazard rate function which shows the rates that an intervention is needed at different life-time

Timber deck result demonstrated a very short live comparing with deckings of other materials. Also the failure rates for reaching different conditions increase significantly with times. The results show that the characteristic time for a timber deck to be replaced is actually faster than the time for it to undergo major repairs. This suggests that the deck would actually need to be replaced before it needs major repairs. The reason for this is because timber materials have much shorter life span than metal and concrete, also timber is much harder to repair, that is once the material reaches a point of severe defects, the timber deck is usually replaced. This preferable option of repairs is demonstrated in Figure A.25. The number of replacements recorded in the database (more than 100 timber deck replacements) is much greater than the number of times major repair were carried out (20 timber deck major repairs). 
vi. Bearing

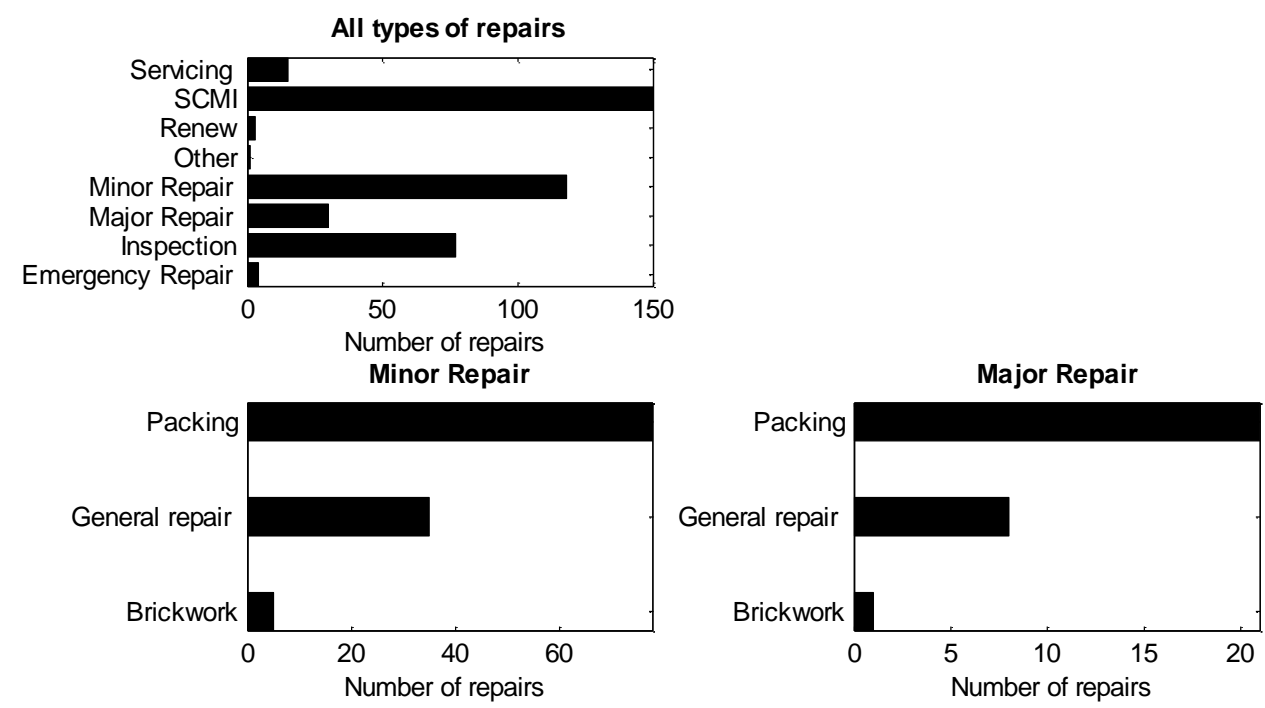

Figure A.30: Distributions of specific works for Metal Bearing

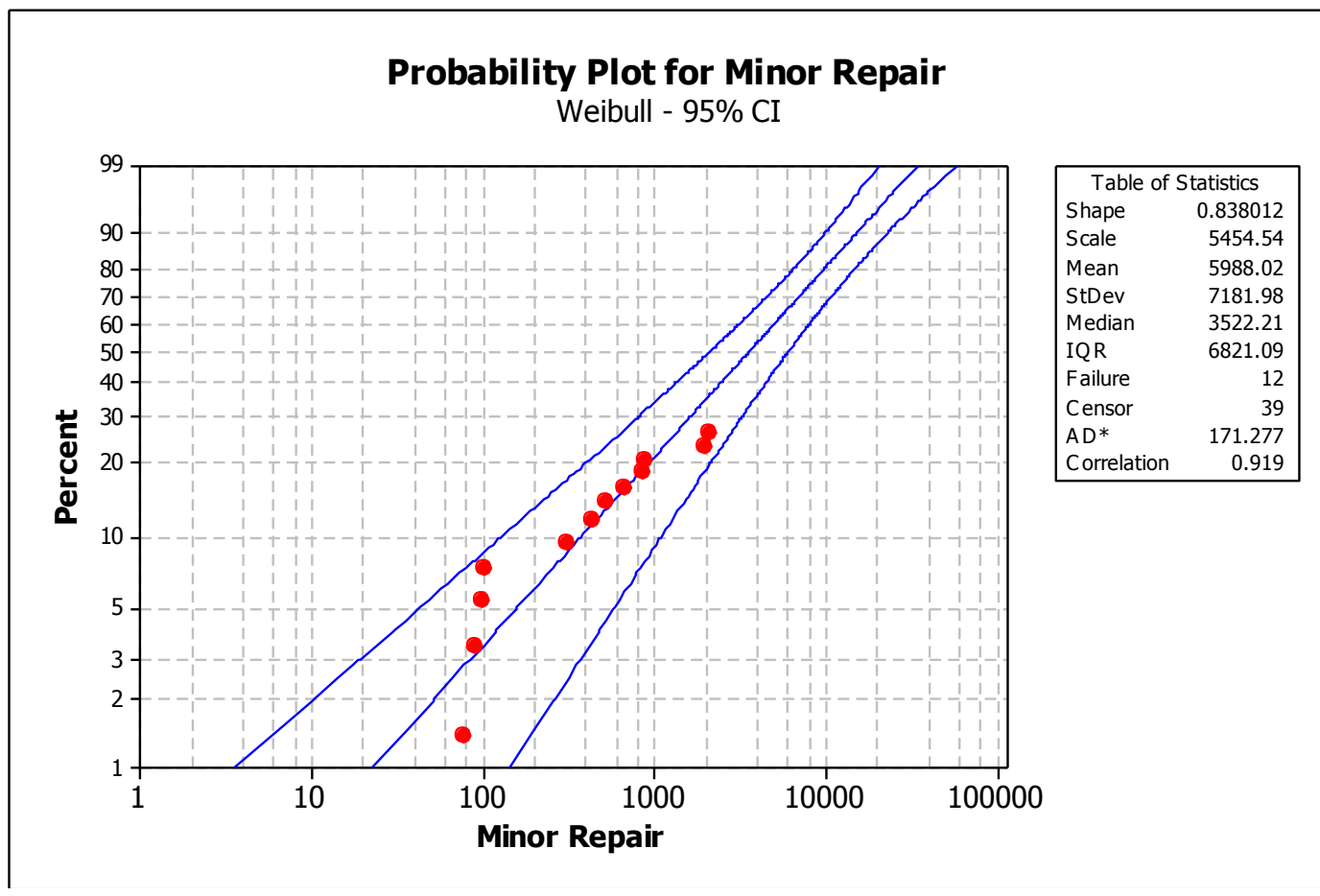

Figure A.31: Probability plot of the time to the point when Minor repair is needed 


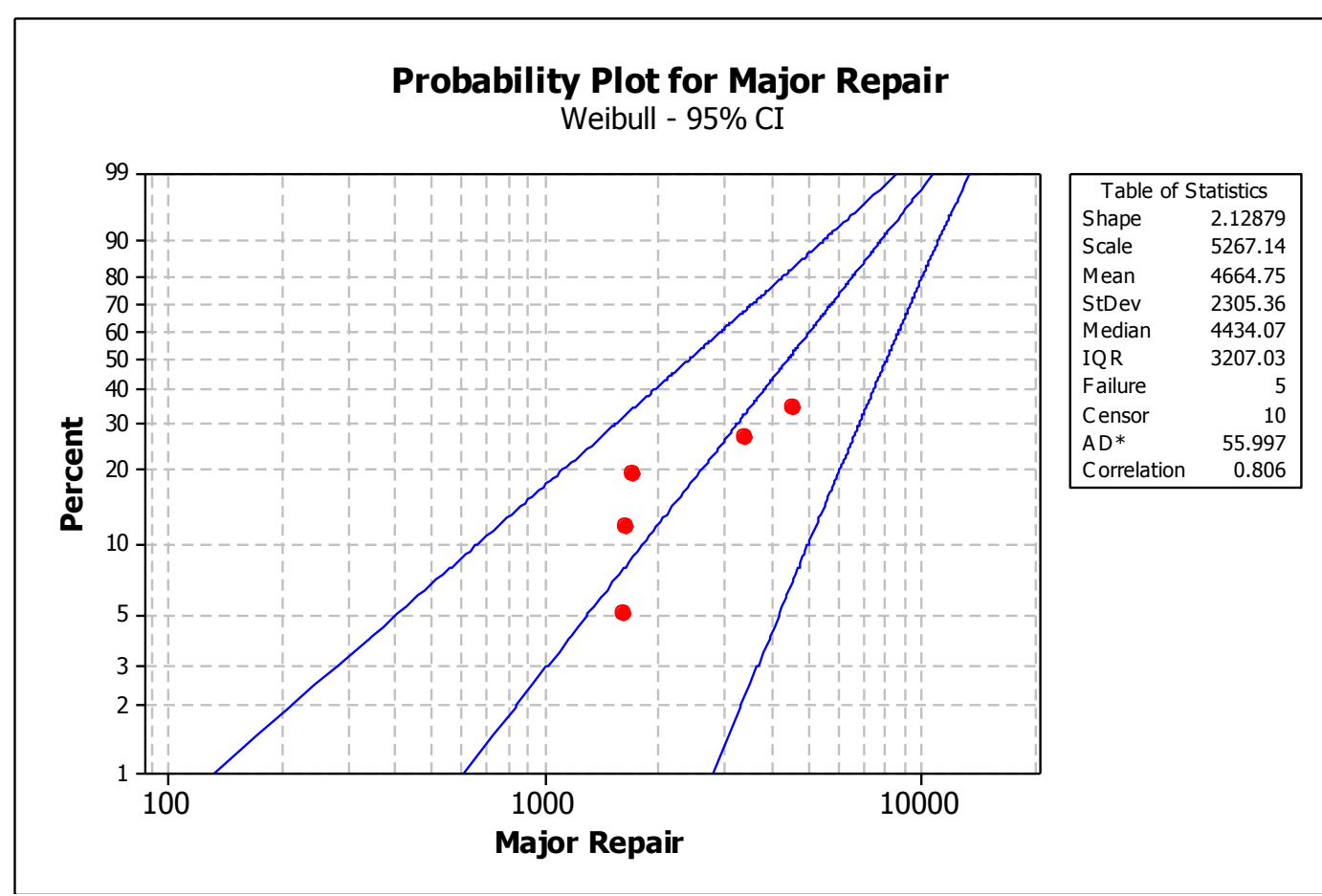

Figure A.32: Probability plot of the time to the point when Major repair is needed

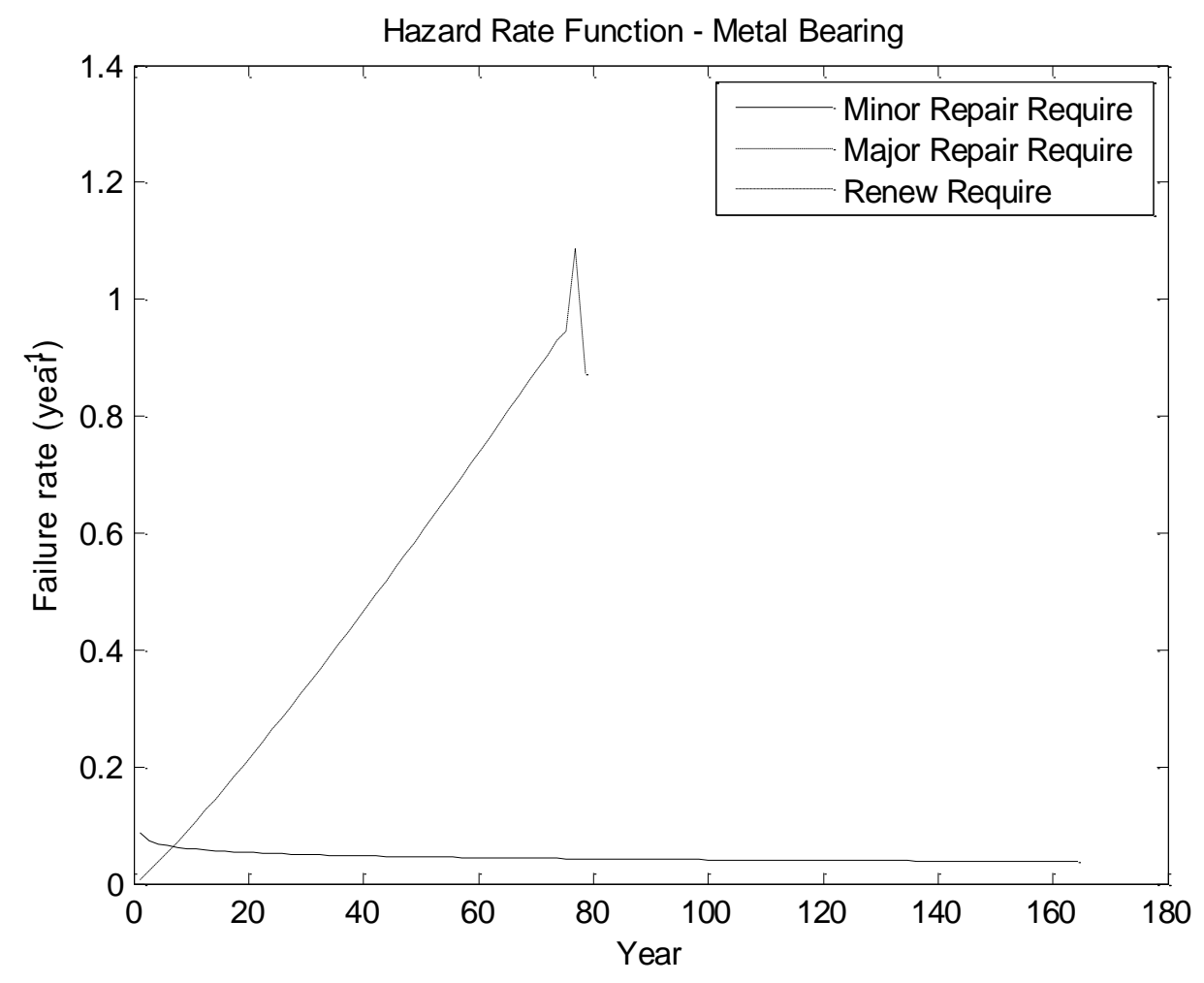

Figure A.33: Hazard rate function which shows the rates that an intervention is needed at different life-time

The rate at which the bearing would require a minor repair is almost constant at about 0.1 every year. Unexpectedly, it can be seen that the characteristic life of the bearing reaching a poor 
condition is actually shorter than that of reaching a good condition. The data that indicates a bearing major repair is often extracted from an entry that carries information about other repair works. Even though this entry is categorised in the database as major work, it might be that other works were major and the bearing repair might be opportunistic work. About $70 \%$ of bearing major repair data were extracted this way and since it is not possible to validate these entries, it is accepted that the data has influence these unexpected results.

\section{vii. Abutment}
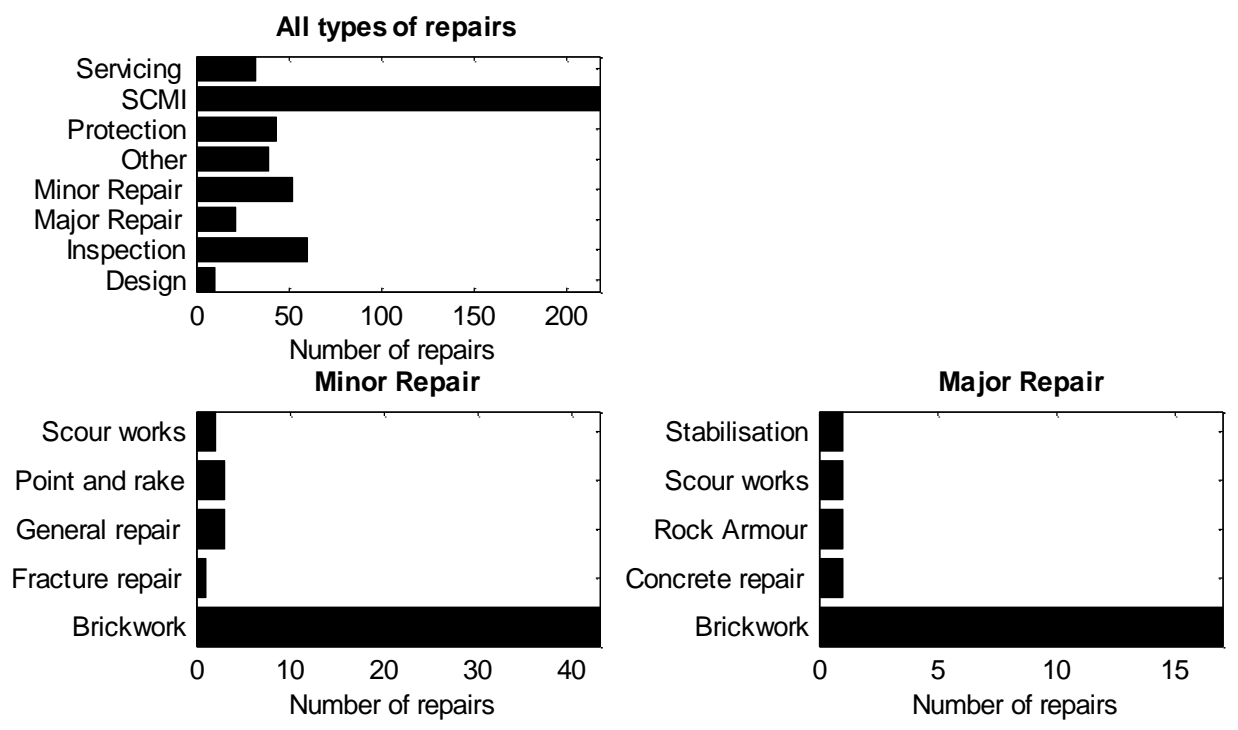

Figure A.34: Distributions of specific works for Masonry Abutment

The results obtained indicate that abutment requires much less maintenance than other bridge elements with the mean time of an abutment to require minor repair is about 50 years. There were no data to allow the rate of abutment replacement to be calculated, which again agrees with the fact that abutment almost never require complete replacement, unless it is a complete demolition of the entire bridge due to upgrade or natural disaster. 


\section{Appendix B Environment adjustment factor}

The environment adjustment factors are provided by the experts' opinions (Network Rail, 2010a), which reflect the differences in degradation rates of the bridge components operating under different environments.

\begin{tabular}{|c|c|c|c|}
\hline \multirow{2}{*}{$\begin{array}{c}\text { Element } \\
\text { Material }\end{array}$} & \multicolumn{3}{|c|}{ Environment Adjustment Factor (A $\left.\mathbf{A}_{\mathrm{E}}\right)$} \\
\cline { 2 - 4 } Metal & Aggressive & Moderate & Benign \\
\hline Concrete & 1 & 0.678571 & 0.357143 \\
\hline Masonry & 1 & 0.683099 & 0.366197 \\
\hline Timber & 1 & 0.684564 & 0.369128 \\
\hline
\end{tabular}




\section{Appendix C Markov bridge model}

\section{C-1 Transition rate matrix}

The transition rate matrix for a bridge system contains two major elements is a $32 \times 32$ matrix since there are 32 Markov states in the model. The matrix is given below, where:

\footnotetext{
$\lambda_{1}^{1}$ is the transition rate from the 'as new' state to the good state for component 1 , $\lambda_{2}^{1}$ is the transition rate from the good state to the poor state for component 1 , $\lambda_{3}^{1}$ is the transition rate from the poor state to the very poor state for component 1 , $\lambda_{1}^{2}$ is the transition rate from the 'as new' state to the good state for component 2, $\lambda_{2}^{2}$ is the transition rate from the good state to the poor state for component 2 , $\lambda_{3}^{2}$ is the transition rate from the poor state to the very poor state for component 2 ,

$v_{1}^{1}$ is the transition rate from the 'as new' state to the good state for component 1 , $v_{2}^{1}$ is the transition rate from the good state to the poor state for component 1 , $v_{3}^{1}$ is the transition rate from the poor state to the very poor state for component 1 , $v_{1}^{2}$ is the transition rate from the 'as new' state to the good state for component 2, $v_{2}^{2}$ is the transition rate from the good state to the poor state for component 2 , $v_{3}^{2}$ is the transition rate from the poor state to the very poor state for component 2 ,
} 


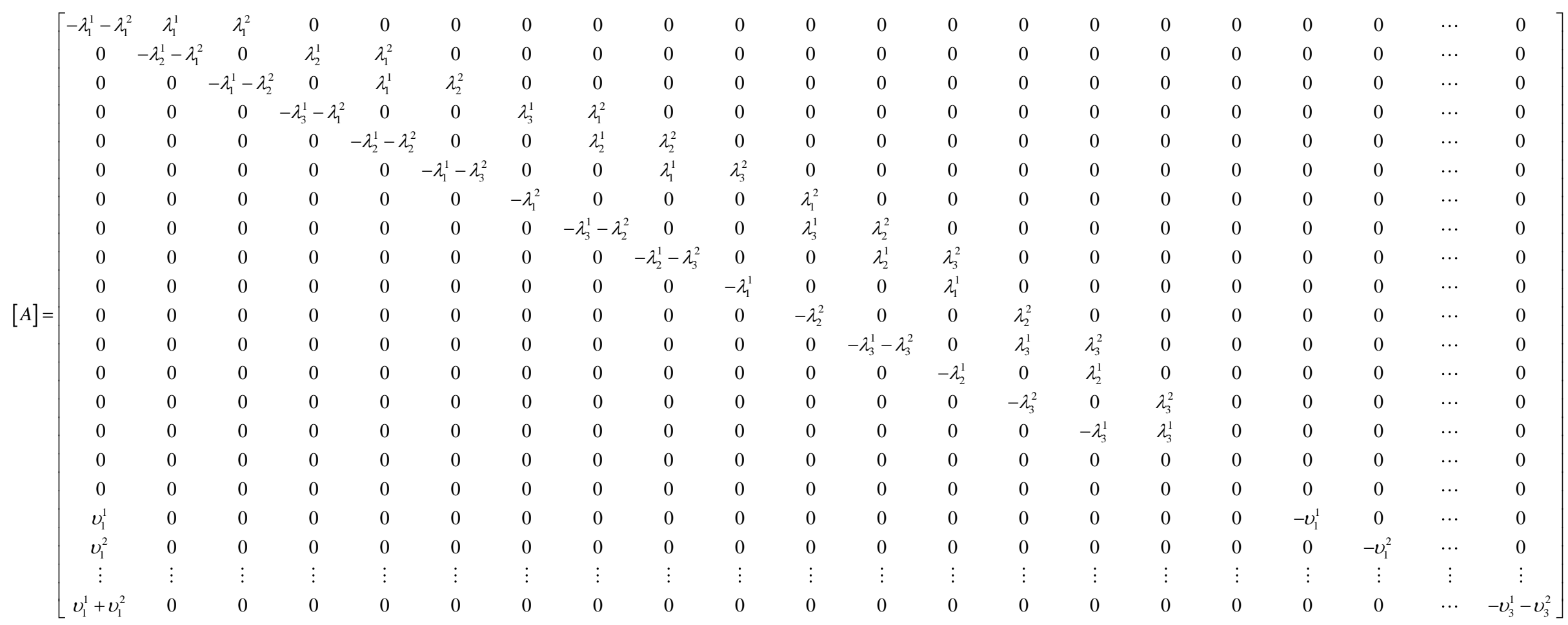




\section{C-2 Expected WLCCs}

Expected WLLCs for all modelled bridge components under different maintenance strategies.

\begin{tabular}{|c|c|c|c|c|c|}
\hline Component & Strategy 1 & Strategy 2 & $\begin{array}{c}\text { Strategy 2 } \\
\text { with } \\
\text { opportunistic } \\
\text { maintenance }\end{array}$ & Strategy 3 & $\begin{array}{c}\text { Strategy 3 } \\
\text { with } \\
\text { opportunistic } \\
\text { maintenance }\end{array}$ \\
\hline DCK & 20786 & 23660 & 24474 & 31635 & 31819 \\
\hline MGE1 & 25095 & 28807 & 27529 & 8767 & 26036 \\
\hline MGI1 & 39978 & 40521 & 42412 & 15396 & 39442 \\
\hline MGE2 & 25095 & 28807 & 27529 & 8767 & 26036 \\
\hline BGL1 & 51763 & 63611 & 64917 & 51016 & 60085 \\
\hline BGL2 & 51763 & 63611 & 64917 & 51016 & 60085 \\
\hline ABT1 & 5669 & 3256 & 5727 & 496 & 5277 \\
\hline ABT2 & $\underline{5669}$ & $\underline{3256}$ & $\underline{5727}$ & $\underline{496}$ & $\underline{5277}$ \\
\hline Total & 235366 & 265079 & 272782 & 177141 & 263605 \\
\hline
\end{tabular}

Table C.1: WLCC (£) for all bridge components under different maintenance strategies
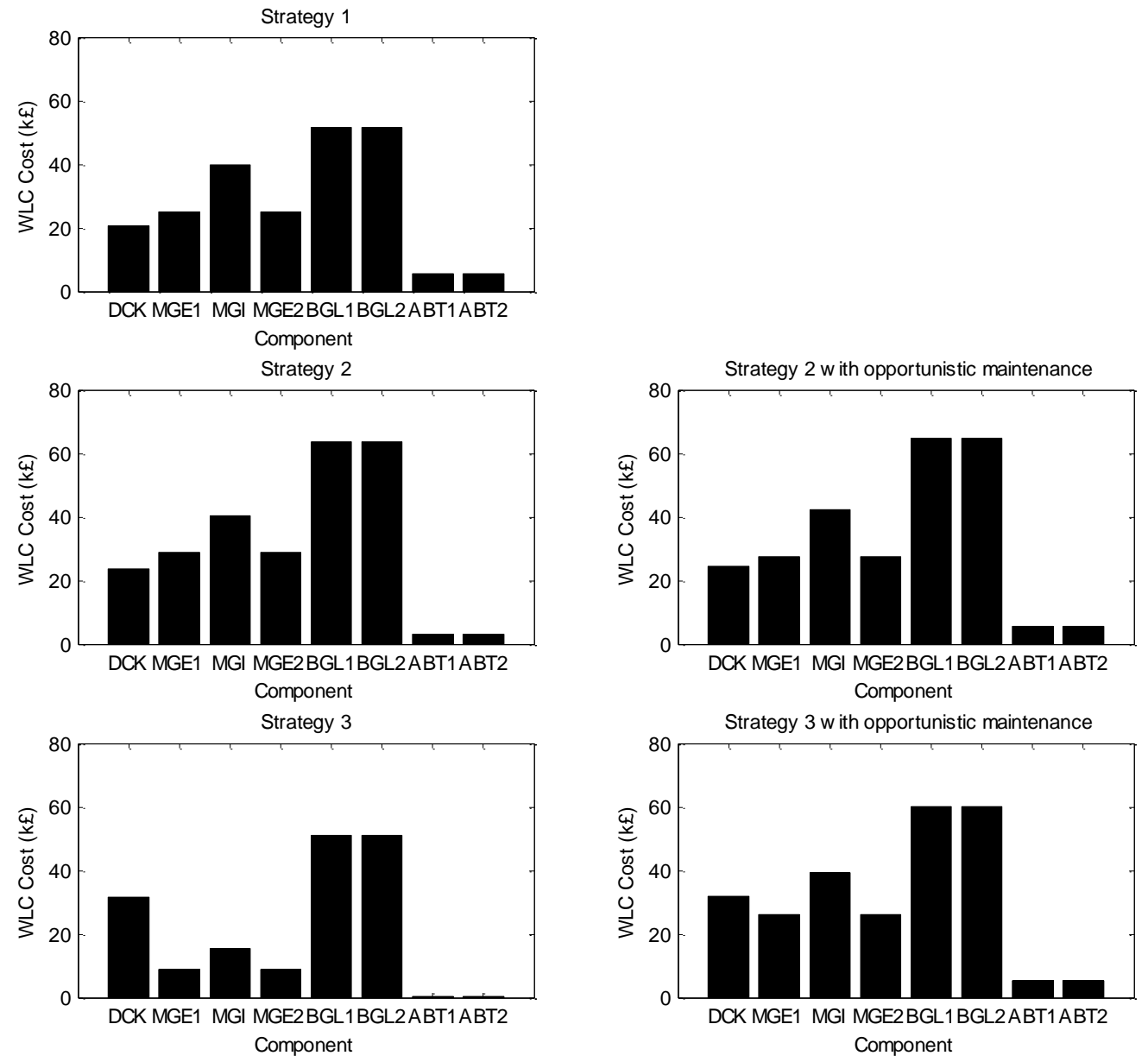

Figure C.1: Expected WLCC (£) for each element with different maintenance strategies 


\section{Appendix D Petri-Net bridge model}

\section{D-1 Degradation of protective coating and its effects on metal element}

The degradation rates of the coating were estimated by studying the standard document (RT/CE/C/O02) and consulting a group of bridge engineering experts. A suggested service life of a system of coating is about 25 years (Table 3 in RT/CE/C/002). Given that there are five states in the condition of the coating (Network Rail, 2004b), it was assumed that the mean time of the coating staying in each condition state is around 5 years. It is also assumed that the degradation rate of the coating is constant.

The cost of applying the coating was estimated from the database as well as given in the standard (RT/CE/S/039). The estimated cost is around $£ 20 / \mathrm{m}^{2}$ (figure estimated in 2001).

There is not enough data to support the determination of the coating degradation. The degradation rates were therefore estimated by consulting with a group of bridge engineer experts which resulted in the data shown in Table D.1. As the coating condition degrades to poorer conditions, it was assumed that the deterioration rates of the metal element will increase by $5 \%$. This effect is reflected by reducing the scale parameters of the Weibull distributions which govern the transition times between each element's condition states.

\begin{tabular}{|l|c|c|c|}
\hline \multirow{2}{*}{ Coating condition } & \multicolumn{2}{|c|}{$\begin{array}{c}\text { Coating degradation } \\
\text { rates }\end{array}$} & $\begin{array}{c}\text { Effects on the metal } \\
\text { degradation rates }\end{array}$ \\
\cline { 2 - 3 } & Beta & Eta (years) & \\
\hline New & - & - & As estimated \\
\hline Coating intact & 1.0 & 5 & $5 \%$ faster \\
\hline Flacking or blistering & 1.0 & 5 & $10 \%$ faster \\
\hline Loss of coating & 1.0 & 5 & $15 \%$ faster \\
\hline Complete loss of coating & 1.0 & 5 & $20 \%$ faster \\
\hline
\end{tabular}

Table D.1: Degradation rates of metal coating and the effects of coating condition on the degradation rates of metal element. 


\section{D-2 Model results - element analysis for all bridge components}

This section presents the simulation results for all 8 modelled bridge components in the PN bridge model. The simulation investigate the effects of maintenance strategy 1 , which is repair as soon as possible.

\section{Bridge Deck (DCK)}
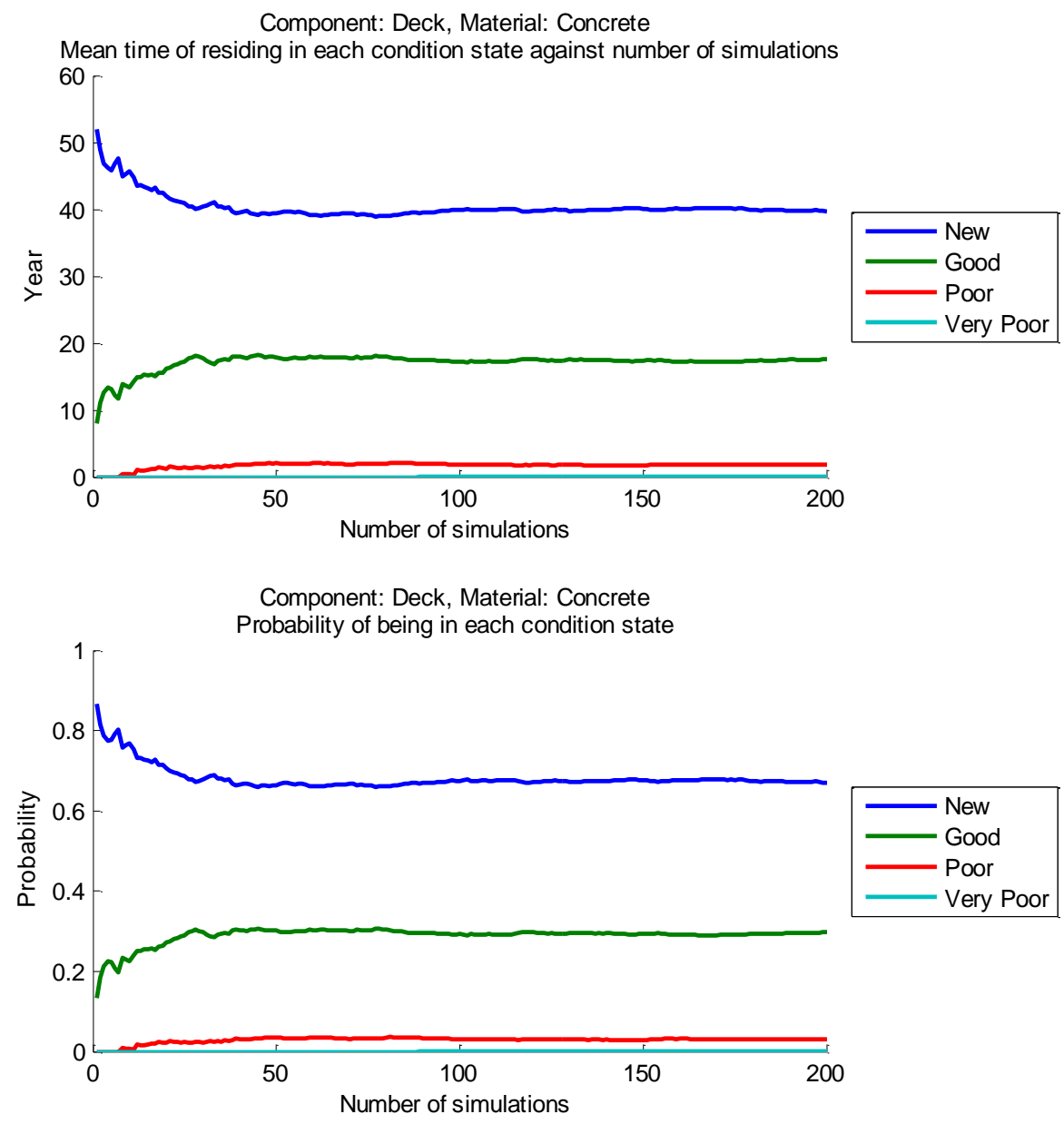

Component: Deck, Material: Concrete

Average number of interventions per lifetime against the number of simulatons 3

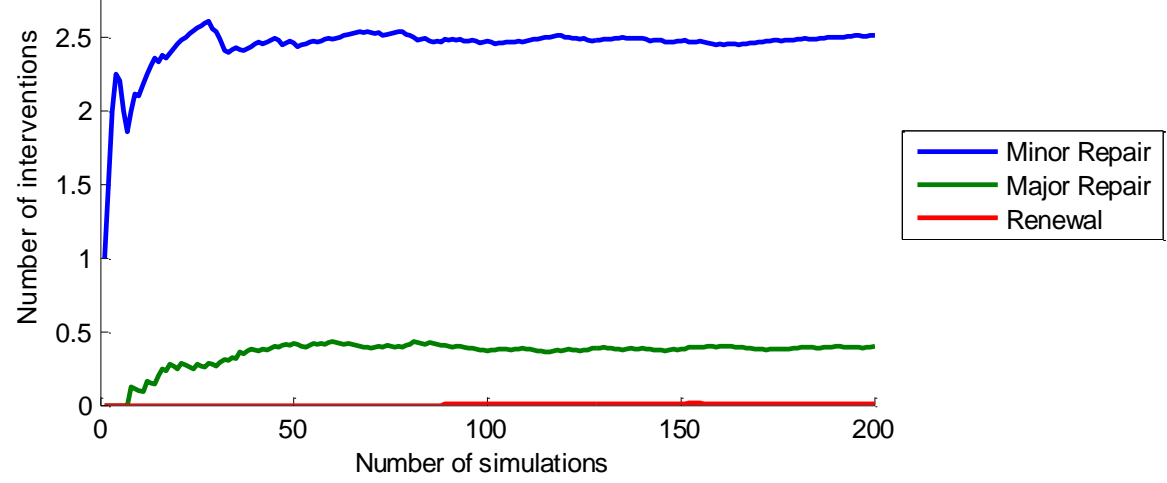




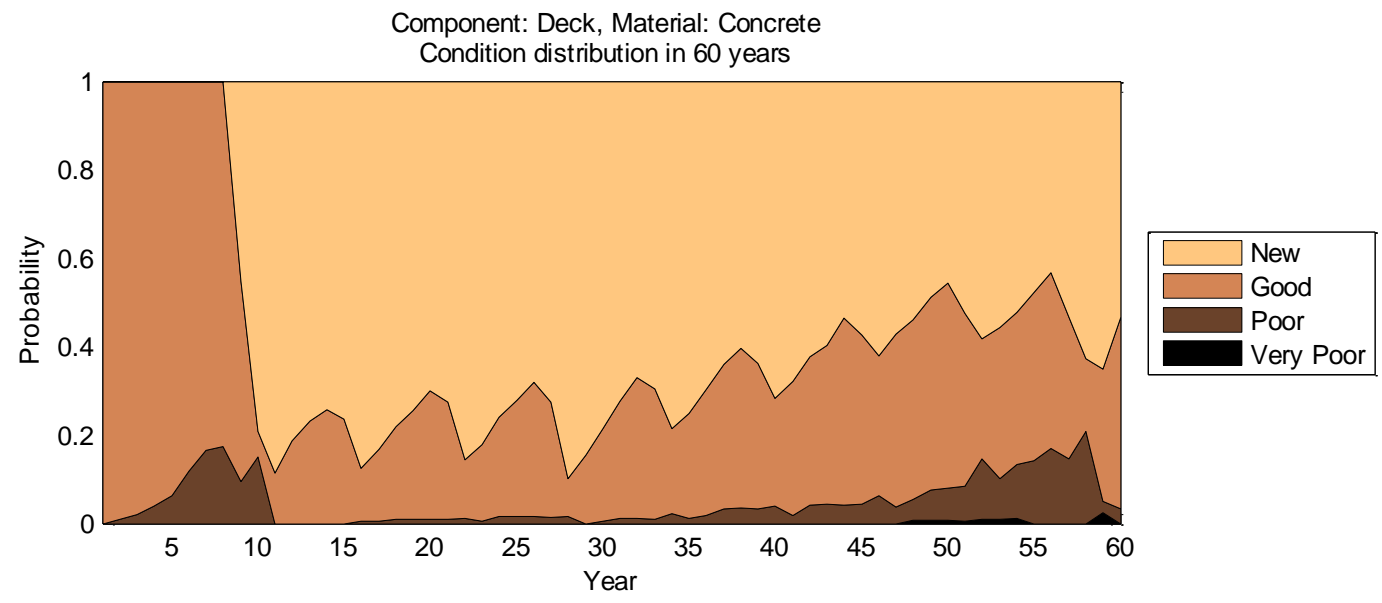




\section{External Main Girder 1 (MGE1)}

Component: Girder, Material: Metal

Mean time of residing in each condition state against number of simulations 50 .
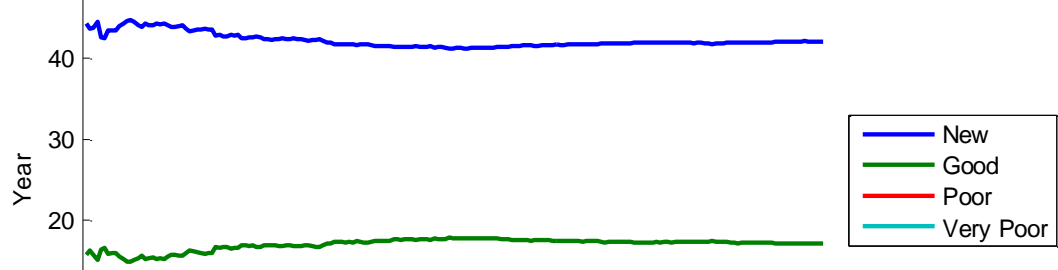

10 .

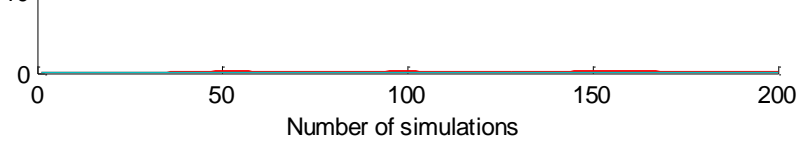

Component: Girder, Material: Metal Probability of being in each condition state
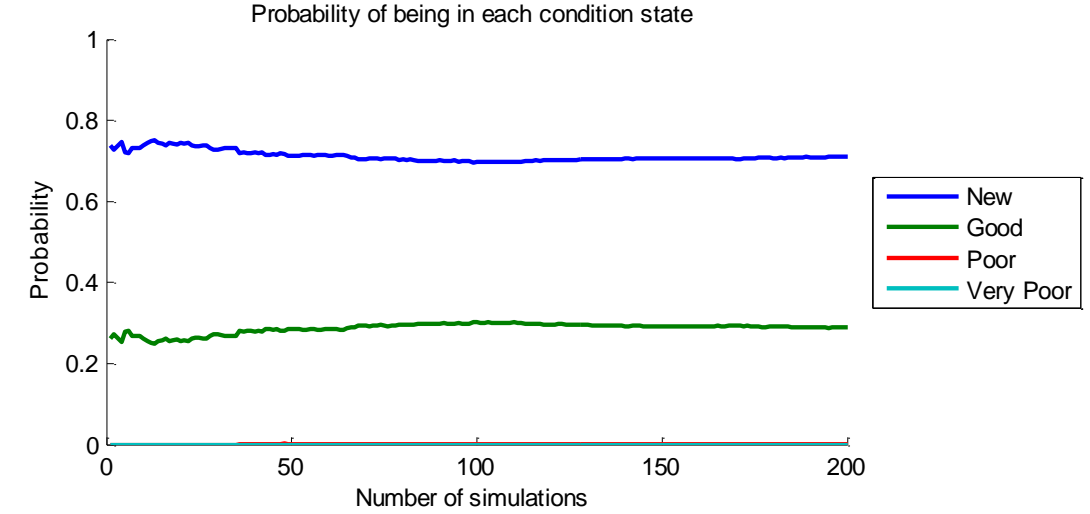

Component: Girder, Material: Metal

Average number of interventions per lifetime against the number of simulatons
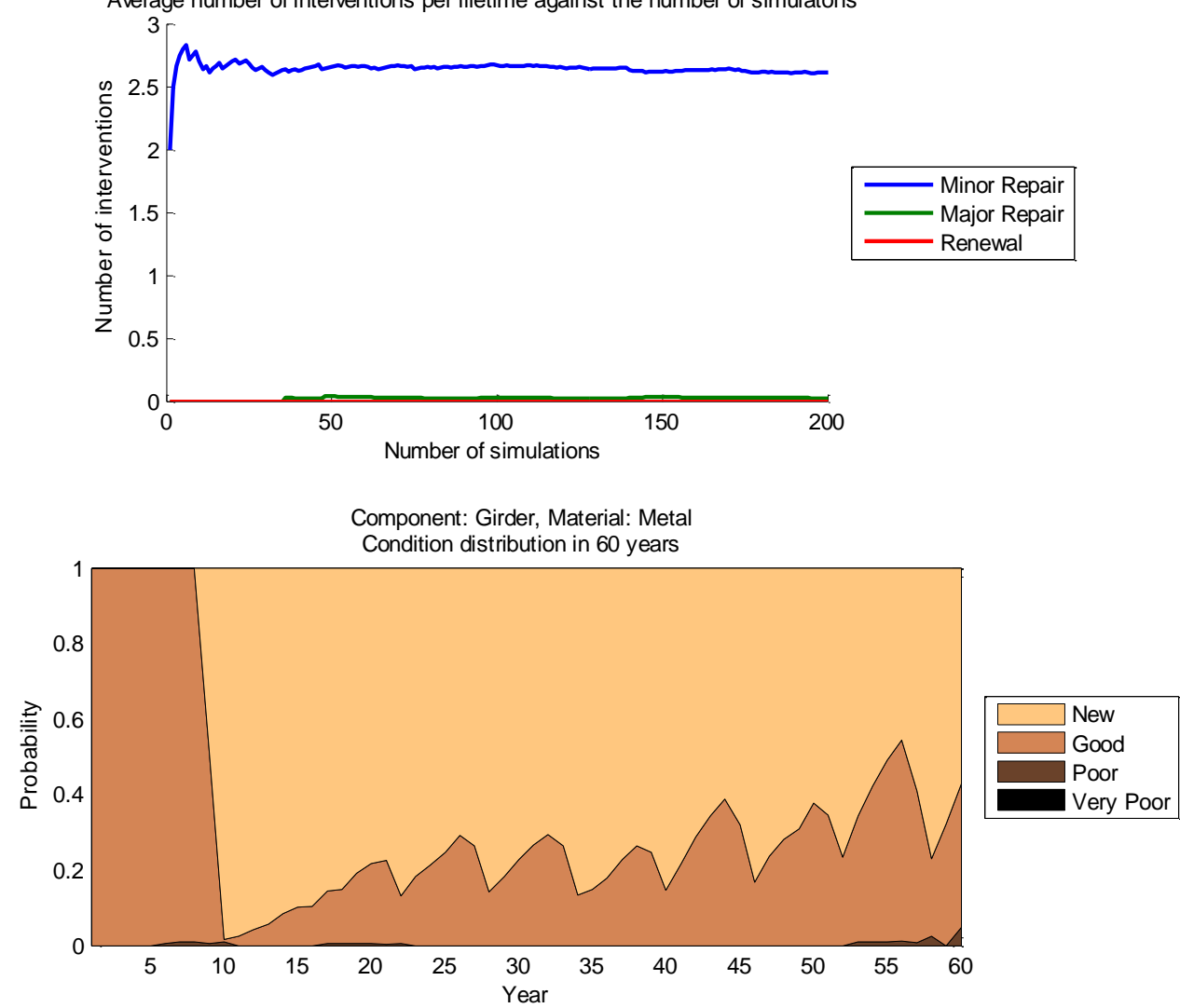
Internal Main Girder (MGI)

Component: Girder, Material: Metal

Mean time of residing in each condition state against number of simulations

50

40

$\stackrel{\frac{\pi}{\pi}}{\searrow}_{20}^{30}$.
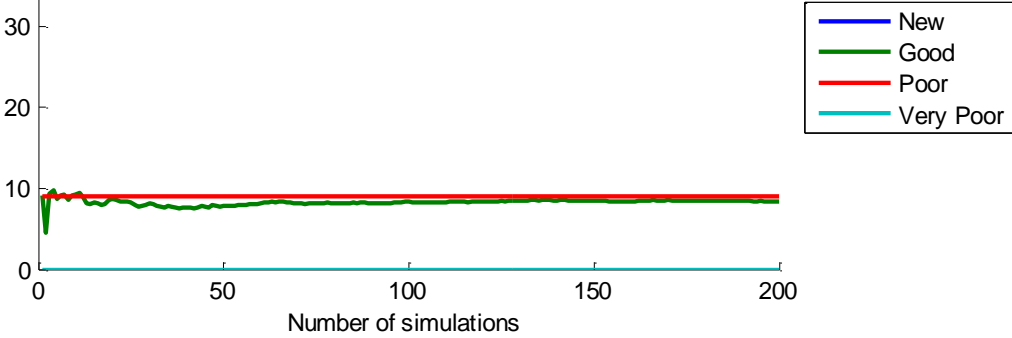

Component: Girder, Material: Metal

Probability of being in each condition state
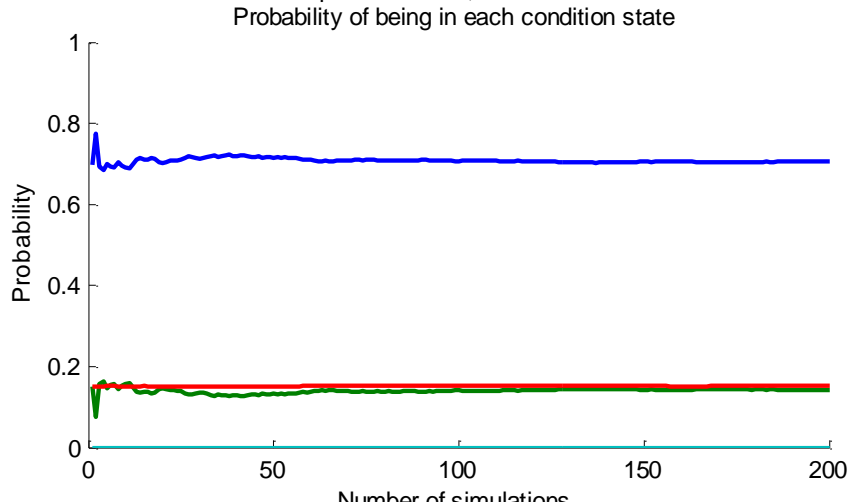

Component: Girder, Material: Metal

Average number of interventions per lifetime against the number of simulatons
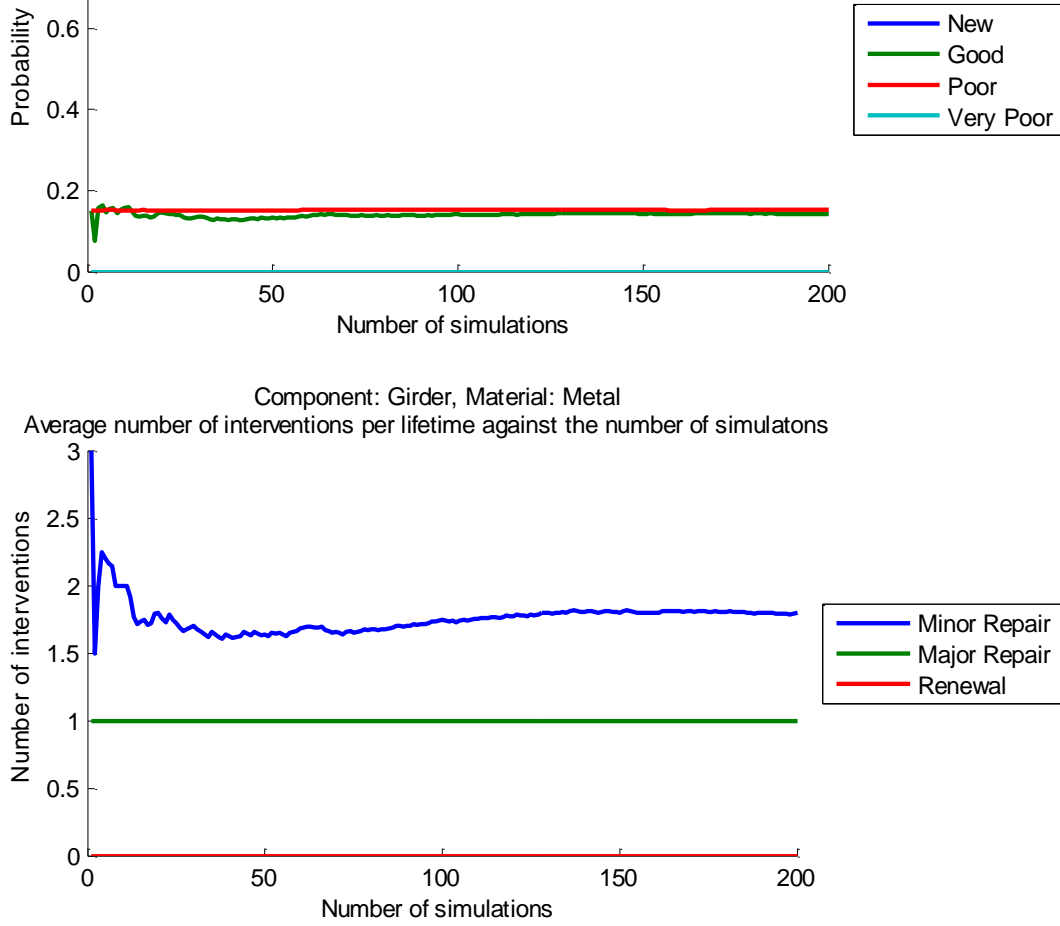

Very Poor

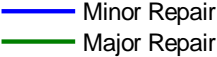

Renewal

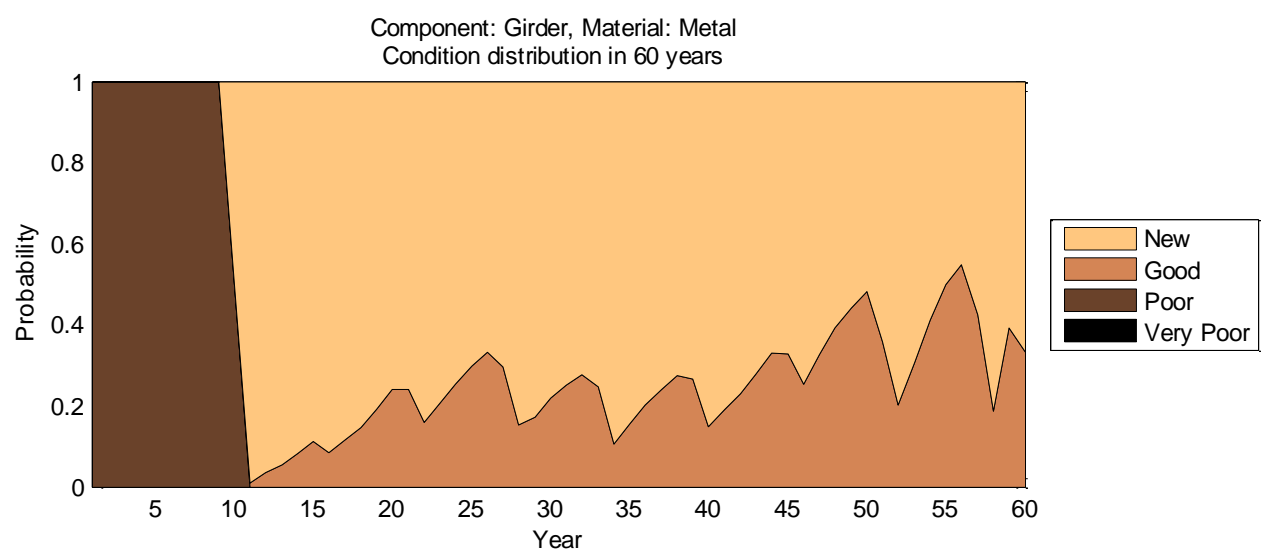




\section{External Main Girder (MGE2)}

Component: Girder, Material: Metal

Mean time of residing in each condition state against number of simulations

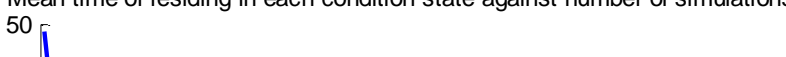

40
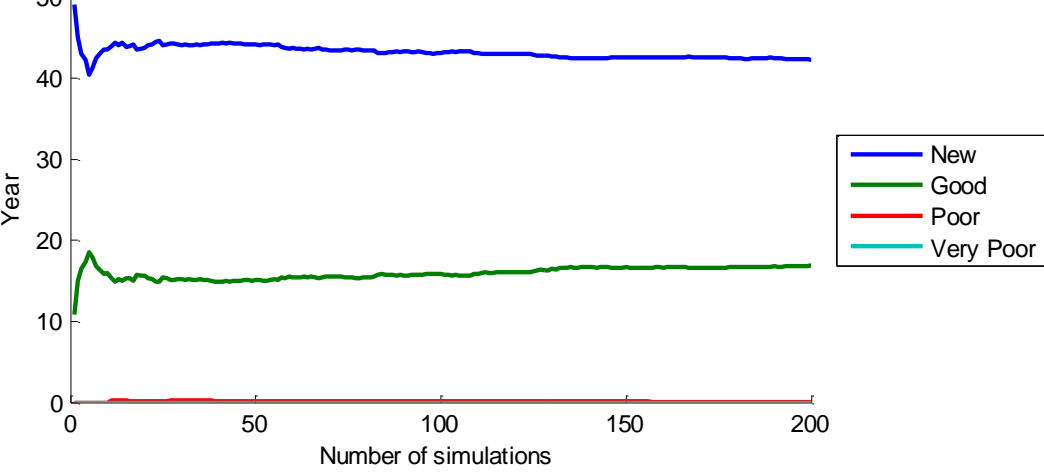

Component: Girder, Material: Metal

Probability of being in each condition state

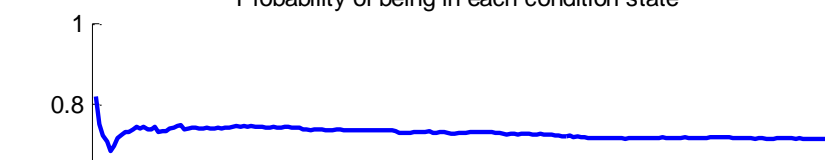

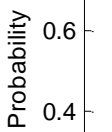

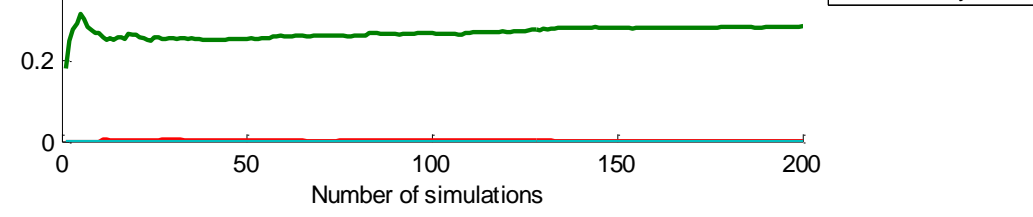

5

Component: Girder, Material: Metal

Average number of interventions per lifetime against the number of simulatons 3 .

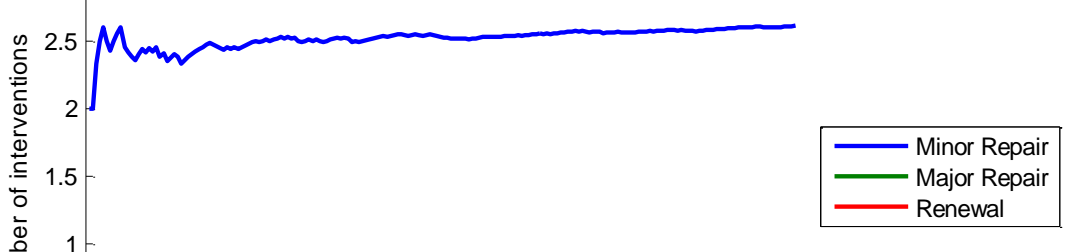

$\sum^{\frac{5}{z}}$

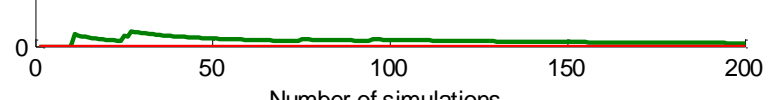

Component: Girder, Material: Metal Condition distribution in 60 years

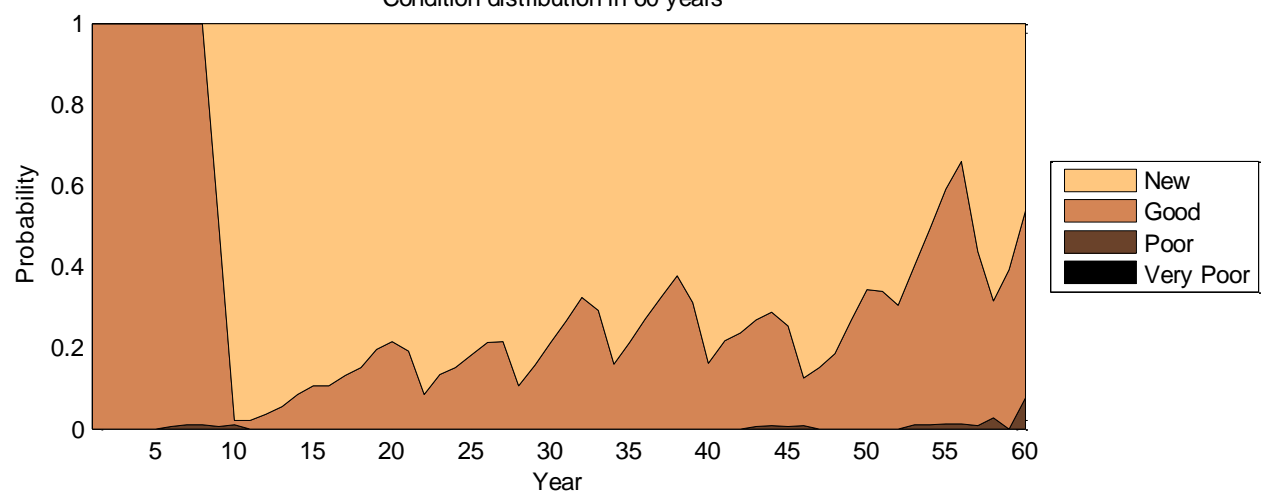




\section{Bearing 1 (BGL1)}

Component: Bearing, Material: Metal

Mean time of residing in each condition state against number of simulations 40
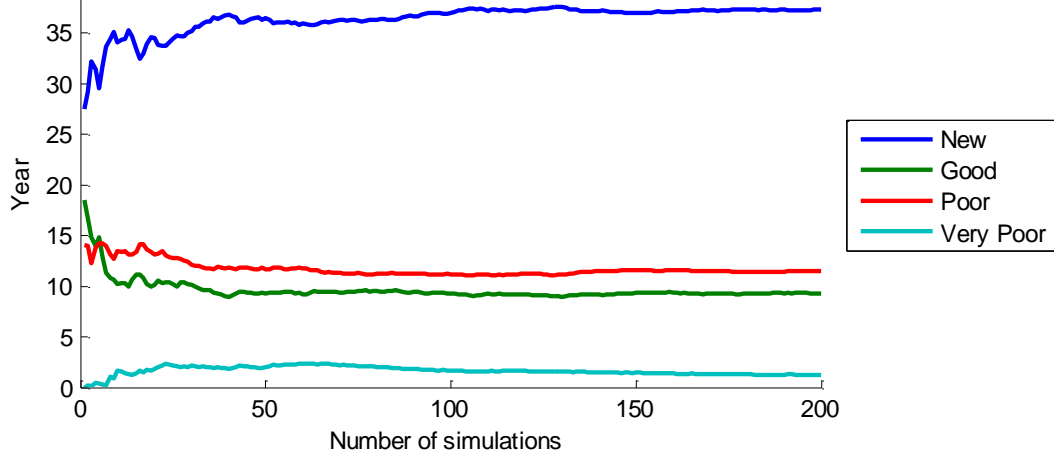

Component: Bearing, Material: Metal Probability of being in each condition state
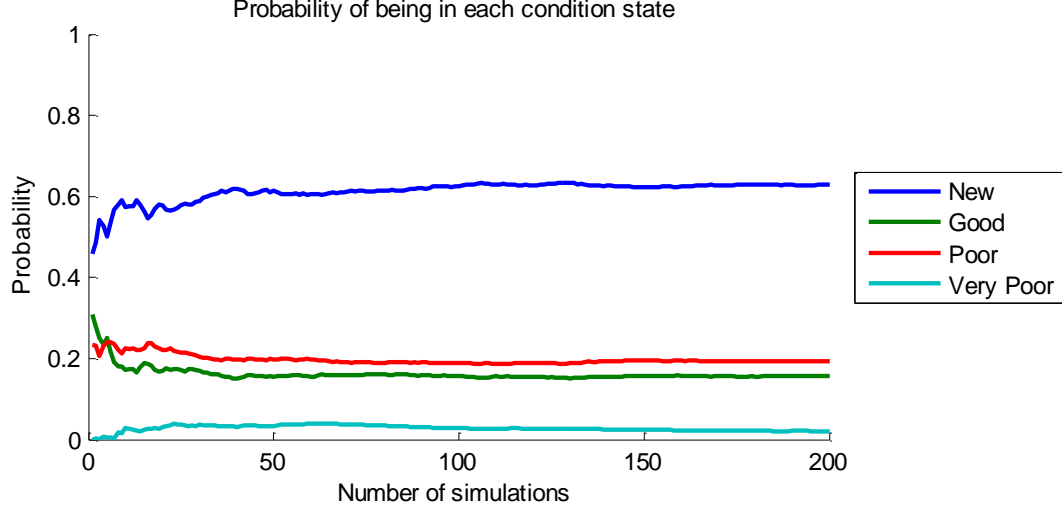

Component: Bearing, Material: Meta

Average number of interventions per lifetime against the number of simulatons

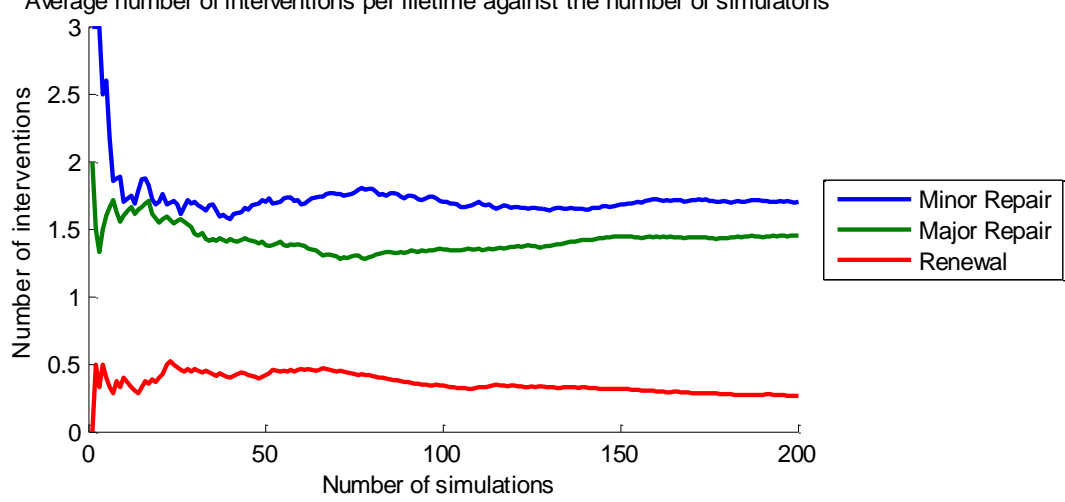

Component: Bearing, Material: Metal Condition distribution in 60 years

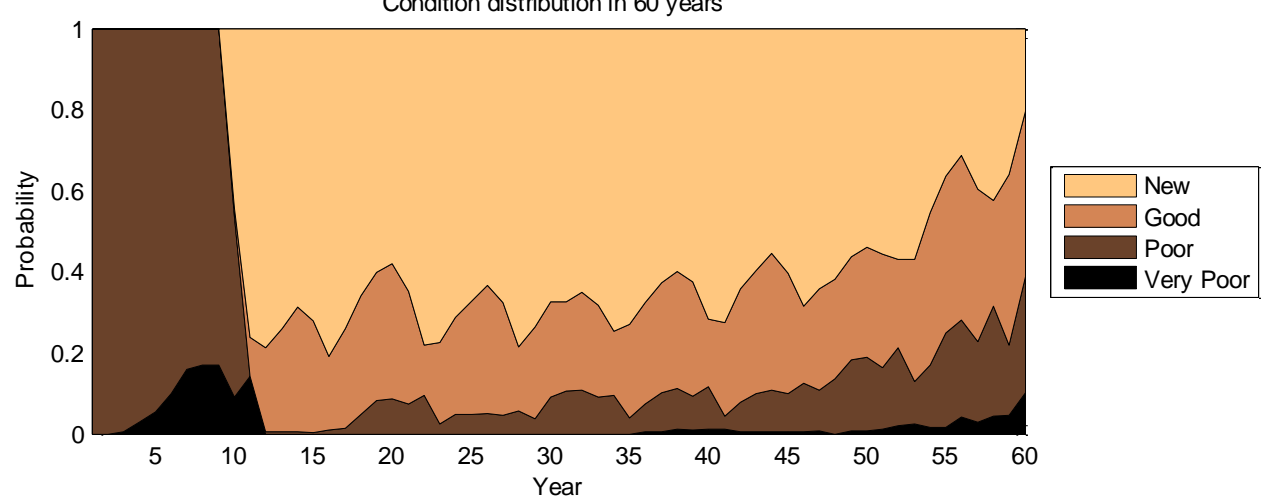




\section{Bearing 2 (BGL2)}

Component: Bearing, Material: Metal

Mean time of residing in each condition state against number of simulations 50

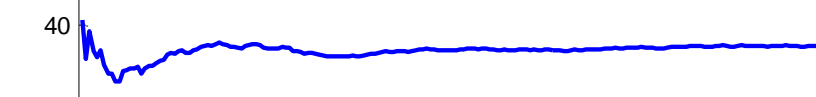

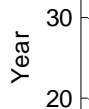
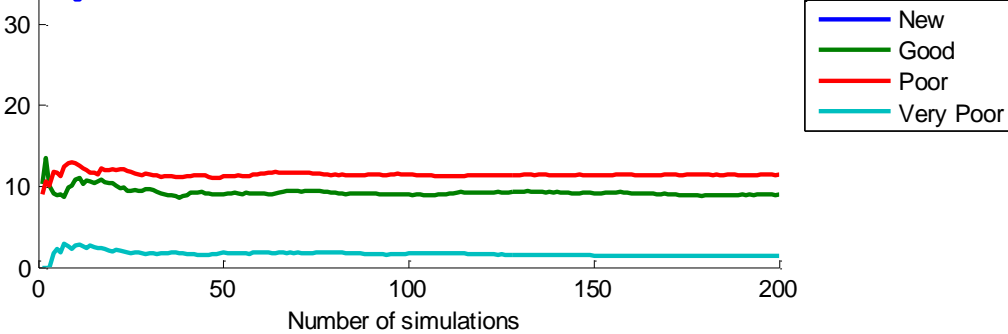

Component: Bearing, Material: Metal

Probability of being in each condition state

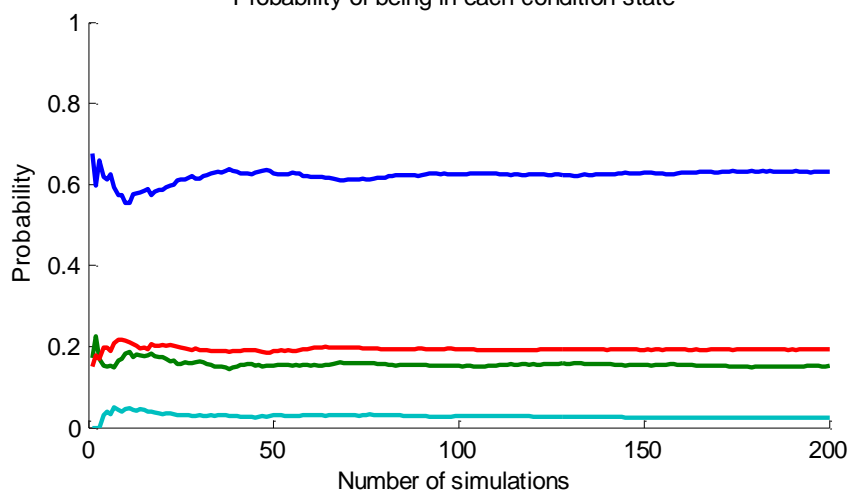

Component: Bearing, Material: Meta

Average number of interventions per lifetime against the number of simulatons
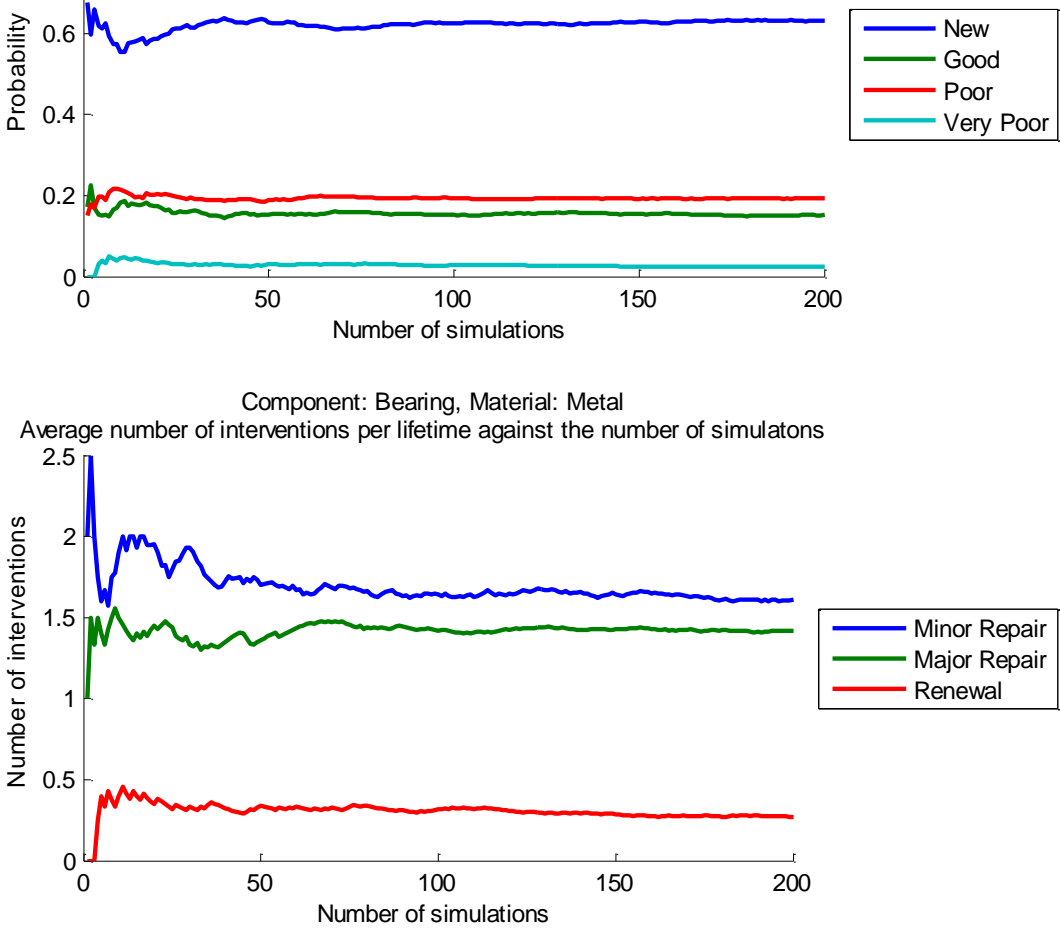

Very Poor

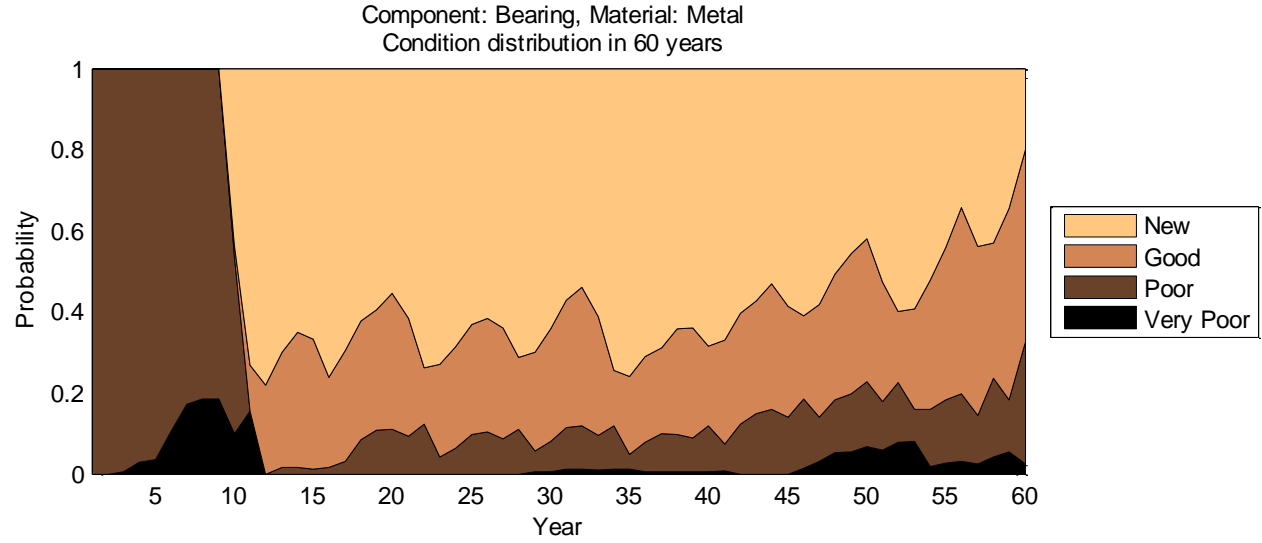


Abutment 1 (ABT1)

Component: Abutment, Material: Masonry

Mean time of residing in each condition state against number of simulations 60 .
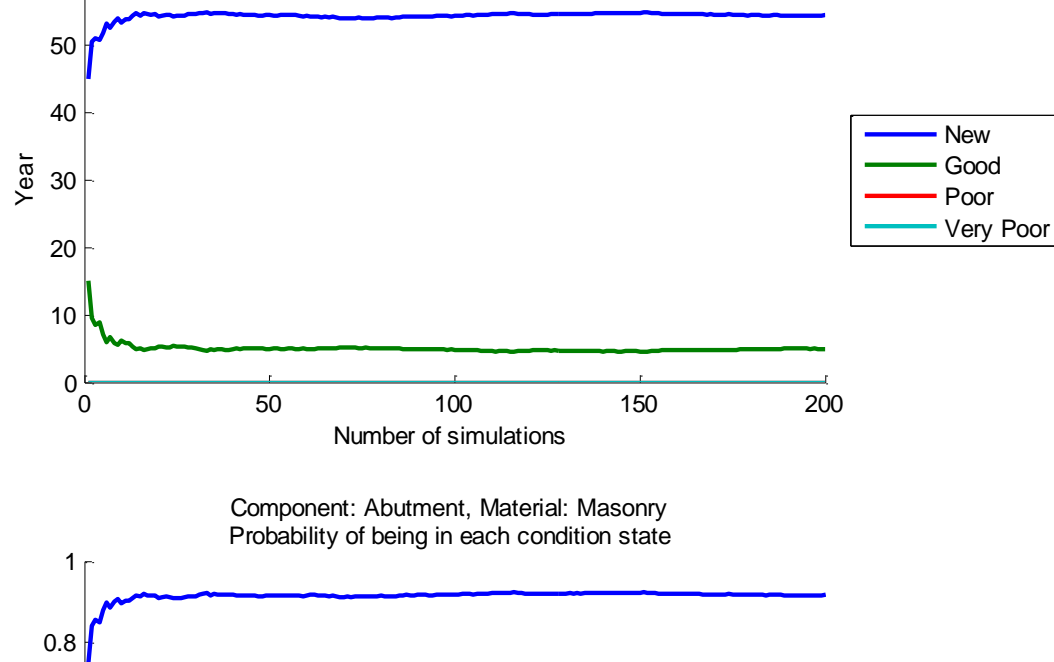

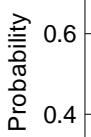
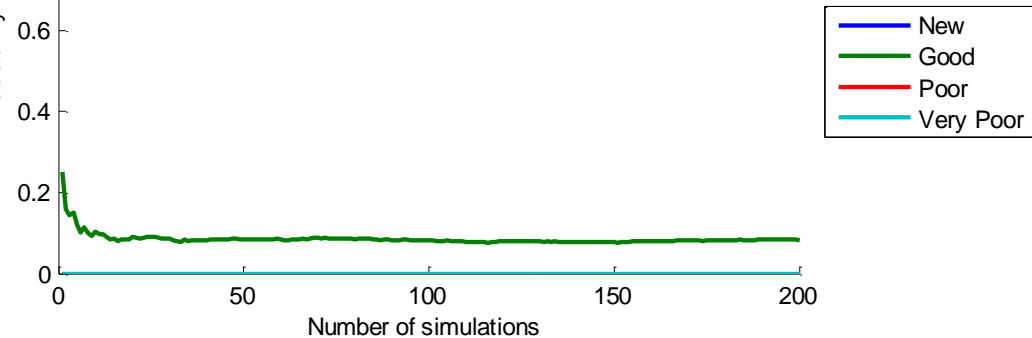

Component: Abutment, Material: Masonry

Average number of interventions per lifetime against the number of simulatons
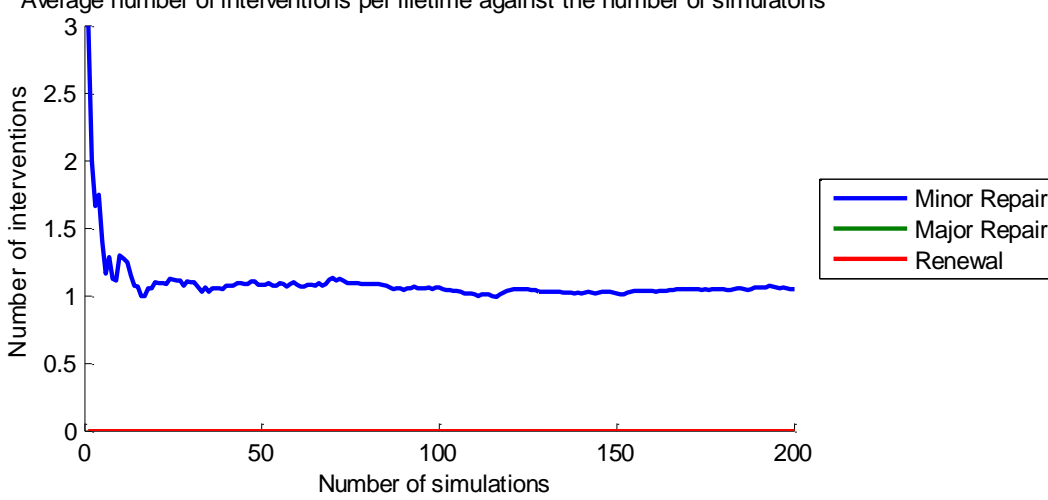

Component: Abutment, Material: Masonry Condition distribution in 60 years

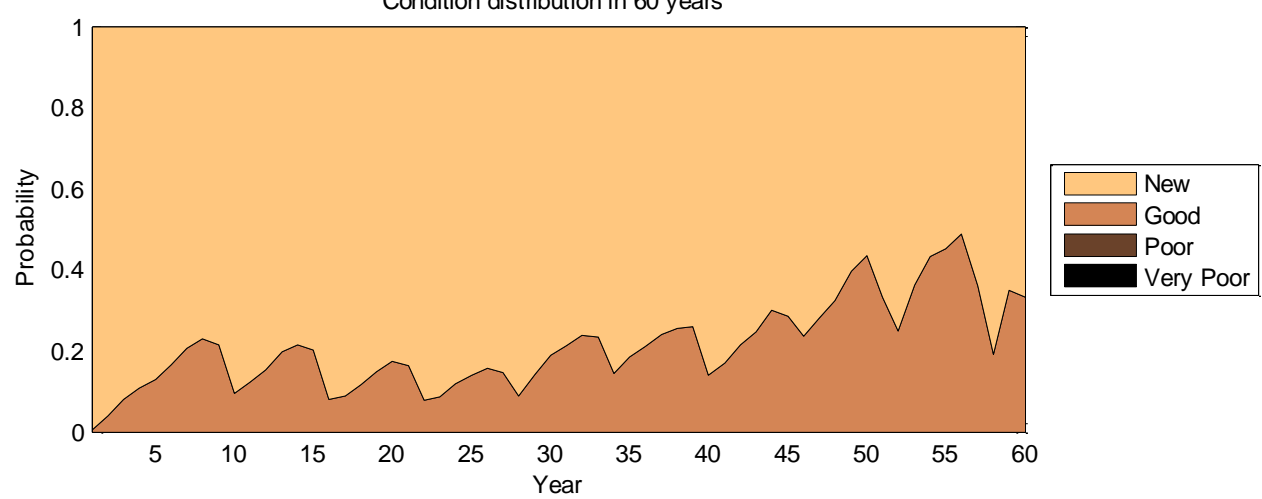


Abutment 2 (ABT2)

Component: Abutment, Material: Masonry

Mean time of residing in each condition state against number of simulations
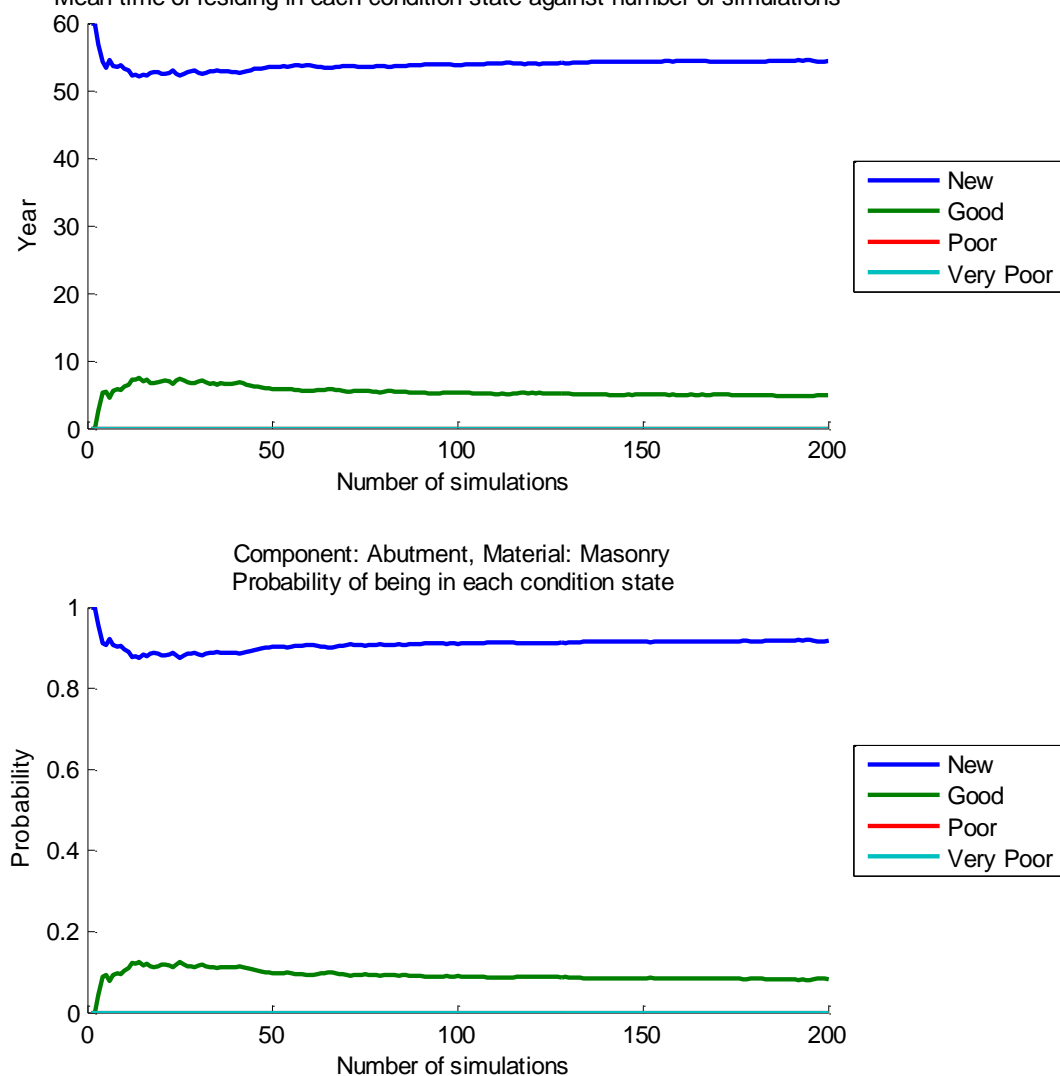

Component: Abutment, Material: Masonry

Average number of interventions per lifetime against the number of simulatons

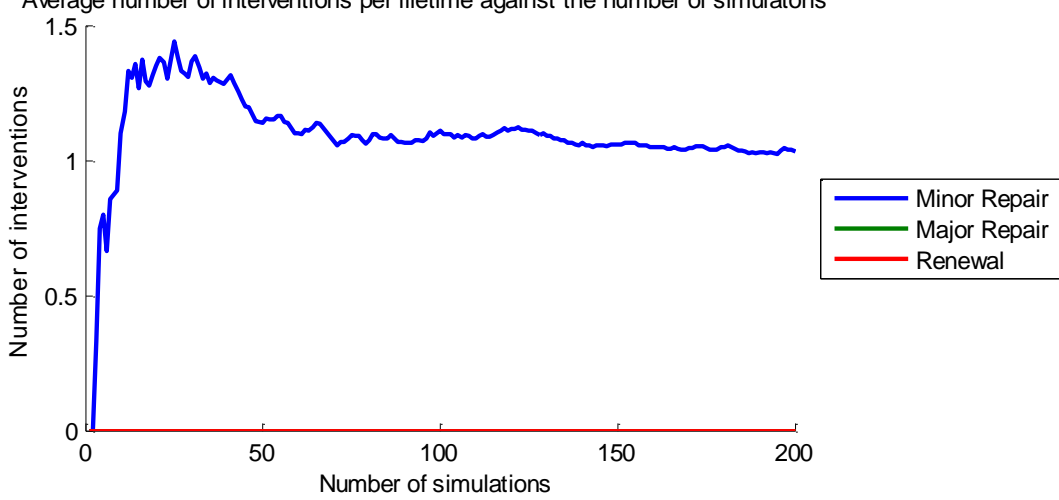

Component: Abutment, Material: Masonry Condition distribution in 60 years

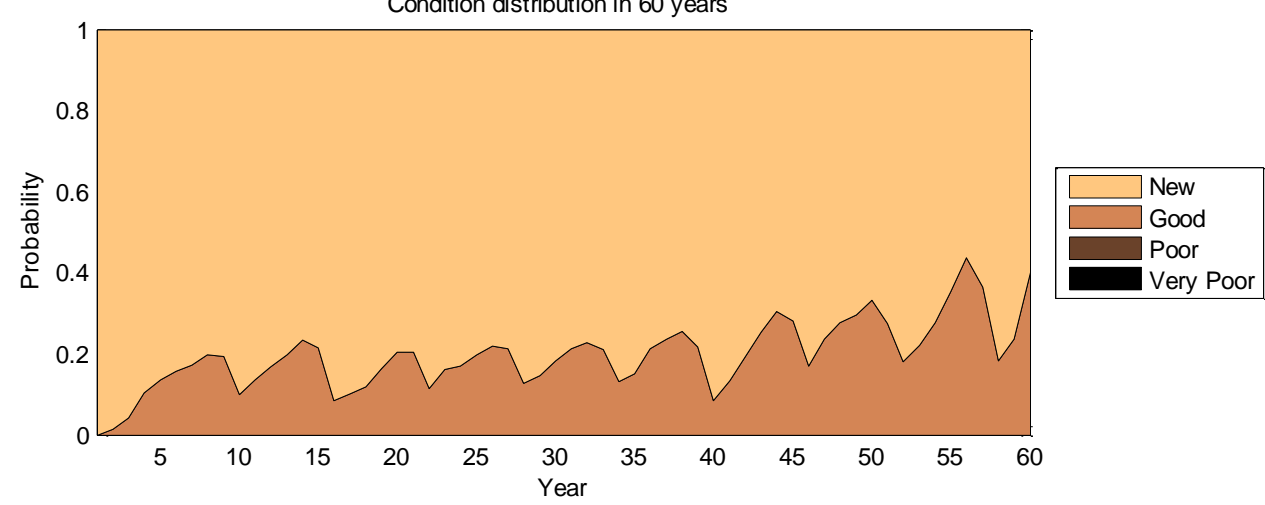




\section{D-3 Model results - system analysis for varying maintenance policies}

\section{Intervention option 1}

\begin{tabular}{|c|c|c|c|c|c|c|c|c|c|}
\hline & & DCK & MGE1 & MGI & MGE2 & BGL1 & BGL2 & ABT1 & ABT2 \\
\hline \multirow{4}{*}{ Minor intervention } & Minimum number achieved & 0 & 0 & 0 & 1 & 0 & 0 & 0 & 0 \\
\cline { 2 - 11 } & Maximum number achieved & 4 & 3 & 3 & 3 & 3 & 3 & 3 & 3 \\
\cline { 2 - 10 } & Average & 2.51 & 2.62 & 1.80 & 2.61 & 1.70 & 1.61 & 1.05 & 1.04 \\
\cline { 2 - 10 } & Standard deviation & 0.74 & 0.57 & 0.75 & 0.57 & 1.03 & 1.01 & 0.82 & 0.88 \\
\hline \multirow{5}{*}{ Major intervention } & Minimum number achieved & 0 & 0 & 1 & 0 & 0 & 0 & 0 & 0 \\
\cline { 2 - 10 } & Maximum number achieved & 2 & 1 & 1 & 1 & 2 & 2 & 0 & 0 \\
\cline { 2 - 10 } & Average & 0.40 & 0.03 & 1 & 0.03 & 1.45 & 1.42 & 0 & 0 \\
\cline { 2 - 10 } & Standard deviation & 0.54 & 0.16 & 0 & 0.16 & 0.62 & 0.60 & 0 & 0 \\
\hline \multirow{5}{*}{ Replacement } & Minimum number achieved & 0 & 0 & 0 & 0 & 0 & 0 & 0 & 0 \\
\cline { 2 - 10 } & Maximum number achieved & 1 & 0 & 0 & 0 & 2 & 1 & 0 & 0 \\
\cline { 2 - 10 } & Average & 0.01 & 0 & 0 & 0 & 0.27 & 0.27 & 0 & 0 \\
\cline { 2 - 10 } & Standard deviation & 0.10 & 0 & 0 & 0 & 0.46 & 0.45 & 0 & 0 \\
\hline
\end{tabular}

Table D.2: Statistics on the expected number of interventions on each bridge components - maintenance strategy: repair as soon as possible

\begin{tabular}{|c|c|c|c|c|c|c|c|c|c|}
\hline & & DCK & MGE1 & MGI & MGE2 & BGL1 & BGL2 & ABT 1 & ABT 2 \\
\hline \multirow{4}{*}{ As new condition } & $\begin{array}{c}\text { Minimum number } \\
\text { achieved }\end{array}$ & 14.53 & 17.41 & 26.85 & 20.13 & 13.87 & 10.48 & 41.53 & 31.21 \\
\hline & $\begin{array}{c}\text { Maximum number } \\
\text { achieved }\end{array}$ & 52.00 & 52.00 & 51.00 & 52.00 & 51.00 & 51.00 & 60.00 & 60.00 \\
\hline & Average & 39.70 & 42.07 & 41.87 & 42.29 & 37.34 & 37.41 & 54.38 & 54.38 \\
\hline & Standard deviation & 8.14 & 5.98 & 4.60 & 6.12 & 7.72 & 8.27 & 4.57 & 4.91 \\
\hline \multirow{4}{*}{ Good condition } & $\begin{array}{c}\text { Minimum number } \\
\text { achieved }\end{array}$ & 4.50 & 5.69 & 0 & 8 & 0 & 0 & 0 & 0 \\
\hline & $\begin{array}{c}\text { Maximum number } \\
\text { achieved }\end{array}$ & 35.82 & 41.89 & 21.05 & 38.50 & 21.84 & 21.08 & 18.36 & 28.79 \\
\hline & Average & 17.65 & 17.14 & 8.41 & 16.93 & 9.26 & 9.02 & 4.93 & 4.93 \\
\hline & Standard deviation & 6.80 & 5.62 & 4.06 & 5.92 & 5.18 & 5.36 & 4.29 & 4.71 \\
\hline \multirow{4}{*}{ Poor condition } & $\begin{array}{c}\text { Minimum number } \\
\text { achieved }\end{array}$ & 0 & 0 & 9 & 0 & 2.27 & 2.72 & 0 & 0 \\
\hline & $\begin{array}{c}\text { Maximum number } \\
\text { achieved }\end{array}$ & 19.93 & 5.42 & 9.00 & 4.91 & 25.66 & 23.76 & 0 & 0 \\
\hline & Average & 1.89 & 0.09 & 9.00 & 0.08 & 11.47 & 11.45 & 0 & 0 \\
\hline & Standard deviation & 2.82 & 0.62 & 0.00 & 0.55 & 3.93 & 3.90 & 0 & 0 \\
\hline \multirow{4}{*}{$\begin{array}{l}\text { Very poor } \\
\text { condition }\end{array}$} & $\begin{array}{c}\text { Minimum number } \\
\text { achieved }\end{array}$ & 0 & 0 & 0 & 0 & 0 & 0 & 0 & 0 \\
\hline & $\begin{array}{c}\text { Maximum number } \\
\text { achieved }\end{array}$ & 5.37 & 0 & 0 & 0 & 11.39 & 9.91 & 0 & 0 \\
\hline & Average & 0.04 & 0 & 0 & 0 & 1.23 & 1.41 & 0 & 0 \\
\hline & Standard deviation & 0.40 & 0 & 0 & 0 & 2.34 & 2.47 & 0 & 0 \\
\hline
\end{tabular}

Table D.3: Statistics on the duration (years) spending in each condition state of each bridge components - maintenance strategy: repair as soon as possible 


\section{Intervention option 2}

\begin{tabular}{|c|c|c|c|c|c|c|c|c|c|}
\hline & & DCK & MGE1 & MGI & MGE2 & BGL1 & BGL2 & ABT1 & ABT2 \\
\hline \multirow{4}{*}{ Minor intervention } & Minimum number achieved & 0 & 0 & 0 & 0 & 0 & 0 & 0 & 0 \\
\hline & Maximum number achieved & 0 & 0 & 0 & 0 & 0 & 0 & 0 & 0 \\
\hline & Average & 0 & 0 & 0 & 0 & 0 & 0 & 0 & 0 \\
\hline & Standard deviation & 0 & 0 & 0 & 0 & 0 & 0 & 0 & 0 \\
\hline \multirow{4}{*}{ Major intervention } & Minimum number achieved & 0 & 1 & 1 & 1 & 0 & 0 & 0 & 0 \\
\hline & Maximum number achieved & 2 & 2 & 2 & 2 & 3 & 4 & 2 & 1 \\
\hline & Average & 1.78 & 1.21 & 1.77 & 1.22 & 1.98 & 1.96 & 0.2 & 0.25 \\
\hline & Standard deviation & 0.45 & 0.40 & 0.43 & 0.42 & 0.64 & 0.57 & 0.41 & 0.43 \\
\hline \multirow{4}{*}{ Replacement } & Minimum number achieved & 0 & 0 & 0 & 0 & 0 & 0 & 0 & 0 \\
\hline & Maximum number achieved & 2 & 0 & 0 & 0 & 3 & 2 & 0 & 0 \\
\hline & Average & 0.10 & 0 & 0 & 0 & 0.73 & 0.72 & 0 & 0 \\
\hline & Standard deviation & 0.32 & 0 & 0 & 0 & 0.61 & 0.52 & 0 & 0 \\
\hline
\end{tabular}

Table D.4: Statistics on the expected number of interventions on each bridge components - maintenance strategy: repair as soon as possible

\begin{tabular}{|c|c|c|c|c|c|c|c|c|c|}
\hline & & DCK & MGE1 & MGI & MGE2 & BGL1 & BGL2 & ABT 1 & ABT 2 \\
\hline \multirow{4}{*}{ As new condition } & $\begin{array}{c}\text { Minimum number } \\
\text { achieved }\end{array}$ & 2.89 & 0.85 & 6.27 & 0.99 & 3.50 & 6.69 & 0.55 & 0.37 \\
\hline & $\begin{array}{c}\text { Maximum number } \\
\text { achieved }\end{array}$ & 51.00 & 45.00 & 51.00 & 38.69 & 51.00 & 51.00 & 60.00 & 60.00 \\
\hline & Average & 26.03 & 18.16 & 26.11 & 17.63 & 28.85 & 27.81 & 36.22 & 34.44 \\
\hline & Standard deviation & 10.10 & 8.37 & 8.61 & 7.97 & 12.10 & 11.14 & 20.29 & 20.32 \\
\hline \multirow{4}{*}{ Good condition } & $\begin{array}{c}\text { Minimum number } \\
\text { achieved }\end{array}$ & 3.56 & 7.68 & 0 & 13.39 & 0 & 0 & 0 & 0 \\
\hline & $\begin{array}{c}\text { Maximum number } \\
\text { achieved }\end{array}$ & 43.36 & 55.08 & 36.65 & 53.08 & 19.68 & 16.72 & 58.28 & 58.55 \\
\hline & Average & 21.14 & 34.32 & 19.93 & 34.48 & 7.44 & 7.76 & 22.02 & 23.46 \\
\hline & Standard deviation & 6.90 & 8.08 & 7.69 & 7.79 & 4.18 & 3.84 & 19.58 & 19.04 \\
\hline \multirow{4}{*}{ Poor condition } & $\begin{array}{c}\text { Minimum number } \\
\text { achieved }\end{array}$ & 2.57 & 3.07 & 9 & 3.08 & 4.19 & 4.12 & 0 & 0 \\
\hline & $\begin{array}{c}\text { Maximum number } \\
\text { achieved }\end{array}$ & 26.79 & 14.14 & 17.96 & 14.55 & 31.09 & 35.83 & 8.98 & 8.90 \\
\hline & Average & 11.60 & 6.78 & 13.21 & 7.11 & 18.63 & 19.54 & 1.02 & 1.38 \\
\hline & Standard deviation & 4.44 & 2.47 & 2.90 & 2.36 & 6.21 & 5.99 & 2.31 & 2.58 \\
\hline \multirow{4}{*}{$\begin{array}{l}\text { Very poor } \\
\text { condition }\end{array}$} & $\begin{array}{c}\text { Minimum number } \\
\text { achieved }\end{array}$ & 0 & 0 & 0 & 0 & 0 & 0 & 0 & 0 \\
\hline & $\begin{array}{c}\text { Maximum number } \\
\text { achieved }\end{array}$ & 7.42 & 0 & 0 & 0 & 14.32 & 13.12 & 0 & 0 \\
\hline & Average & 0.45 & 0 & 0 & 0 & 4.29 & 4.12 & 0 & 0 \\
\hline & Standard deviation & 1.45 & 0 & 0 & 0 & 3.75 & 3.33 & 0 & 0 \\
\hline
\end{tabular}

Table D.5: Statistics on the duration (years) spending in each condition state of each bridge components - maintenance strategy: repair as soon as possible 


\section{Intervention option 3}

\begin{tabular}{|c|c|c|c|c|c|c|c|c|c|}
\hline & & DCK & MGE1 & MGI & MGE2 & BGL1 & BGL2 & ABT1 & ABT2 \\
\hline \multirow{4}{*}{ Minor intervention } & Minimum number achieved & 0 & 0 & 0 & 0 & 0 & 0 & 0 & 0 \\
\hline & Maximum number achieved & 3 & 3 & 2 & 3 & 3 & 5 & 3 & 3 \\
\hline & Average & 2 & 3 & 0 & 3 & 1 & 1 & 1 & 1 \\
\hline & Standard deviation & 1 & 1 & 0 & 1 & 1 & 1 & 1 & 1 \\
\hline \multirow{4}{*}{ Major intervention } & Minimum number achieved & 0 & 0 & 0 & 0 & 0 & 0 & 0 & 0 \\
\hline & Maximum number achieved & 0 & 0 & 0 & 0 & 0 & 0 & 0 & 0 \\
\hline & Average & 0 & 0 & 0 & 0 & 0 & 0 & 0 & 0 \\
\hline & Standard deviation & 0 & 0 & 0 & 0 & 0 & 0 & 0 & 0 \\
\hline \multirow{4}{*}{ Replacement } & Minimum number achieved & 0 & 0 & 0 & 0 & 1 & 1 & 0 & 0 \\
\hline & Maximum number achieved & 2 & 1 & 1 & 0 & 3 & 3 & 0 & 0 \\
\hline & Average & 0.23 & 0.01 & 0.08 & 0 & 1.50 & 1.48 & 0 & 0 \\
\hline & Standard deviation & 0.44 & 0.07 & 0.27 & 0 & 0.61 & 0.57 & 0 & 0 \\
\hline
\end{tabular}

Table D.6: Statistics on the expected number of interventions on each bridge components - maintenance strategy: repair as soon as possible

\begin{tabular}{|c|c|c|c|c|c|c|c|c|c|}
\hline & & DCK & MGE1 & MGI & MGE2 & BGL1 & BGL2 & ABT 1 & ABT 2 \\
\hline \multirow{4}{*}{ As new condition } & $\begin{array}{c}\text { Minimum number } \\
\text { achieved }\end{array}$ & 7.45 & 0 & 0 & 0 & 6.09 & 6.87 & 19.74 & 14.57 \\
\hline & $\begin{array}{c}\text { Maximum number } \\
\text { achieved }\end{array}$ & 52.00 & 52.00 & 32.95 & 52.00 & 47.71 & 50.00 & 60.00 & 60.00 \\
\hline & Average & 36.80 & 42.06 & 0.61 & 42.07 & 29.96 & 29.68 & 53.40 & 53.94 \\
\hline & Standard deviation & 9.90 & 6.15 & 3.28 & 6.34 & 9.13 & 9.70 & 6.27 & 6.30 \\
\hline \multirow{4}{*}{ Good condition } & $\begin{array}{c}\text { Minimum number } \\
\text { achieved }\end{array}$ & 3.13 & 1.61 & 0 & 3.75 & 0 & 0 & 0 & 0 \\
\hline & $\begin{array}{c}\text { Maximum number } \\
\text { achieved }\end{array}$ & 35.80 & 34.70 & 9.62 & 34.19 & 19.32 & 21.64 & 40.26 & 33.41 \\
\hline & Average & 17.03 & 16.59 & 0.12 & 16.50 & 6.93 & 7.00 & 5.57 & 4.82 \\
\hline & Standard deviation & 6.66 & 5.09 & 0.85 & 4.76 & 4.05 & 4.44 & 6.09 & 5.29 \\
\hline \multirow{4}{*}{ Poor condition } & $\begin{array}{c}\text { Minimum number } \\
\text { achieved }\end{array}$ & 0.00 & 0 & 16.03 & 0 & 2.54 & 2.53 & 0 & 0 \\
\hline & $\begin{array}{c}\text { Maximum number } \\
\text { achieved }\end{array}$ & 25.00 & 57.40 & 60.00 & 56.25 & 29.18 & 29.90 & 0.00 & 42.73 \\
\hline & Average & 3.56 & 0.29 & 57.77 & 0.39 & 12.18 & 12.31 & 0.00 & 0.21 \\
\hline & Standard deviation & 6.23 & 4.06 & 5.86 & 4.08 & 5.28 & 5.09 & 0.00 & 3.02 \\
\hline \multirow{4}{*}{$\begin{array}{l}\text { Very poor } \\
\text { condition }\end{array}$} & $\begin{array}{c}\text { Minimum number } \\
\text { achieved }\end{array}$ & 0 & 0 & 0 & 0 & 4.13 & 4.00 & 0 & 0 \\
\hline & $\begin{array}{c}\text { Maximum number } \\
\text { achieved }\end{array}$ & 17.43 & 1.00 & 9.97 & 0 & 20.27 & 21.56 & 0 & 0 \\
\hline & Average & 1.57 & 0.00 & 0.48 & 0 & 9.88 & 9.97 & 0 & 0 \\
\hline & Standard deviation & 3.28 & 0.07 & 1.73 & 0 & 4.05 & 4.14 & 0 & 0 \\
\hline
\end{tabular}

Table D.7: Statistics on the duration (years) spending in each condition state of each bridge components - maintenance strategy: repair as soon as possible 


\section{Intervention option 4}

\begin{tabular}{|c|c|c|c|c|c|c|c|c|c|}
\hline & & DCK & MGE1 & MGI & MGE2 & BGL1 & BGL2 & ABT1 & ABT2 \\
\hline \multirow{4}{*}{ Minor intervention } & Minimum number achieved & 0 & 0 & 0 & 0 & 0 & 0 & 0 & 0 \\
\hline & Maximum number achieved & 0 & 0 & 0 & 0 & 0 & 0 & 0 & 0 \\
\hline & Average & 0 & 0 & 0 & 0 & 0 & 0 & 0 & 0 \\
\hline & Standard deviation & 0 & 0 & 0 & 0 & 0 & 0 & 0 & 0 \\
\hline \multirow{4}{*}{ Major intervention } & Minimum number achieved & 0 & 0 & 0 & 0 & 0 & 0 & 0 & 0 \\
\hline & Maximum number achieved & 0 & 0 & 0 & 0 & 0 & 0 & 0 & 0 \\
\hline & Average & 0 & 0 & 0 & 0 & 0 & 0 & 0 & 0 \\
\hline & Standard deviation & 0 & 0 & 0 & 0 & 0 & 0 & 0 & 0 \\
\hline \multirow{4}{*}{ Replacement } & Minimum number achieved & 1 & 0 & 0 & 0 & 1 & 1 & 0 & 0 \\
\hline & Maximum number achieved & 2 & 1 & 1 & 1 & 4 & 3 & 0 & 0 \\
\hline & Average & 1.29 & 0.03 & 0.11 & 0.02 & 2.18 & 2.14 & 0 & 0 \\
\hline & Standard deviation & 0.45 & 0.17 & 0.31 & 0.12 & 0.65 & 0.62 & 0 & 0 \\
\hline
\end{tabular}

Table D.8: Statistics on the expected number of interventions on each bridge components - maintenance strategy: repair as soon as possible

\begin{tabular}{|c|c|c|c|c|c|c|c|c|c|}
\hline & & DCK & MGE1 & MGI & MGE2 & BGL1 & BGL2 & ABT 1 & ABT 2 \\
\hline \multirow{4}{*}{ As new condition } & $\begin{array}{c}\text { Minimum number } \\
\text { achieved }\end{array}$ & 0.02 & 0 & 0 & 0 & 1.77 & 1.48 & 0.06 & 0.16 \\
\hline & $\begin{array}{c}\text { Maximum number } \\
\text { achieved }\end{array}$ & 48.00 & 8.68 & 12.37 & 0 & 45.76 & 50.00 & 60.00 & 60.00 \\
\hline & Average & 15.87 & 0.12 & 0.35 & 0 & 20.58 & 20.71 & 34.71 & 34.10 \\
\hline & Standard deviation & 9.89 & 0.91 & 1.57 & 0 & 10.47 & 10.67 & 21.03 & 21.17 \\
\hline \multirow{4}{*}{ Good condition } & $\begin{array}{c}\text { Minimum number } \\
\text { achieved }\end{array}$ & 2.25 & 5.27 & 0 & 4.16 & 0 & 0 & 0 & 0 \\
\hline & $\begin{array}{c}\text { Maximum number } \\
\text { achieved }\end{array}$ & 34.61 & 46.70 & 15.35 & 51.86 & 13.51 & 14.86 & 59.05 & 59.51 \\
\hline & Average & 17.18 & 24.09 & 0.20 & 24.42 & 5.70 & 5.96 & 21.16 & 22.43 \\
\hline & Standard deviation & 5.98 & 8.03 & 1.50 & 8.84 & 3.10 & 3.24 & 18.57 & 19.34 \\
\hline \multirow{4}{*}{ Poor condition } & $\begin{array}{c}\text { Minimum number } \\
\text { achieved }\end{array}$ & 3.53 & 8.79 & 24.55 & 6.14 & 2.96 & 3.44 & 0 & 0 \\
\hline & $\begin{array}{c}\text { Maximum number } \\
\text { achieved }\end{array}$ & 35.60 & 52.82 & 60.00 & 55.84 & 31.45 & 30.62 & 38.34 & 33.61 \\
\hline & Average & 17.02 & 34.11 & 57.27 & 34.01 & 17.85 & 17.39 & 2.61 & 1.95 \\
\hline & Standard deviation & 7.32 & 8.50 & 5.17 & 9.11 & 5.43 & 5.37 & 6.27 & 5.62 \\
\hline \multirow{4}{*}{$\begin{array}{l}\text { Very poor } \\
\text { condition }\end{array}$} & $\begin{array}{c}\text { Minimum number } \\
\text { achieved }\end{array}$ & 4.04 & 0 & 0 & 0 & 4.37 & 4.05 & 0 & 0 \\
\hline & $\begin{array}{c}\text { Maximum number } \\
\text { achieved }\end{array}$ & 16.85 & 7.55 & 9.70 & 3.58 & 25.62 & 23.70 & 0 & 0 \\
\hline & Average & 8.40 & 0.16 & 0.66 & 0.05 & 14.33 & 14.39 & 0 & 0 \\
\hline & Standard deviation & 3.03 & 1.02 & 2.13 & 0.39 & 4.22 & 4.21 & 0 & 0 \\
\hline
\end{tabular}

Table D.9: Statistics on the duration (years) spending in each condition state of each bridge components - maintenance strategy: repair as soon as possible 